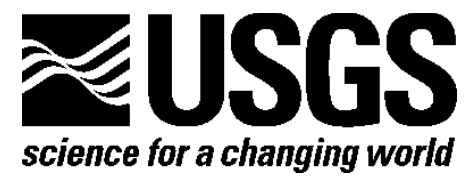

\title{
USGS Compilation of Geographic Information System (GIS) Data Representing Coal Mines and Coal-Bearing Areas in China
}

Compiled by Michael H. Trippi, Harvey E. Belkin, Shifeng Dai, Susan J. Tewalt, and Chiu-Jung Chou

Open-File Report 2014-1219

U.S. Department of the Interior

U.S. Geological Survey 


\section{U.S. Department of the Interior \\ SALLY JEWELL, Secretary}

\section{U.S. Geological Survey \\ Suzette M. Kimball, Acting Director}

U.S. Geological Survey, Reston, Virginia: 2014

For more information on the USGS_-the Federal source for science about the Earth,

its natural and living resources, natural hazards, and the environment-visit

http://www.usgs.gov or call 1-888-ASK-USGS (1-888-275-8747)

For an overview of USGS information products, including maps, imagery, and publications, visit $h$ ttp://www.usgs.gov/pubprod

To order this and other USGS information products, visit http://store.usgs.gov

Any use of trade, firm, or product names is for descriptive purposes only and does not imply endorsement by the U.S. Government.

Although this information product, for the most part, is in the public domain, it also may contain copyrighted materials as noted in the text. Permission to reproduce copyrighted items must be secured from the copyright owner.

Suggested citation:

Trippi, M.H., Belkin, H.E., Dai, Shifeng, Tewalt, S.J., and Chou, C.J., 2014, comps., USGS compilation of geographic information system (GIS) data representing coal mines and coal-bearing areas in China: U.S. Geological Survey Open-File Report 2014-1219, 135 p., http://dx.doi.org/10.3133/ofr20141219.

ISSN 2331-1258 (online) 


\section{Acknowledgments}

Major-, minor-, and trace-element data were provided by the USGS Energy Resources Program Geochemistry Laboratory, and ultimate and proximate analyses by Geochemical Testing, Inc. of

Somerset, Pa. We express appreciation for the instructive reviews by Christopher Garrity of the U.S. Geological Survey, and also by Dr. Yuzhuang Sun of the Department of Geology at Hebei University of Engineering in Handan, China. 


\section{Contents}

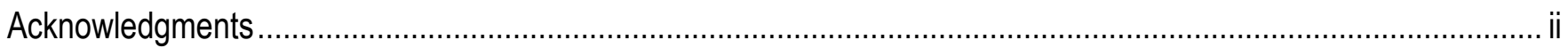

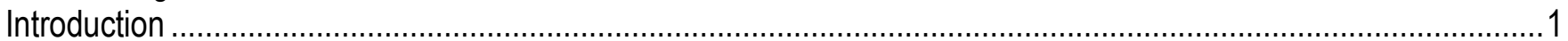

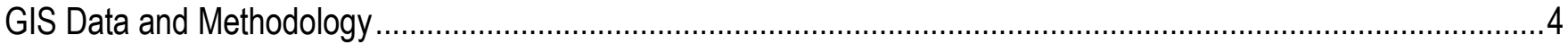

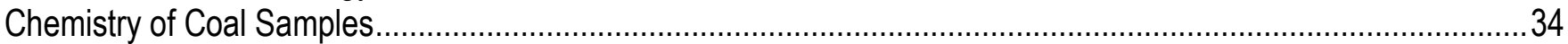

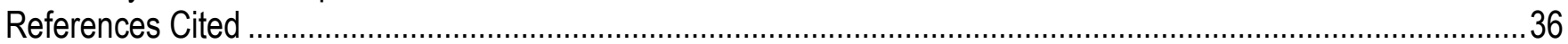

\section{Figures}

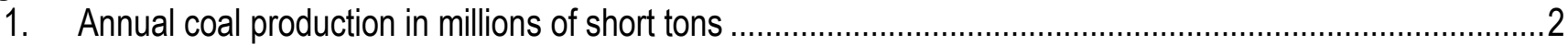

2. Provinces, municipalities, autonomous regions, and special administrative regions of China........................ 3

3. Coal mines and coal-mining areas of Anhui Province ......................................................................

4. Coal mines and coal-mining areas of Beijing Municipality, Hebei Province, and Tianjin Municipality. ...........6

5. Coal mines and coal-mining areas of Fujian Province ........................................................................

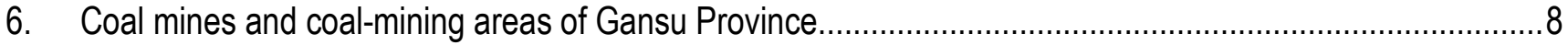

7. Coal mines and coal-mining areas of Guangdong Province .............................................................

8. Coal mines and coal-mining areas of Guangxi Zhuang Autonomous Region ..........................................10

9. Coal mines and coal-mining areas of Guizhou Province ....................................................................11

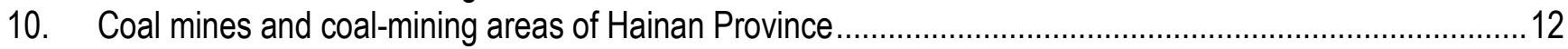

11. Coal mines and coal-mining areas of Heilongjiang Province ................................................................. 13

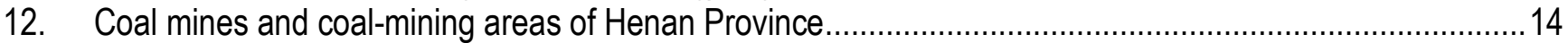

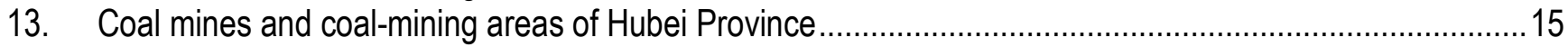

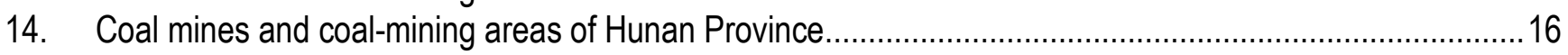

15. Coal mines and coal-mining areas of Inner Mongolia Autonomous Region ................................................ 17

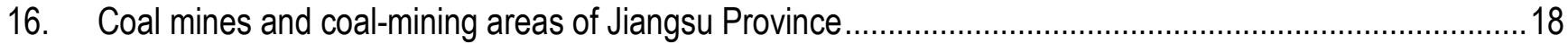

17. Coal mines and coal-mining areas of Jiangxi Province ...................................................................... 19

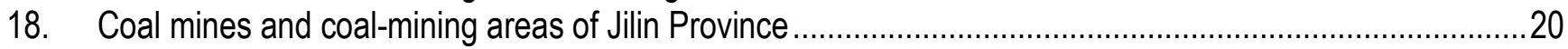

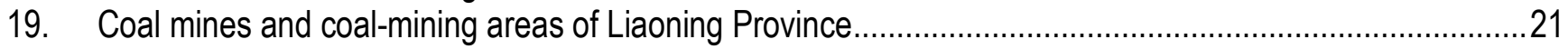

20. Coal mines and coal-mining areas of Ninxia Hui Autonomous Region ..................................................22

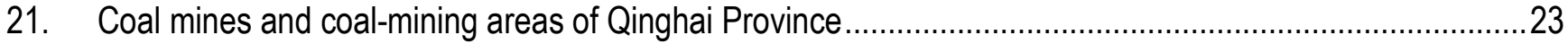

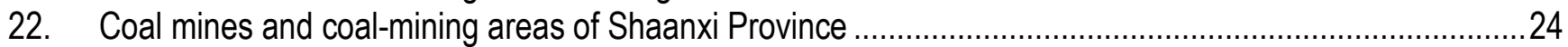

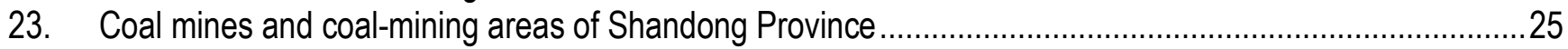

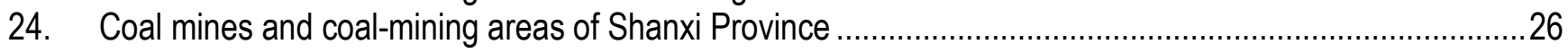

25. Coal mines and coal-mining areas of Sichuan Province and Chongqing Municipality ................................2

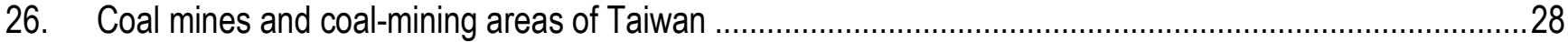

27. Coal mines and coal-mining areas of Xinjiang Uyghur Autonomous Region.............................................29

28. Coal mines and coal-mining areas of Xizang (Tibet) Autonomous Region ..............................................30

29. Coal mines and coal-mining areas of Yunnan Province .......................................................................

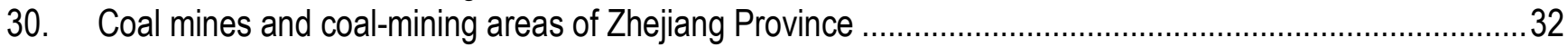

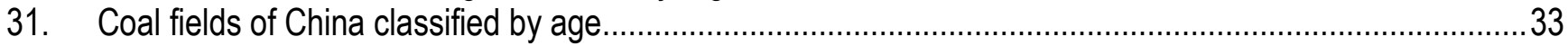

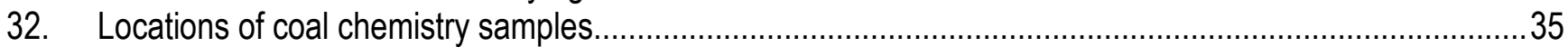

\section{Tables}

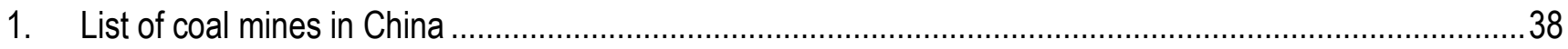




\section{Conversion Factors}

\begin{tabular}{|c|c|c|}
\hline Multiply & By & To obtain \\
\hline \multicolumn{3}{|c|}{ Length } \\
\hline meter $(\mathrm{m})$ & 3.281 & feet (ft) \\
\hline kilometer (km) & 0.6215 & mile (mi) \\
\hline meter (m) & 1.094 & yard (yd) \\
\hline \multicolumn{3}{|c|}{ Mass } \\
\hline tonne (t) (Metric ton) (2205 pounds) & 1 & Megagram (Mg) \\
\hline tonne (t) (Metric ton) (2205 pounds) & 0.9070 & ton, short ( 2,000 pounds) \\
\hline tonne ( $\mathrm{t}$ (Metric ton) (2205 pounds) & 1.016 & ton, long $(2,240 \mathrm{lb})$ \\
\hline
\end{tabular}




\title{
USGS Compilation of Geographic Information System (GIS) Data Representing the Coal Mines and Coal-Bearing Areas of China
}

\author{
Compiled by Michael H. Trippi, ${ }^{1}$ Harvey E. Belkin, ${ }^{1}$ Shifeng Dai, ${ }^{2}$ Susan J. Tewalt, ${ }^{3}$ and Chiu-Jung Chou ${ }^{4}$
}

\section{Introduction}

China depends on coal for most of its energy supply and surpassed the United States in coal production in 1983 (fig. 1). In 2009, China consumed about 3.5 billion short tons (3.175 tonnes) of coal, over 46 percent of the world total, which accounted for 71 percent of China's total primary energy consumption (Energy Information Administration, 2011). By 2010, China's coal consumption increased to 48.2 percent of the world's coal production, and accounted for nearly two-thirds of global consumption growth (British Petroleum, 2011). According to the World Energy Council (2010), in 2009 China had the third largest amount of recoverable coal reserves at 114.5 billion short tons (103.87 tonnes) (about 14 percent of the world's total reserves), surpassed only by the United States and Russia (Energy Information Administration, 2011). Most Chinese coal resources are bituminous in rank and range from early Carboniferous to Neogene in age (Dai and others, 2012). Shanxi province has been a major supplier of coal resources in the past, and Inner Mongolia has become very important in recent years (Energy Information Administration, 2011). Xinjiang Uyghur Autonomous Region, with up to 2.2 trillion tonnes of reserves (including the newly discovered 89.2 billion tonne Sha'er Lake reserve) may become the largest producer of Chinese coal in the near future (World Coal Association, 2011; China Daily, 2011). Figure 2 shows province, municipality, and autonomous region locations.

\footnotetext{
${ }^{1}$ U.S. Geological Survey.

${ }^{2}$ State Key Laboratory of Coal Resources and Safe Mining, Beijing, China.

${ }^{3}$ Retired, U.S. Geological Survey.

${ }^{4}$ Volunteer, U.S. Geological Survey.
} 


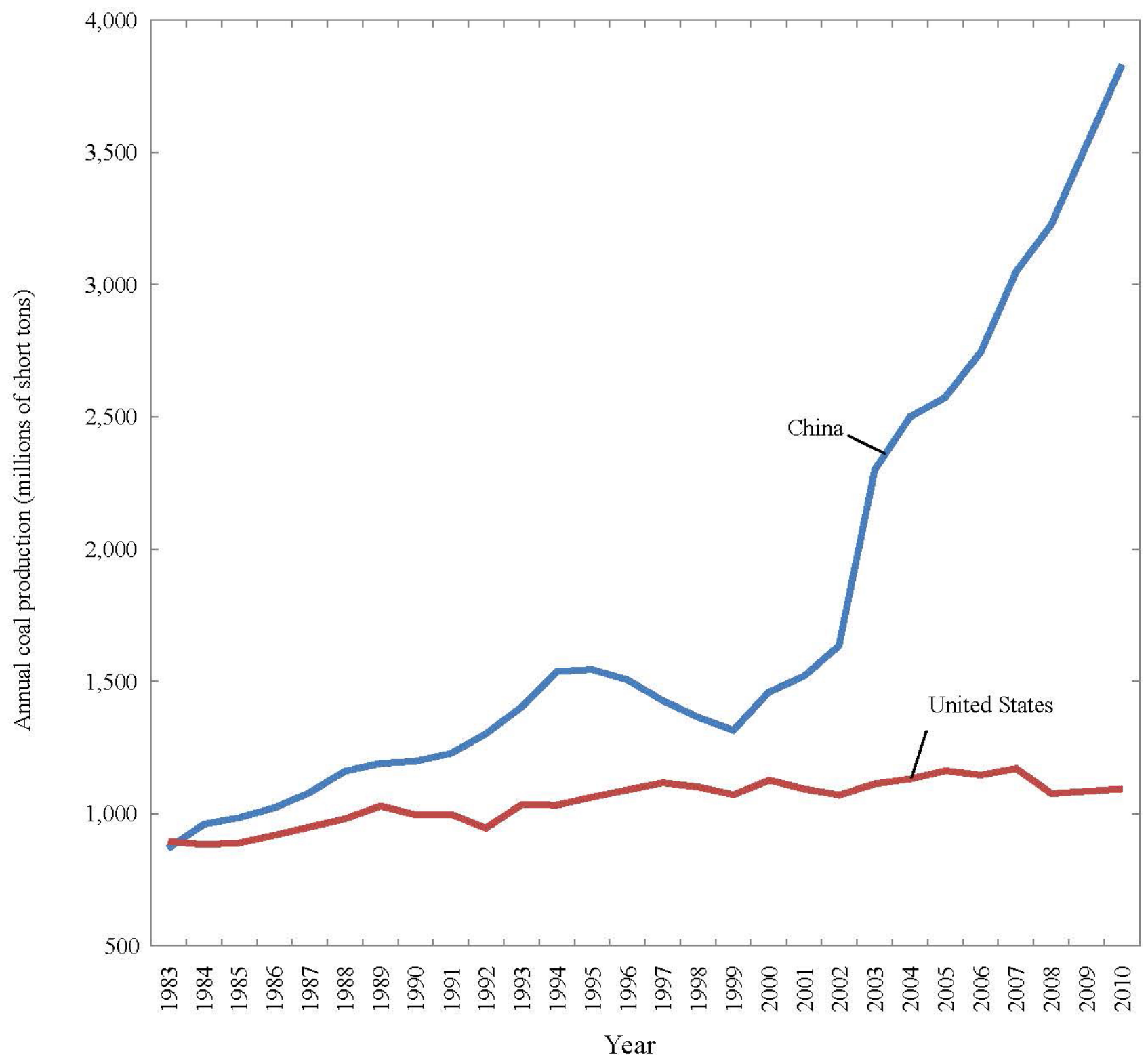

Figure 1. Annual coal production in millions of short tons (data source: Energy Information Administration, 2012). 


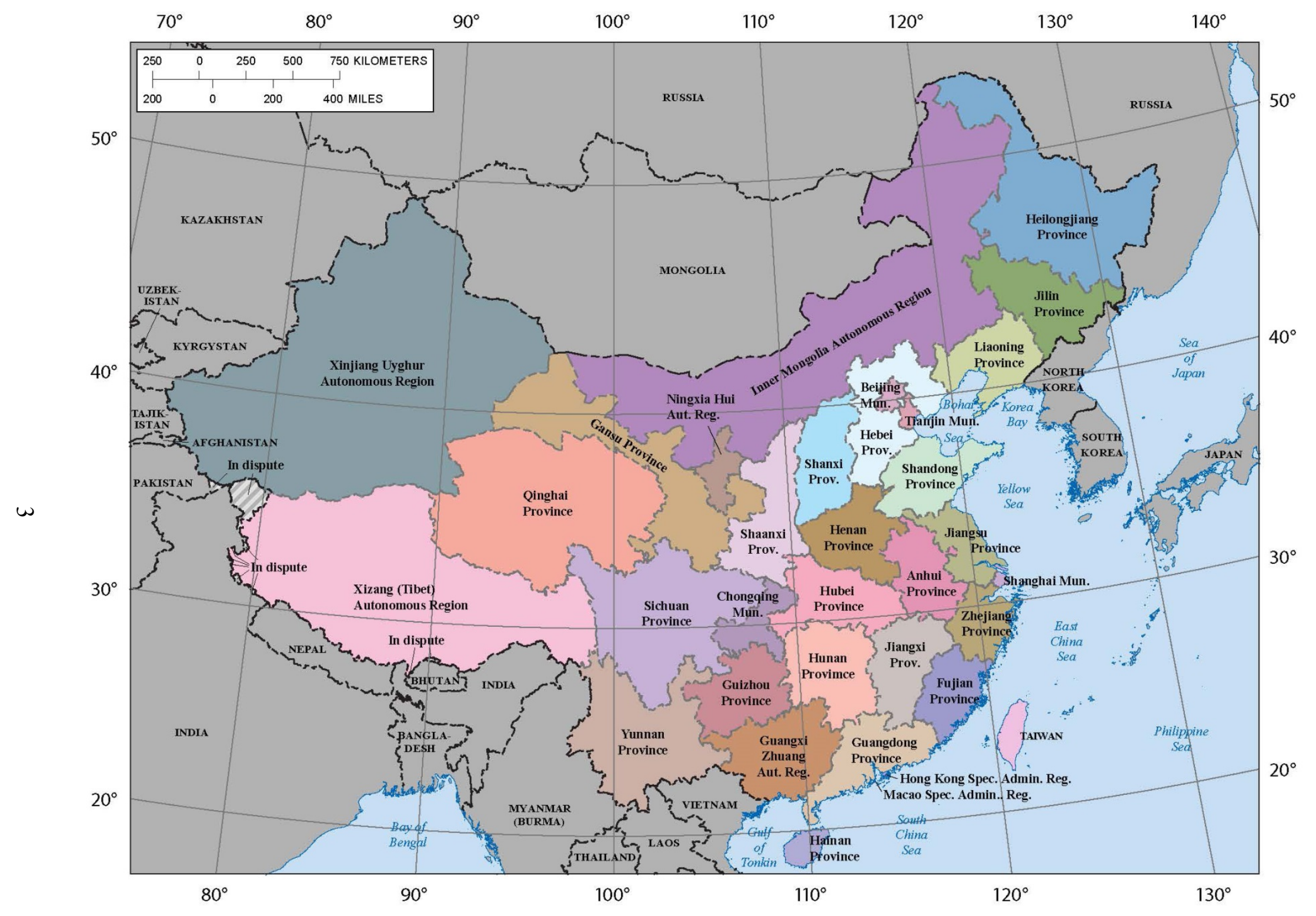

Figure 2. Provinces, municipalities, autonomous regions, and special administrative regions of China. Abbreviations used: Aut. Reg. Autonomous Region; Mun. - Municipality; Prov. - Province; Spec. Admin. Reg. - Special Administrative Region. 
This report is not intended to be an authoritative or comprehensive study of the coals that are available for mining and combustion in China. Publications of the Chinese Academy of Geological Sciences and the Bureau of Geological Survey of the Ministry of Geology and Mineral Resources, as well as many recent research journal articles, are available for more detailed information.

\section{GIS Data and Methodology}

The U.S. Geological Survey (USGS) has compiled geographic information system (GIS) data representing the known coal mine locations and coal-mining areas of China as of 2001. These data are now available for download, and may be used in a GIS for a variety of energy resource and environmental studies of China. These files were made by digitizing hardcopy maps created by the Chinese Academy of Geological Sciences and the Bureau of Geological Survey of the Ministry of Geology and Mineral Resources and published in the "Atlas of Solid Fuel and Nonmetal Resources of China" (Wang, 2001). Maps showing point locations of coal mines were scanned and georeferenced to (1) known latitude and longitude line intersections based on latitude and longitude reference marks from the original map or (2) to recognizable deviations in state and national boundary lines and shorelines. Base map projection information for the original paper maps was unknown, so the scanned images were adjusted to fit a base map with the following parameters: Projection, Lambert Conformal Conic; False Easting, 500,000 meters; False Northing, 500,000 meters; Central Meridian, $100^{\circ}$ W.; Standard Parallel 1, $18^{\circ}$ N.; Standard Parallel 2, $24^{\circ}$ N.; Latitude of Origin, $21^{\circ}$ N.; Linear Unit, Meter; Datum, World Geodetic System 1972 Beijing. Point locations of coal mines were digitized from the original maps and labeled with numbers that match rows in table 1, which lists coal mines and the type of coal being mined, by province. Regions of coal-mining and coal-bearing strata were created by creating buffers with 10-kilometer radii around each mine location, and joining overlapping circles. These regions were color-coded to indicate the type of coal (anthracite, bituminous, or lignite) being mined at each location. Detailed processing steps are described in the associated metadata files. Province-scale maps were also created to display the point locations of coal mines and the coal-mining areas described above (figs. 3-30). 


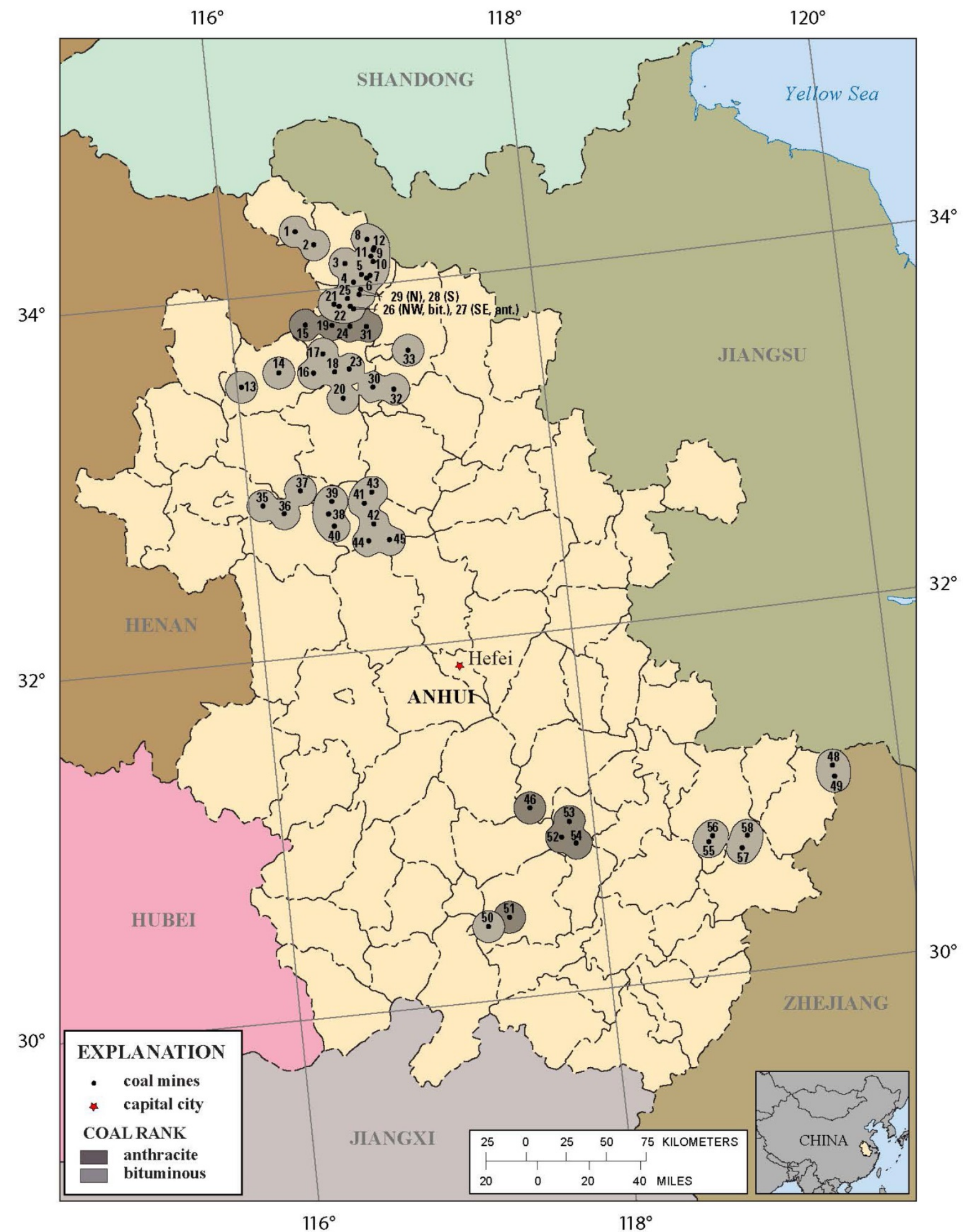

Figure 3. Coal mines and coal-mining areas of Anhui Province. Numbers are ID numbers in table 1. Abbreviations used: ant. - anthracite; bit. - bituminous; N - North; NW - Northwest; S - South; SE - Southeast. Coalmining areas are within 10-kilometer buffers of mine locations. 


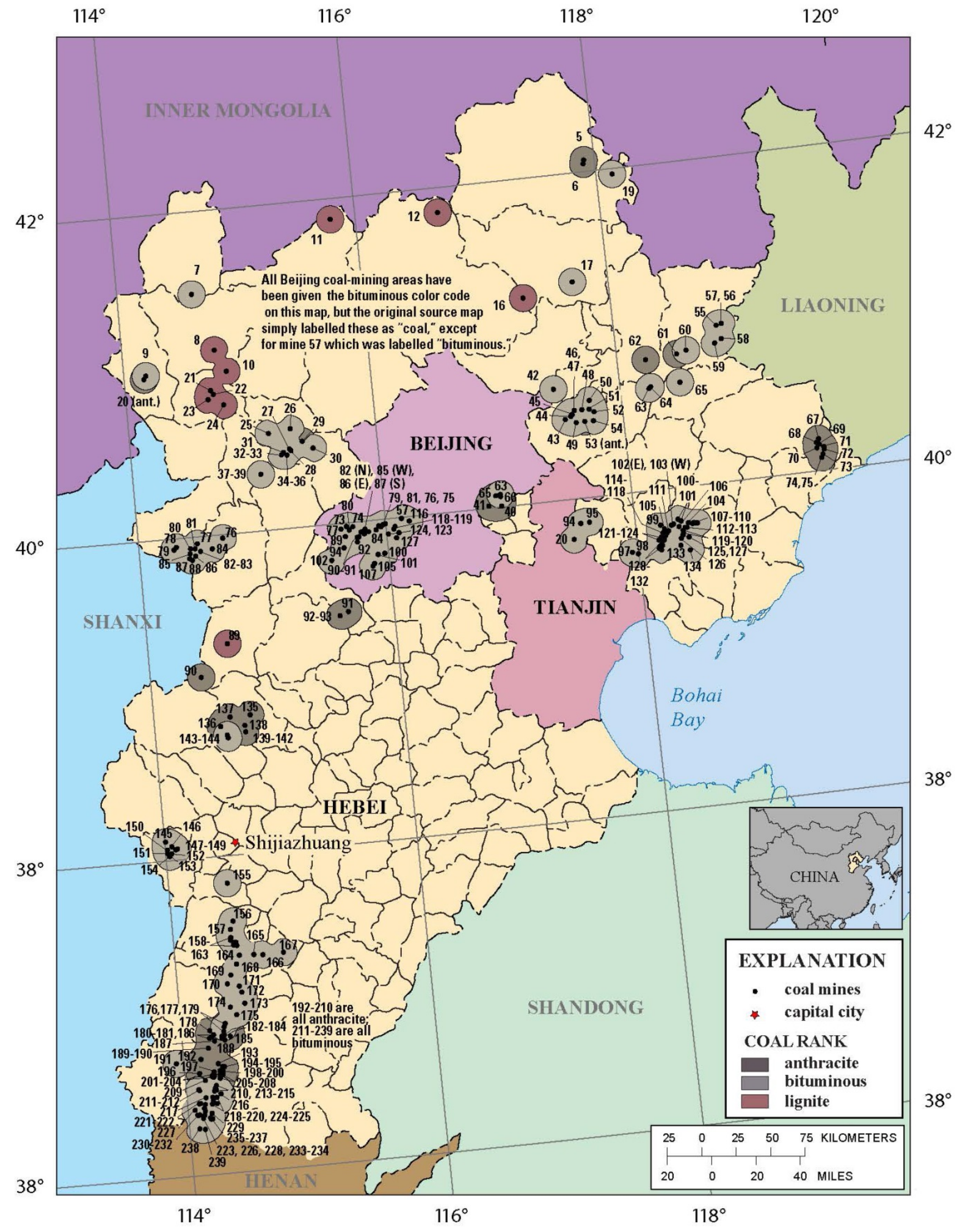

Figure 4. Coal mines and coal-mining areas of Beijing Municipality, Hebei Province, and Tianjin Municipality. Numbers are ID numbers in table 1. Abbreviations used: ant. - anthracite; E - East; N - North; S - South; W - West. Coal-mining areas are within 10-kilometer buffers of mine locations. 


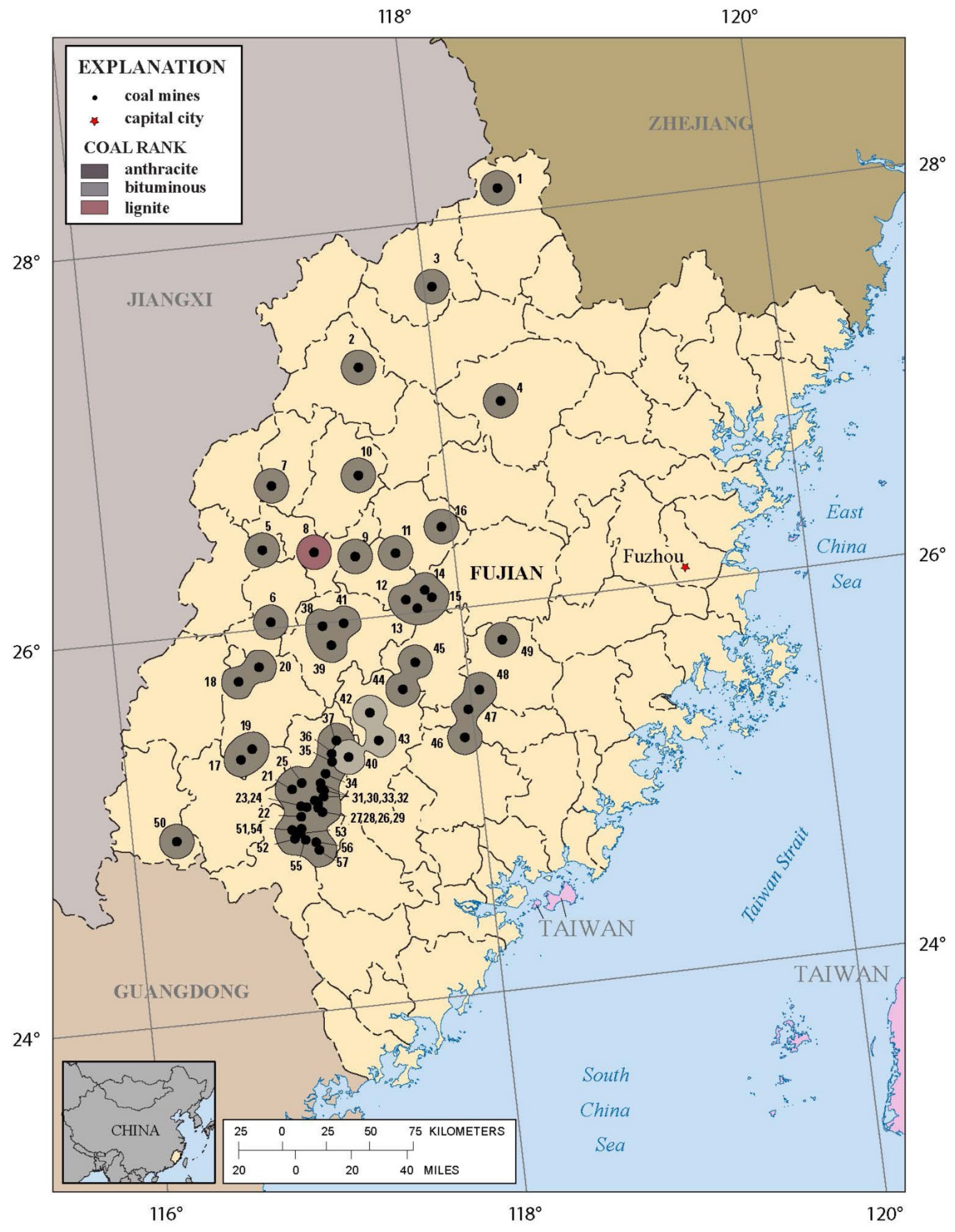

Figure 5. Coal mines and coal-mining areas of Fujian Province. Numbers are ID numbers in table 1. Coal-mining areas are within 10-kilometer buffers of mine locations. 


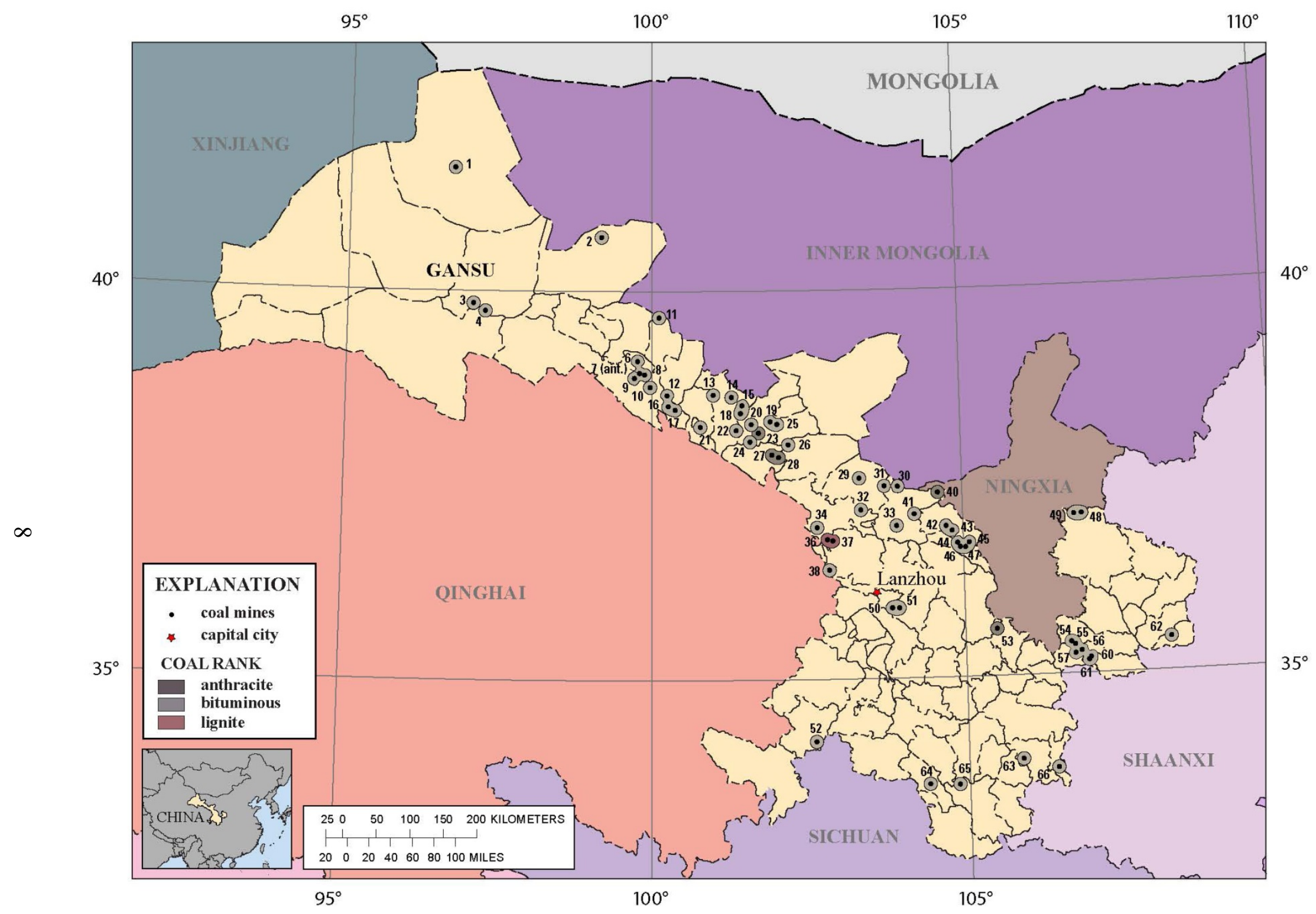

Figure 6. Coal mines and coal-mining areas of Gansu Province. Numbers are ID numbers in table 1. Abbreviation used: ant. - anthracite. Coalmining areas are within 10-kilometer buffers of mine locations. 


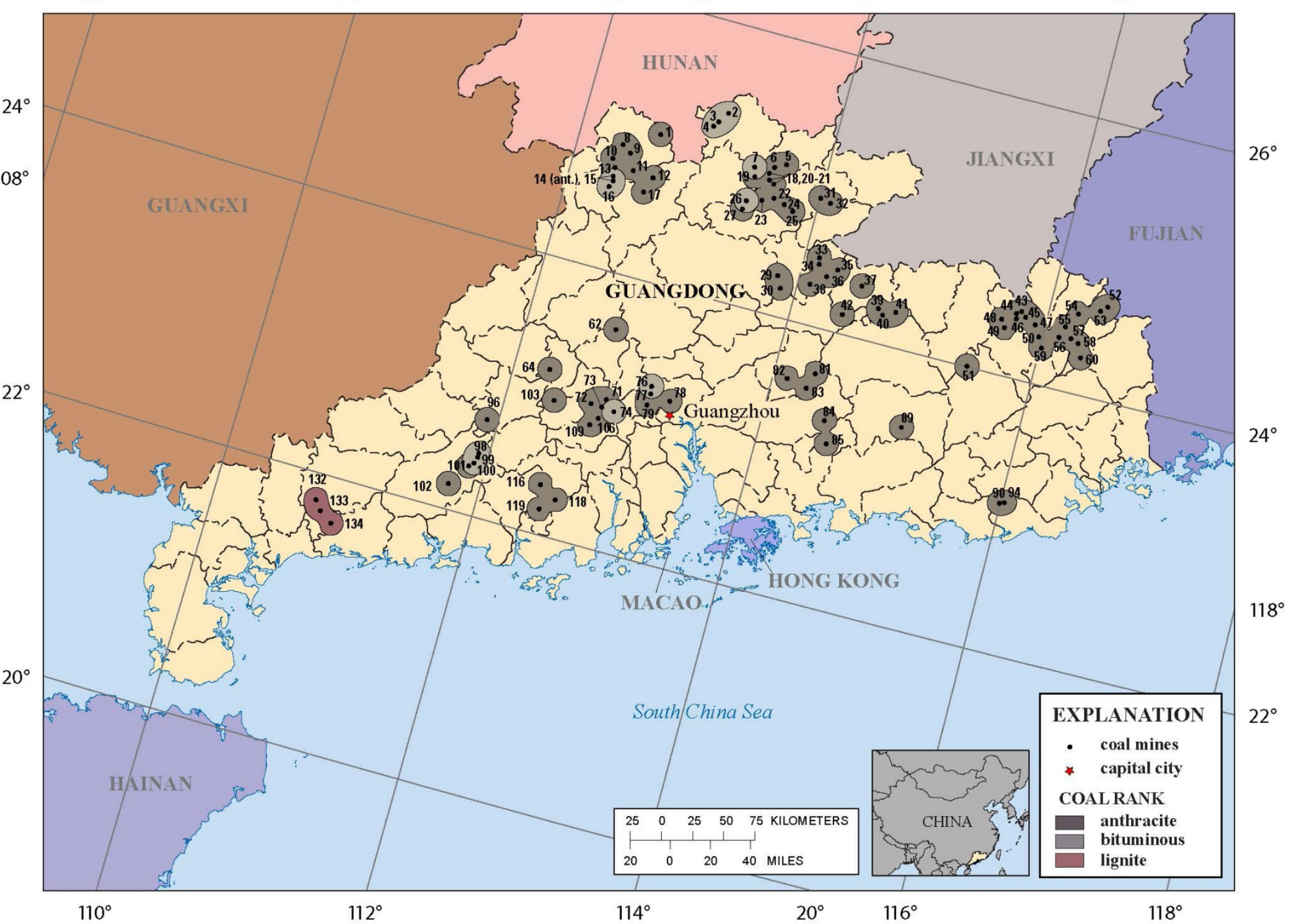

Figure 7. Coal mines and coal-mining areas of Guangdong Province. Numbers are ID numbers in table 1. Abbreviation used: ant. - anthracite. Coal-mining areas are within 10-kilometer buffers of mine locations. 
$26^{\circ}$

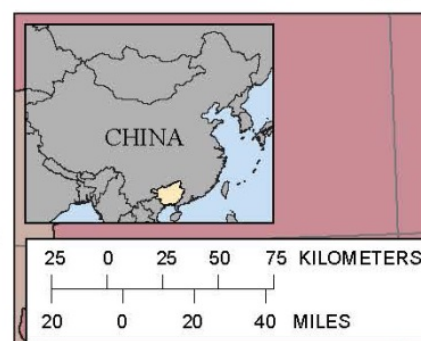

$24^{\circ}$

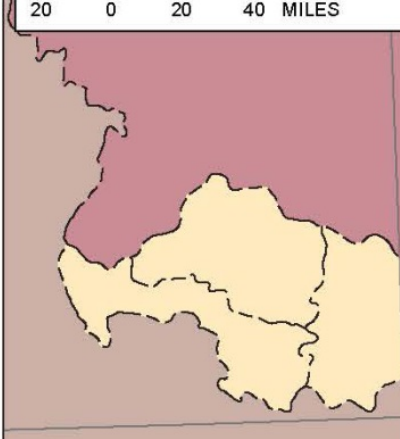

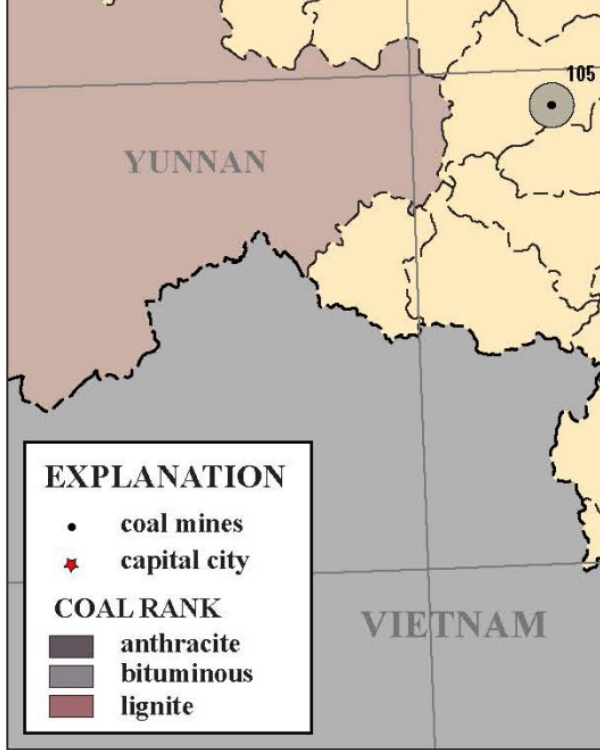

$106^{\circ}$

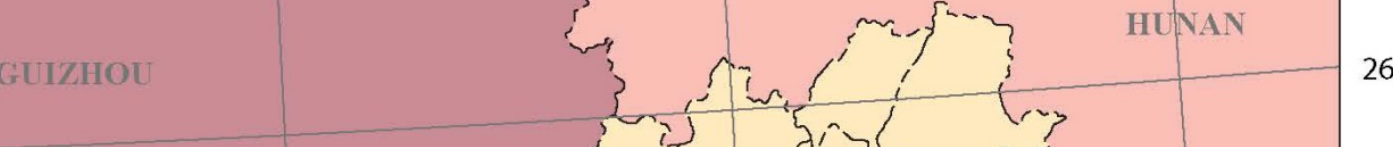

Figure 8. Coal mines and coal-mining areas of Guangxi Zhuang Autonomous Region. Numbers are ID numbers in table 1. Coal-mining areas are within 10-kilometer buffers of mine locations. 


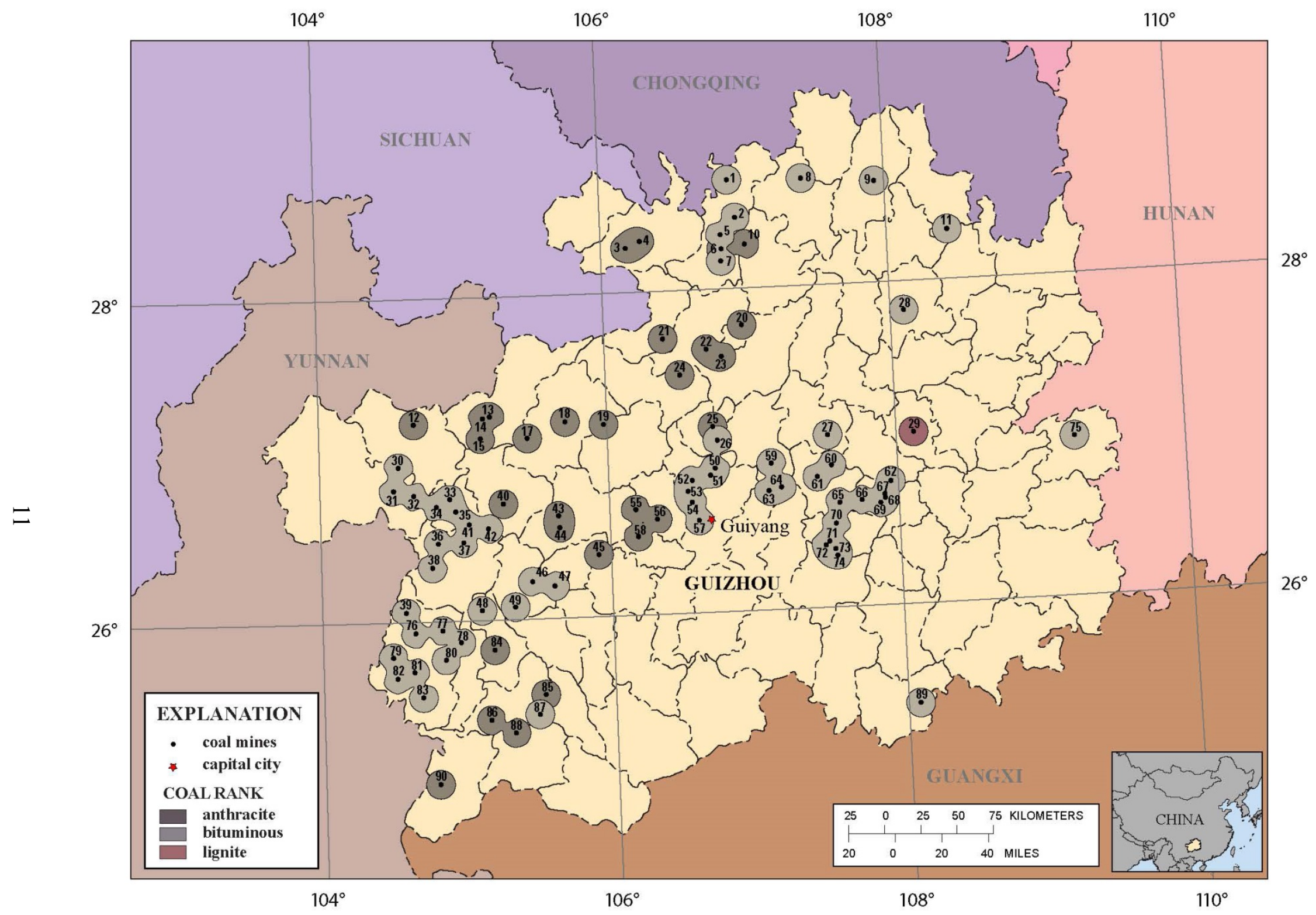

Figure 9. Coal mines and coal-mining areas of Guizhou Province. Numbers are ID numbers in table 1. Coal-mining areas are within 10-kilometer buffers of mine locations. 


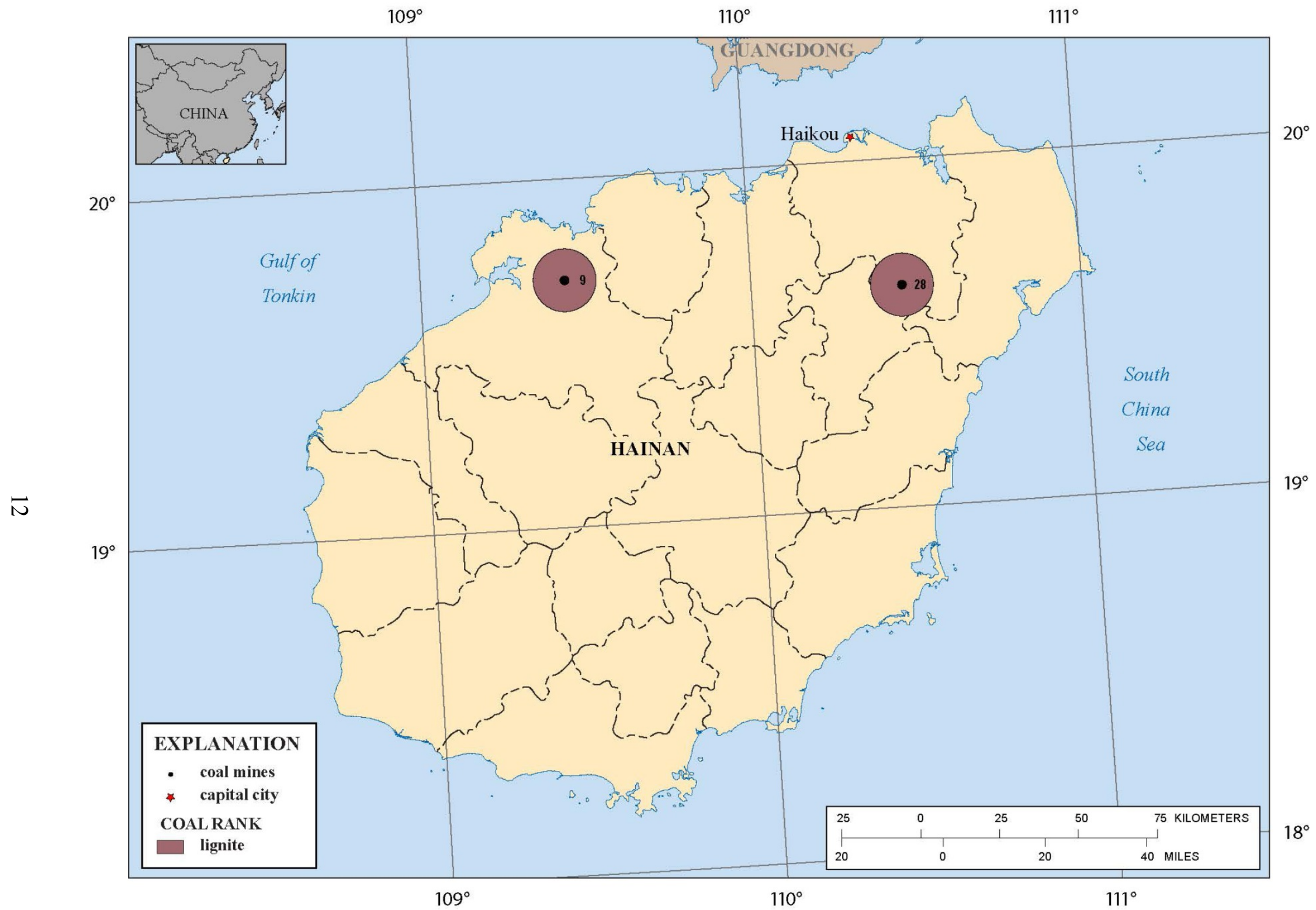

Figure 10. Coal mines and coal-mining areas of Hainan Province. Numbers are ID numbers in table 1. Coal-mining areas are within 10-kilometer buffers of mine locations. 


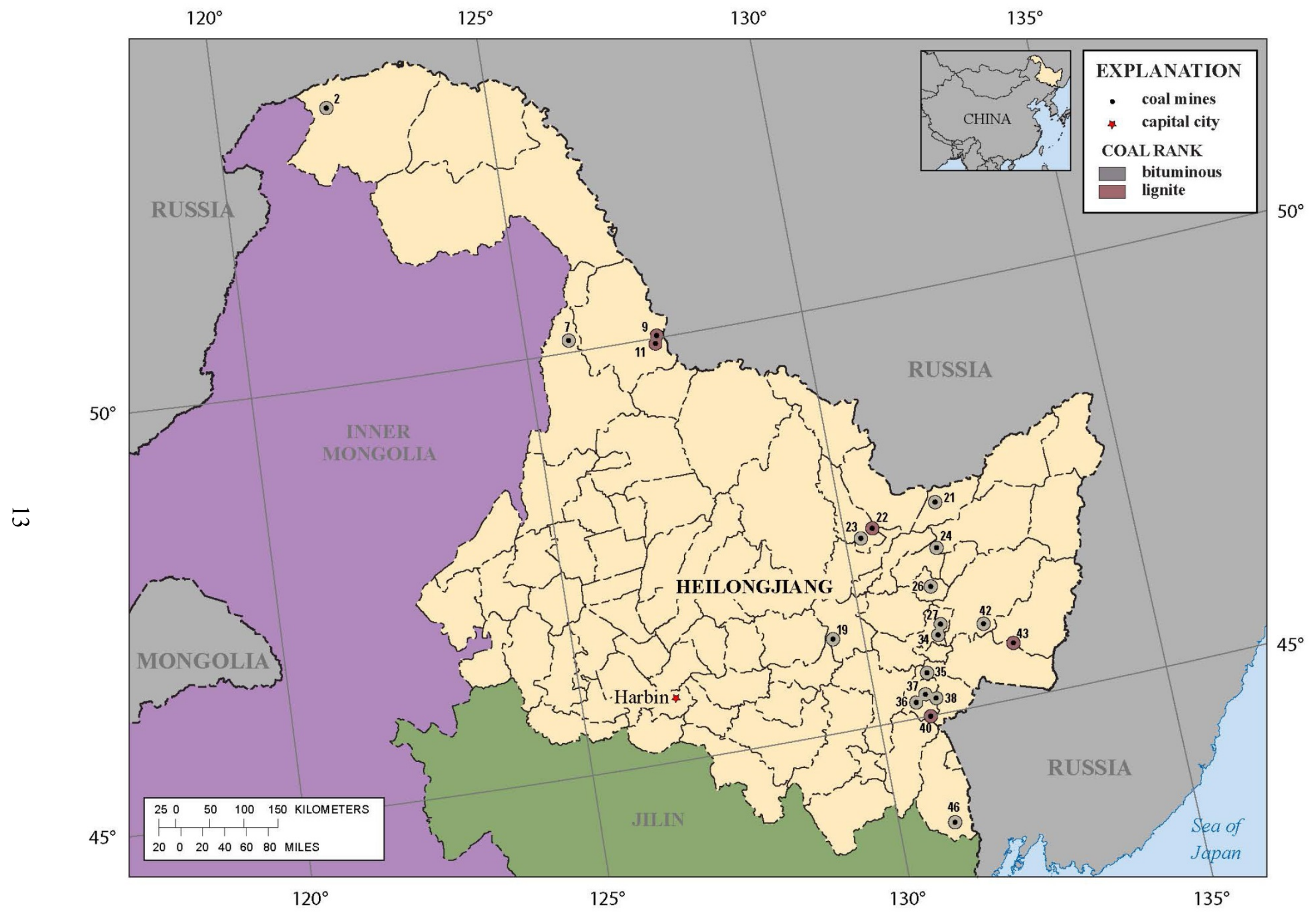

Figure 11. Coal mines and coal-mining areas of Heilongjiang Province. Numbers are ID numbers in table 1. Coal-mining areas are within 10kilometer buffers of mine locations. 


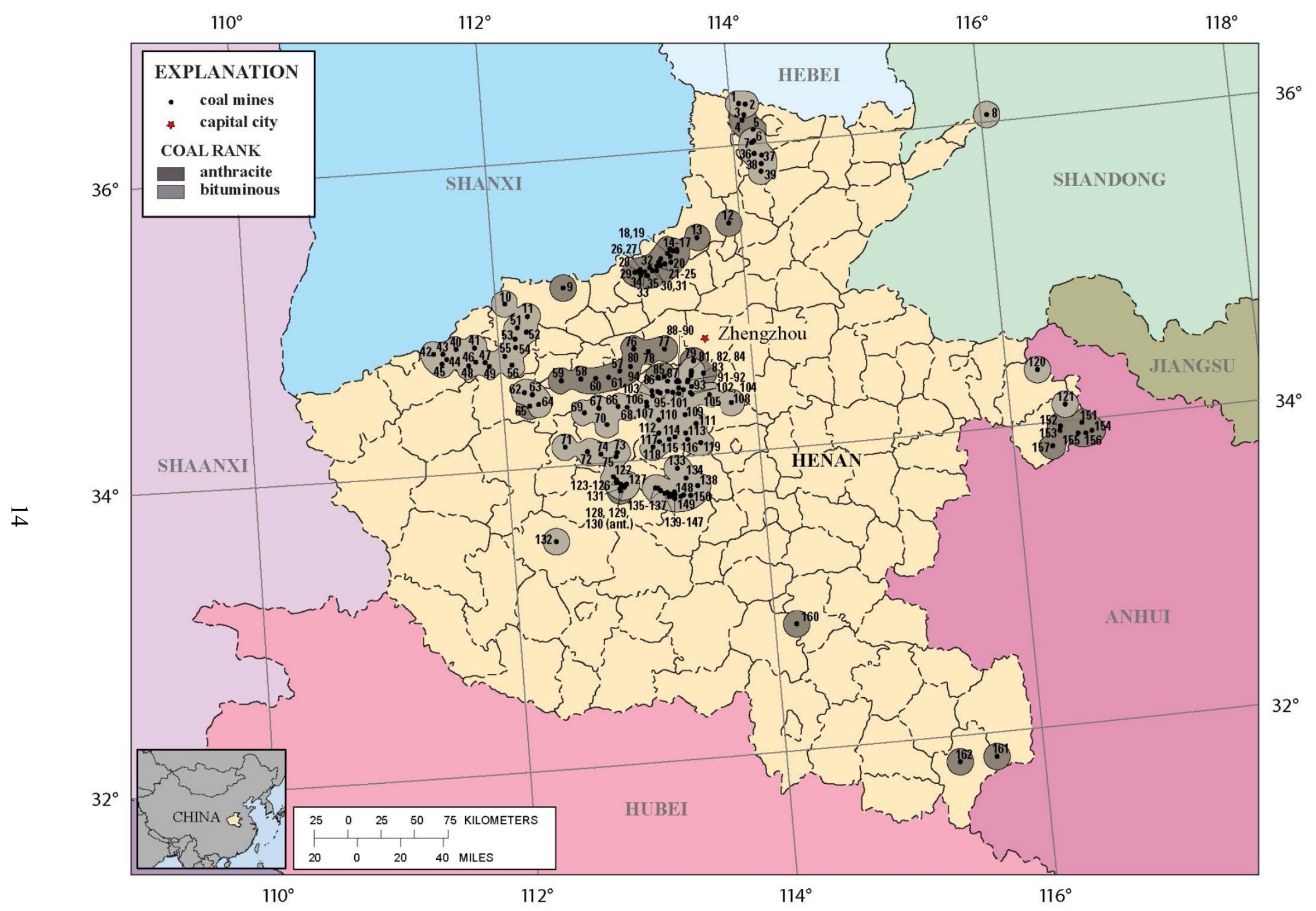

Figure 12. Coal mines and coal-mining areas of Henan Province. Numbers are ID numbers in table 1. Abbreviation used: ant. - anthracite. Coalmining areas are within 10-kilometer buffers of mine locations. 


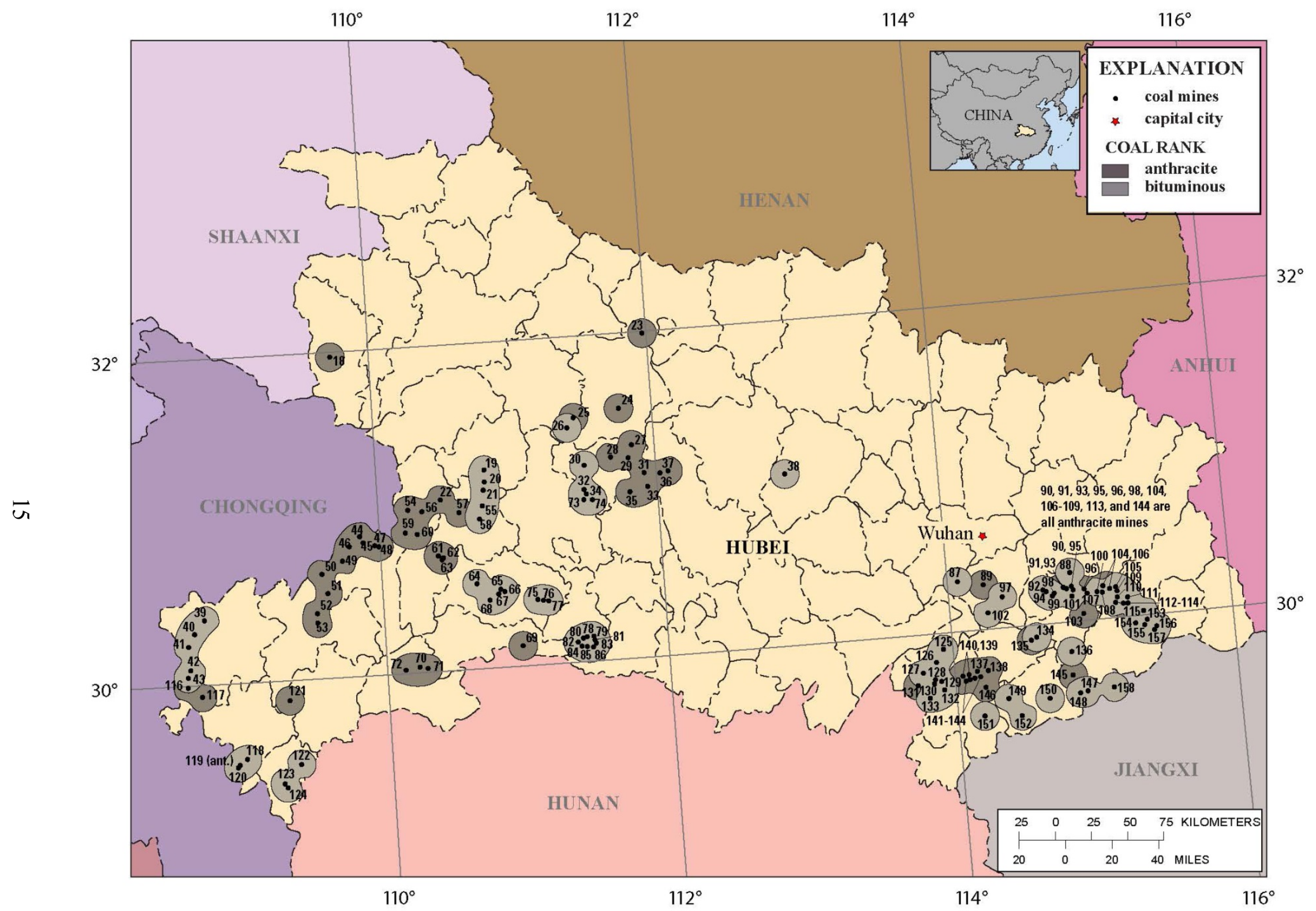

Figure 13. Coal mines and coal-mining areas of Hubei Province. Numbers are ID numbers in table 1. Abbreviation used: ant. - anthracite. Coalmining areas are within 10-kilometer buffers of mine locations. 


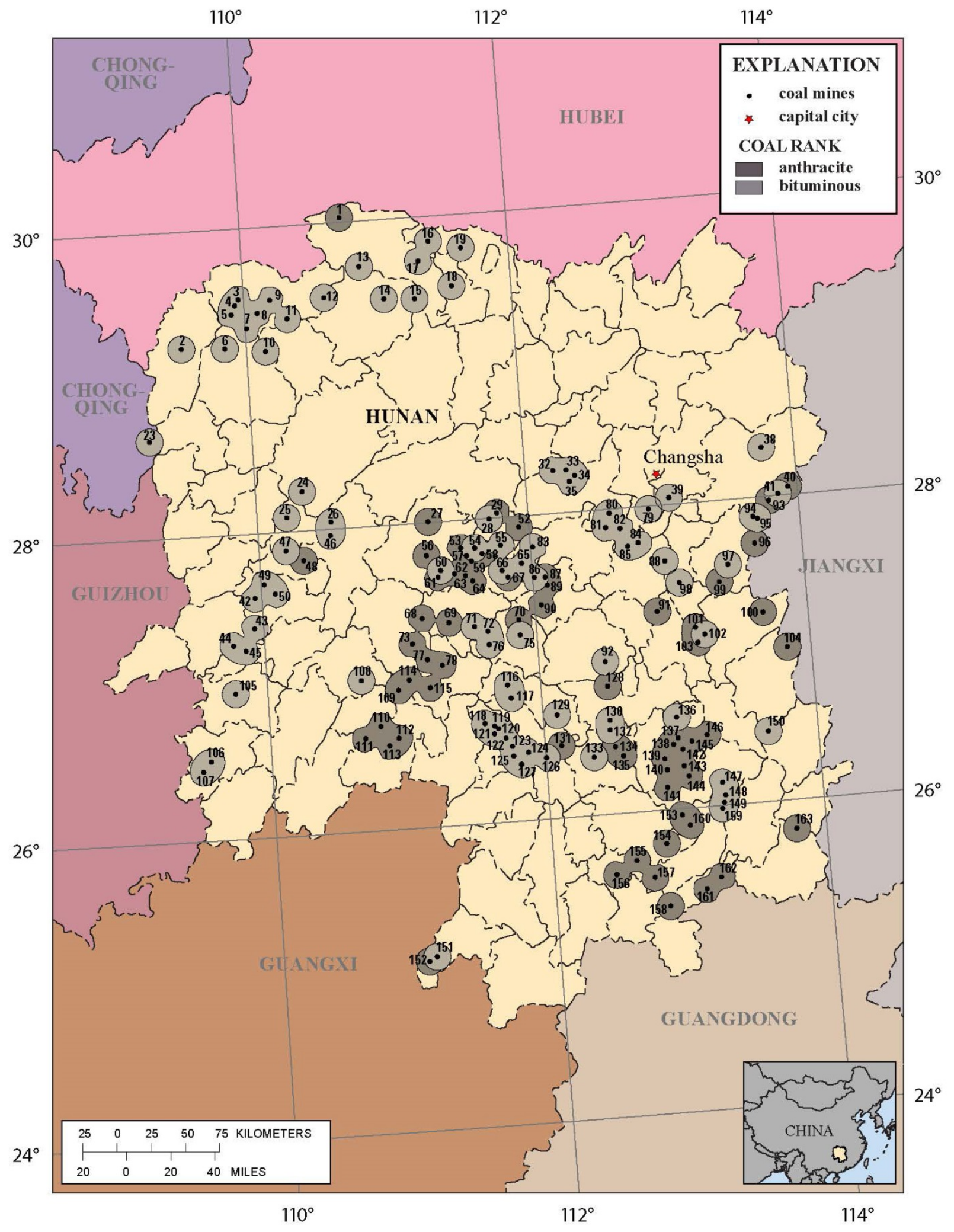

Figure 14. Coal mines and coal-mining areas of Hunan Province. Numbers are ID numbers in table 1. Coalmining areas are within 10-kilometer buffers of mine locations. 


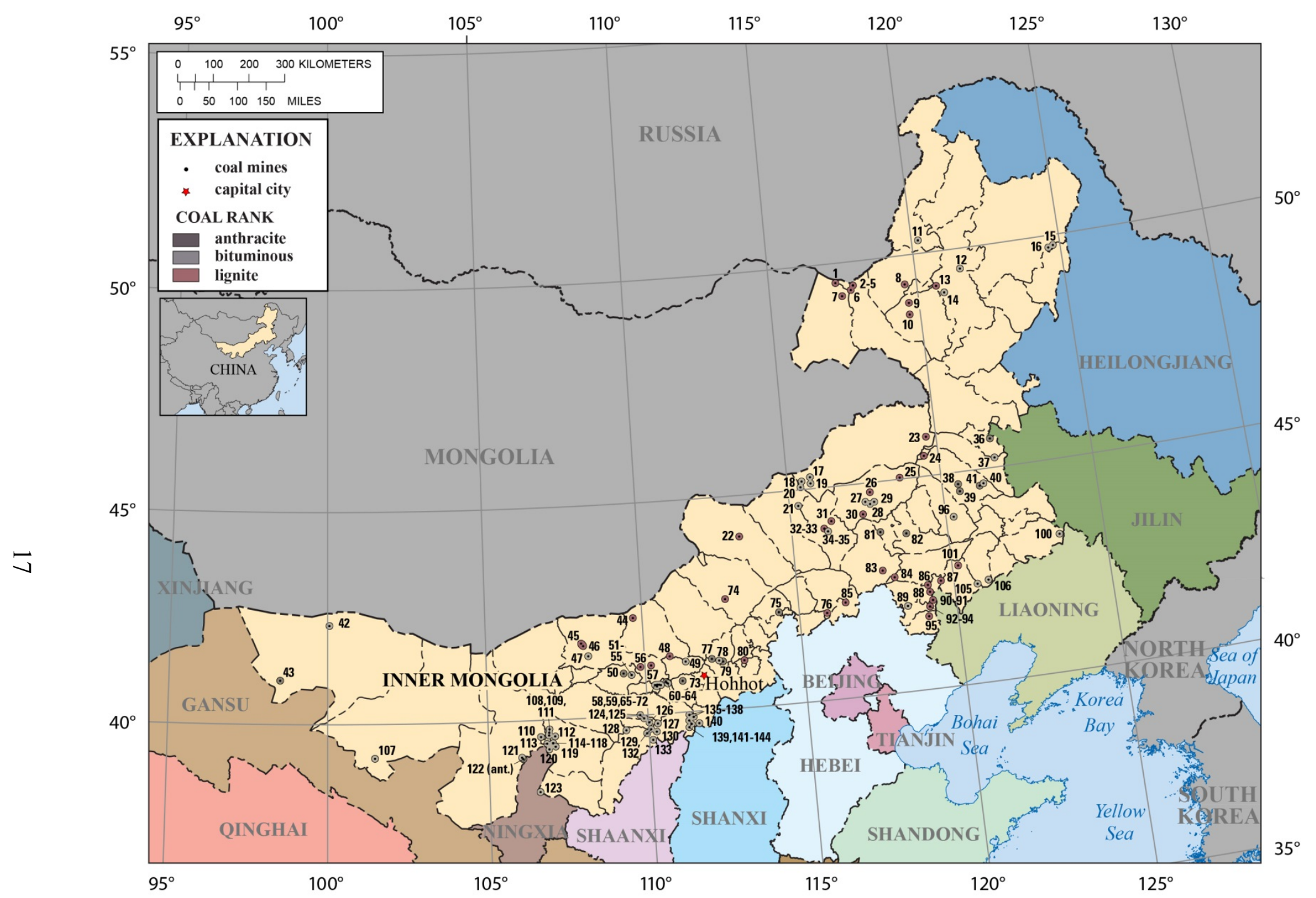

Figure 15. Coal mines and coal-mining areas of Inner Mongolia Autonomous Region. Numbers are ID numbers in table 1. Abbreviation used: ant. anthracite. Coal-mining areas are within 10-kilometer buffers of mine locations. 


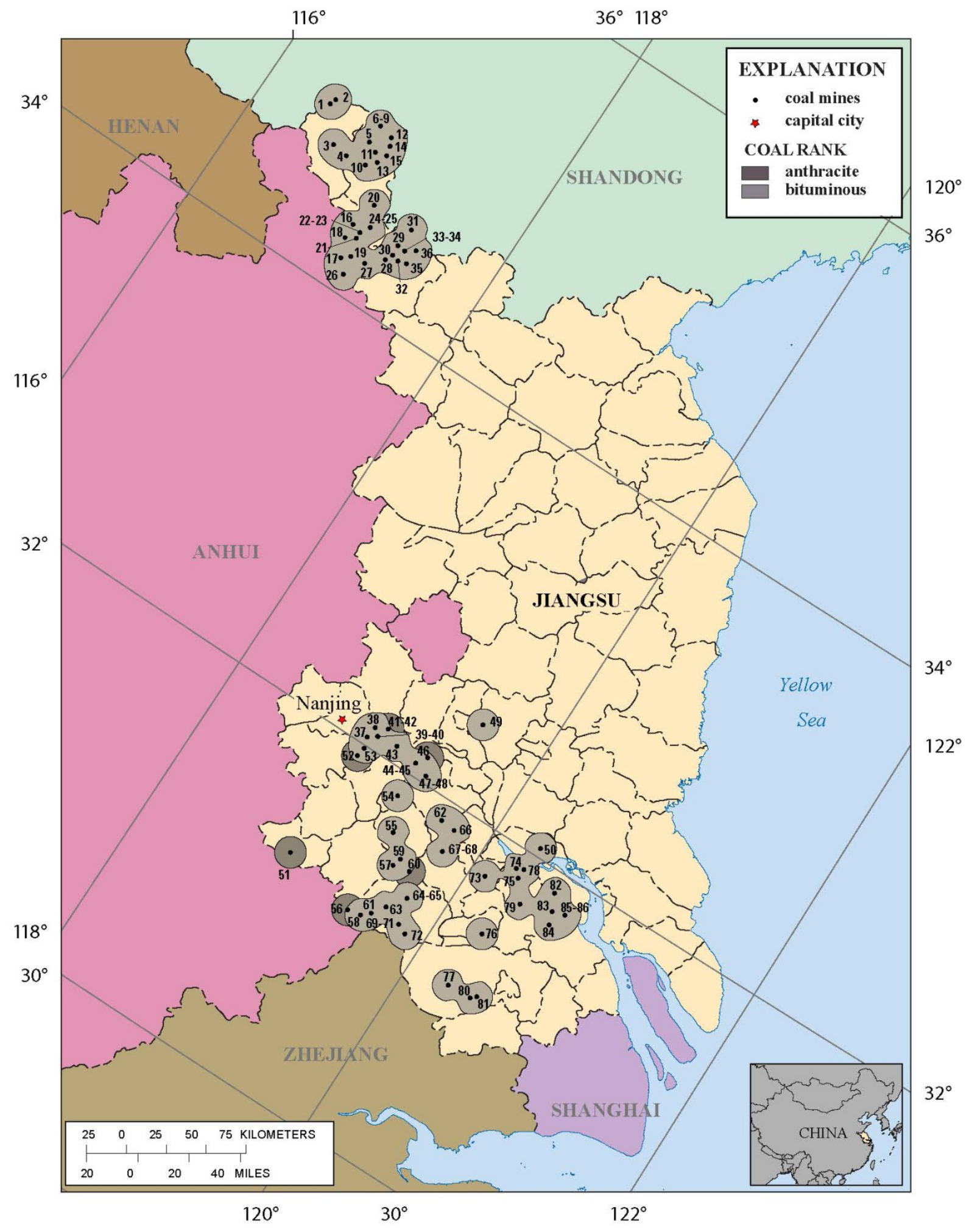

Figure 16. Coal mines and coal-mining areas of Jiangsu Province. Numbers are ID numbers in table 1. Coalmining areas are within 10-kilometer buffers of mine locations. 


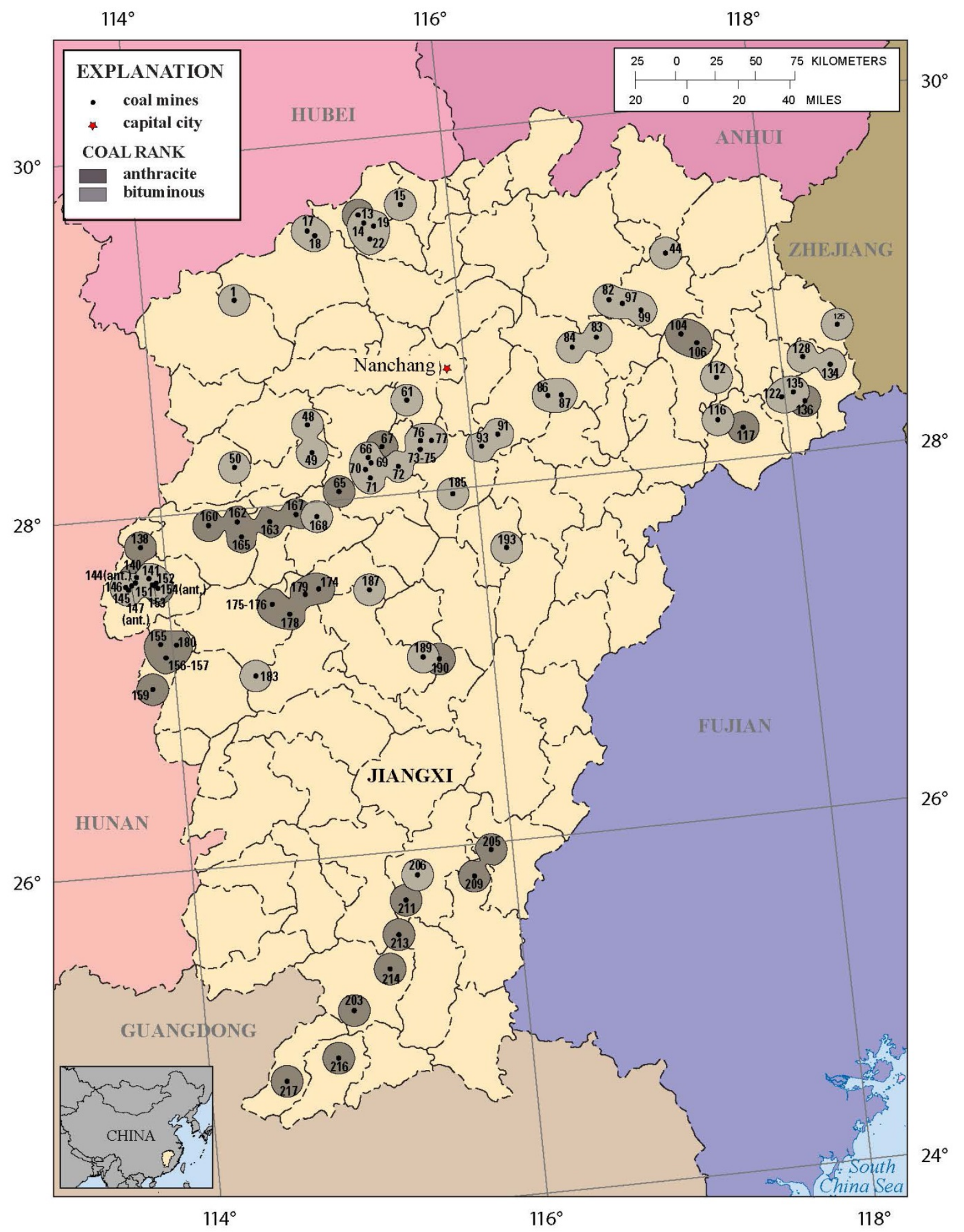

Figure 17. Coal mines and coal-mining areas of Jiangxi Province. Numbers are ID numbers in table 1. Abbreviation used: ant. - anthracite. Coal-mining areas are within 10-kilometer buffers of mine locations. 


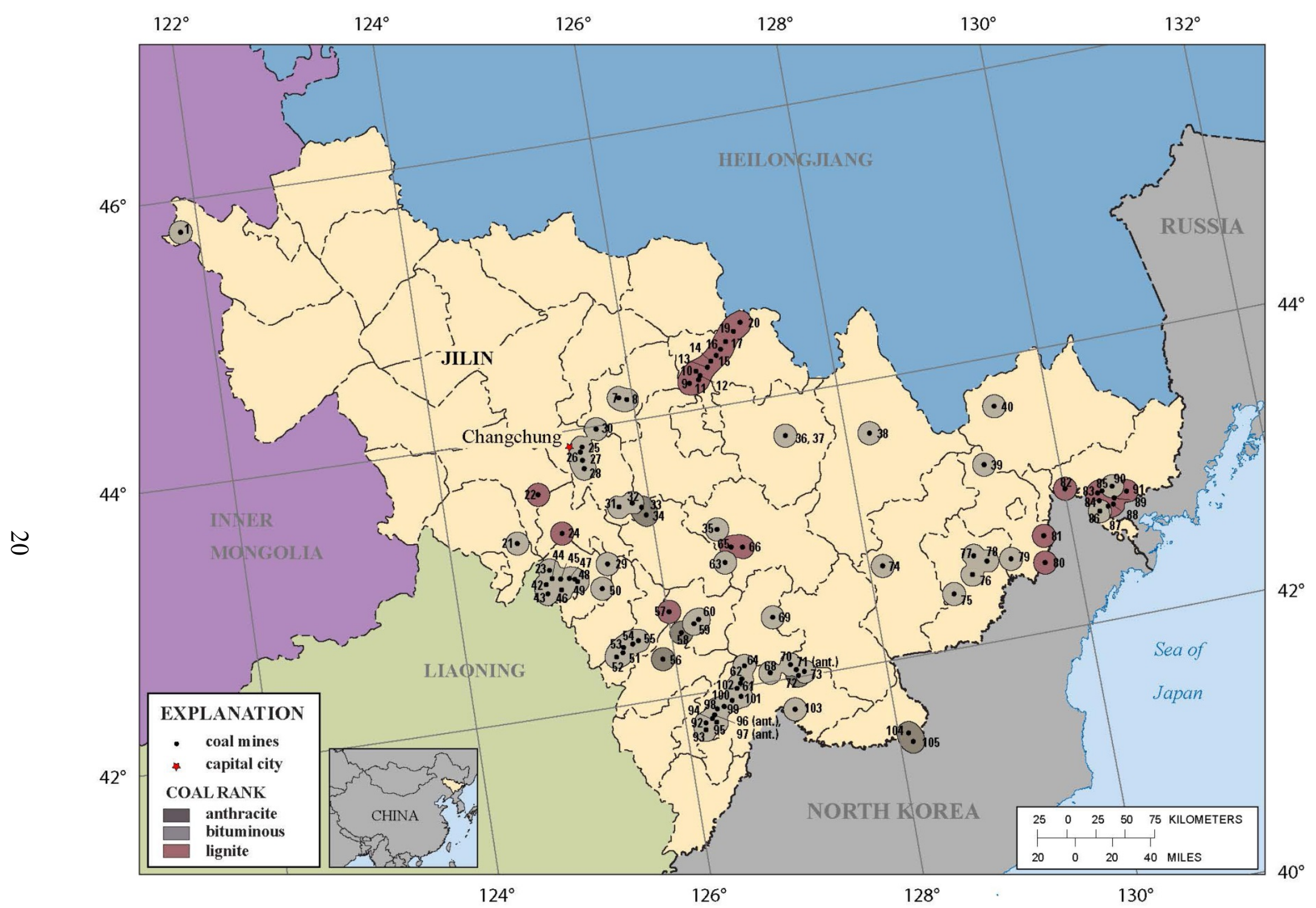

Figure 18. Coal mines and coal-mining areas of Jilin Province. Numbers are ID numbers in table 1. Abbreviation used: ant. - anthracite. Coalmining areas are within 10-kilometer buffers of mine locations. 


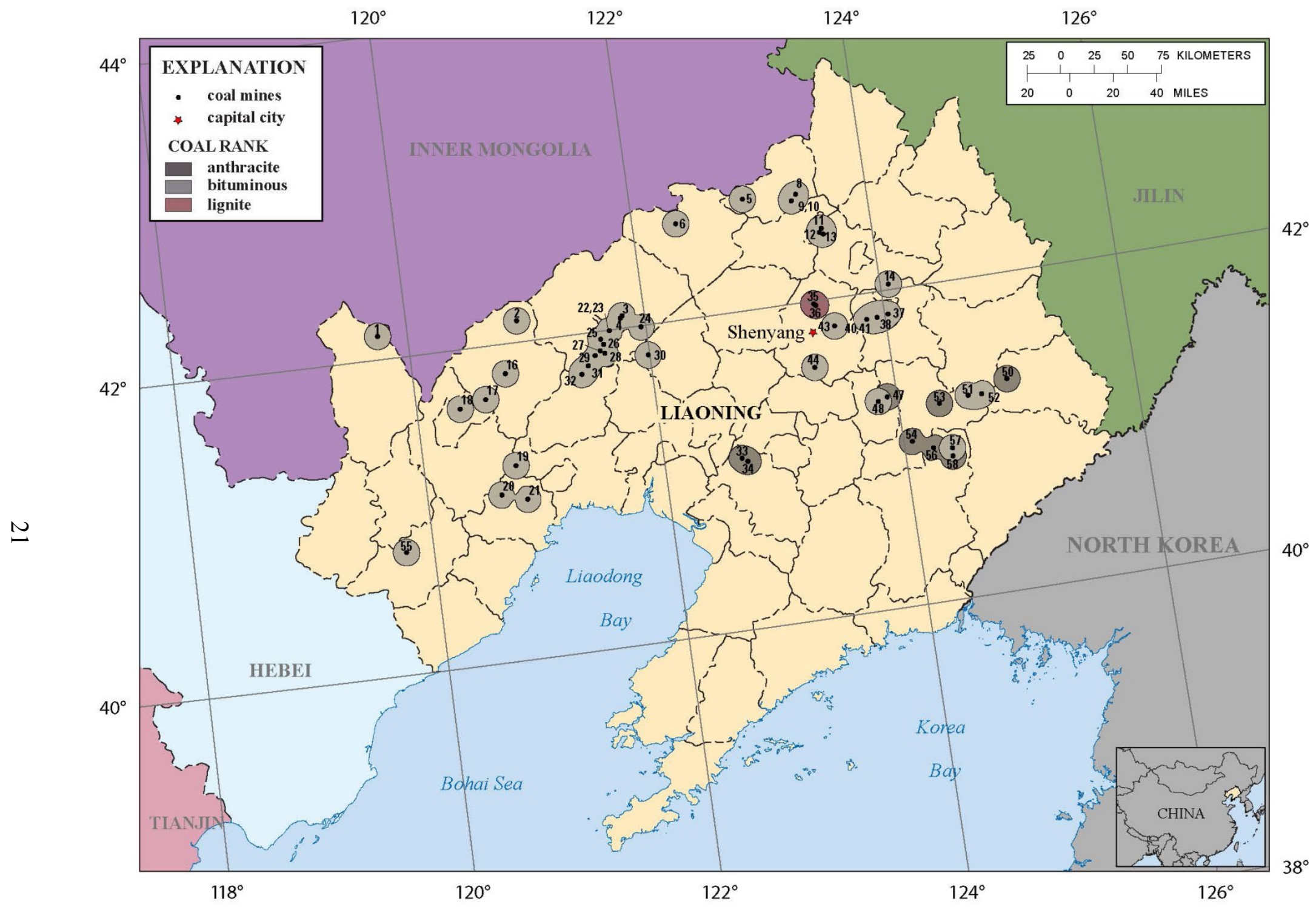

Figure 19. Coal mines and coal-mining areas of Liaoning Province. Numbers are ID numbers in table 1. Coal-mining areas are within 10-kilometer buffers of mine locations. 


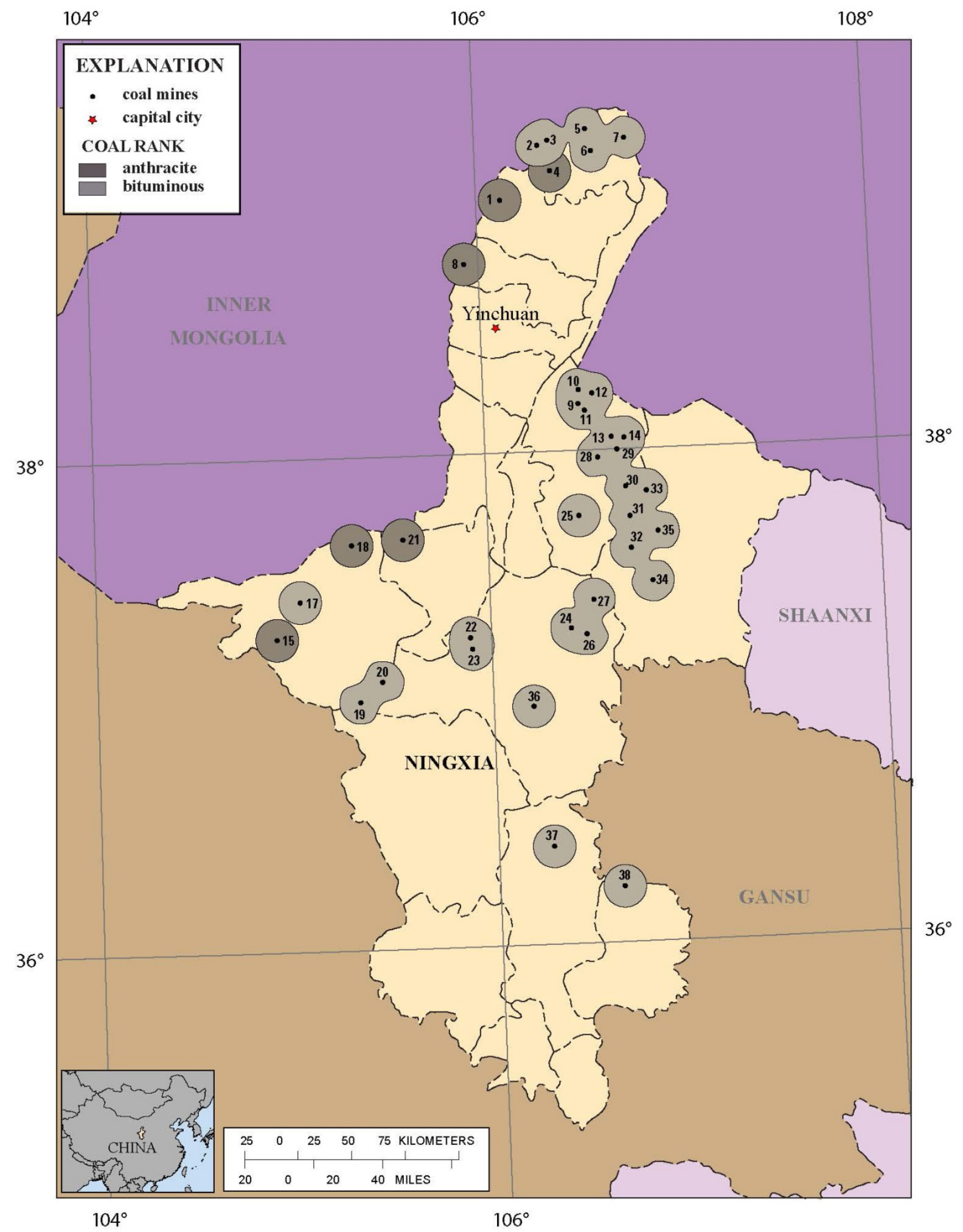

Figure 20. Coal mines and coal-mining areas of Ninxia Hui Autonomous Region. Numbers are ID numbers in table 1. Coal-mining areas are within 10-kilometer buffers of mine locations. 
$90^{\circ}$

$92^{\circ}$

$94^{\circ}$

$96^{\circ}$

$98^{\circ}$

$100^{\circ}$

$102^{\circ}$

EXPLANATION

- coal mines

* capital city

COAL RANK

anthracite
bituminous

lignite

$36^{\circ}$
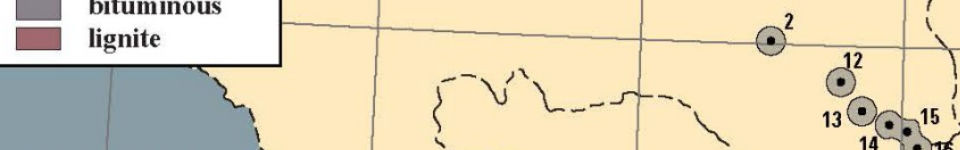

. $40-0.90$

${ }_{13} \cdot 015,15 \%$
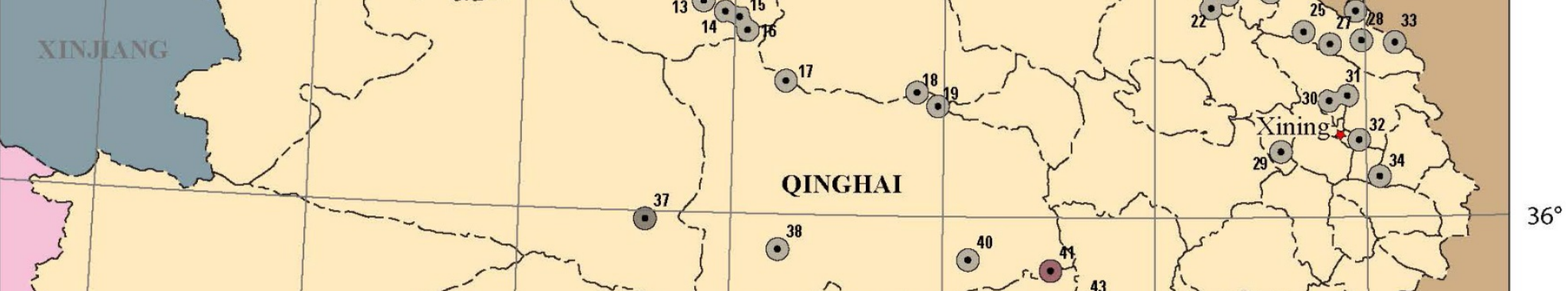

$34^{\circ}$
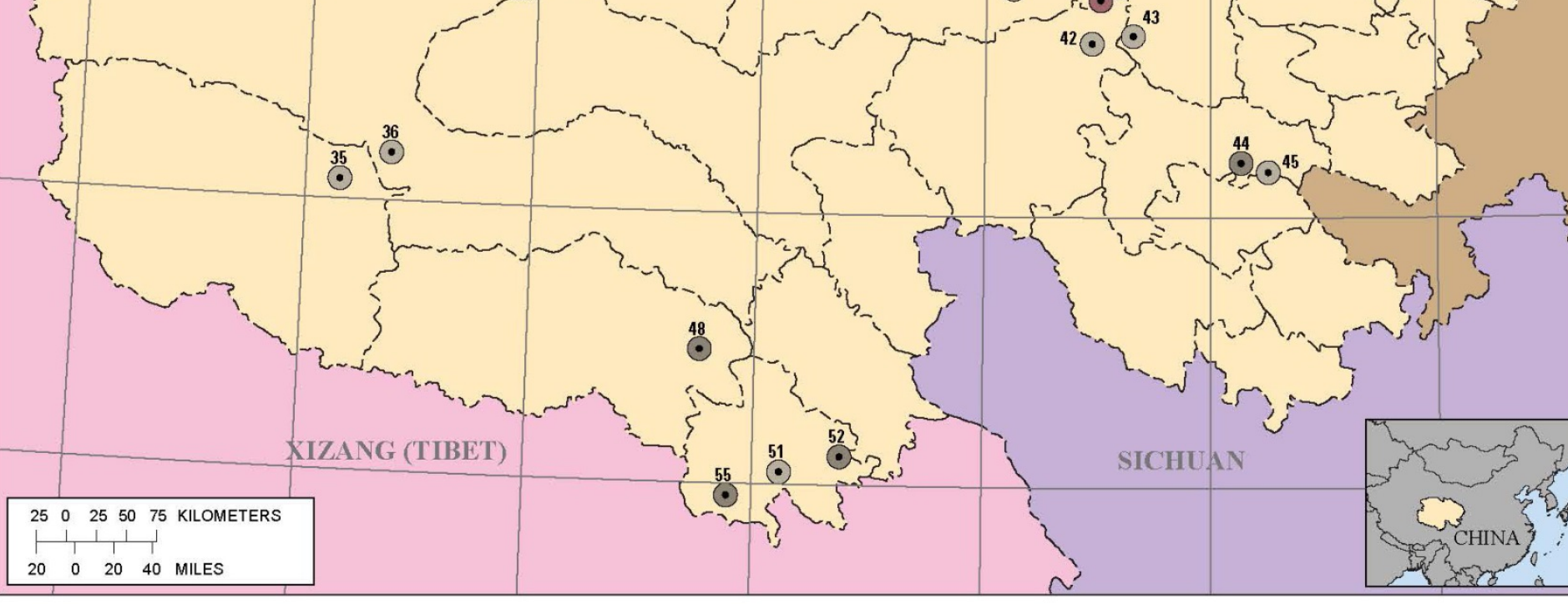

$90^{\circ}$

$92^{\circ}$

$94^{\circ}$

$96^{\circ}$

$102^{\circ}$

Figure 21. Coal mines and coal-mining areas of Qinghai Province. Numbers are ID numbers in table 1. Coal-mining areas are within 10-kilometer buffers of mine locations. 


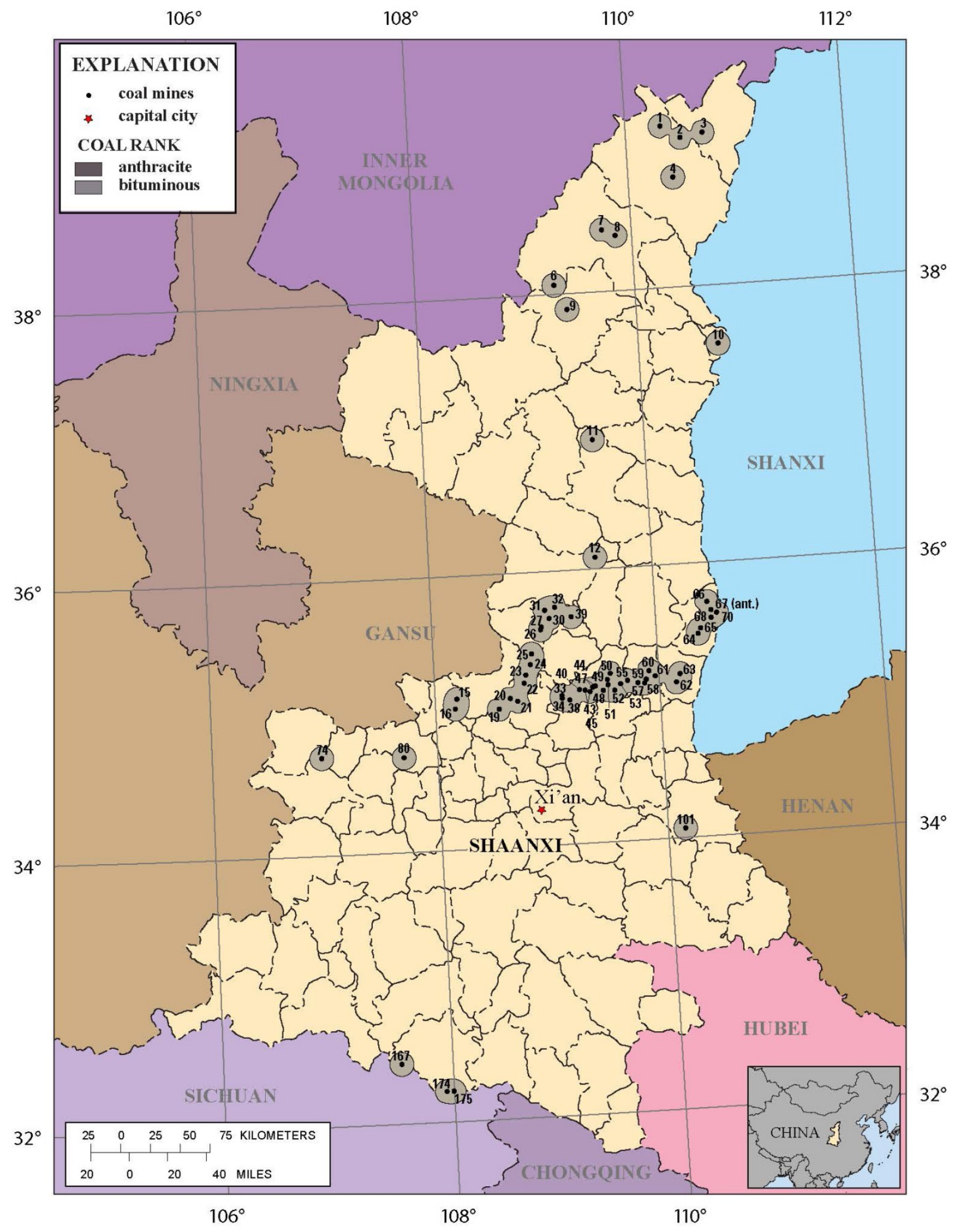

Figure 22. Coal mines and coal-mining areas of Shaanxi Province. Numbers are ID numbers in table 1. Abbreviation used: ant. - anthracite. Coal-mining areas are within 10-kilometer buffers of mine locations. 


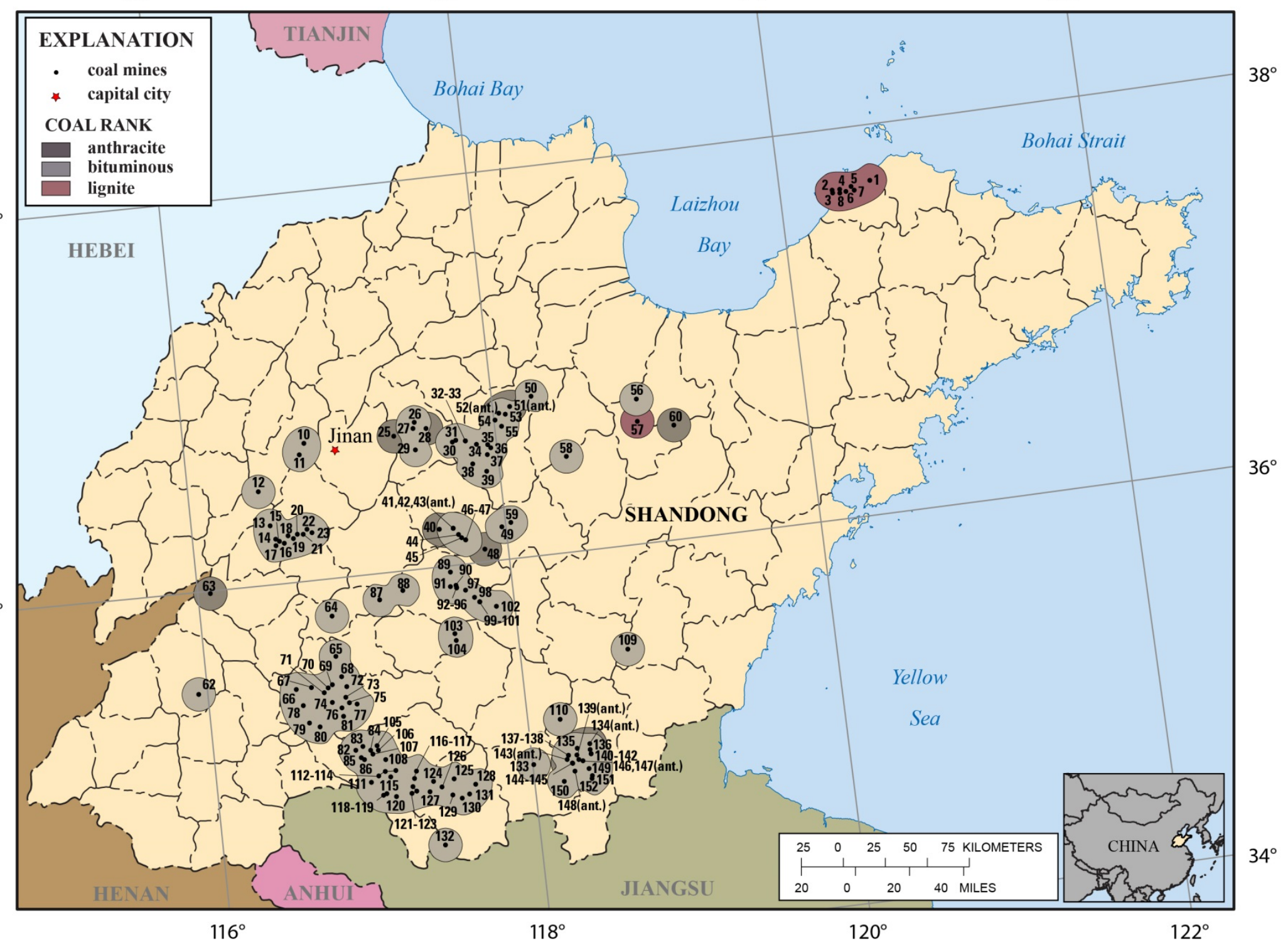

Figure 23. Coal mines and coal-mining areas of Shandong Province. Numbers are ID numbers in table 1. Abbreviation used: ant. - anthracite. Coal-mining areas are within 10-kilometer buffers of mine locations. 


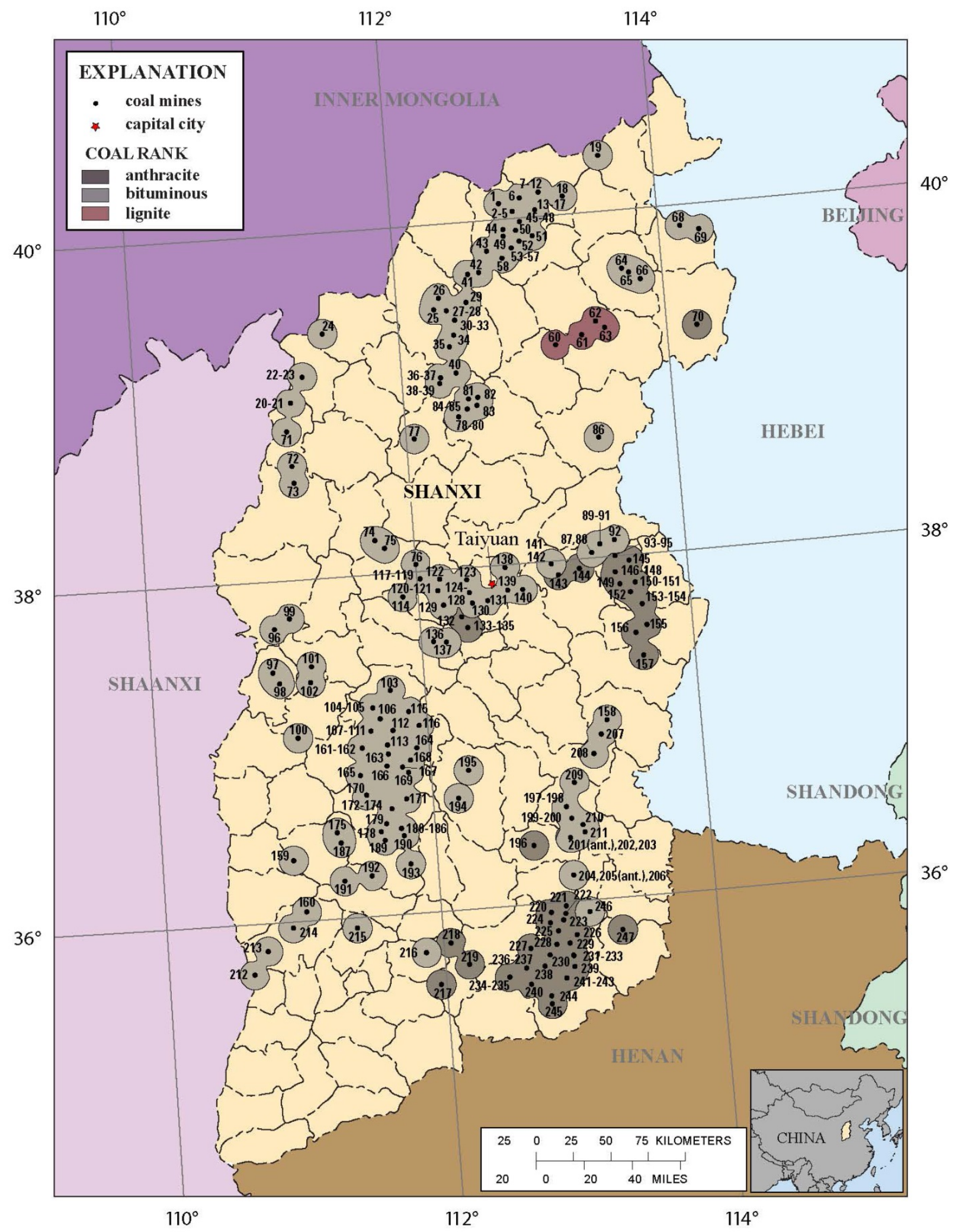

Figure 24. Coal mines and coal-mining areas of Shanxi Province. Numbers are ID numbers in table 1. Abbreviation used: ant. - anthracite. Coal-mining areas are within 10-kilometer buffers of mine locations. 


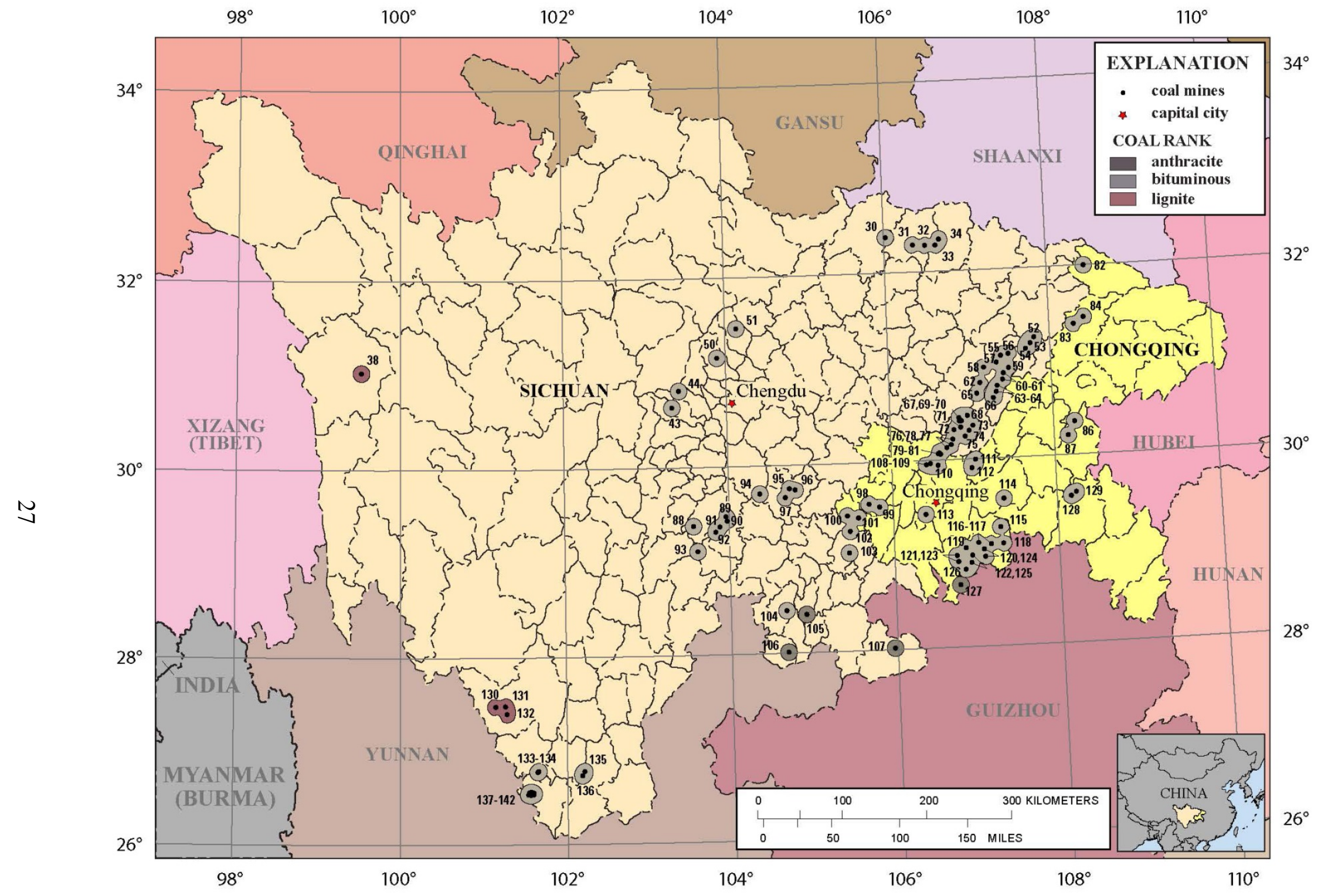

Figure 25. Coal mines and coal-mining areas of Sichuan Province and Chongqing Municipality. Numbers are ID numbers in table 1. Coal-mining areas are within 10-kilometer buffers of mine locations. 


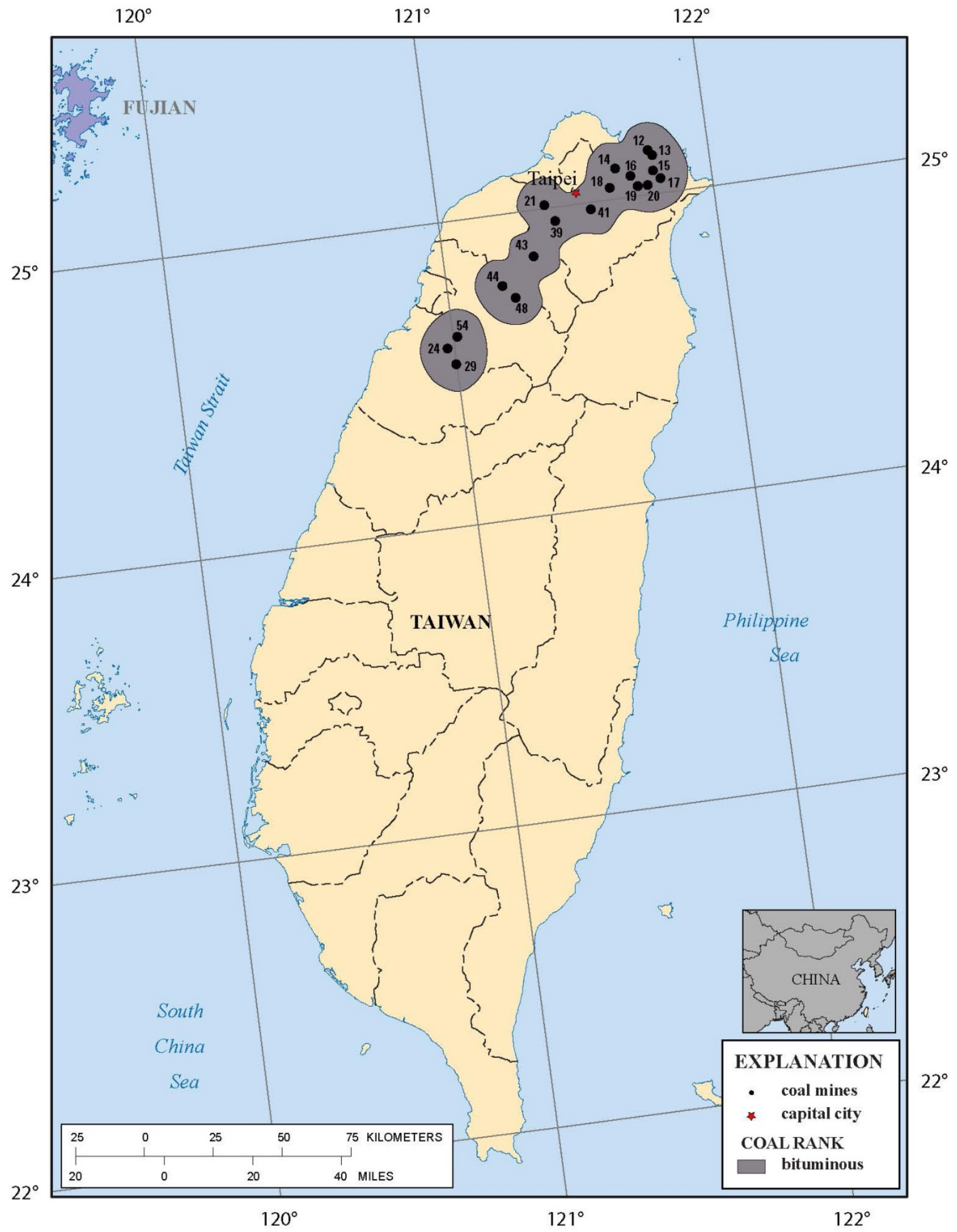

Figure 26. Coal mines and coal-mining areas of Taiwan. Numbers are ID numbers in table 1. Coal-mining areas are within 10-kilometer buffers of mine locations. 


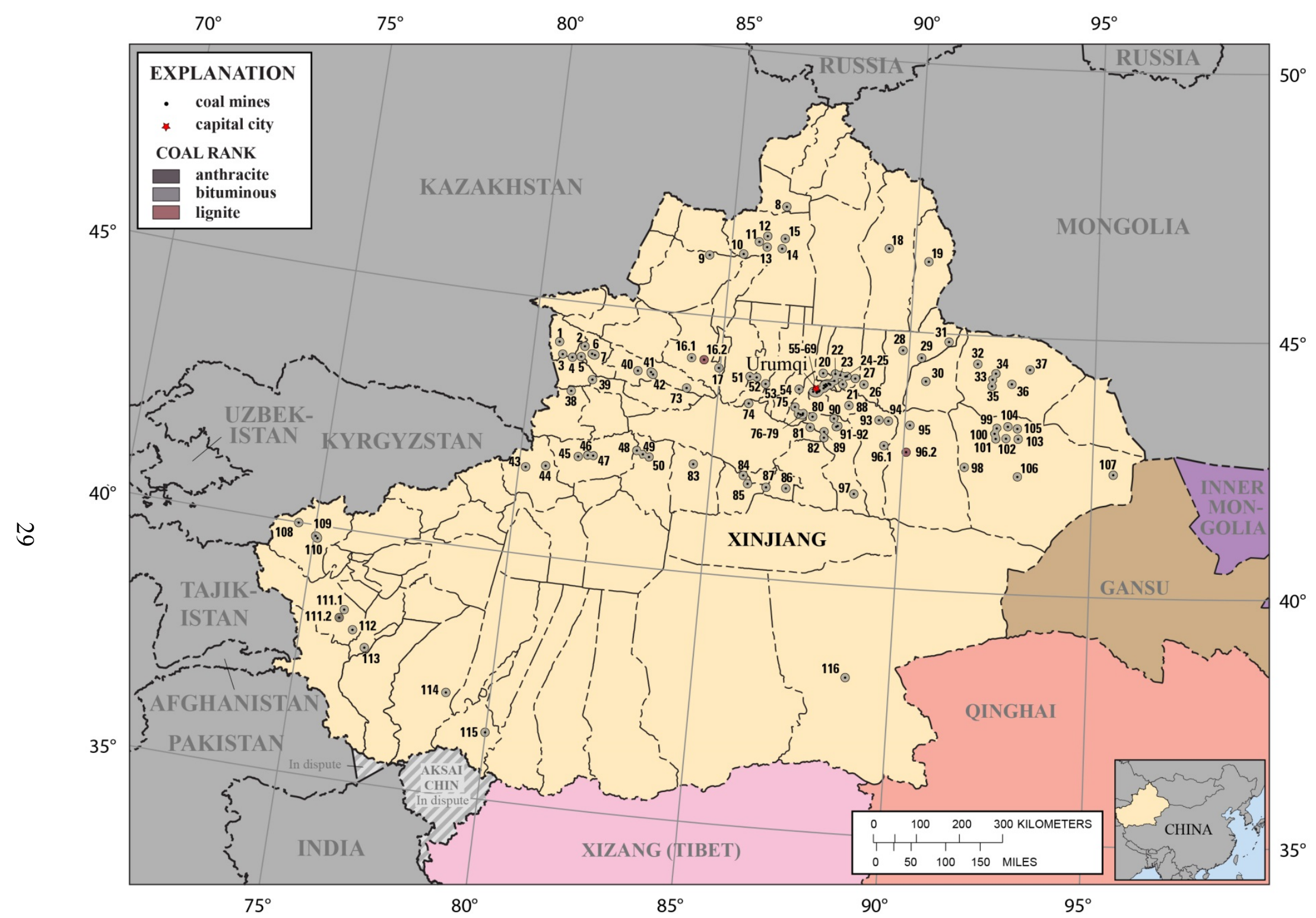

Figure 27. Coal mines and coal-mining areas of Xinjiang Uyghur Autonomous Region. Numbers are ID numbers in table 1. Coal-mining areas are within 10-kilometer buffers of mine locations. 


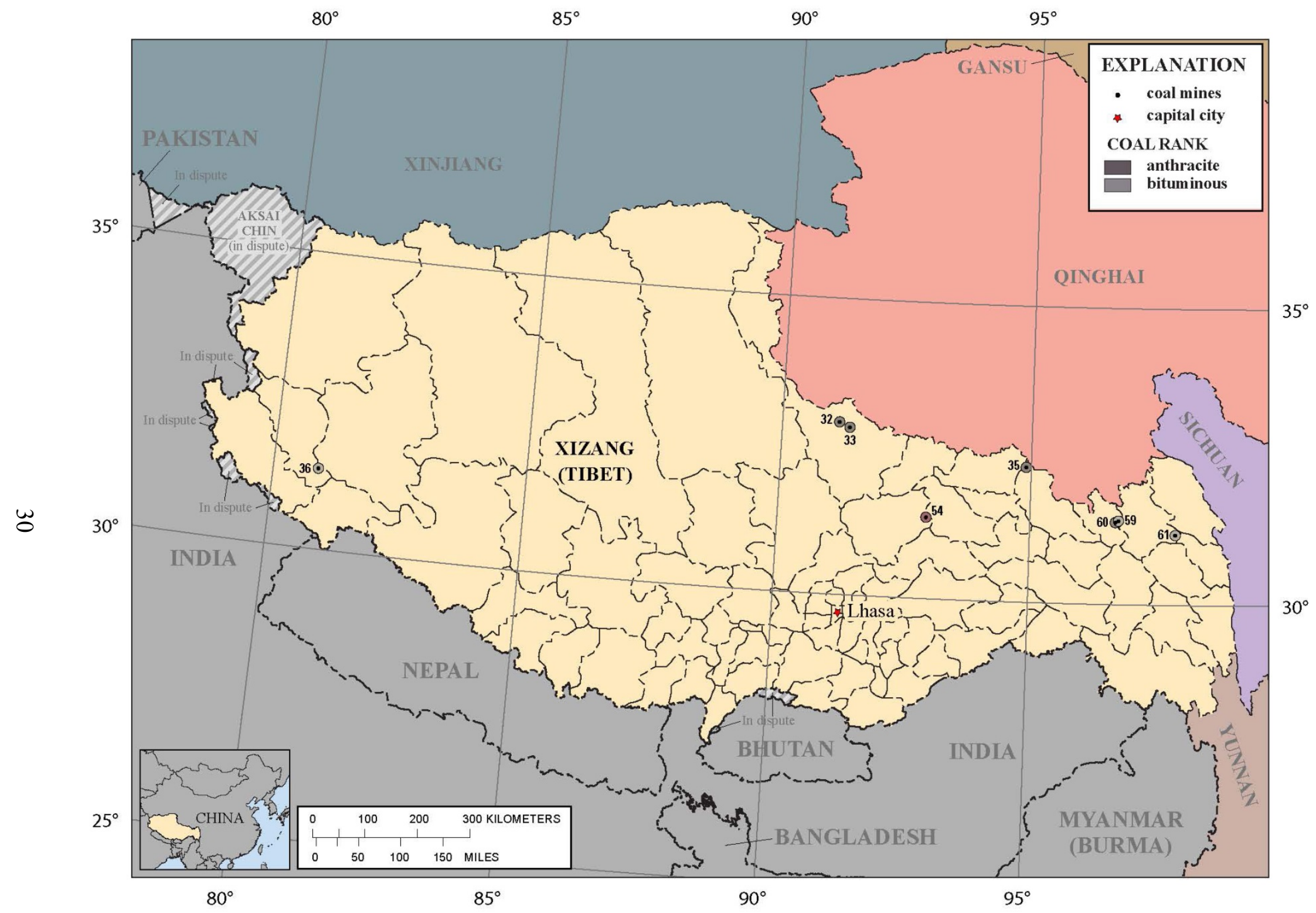

Figure 28. Coal mines and coal-mining areas of Xizang (Tibet) Autonomous Region. Numbers are ID numbers in table 1. Coal-mining areas are within 10-kilometer buffers of mine locations. 


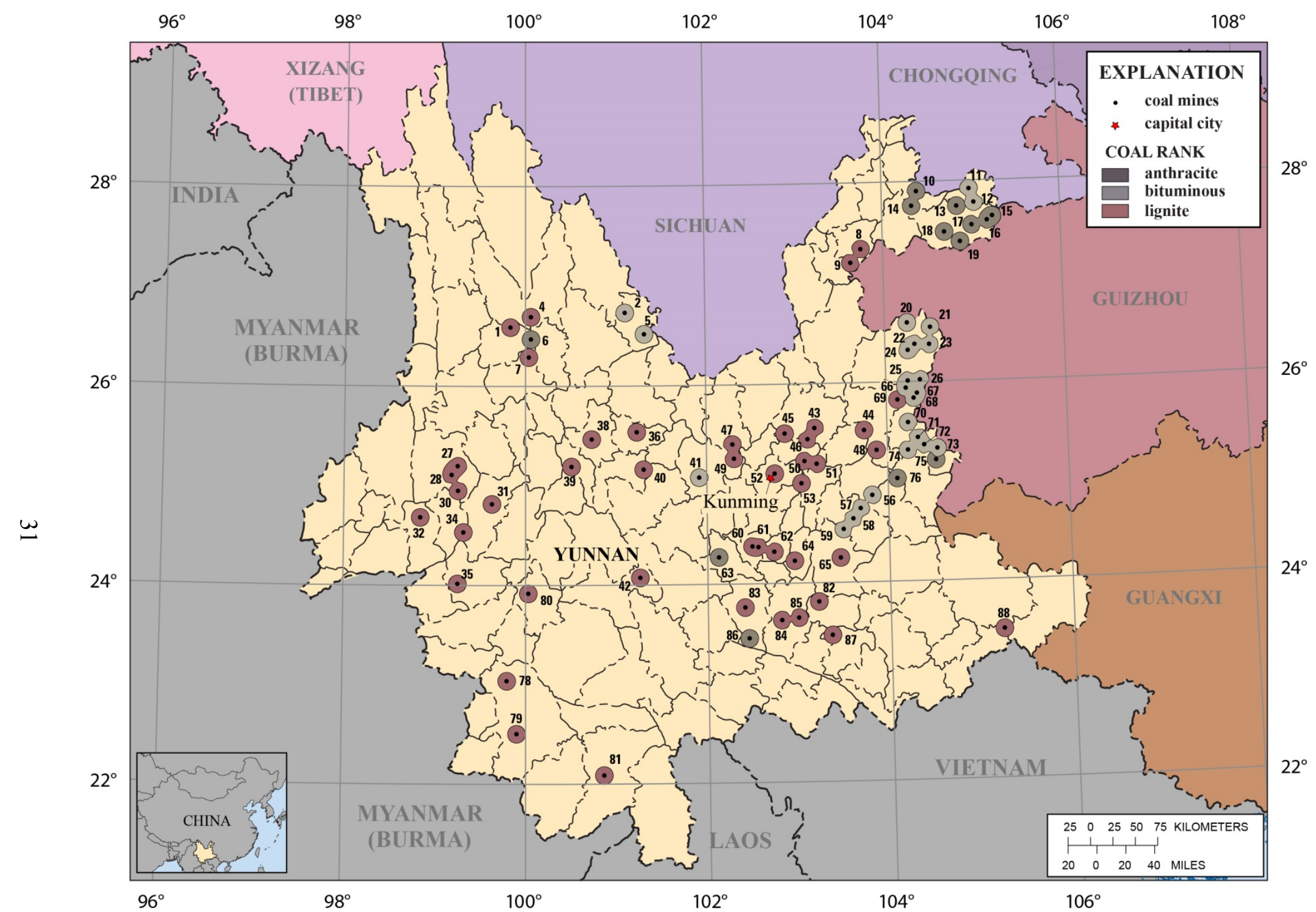

Figure 29. Coal mines and coal-mining areas of Yunnan Province. Numbers are ID numbers in table 1. Coal-mining areas are within 10-kilometer buffers of mine locations. 


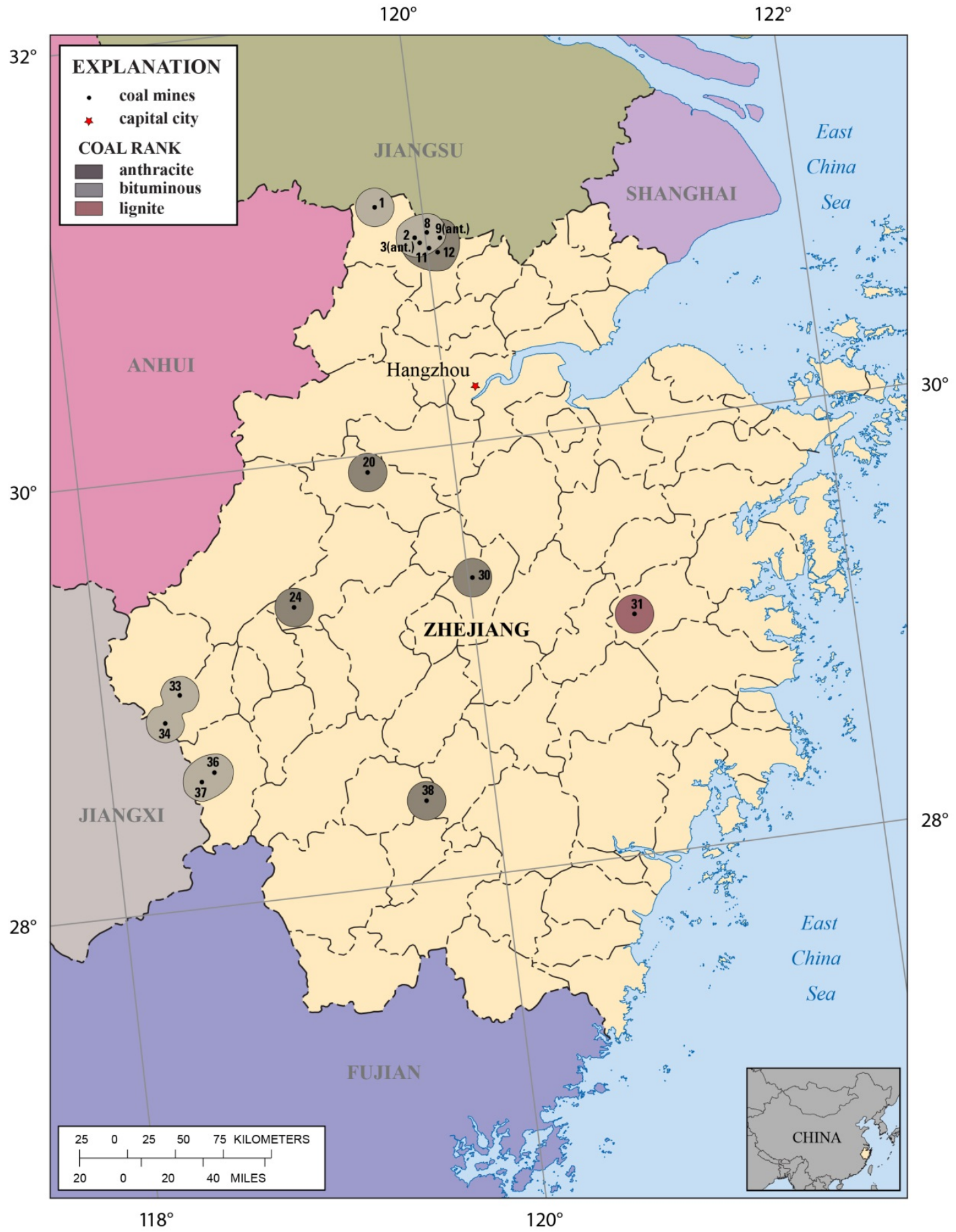

Figure 30. Coal mines and coal-mining areas of Zhejiang Province. Numbers are ID numbers in table 1. Abbreviation used: ant. - anthracite. Coal-mining areas are within 10-kilometer buffers of mine locations.

In addition, coal-field outlines from a previously published map by Dai and others (2012) were also digitized and are available for download as a separate GIS data file (fig. 31). The accompanying metadata file "AllChinaCoalMiningAreas Metadata.htm" describes GIS processing used to create this file. 


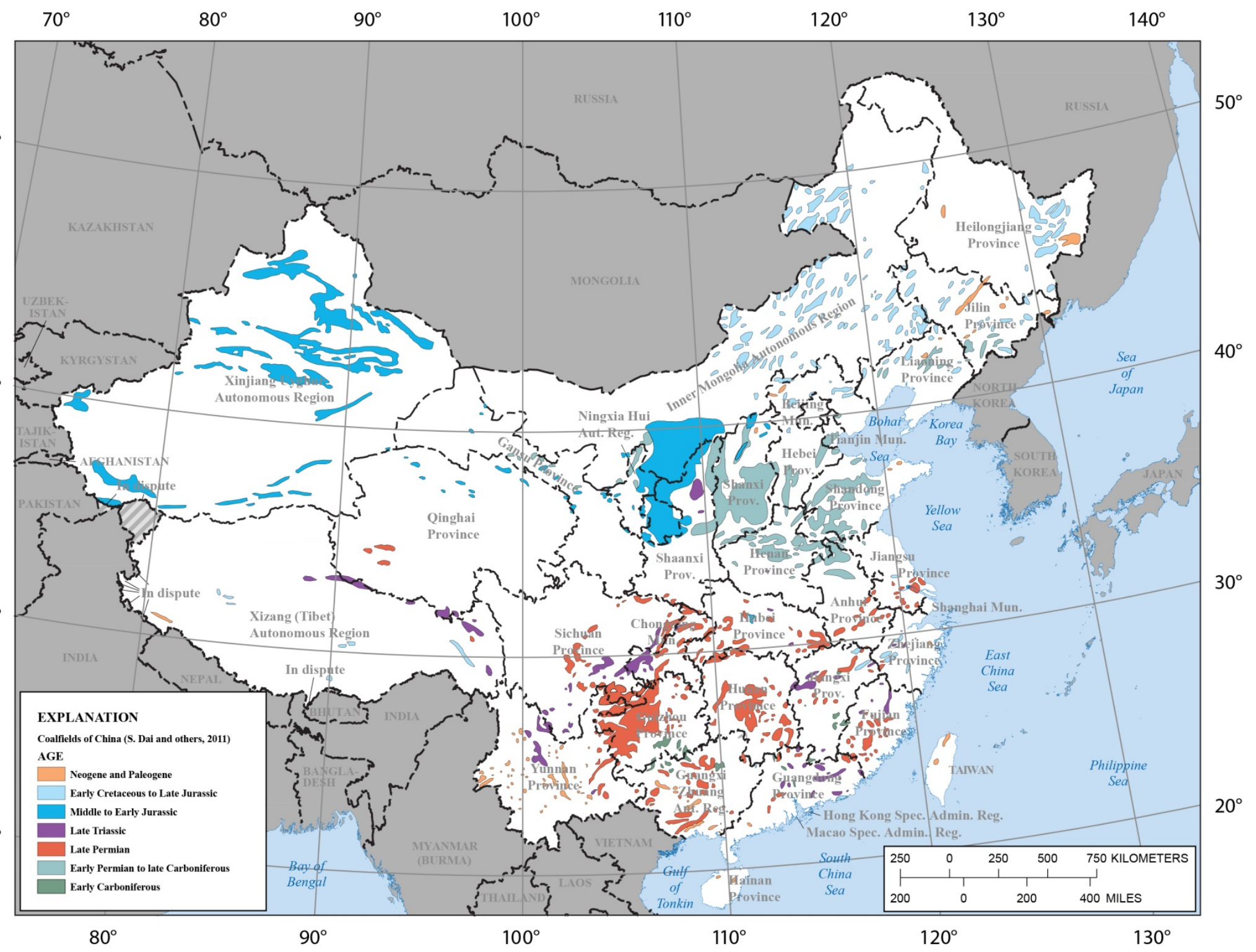

Figure 31. Coal fields of China classified by age (from Dai and others, 2012). Abbreviations used: Aut. Reg. - Autonomous Region; Mun. - Municipality; Prov. - Province; Spec. Admin. Reg. - Special Administrative Region. 
In general, the areas depicting regions of coal-mining and coal fields should not be used for the calculation of resources. International political boundaries and shorelines used in these figures come from shapefiles downloaded from the U.S. Department of State (2011). Province- and district-level boundaries were created by the USGS for an earlier unpublished project (H. Belkin, USGS, unpub. data, 2006) from the same source as the coal mine locations (Wang, 2001).

\section{Chemistry of Coal Samples}

Chemical data for 332 coal samples from China (328) and Taiwan (4) from a previous USGS study of world coal quality (Tewalt and others, 2010) are included in a GIS point shapefile with an accompanying metadata file called "ChinaAnalyticalData Metadata.htm."

The samples were collected from the highest-producing coal mines; 327 samples are run-of-mine or channel samples, of which 22 are washed and (or) cleaned samples. Locations of these points are displayed on figure 32. Chemical attributes include proximate and ultimate analyses, sulfur-form data, ash-fusion temperatures, and free-swelling indices from a commercial laboratory, and major-, minor-, and trace-element abundances from a USGS laboratory. All samples were analyzed between 2002 and 2005. All chemical analyses and physical property determinations were done using routine American Society for Testing and Materials (ASTM) methods. Descriptive data for the samples include the mine name, operator, stratigraphic unit and location. About 95 percent of coal production in China is from underground mining (Tien, 2006) and about 25 percent of all samples were collected from the Shanxi province, one of the largest provincial producers of coal. Only six samples were collected from Xinjiang Uygur Autonomous Region, which, although it accounts for 55 percent of total China coal reserves, is remote and largely undeveloped (Collins and Erickson, 2012). Predominantly Paleozoic and Mesozoic coals are mined, especially in eastern China, and the distribution of rank between collected samples is approximately 80 percent bituminous or subbituminous, 15 percent anthracite, and 5 percent lignite. 


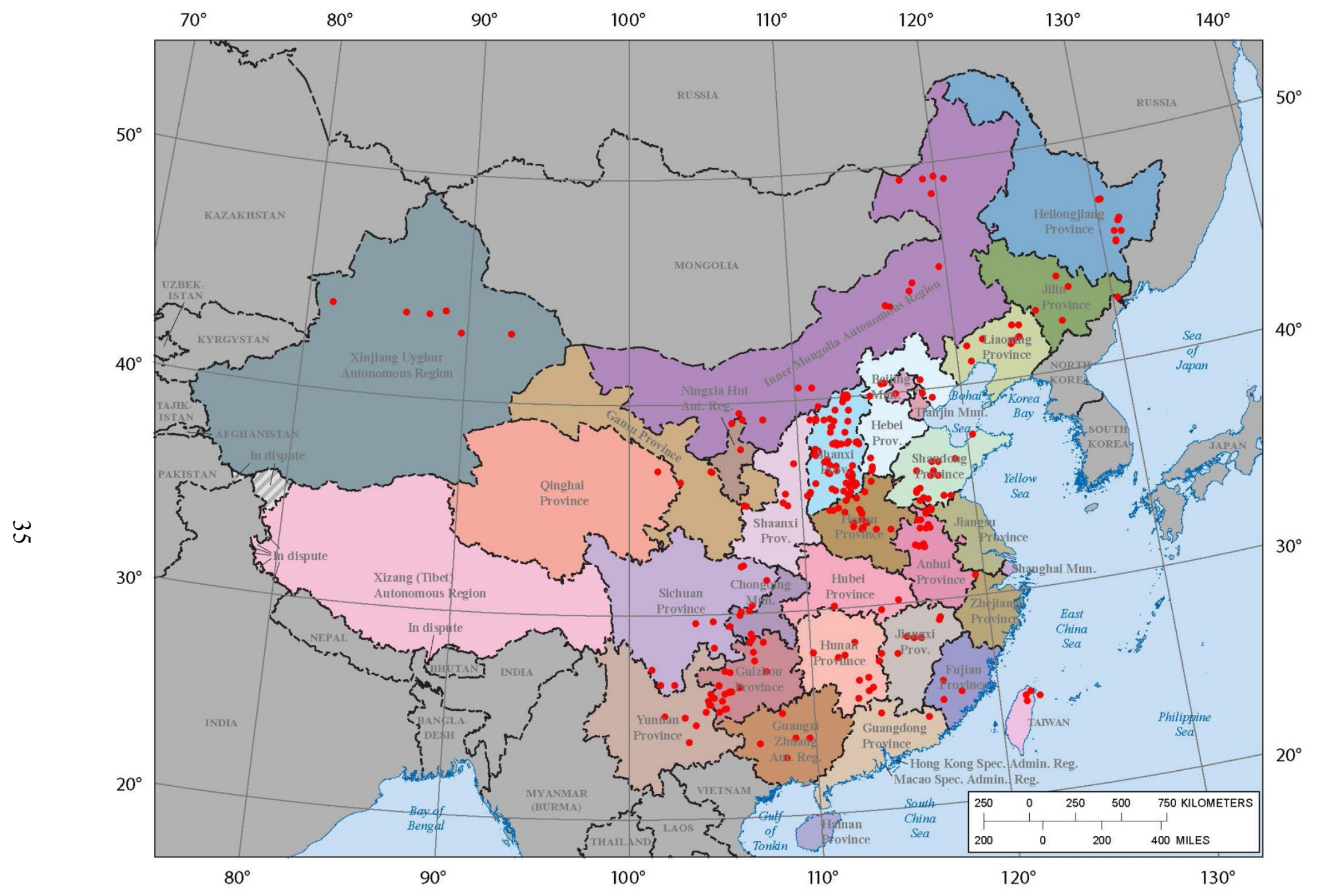

Figure 32. Locations of coal chemistry samples (red closed circles). Abbreviations used: Aut. Reg. - Autonomous Region; Mun. - Municipality; Prov. - Province; Spec. Admin. Reg. - Special Administrative Region. 
Previous publications (Belkin and others, 2000; Belkin and Hower, 2012; Ding and others, 2003 ) indicated links between coal chemical composition, usage, and human health impacts within China. Furthermore, a nationwide coal chemical database generated by ASTM (or similar) analytical standards was not available. Such a database could be useful for assessing coal-quality parameters of coal mined in China. To this end, Robert B. Finkelman (USGS, retired) contracted with Professor Baoshan Zheng (Institute of Geochemistry, Chinese Academy of Sciences, Guiyang City, Guizhou Province) to collect coal from the major mines. In 2001, Professor Zheng was assisted by Dr. Daishe $\mathrm{Wu}$, Dr. Shehong Li, Dr. Jianming Zhu, and Dr. Binbin Wang, all of the Institute of Geochemistry. The total collection of this coal was approximately 950 kilograms and is denoted by a sample number beginning with "CN." The locations of these samples were verified by Martina S. Hopkins (formerly with the USGS) in 2005.

\section{References Cited}

Belkin, H.E., and Hower, J.C., 2012, High-iron chamosite in bituminous coals in Xuanwei County, China; A possible contributing factor to a high lung cancer rate [abs.] in V.M. Goldschmidt Conference, 22d, Montreal, Canada, June 24-29, 2012: Mineralogical Magazine, v. 76, p. 1468, accessed August 7, 2014 at http://www.minersoc.org/goldschmidt2012/docs/e120624aabstract03092.doc.

Belkin, H.E., Zheng, Baoshan, and Finkelman, R.B., 2000, Human health effects of domestic combustion of coal in rural China-A causal factor for arsenic and fluorine poisoning in [Proceedings] The Second World Chinese Conference on Geologic Sciences: Palo Alto, Calif., Stanford University, p. A-522 to A-524.

British Petroleum, 2011, Statistical review of world energy, June 2011: British Petroleum Web page, accessed October 7, 2011, at http://bp.com/statisticalreview.

China Daily, 2011, Asia's largest coal reserve discovered in Xinjiang: China Daily Web page, accessed August 2, 2012, at http://www.chinadaily.com.cn/bizchina/2011-12/23/content_14316700.htm.

Collins, Gabe, and Erickson, Andrew, 2012, Xinjiang poised to become China's largest coal producer; Will move global coal, natural gas, and crude oil markets: China SignPost Web page, September 21, 2012, accessed August 7, 2014 at http://www.chinasignpost.com/2012/09/21/xinjiang-poised-tobecome-chinas-largest-coal-producer-will-move-global-coal-natural-gas-and-crude-oil-markets/.

Dai, S., Ren, D., Chou, C., Finkelman, R.B., Seredin, V.V., and Zhou, Y., 2012, Geochemistry of trace elements in Chinese coals; A review of abundances, genetic types, impacts on human health, and industrial utilization: International Journal of Coal Geology, v. 94, p. 3-21. [Also available at http://dx.doi.org/10.1016/j.coal.2011.02.003.]

Ding, Z., Zheng, B., Finkelman, R.B., Belkin, H.E., Hu, T., and Liu, T., 2003, Application of XAFS and mossbauer spectroscopy in studying the modes of occurrence of arsenic and iron in high-As coals from Southwest Guizhou Province: Geological Journal of China Universities, v. 9, no. 2, p. 273-278. [Also available at http://caod.oriprobe.com/articles/5804288/Application_of_XAFS_and_Mossbauer_Spectroscopy_in_ Studying_the_Mode_of.htm.]

Energy Information Administration, 2011, Country analysis briefs; China: Energy Information Administration Web page, accessed August 2, 2012, at http://www.eia.gov/cabs/china/Full.html.

Energy Information Administration, 2012, International Energy Statistics: Energy Information Administration Web page, accessed July 9, 2012, at http://www.eia.gov/cfapps/ipdbproject/iedindex3.cfm?tid=1\&pid=7\&aid=1\&cid=regions\&syid=198 3 \&eyid $=2011$ \&unite $=$ TST. 
Tewalt, S.J., Belkin, H.E., SanFilipo, J.R., Merrill, M.D., Palmer, C.A., Warwick, P.D., Karlsen, A.W., Finkelman, R.B., and Park, A.J., compilers, 2010, Chemical analyses in the World Coal Quality Inventory, version 1: U.S. Geological Survey Open-File Report 2010-1196, 4 p. [Also available at http://pubs.usgs.gov/of/2010/1196/.]

Tien, J.C., 2006, Coalbed methane production and utilization in China, in Proceedings of the 2006 joint meeting of the Society for Mining and Engineering \& International Conference on Acid Rock Drainage: Englewood, Colo., Society for Mining and Engineering, CD-ROM. [Also available at http://www.onemine.org/search/summary.cfm/Coalbed-Methane-Production-And-Utilization-InChina?d=123456789012345678901234567890123456789012345678901234567890123439688.]

U.S. Department of State, 2011, Intelink (restricted Web site), accessed April, 2010, at https://www.intelink.gov.

Wang, Yongqin, 2001, Atlas of solid fuel and nonmetal resources of China: Beijing, Geological Publishing House, $284 \mathrm{p}$.

World Energy Council, 2010, World Energy Insight 2010; 21st World Energy Congress report: World Energy Council Web page, accessed August 5, 2011, at http://www.worldenergy.org/documents/wec_combined.pdf.

World Coal Association, 2011, Xinjiang Province: World Coal Association Web site, accessed May 30, 2013, at http://www.worldcoal.org/resources/ecoal-archive/ecoal-current-issue/xinjiang-province/. 
Table 1. List of coal mines in China.

[Abbreviations used: dec. deg. - decimal degrees; NA - not available or not applicable]

\begin{tabular}{|c|c|c|c|c|c|c|c|c|}
\hline $\begin{array}{c}\text { ID } \\
\text { Number }\end{array}$ & $\begin{array}{c}\text { Province, National } \\
\text { Municipality, or } \\
\text { Autonomous Region }\end{array}$ & $\begin{array}{c}\text { Prefecture, Municipality, } \\
\text { Prefecture-Level City, or } \\
\text { League }\end{array}$ & $\begin{array}{l}\text { County, District or } \\
\text { County-Level City }\end{array}$ & $\begin{array}{l}\text { Latitude } \\
\text { (dec. deg.) }\end{array}$ & $\begin{array}{l}\text { Longitude } \\
\text { (dec. deg.) }\end{array}$ & Rank & $\begin{array}{l}\text { Relative } \\
\text { Mine Size }\end{array}$ & Mine Name \\
\hline 1 & Anhui Province & Suzhou City & Dangshan County & 34.332 & 116.483 & Bituminous & Large & Dang shan \\
\hline 2 & Anhui Province & Suzhou City & Xiao County & 34.250 & 116.597 & Bituminous & Small & Zhang datun \\
\hline 3 & Anhui Province & Suzhou City & Xiao County & 34.130 & 116.788 & Bituminous & Medium & Tang miao \\
\hline 4 & Anhui Province & Huaibei City & NA & 34.024 & 116.831 & Bituminous & Medium & Dai he \\
\hline 5 & Anhui Province & Huaibei City & NA & 34.062 & 116.888 & Bituminous & Medium & Shuo li \\
\hline 6 & Anhui Province & Huaibei City & NA & 34.039 & 116.920 & Bituminous & Medium & Shi tai tzu \\
\hline 7 & Anhui Province & Suzhou City & Xiao County & 34.051 & 116.943 & Bituminous & Small & Yong gu \\
\hline 8 & Anhui Province & Suzhou City & Xiao County & 34.249 & 116.949 & Bituminous & Small & Yian shan \\
\hline 9 & Anhui Province & Huaibei City & NA & 34.187 & 116.982 & Bituminous & Small & Meng zhuang \\
\hline 10 & Anhui Province & Huaibei City & NA & 34.125 & 116.973 & Bituminous & Small & Mao ying zi \\
\hline 11 & Anhui Province & Huaibei City & NA & 34.155 & 116.962 & Bituminous & Small & Yuan zhuang \\
\hline 12 & Anhui Province & Huaibei City & NA & 34.200 & 116.990 & Bituminous & Small & Shen zhuang \\
\hline 13 & Anhui Province & Huaibei City & NA & 33.514 & 116.029 & Bituminous & Large & Hua gou \\
\hline 14 & Anhui Province & Bozhuou City & Woyang County & 33.572 & 116.282 & Bituminous & Medium & Long shan \\
\hline 15 & Anhui Province & Huaibei City & Suixi County & 33.818 & 116.487 & Anthracite & Large & Wo long hu \\
\hline 16 & Anhui Province & Huaibei City & Suixi County & 33.551 & 116.508 & Bituminous & Large & Yuan dian \\
\hline 17 & Anhui Province & Huaibei City & Suixi County & 33.650 & 116.581 & Bituminous & Large & Han cun \\
\hline 18 & Anhui Province & Huaibei City & Suixi County & 33.546 & 116.646 & Bituminous & Large & Tongting West \\
\hline 19 & Anhui Province & Huaibei City & Suixi County & 33.801 & 116.660 & Anthracite & Medium & Bai shan \\
\hline 20 & Anhui Province & Bozhuou City & Mengcheng County & 33.397 & 116.683 & Bituminous & Large & Xu tuan \\
\hline 21 & Anhui Province & Bozhuou City & Suixi County & 33.915 & 116.689 & Bituminous & Large & Liu qiao \\
\hline 22 & Anhui Province & Huaibei City & Suixi County & 33.900 & 116.720 & Bituminous & Medium & Liang hua yuan \\
\hline 23 & Anhui Province & Huaibei City & Suixi County & 33.553 & 116.743 & Bituminous & Large & Tong ting East \\
\hline 24 & Anhui Province & Huaibei City & Suixi County & 33.786 & 116.777 & Anthracite & Medium & Cai shan \\
\hline 25 & Anhui Province & Huaibei City & NA & 33.939 & 116.781 & Bituminous & Small & Li lou \\
\hline 26 & Anhui Province & Huaibei City & NA & 33.895 & 116.793 & Bituminous & Medium & Yang zhuang \\
\hline 27 & Anhui Province & Huaibei City & NA & 33.879 & 116.813 & Anthracite & Small & Lie shan \\
\hline 28 & Anhui Province & Huaibei City & NA & 33.954 & 116.859 & Bituminous & Medium & Zhu zhuang \\
\hline 29 & Anhui Province & Huaibei City & NA & 33.981 & 116.873 & Bituminous & Medium & Zhang zhuang \\
\hline 30 & Anhui Province & Huaibei City & Suixi County & 33.442 & 116.885 & Bituminous & Large & Shuang dui \\
\hline 31 & Anhui Province & Huaibei City & Suixi County & 33.775 & 116.884 & Anthracite & Small & Qian ling \\
\hline 32 & Anhui Province & Suzhou City & NA & 33.417 & 117.019 & Bituminous & Large & Su South \\
\hline 33 & Anhui Province & Suzhou City & NA & 33.621 & 117.139 & Bituminous & Large & Su East \\
\hline 35 & Anhui Province & Fuyang City & NA & 32.854 & 116.091 & Bituminous & Large & Kou zi ji \\
\hline
\end{tabular}


Table 1. List of coal mines in China.-Continued

[Abbreviations used: dec. deg. - decimal degrees; NA - not available or not applicable]

\begin{tabular}{|c|c|c|c|c|c|c|c|c|}
\hline $\begin{array}{c}\text { ID } \\
\text { Number }\end{array}$ & $\begin{array}{l}\text { Province, National } \\
\text { Municipality, or } \\
\text { Autonomous Region }\end{array}$ & $\begin{array}{l}\text { Prefecture, Municipality, } \\
\text { Prefecture-Level City, or } \\
\text { League }\end{array}$ & $\begin{array}{l}\text { County, District or } \\
\text { County-Level City }\end{array}$ & $\begin{array}{l}\text { Latitude } \\
\text { (dec. deg.) }\end{array}$ & $\begin{array}{l}\text { Longitude } \\
\text { (dec. deg.) }\end{array}$ & Rank & $\begin{array}{l}\text { Relative } \\
\text { Mine Size }\end{array}$ & Mine Name \\
\hline 36 & Anhui Province & Fuyang City & Yingshang County & 32.800 & 116.223 & Bituminous & Large & Liu zhuang \\
\hline 37 & Anhui Province & Bozhuou City & Lixin County & 32.915 & 116.343 & Bituminous & Large & Zhan gou \\
\hline 38 & Anhui Province & Huainan City & Fengtai County & 32.773 & 116.511 & Bituminous & Large & Zhang ji \\
\hline 39 & Anhui Province & Huainan City & Fengtai County & 32.839 & 116.540 & Bituminous & Large & Gu qiao \\
\hline 40 & Anhui Province & Huainan City & Fengtai County & 32.703 & 116.540 & Bituminous & Medium & Xin ji \\
\hline 41 & Anhui Province & Huainan City & NA & 32.811 & 116.749 & Bituminous & Large & Pan ji shang \\
\hline 42 & Anhui Province & Huainan City & NA & 32.692 & 116.797 & Bituminous & Large & Kong ji \\
\hline 43 & Anhui Province & Huainan City & NA & 32.868 & 116.805 & Bituminous & Large & Pan ji North \\
\hline 44 & Anhui Province & Huainan City & NA & 32.603 & 116.752 & Bituminous & Large & Xie jia ji \\
\hline 45 & Anhui Province & Huainan City & NA & 32.598 & 116.887 & Bituminous & Small & Da tong \\
\hline 46 & Anhui Province & Chaohu City & Wuwei County & 31.042 & 117.603 & Anthracite & Small & Kun shan \\
\hline 48 & Anhui Province & Xuancheng City & Guangde County & 31.082 & 119.568 & Bituminous & Medium & Xin hang \\
\hline 49 & Anhui Province & Xuancheng City & Guangde County & 31.020 & 119.573 & Bituminous & Small & Du shan \\
\hline 50 & Anhui Province & Chizhou City & Guichi District & 30.412 & 117.257 & Bituminous & Small & Xin xin \\
\hline 51 & Anhui Province & Chizhou City & Guichi District & 30.451 & 117.397 & Anthracite & Small & Yin Pit \\
\hline 52 & Anhui Province & Tongling City & NA & 30.861 & 117.786 & Anthracite & Small & Sui shi ling \\
\hline 53 & Anhui Province & Tongling City & NA & 30.943 & 117.846 & Anthracite & Small & Xin miao \\
\hline 54 & Anhui Province & Tongling City & NA & 30.819 & 117.873 & Anthracite & Small & Da tong \\
\hline 55 & Anhui Province & Xuancheng City & Xuanzhou District & 30.743 & 118.721 & Bituminous & Small & Zhou wang \\
\hline 56 & Anhui Province & Xuancheng City & Xuanzhou District & 30.774 & 118.750 & Bituminous & Small & Xin tian \\
\hline 57 & Anhui Province & Xuancheng City & Ningguo City & 30.688 & 118.929 & Bituminous & Small & Gang kou \\
\hline 58 & Anhui Province & Xuancheng City & Ningguo City & 30.753 & 118.971 & Bituminous & Small & Hu lu shan \\
\hline 57 & Beijing Municipality & NA & Mentougou District & 39.993 & 116.131 & Coal & Medium & Yang wa \\
\hline 63 & Beijing Municipality & NA & Shunyi District & 40.078 & 116.943 & Bituminous & Small & Èr shi li chang shan \\
\hline 65 & Beijing Municipality & NA & Shunyi District & 40.073 & 116.906 & Coal & Small & San he \\
\hline 66 & Beijing Municipality & NA & Shunyi District & 40.065 & 116.945 & Coal & Small & Long ting hou \\
\hline 73 & Beijing Municipality & NA & Mentougou District & 39.978 & 115.682 & Coal & Small & Huang cheng yu \\
\hline 74 & Beijing Municipality & NA & Mentougou District & 39.973 & 115.734 & Coal & Small & Zhai tang hú lin \\
\hline 75 & Beijing Municipality & NA & Mentougou District & 39.970 & 115.998 & Coal & Small & Se shu fen \\
\hline 76 & Beijing Municipality & NA & Mentougou District & 39.967 & 115.979 & Coal & Medium & Wang ping cūn \\
\hline 77 & Beijing Municipality & NA & Mentougou District & 39.964 & 115.637 & Coal & Small & $\begin{array}{l}\text { Qing Shui-Qinglong } \\
\text { jian }\end{array}$ \\
\hline 79 & Beijing Municipality & NA & Mentougou District & 39.961 & 115.940 & Coal & Medium & Da tai \\
\hline 80 & Beijing Municipality & NA & Mentougou District & 39.954 & 115.703 & Coal & Medium & Hēitu gang \\
\hline
\end{tabular}


Table 1. List of coal mines in China.-Continued

[Abbreviations used: dec. deg. - decimal degrees; NA - not available or not applicable]

\begin{tabular}{|c|c|c|c|c|c|c|c|c|}
\hline $\begin{array}{c}\text { ID } \\
\text { Number }\end{array}$ & $\begin{array}{l}\text { Province, National } \\
\text { Municipality, or } \\
\text { Autonomous Region }\end{array}$ & $\begin{array}{l}\text { Prefecture, Municipality, } \\
\text { Prefecture-Level City, or } \\
\text { League }\end{array}$ & $\begin{array}{l}\text { County, District or } \\
\text { County-Level City }\end{array}$ & $\begin{array}{l}\text { Latitude } \\
\text { (dec. deg.) }\end{array}$ & $\begin{array}{l}\text { Longitude } \\
\text { (dec. deg.) }\end{array}$ & Rank & $\begin{array}{l}\text { Relative } \\
\text { Mine Size }\end{array}$ & Mine Name \\
\hline 81 & Beijing Municipality & NA & Mentougou District & 39.954 & 115.959 & Coal & Small & Anjia tan \\
\hline 82 & Beijing Municipality & NA & Mentougou District & 39.950 & 115.829 & Coal & Small & Cao jia pu \\
\hline 84 & Beijing Municipality & NA & Mentougou District & 39.938 & 115.918 & Coal & Medium & Muchengjian \\
\hline 85 & Beijing Municipality & NA & Mentougou District & 39.934 & 115.797 & Coal & Medium & Coal Pit \\
\hline 86 & Beijing Municipality & NA & Mentougou District & 39.932 & 115.856 & Coal & Medium & Qian jūn tai \\
\hline 87 & Beijing Municipality & NA & Mentougou District & 39.922 & 115.821 & Coal & Small & Dahan ling \\
\hline 89 & Beijing Municipality & NA & Mentougou District & 39.916 & 115.666 & Coal & Small & $\begin{array}{l}\text { Zhai tang ma lan - } \\
\text { hongshui yu }\end{array}$ \\
\hline 90 & Beijing Municipality & NA & Mentougou District & 39.907 & 115.776 & Coal & Medium & Chapeng ling \\
\hline 91 & Beijing Municipality & NA & Fangshan District & 39.904 & 115.761 & Coal & Large & $\begin{array}{l}\text { Da'an Mountain Coal } \\
\text { Mine, Yuangang } \\
\text { Well Field }\end{array}$ \\
\hline 92 & Beijing Municipality & NA & Fangshan District & 39.876 & 115.759 & Coal & Medium & $\begin{array}{l}\text { Da'an Mountain } \\
\text { Exploration Area }\end{array}$ \\
\hline 94 & Beijing Municipality & NA & Fangshan District & 39.847 & 115.648 & Coal & Small & Shi jia ying \\
\hline 100 & Beijing Municipality & NA & Fangshan District & 39.789 & 115.969 & Coal & Small & Wan fo tang \\
\hline 101 & Beijing Municipality & NA & Fangshan District & 39.785 & 115.915 & Coal & Medium & Fang shan \\
\hline 102 & Beijing Municipality & NA & Fangshan District & 39.777 & 115.540 & Coal & Small & Bao er shui \\
\hline 105 & Beijing Municipality & NA & Fangshan District & 39.732 & 115.879 & Coal & Medium & Zhang gou yu \\
\hline 107 & Beijing Municipality & NA & Fangshan District & 39.716 & 115.865 & Coal & Small & Zhang gou yu \\
\hline 116 & Beijing Municipality & NA & Haidian District & 39.972 & 116.192 & Coal & Small & Ba da chu \\
\hline 118 & Beijing Municipality & NA & Mentougou District & 39.948 & 116.073 & Coal & Small & Chengzi \\
\hline 119 & Beijing Municipality & NA & Mentougou District & 39.933 & 116.062 & Coal & Medium & Mentougou \\
\hline 123 & Beijing Municipality & NA & Mentougou District & 39.907 & 116.099 & Coal & Small & Feng cun \\
\hline 124 & Beijing Municipality & NA & Mentougou District & 39.905 & 116.014 & Coal & Small & Tan zhe si \\
\hline 127 & Beijing Municipality & NA & Mentougou District & 39.877 & 116.076 & Coal & Small & Wang cun \\
\hline 79 & Chongqing Municipality & NA & Hechuan District & 30.076 & 106.587 & Bituminous & Small & Sanhuiba No. 1 Mine \\
\hline 80 & Chongqing Municipality & NA & Hechuan District & 30.067 & 106.574 & Bituminous & Small & Sanhuiba No. 3 Mine \\
\hline 81 & Chongqing Municipality & NA & Hechuan District & 30.062 & 106.602 & Bituminous & Medium & Sanhuiba No. 2 Mine \\
\hline 82 & Chongqing Municipality & NA & Chengkou County & 31.995 & 108.462 & Bituminous & Small & Ju ma ping \\
\hline 83 & Chongqing Municipality & NA & Kai County & 31.383 & 108.301 & Bituminous & Small & Zi shui \\
\hline 84 & Chongqing Municipality & NA & Kai County & 31.450 & 108.428 & Bituminous & Small & No. 1 Coal Mine \\
\hline 86 & Chongqing Municipality & NA & Shizhu County & 30.351 & 108.257 & Bituminous & Small & Gan jia qiao \\
\hline 87 & Chongqing Municipality & NA & Shizhu County & 30.207 & 108.172 & Bituminous & Small & Lian hua si \\
\hline
\end{tabular}


Table 1. List of coal mines in China. - Continued

[Abbreviations used: dec. deg. - decimal degrees; NA - not available or not applicable]

\begin{tabular}{|c|c|c|c|c|c|c|c|c|}
\hline $\begin{array}{c}\text { ID } \\
\text { Number }\end{array}$ & $\begin{array}{l}\text { Province, National } \\
\text { Municipality, or } \\
\text { Autonomous Region }\end{array}$ & $\begin{array}{l}\text { Prefecture, Municipality, } \\
\text { Prefecture-Level City, or } \\
\text { League }\end{array}$ & $\begin{array}{l}\text { County, District or } \\
\text { County-Level City }\end{array}$ & $\begin{array}{l}\text { Latitude } \\
\text { (dec. deg.) }\end{array}$ & $\begin{array}{l}\text { Longitude } \\
\text { (dec. deg.) }\end{array}$ & Rank & $\begin{array}{l}\text { Relative } \\
\text { Mine Size }\end{array}$ & Mine Name \\
\hline 98 & Chongqing Municipality & NA & Yongchuan District & 29.560 & 105.707 & Bituminous & Small & Chang he bian \\
\hline 99 & Chongqing Municipality & NA & Dazu County & 29.525 & 105.834 & Bituminous & Small & Tang jia ba \\
\hline 100 & Chongqing Municipality & NA & Rongchang County & 29.442 & 105.440 & Bituminous & Small & Zeng jia shan \\
\hline 101 & Chongqing Municipality & NA & Rongchang County & 29.417 & 105.572 & Bituminous & Small & Shuang he No. 8 Well \\
\hline 108 & Chongqing Municipality & NA & NA & 29.955 & 106.423 & Bituminous & Small & Mo xin po \\
\hline 109 & Chongqing Municipality & NA & NA & 29.967 & 106.473 & Bituminous & Small & Yang liu ba \\
\hline 110 & Chongqing Municipality & NA & NA & 29.946 & 106.566 & Bituminous & Small & Liu jia gou \\
\hline 111 & Chongqing Municipality & NA & Changshou District & 29.994 & 107.025 & Bituminous & Small & Yang jia wan \\
\hline 112 & Chongqing Municipality & NA & Changshou District & 29.905 & 106.976 & Bituminous & Small & Bai yun si \\
\hline 113 & Chongqing Municipality & NA & NA & 29.429 & 106.394 & Bituminous & Medium & Zhong liang shan \\
\hline 114 & Chongqing Municipality & NA & Fuling District & 29.566 & 107.350 & Bituminous & Small & Zi li \\
\hline 115 & Chongqing Municipality & NA & Nanchuan District & 29.265 & 107.295 & Bituminous & Small & Sui jiang \\
\hline 116 & Chongqing Municipality & NA & NA & 29.107 & 107.020 & Bituminous & Small & Hong yan \\
\hline 117 & Chongqing Municipality & NA & Nanchuan District & 29.088 & 107.172 & Bituminous & Small & Nan chuan \\
\hline 118 & Chongqing Municipality & NA & Nanchuan District & 29.091 & 107.320 & Bituminous & Small & Dong sheng \\
\hline 119 & Chongqing Municipality & NA & NA & 29.056 & 106.865 & Bituminous & Medium & Cong lin \\
\hline 120 & Chongqing Municipality & NA & Nanchuan District & 29.035 & 107.086 & Bituminous & Small & Xian feng \\
\hline 121 & Chongqing Municipality & NA & NA & 28.978 & 106.753 & Bituminous & Small & Dong lin \\
\hline 122 & Chongqing Municipality & NA & NA & 28.976 & 106.940 & Bituminous & Small & Guan shi tai \\
\hline 123 & Chongqing Municipality & NA & NA & 28.913 & 106.769 & Bituminous & Small & $\begin{array}{l}\text { Nan tong Coal Mine, } \\
\text { Nos. } 1,2 \& 3 \text { Wells }\end{array}$ \\
\hline 124 & Chongqing Municipality & NA & Nanchuan District & 28.956 & 107.097 & Bituminous & Small & Hong guang \\
\hline 125 & Chongqing Municipality & NA & NA & 28.898 & 106.926 & Bituminous & Small & Yu tian bao \\
\hline 126 & Chongqing Municipality & NA & NA & 28.827 & 106.854 & Bituminous & Small & Xing long \\
\hline 127 & Chongqing Municipality & NA & Qijiang District & 28.665 & 106.782 & Anthracite & Medium & Shi hao \\
\hline 128 & Chongqing Municipality & NA & $\begin{array}{l}\text { Penshui Autonomous } \\
\text { County }\end{array}$ & 29.561 & 108.172 & Bituminous & Small & Cai jia ba Yu jiang \\
\hline 129 & Chongqing Municipality & NA & $\begin{array}{l}\text { Penshui Autonomous } \\
\text { County }\end{array}$ & 29.602 & 108.234 & Bituminous & Small & Yu jiang \\
\hline 1 & Fujian Province & Nanping City & Pucheng County & 28.133 & 118.487 & Anthracite & Small & Shan fang \\
\hline 2 & Fujian Province & Nanping City & Shaowu City & 27.294 & 117.571 & Anthracite & Small & \\
\hline 3 & Fujian Province & Nanping City & Wuyishan City & 27.667 & 118.045 & Anthracite & Small & Xia mei \\
\hline 4 & Fujian Province & Nanping City & Jianou City & 27.040 & 118.364 & Anthracite & Small & \\
\hline 5 & Fujian Province & Sanming City & Ninghua County & 26.408 & 116.908 & Anthracite & Small & Gan mu tan \\
\hline
\end{tabular}


Table 1. List of coal mines in China. - Continued

[Abbreviations used: dec. deg. - decimal degrees; NA - not available or not applicable]

\begin{tabular}{|c|c|c|c|c|c|c|c|c|}
\hline $\begin{array}{c}\text { ID } \\
\text { Number }\end{array}$ & $\begin{array}{c}\text { Province, National } \\
\text { Municipality, or } \\
\text { Autonomous Region }\end{array}$ & $\begin{array}{c}\text { Prefecture, Municipality, } \\
\text { Prefecture-Level City, or } \\
\text { League }\end{array}$ & $\begin{array}{l}\text { County, District or } \\
\text { County-Level City }\end{array}$ & $\begin{array}{l}\text { Latitude } \\
\text { (dec. deg.) }\end{array}$ & $\begin{array}{l}\text { Longitude } \\
\text { (dec. deg.) }\end{array}$ & Rank & $\begin{array}{l}\text { Relative } \\
\text { Mine Size }\end{array}$ & Mine Name \\
\hline 6 & Fujian Province & Sanming City & Qingliu County & 26.033 & 116.912 & Anthracite & Small & $\begin{array}{l}\text { Shang pin qiao } \\
\text { (Luo kuo) }\end{array}$ \\
\hline 7 & Fujian Province & Sanming City & Taining County & 26.734 & 116.999 & Anthracite & Small & Da bu \\
\hline 8 & Fujian Province & Sanming City & Mingxi County & 26.369 & 117.201 & Lignite & Small & Cheng guan \\
\hline 9 & Fujian Province & Sanming City & Mingxi County & 26.324 & 117.433 & Anthracite & Small & Niu jiao Pit \\
\hline 10 & Fujian Province & Sanming City & Jiangle County & 26.740 & 117.502 & Anthracite & Small & Lao hu shan \\
\hline 11 & Fujian Province & Sanming City & NA & 26.320 & 117.666 & Anthracite & Small & Bi kou \\
\hline 12 & Fujian Province & Sanming City & Datian County & 26.074 & 117.695 & Anthracite & Small & Xiao ji \\
\hline 13 & Fujian Province & Sanming City & Datian County & 26.024 & 117.755 & Anthracite & Small & Da zhu lin \\
\hline 14 & Fujian Province & Sanming City & Datian County & 26.113 & 117.809 & Anthracite & Small & Guang ping Pit Mouth \\
\hline 15 & Fujian Province & Sanming City & Datian County & 26.071 & 117.844 & Anthracite & Small & Qi tao \\
\hline 16 & Fujian Province & Sanming City & Sha County & 26.429 & 117.943 & Anthracite & Small & Duan xi \\
\hline 17 & Fujian Province & Longyan City & Liancheng County & 25.341 & 116.661 & Anthracite & Small & Miao qian \\
\hline 18 & Fujian Province & Longyan City & Liancheng County & 25.744 & 116.694 & Anthracite & Small & Xi shan \\
\hline 19 & Fujian Province & Longyan City & Liancheng County & 25.391 & 116.730 & Anthracite & Small & Jiang she \\
\hline 20 & Fujian Province & Sanming City & Qingliu County & 25.808 & 116.819 & Anthracite & Small & Zhang guan \\
\hline 21 & Fujian Province & Longyan City & NA & 25.161 & 116.933 & Anthracite & Small & Keng bing \\
\hline 22 & Fujian Province & Longyan City & NA & 25.015 & 116.968 & Anthracite & Small & Ding feng shan \\
\hline 23 & Fujian Province & Longyan City & NA & 25.069 & 116.972 & Anthracite & Small & Hu keng shan \\
\hline 24 & Fujian Province & Longyan City & $\mathrm{NA}$ & 25.062 & 117.005 & Anthracite & Small & Hong tan shan \\
\hline 25 & Fujian Province & Longyan City & NA & 25.189 & 116.991 & Anthracite & Small & Jiang shan \\
\hline 26 & Fujian Province & Longyan City & NA & 25.052 & 117.069 & Anthracite & Small & Wang Pit \\
\hline 27 & Fujian Province & Longyan City & NA & 25.091 & 117.054 & Anthracite & Small & Cui ping shan \\
\hline 28 & Fujian Province & Longyan City & $\mathrm{NA}$ & 25.076 & 117.070 & Anthracite & Small & Huang Pit \\
\hline 29 & Fujian Province & Longyan City & $\mathrm{NA}$ & 25.027 & 117.091 & Anthracite & Small & Chen Pit \\
\hline 30 & Fujian Province & Longyan City & NA & 25.149 & 117.098 & Anthracite & Small & Lu jia de \\
\hline 31 & Fujian Province & Longyan City & NA & 25.177 & 117.097 & Anthracite & Small & Jin tou \\
\hline 32 & Fujian Province & Longyan City & NA & 25.106 & 117.106 & Anthracite & Small & Xie jia bang \\
\hline 33 & Fujian Province & Longyan City & $\mathrm{NA}$ & 25.138 & 117.112 & Anthracite & Small & Nei luo qiao \\
\hline 34 & Fujian Province & Longyan City & $\mathrm{NA}$ & 25.224 & 117.131 & Anthracite & Small & Feng shan qi \\
\hline 35 & Fujian Province & Longyan City & NA & 25.281 & 117.176 & Anthracite & Small & Chi Pit \\
\hline 36 & Fujian Province & Longyan City & $\mathrm{NA}$ & 25.323 & 117.178 & Anthracite & Small & Su bang \\
\hline 37 & Fujian Province & Longyan City & NA & 25.388 & 117.213 & Anthracite & Small & Bai sha \\
\hline 38 & Fujian Province & Sanming City & Yongan City & 25.985 & 117.204 & Anthracite & Small & Jia fu \\
\hline
\end{tabular}


Table 1. List of coal mines in China.-Continued

[Abbreviations used: dec. deg. - decimal degrees; NA - not available or not applicable]

\begin{tabular}{|c|c|c|c|c|c|c|c|c|}
\hline $\begin{array}{c}\text { ID } \\
\text { Number }\end{array}$ & $\begin{array}{c}\text { Province, National } \\
\text { Municipality, or } \\
\text { Autonomous Region }\end{array}$ & $\begin{array}{c}\text { Prefecture, Municipality, } \\
\text { Prefecture-Level City, or } \\
\text { League }\end{array}$ & $\begin{array}{l}\text { County, District or } \\
\text { County-Level City }\end{array}$ & $\begin{array}{l}\text { Latitude } \\
\text { (dec. deg.) }\end{array}$ & $\begin{array}{l}\text { Longitude } \\
\text { (dec. deg.) }\end{array}$ & Rank & $\begin{array}{l}\text { Relative } \\
\text { Mine Size }\end{array}$ & Mine Name \\
\hline 39 & Fujian Province & Sanming City & Yongan City & 25.881 & 117.243 & Anthracite & Small & Hong tian \\
\hline 40 & Fujian Province & Longyan City & NA & 25.296 & 117.272 & Bituminous & Small & Su ban \\
\hline 41 & Fujian Province & Sanming City & Yongan City & 25.988 & 117.326 & Anthracite & Small & Xia ha \\
\hline 42 & Fujian Province & Longyan City & Zhangping City & 25.513 & 117.419 & Bituminous & Small & Tai hang \\
\hline 43 & Fujian Province & Longyan City & Zhangping City & 25.365 & 117.452 & Bituminous & Small & An jing \\
\hline 44 & Fujian Province & Longyan City & Zhangping City & 25.615 & 117.622 & Anthracite & Small & Qi tou shan \\
\hline 45 & Fujian Province & Sanming City & Datian County & 25.746 & 117.708 & Anthracite & Small & Shang jing \\
\hline 46 & Fujian Province & Quanzhou City & Anxi County & 25.332 & 117.942 & Anthracite & Small & Jian dou \\
\hline 47 & Fujian Province & Quanzhou City & Yongchun County & 25.474 & 117.979 & Anthracite & Small & Tian hu shan \\
\hline 48 & Fujian Province & Quanzhou City & Dehua County & 25.569 & 118.055 & Anthracite & Small & Yang shan \\
\hline 49 & Fujian Province & Quanzhou City & Dehua County & 25.812 & 118.216 & Anthracite & Small & Ceng ban \\
\hline 50 & Fujian Province & Longyan City & Wuping County & 24.954 & 116.250 & Anthracite & Small & Ning yang \\
\hline 51 & Fujian Province & Longyan City & Yongding County & 24.952 & 116.909 & Anthracite & Small & Fu ling \\
\hline 52 & Fujian Province & Longyan City & Yongding County & 24.905 & 116.919 & Anthracite & Small & Wa yao ping \\
\hline 53 & Fujian Province & Longyan City & Yongding County & 24.928 & 116.954 & Anthracite & Small & Kong fu \\
\hline 54 & Fujian Province & Longyan City & Yongding County & 24.953 & 116.962 & Anthracite & Small & Pui feng \\
\hline 55 & Fujian Province & Longyan City & Yongding County & 24.894 & 116.979 & Anthracite & Small & Dong zhong \\
\hline 56 & Fujian Province & Longyan City & Yongding County & 24.876 & 117.036 & Anthracite & Small & Long tan \\
\hline 57 & Fujian Province & Longyan City & Yongding County & 24.834 & 117.051 & Anthracite & Small & Zhang ta \\
\hline 1 & Gansu Province & Jiuquan City & $\begin{array}{l}\text { Subei Autonomous } \\
\text { County }\end{array}$ & 41.547 & 96.730 & Bituminous & Small & Ma zong shan \\
\hline 2 & Gansu Province & Jiuquan City & Jinta County & 40.682 & 99.169 & Bituminous & Small & Ji ji tai zi \\
\hline 3 & Gansu Province & Jiuquan City & Yumen County & 39.843 & 97.066 & Bituminous & Small & Dong da yao \\
\hline 4 & Gansu Province & Zhangye City & $\begin{array}{l}\text { Sunan Autonomous } \\
\text { County }\end{array}$ & 39.747 & 97.265 & Bituminous & Small & Liu shu gou \\
\hline 6 & Gansu Province & Zhangye City & $\begin{array}{l}\text { Sunan Autonomous } \\
\text { County }\end{array}$ & 39.113 & 99.767 & Bituminous & Small & Yao gou \\
\hline 7 & Gansu Province & Zhangye City & $\begin{array}{l}\text { Sunan Autonomous } \\
\text { County }\end{array}$ & 38.961 & 99.799 & Anthracite & Small & Zao ji mou \\
\hline 8 & Gansu Province & Zhangye City & $\begin{array}{l}\text { Sunan Autonomous } \\
\text { County }\end{array}$ & 38.942 & 99.881 & Bituminous & Small & Da xiao le ba \\
\hline 9 & Gansu Province & Zhangye City & $\begin{array}{l}\text { Sunan Autonomous } \\
\text { County }\end{array}$ & 38.898 & 99.715 & Bituminous & Small & Hui da ban \\
\hline 10 & Gansu Province & Zhangye City & $\begin{array}{l}\text { Sunan Autonomous } \\
\text { County }\end{array}$ & 38.781 & 99.971 & Bituminous & Small & Kanglong si \\
\hline 11 & Gansu Province & Zhangye City & Linze County & 39.667 & 100.118 & Bituminous & Small & Lu quan shan \\
\hline
\end{tabular}


Table 1. List of coal mines in China. - Continued

[Abbreviations used: dec. deg. - decimal degrees; NA - not available or not applicable]

\begin{tabular}{|c|c|c|c|c|c|c|c|c|}
\hline $\begin{array}{c}\text { ID } \\
\text { Number }\end{array}$ & $\begin{array}{c}\text { Province, National } \\
\text { Municipality, or } \\
\text { Autonomous Region }\end{array}$ & $\begin{array}{c}\text { Prefecture, Municipality, } \\
\text { Prefecture-Level City, or } \\
\text { League }\end{array}$ & $\begin{array}{l}\text { County, District or } \\
\text { County-Level City }\end{array}$ & $\begin{array}{l}\text { Latitude } \\
\text { (dec. deg.) }\end{array}$ & $\begin{array}{l}\text { Longitude } \\
\text { (dec. deg.) }\end{array}$ & Rank & $\begin{array}{l}\text { Relative } \\
\text { Mine Size }\end{array}$ & Mine Name \\
\hline 12 & Gansu Province & Zhangye City & $\begin{array}{l}\text { Sunan Autonomous } \\
\text { County }\end{array}$ & 38.676 & 100.248 & Bituminous & Small & Yao cao wa \\
\hline 13 & Gansu Province & Zhangye City & Shandan County & 38.680 & 101.000 & Bituminous & Small & Ping po \\
\hline 14 & Gansu Province & Zhangye City & Shandan County & 38.652 & 101.296 & Bituminous & Small & Xin he \\
\hline 15 & Gansu Province & Zhangye City & Shandan County & 38.544 & 101.465 & Bituminous & Small & Dong shui quan \\
\hline 16 & Gansu Province & Zhangye City & $\begin{array}{l}\text { Sunan Autonomous } \\
\text { County }\end{array}$ & 38.537 & 100.266 & Bituminous & Small & Da ye kou guan tai \\
\hline 17 & Gansu Province & Zhangye City & $\begin{array}{l}\text { Sunan Autonomous } \\
\text { County }\end{array}$ & 38.490 & 100.378 & Bituminous & Small & $\mathrm{Su}$ qu kou \\
\hline 18 & Gansu Province & Zhangye City & Shandan County & 38.444 & 101.441 & Bituminous & Small & Yang hu gou \\
\hline 19 & Gansu Province & Jinchang City & Yongchang County & 38.336 & 101.922 & Bituminous & Small & Mao bo la \\
\hline 20 & Gansu Province & Jinchang City & Yongchang County & 38.302 & 101.614 & Bituminous & Small & Hong shan yao \\
\hline 21 & Gansu Province & Zhangye City & Minle County & 38.270 & 100.786 & Bituminous & Small & Man shan yao \\
\hline 22 & Gansu Province & Zhangye City & Shandan County & 38.227 & 101.369 & Bituminous & Small & $\begin{array}{l}\text { Da ma ying. zhang } \\
\text { shan zi }\end{array}$ \\
\hline 23 & Gansu Province & Jinchang City & Yongchang County & 38.191 & 101.728 & Anthracite & Small & Miao er gou \\
\hline 24 & Gansu Province & Jinchang City & Yongchang County & 38.079 & 101.592 & Bituminous & Small & Ma ying gou \\
\hline 25 & Gansu Province & Jinchang City & Yongchang County & 38.301 & 102.032 & Bituminous & Small & Ma jia w \\
\hline 26 & Gansu Province & Wuwei City & NA & 38.036 & 102.210 & Bituminous & Small & Da kou zi \\
\hline 27 & Gansu Province & Zhangye City & $\begin{array}{l}\text { Sunan Autonomous } \\
\text { County }\end{array}$ & 37.908 & 101.945 & Anthracite & Small & Bing gou \\
\hline 28 & Gansu Province & Zhangye City & $\begin{array}{l}\text { Sunan Autonomous } \\
\text { County }\end{array}$ & 37.876 & 102.052 & Anthracite & Small & Jiu tiao ling \\
\hline 29 & Gansu Province & Wuwei City & Gulang County & 37.595 & 103.346 & Bituminous & Small & Gu shan dun \\
\hline 30 & Gansu Province & Baiyin City & Jingtai County & 37.483 & 103.963 & Bituminous & Small & $\begin{array}{c}\text { Fang jia Well - New } \\
\text { Western Well }\end{array}$ \\
\hline 31 & Gansu Province & Wuwei City & Gulang County & 37.490 & 103.748 & Bituminous & Small & Da quan shui \\
\hline 32 & Gansu Province & Wuwei City & $\begin{array}{l}\text { Tianzhu Autonomous } \\
\text { County }\end{array}$ & 37.187 & 103.366 & Bituminous & Small & Da e ba \\
\hline 33 & Gansu Province & Baiyin City & Jingtai County & 36.976 & 103.936 & Bituminous & Small & Da la pai \\
\hline 34 & Gansu Province & Wuwei City & $\begin{array}{l}\text { Tianzhu Autonomous } \\
\text { County }\end{array}$ & 36.968 & 102.658 & Bituminous & Small & Tan shan ling \\
\hline 36 & Gansu Province & Wuwei City & $\begin{array}{l}\text { Tianzhu Autonomous } \\
\text { County }\end{array}$ & 36.810 & 102.820 & Lignite & Small & Da tan \\
\hline 37 & Gansu Province & Lanzhou City & Yongdeng County & 36.794 & 102.908 & Lignite & Small & Da you \\
\hline 38 & Gansu Province & Lanzhou City & NA & 36.416 & 102.846 & Bituminous & Small & Hong gu qu yao jie \\
\hline
\end{tabular}


Table 1. List of coal mines in China.-Continued

[Abbreviations used: dec. deg. - decimal degrees; NA - not available or not applicable]

\begin{tabular}{|c|c|c|c|c|c|c|c|c|}
\hline $\begin{array}{c}\text { ID } \\
\text { Number }\end{array}$ & $\begin{array}{l}\text { Province, National } \\
\text { Municipality, or } \\
\text { Autonomous Region }\end{array}$ & $\begin{array}{c}\text { Prefecture, Municipality, } \\
\text { Prefecture-Level City, or } \\
\text { League }\end{array}$ & $\begin{array}{l}\text { County, District or } \\
\text { County-Level City }\end{array}$ & $\begin{array}{l}\text { Latitude } \\
\text { (dec. deg.) }\end{array}$ & $\begin{array}{l}\text { Longitude } \\
\text { (dec. deg.) }\end{array}$ & Rank & $\begin{array}{l}\text { Relative } \\
\text { Mine Size }\end{array}$ & Mine Name \\
\hline 40 & Gansu Province & Baiyin City & Jingtai County & 37.393 & 104.608 & Anthracite & Small & $\begin{array}{l}\text { Ku shui gou, hong } \\
\text { shan }\end{array}$ \\
\hline 41 & Gansu Province & Baiyin City & Jingtai County & 37.122 & 104.223 & Bituminous & Small & Xiang shui \\
\hline 42 & Gansu Province & Baiyin City & Jingyuan County & 36.960 & 104.732 & Bituminous & Small & Jing er chuan \\
\hline 43 & Gansu Province & Baiyin City & Jingyuan County & 36.898 & 104.827 & Bituminous & Small & Wang jia shan \\
\hline 44 & Gansu Province & Baiyin City & Jingyuan County & 36.739 & 104.911 & Bituminous & Small & Bao ji shan \\
\hline 45 & Gansu Province & Baiyin City & Jingyuan County & 36.739 & 105.096 & Bituminous & Small & Coal Pit Pond \\
\hline 46 & Gansu Province & Baiyin City & Jingyuan County & 36.685 & 104.952 & Bituminous & Small & Hong qi shan \\
\hline 47 & Gansu Province & Baiyin City & Jingyuan County & 36.680 & 105.034 & Bituminous & Small & Hong hui \\
\hline 48 & Gansu Province & Qingyang City & Huan County & 37.068 & 106.914 & Bituminous & Small & Tian shui bao \\
\hline 49 & Gansu Province & Qingyang City & Huan County & 37.066 & 106.790 & Bituminous & Small & $\begin{array}{l}\text { Tian shui bao } \\
\text { Southern Part }\end{array}$ \\
\hline 50 & Gansu Province & Lanzhou City & NA & 35.913 & 103.841 & Bituminous & Small & Qi li he, A gan zhen \\
\hline 51 & Gansu Province & Lanzhou City & Yuzhong County & 35.911 & 103.953 & Bituminous & Small & Shui cha gou \\
\hline 52 & Gansu Province & Gannan City & Luqu County & 34.189 & 102.599 & Bituminous & Small & Ga hai \\
\hline 53 & Gansu Province & Baiyin City & Huining County & 35.607 & 105.499 & Anthracite & Small & Shang jie guan zi xia \\
\hline 54 & Gansu Province & Pingliang City & NA & 35.409 & 106.674 & Bituminous & Small & Nos. $2 \& 3$ Daogou \\
\hline 55 & Gansu Province & Pingliang City & NA & 35.373 & 106.731 & Bituminous & Small & Xiao wan zi \\
\hline 56 & Gansu Province & Pingliang City & NA & 35.292 & 106.833 & Bituminous & Small & Tu gu dui \\
\hline 57 & Gansu Province & Pingliang City & Huating County & 35.259 & 106.734 & Bituminous & Large & Hua ting \\
\hline 60 & Gansu Province & Pingliang City & Chongxin County & 35.200 & 106.977 & Bituminous & Small & Chi cheng \\
\hline 61 & Gansu Province & Pingliang City & Chongxin County & 35.166 & 106.945 & Bituminous & Large & An kou - xin yao \\
\hline 62 & Gansu Province & Qingyang City & Zhengning County & 35.430 & 108.264 & Bituminous & Large & Southern Part \\
\hline 63 & Gansu Province & Gannan City & Huixian County & 33.903 & 105.850 & Bituminous & Small & Guan an shan \\
\hline 64 & Gansu Province & Gannan City & Zhouqu County & 33.608 & 104.371 & Bituminous & Small & Wu ping \\
\hline 65 & Gansu Province & Longnan City & Wudu County & 33.595 & 104.838 & Bituminous & Small & Long jia gou \\
\hline 66 & Gansu Province & Gannan City & Liangdang County & 33.778 & 106.397 & Bituminous & Small & $\mathrm{Xi}$ pi \\
\hline 1 & Guangdong Province & Qingyang City & Lianzhou City & 25.067 & 112.630 & Anthracite & Small & Da lu bian \\
\hline 2 & Guangdong Province & Shaoguan City & Lechang City & 25.348 & 113.111 & Bituminous & Small & Luo jia du \\
\hline 3 & Guangdong Province & Shaoguan City & Lechang City & 25.270 & 113.052 & Bituminous & Small & Han chong \\
\hline 4 & Guangdong Province & Shaoguan City & Lechang City & 25.229 & 113.022 & Bituminous & Small & Guan chun \\
\hline 5 & Guangdong Province & Shaoguan City & Renhua County & 25.097 & 113.669 & Anthracite & Small & Ma tang \\
\hline 6 & Guangdong Province & Shaoguan City & Renhua County & 25.055 & 113.582 & Anthracite & Small & Ge ding zhai \\
\hline 7 & Guangdong Province & Shaoguan City & Lechang City & 25.019 & 113.426 & Bituminous & Small & An kou \\
\hline
\end{tabular}


Table 1. List of coal mines in China. - Continued

[Abbreviations used: dec. deg. - decimal degrees; NA - not available or not applicable]

\begin{tabular}{|c|c|c|c|c|c|c|c|c|}
\hline $\begin{array}{c}\text { ID } \\
\text { Number }\end{array}$ & $\begin{array}{l}\text { Province, National } \\
\text { Municipality, or } \\
\text { Autonomous Region }\end{array}$ & $\begin{array}{c}\text { Prefecture, Municipality, } \\
\text { Prefecture-Level City, or } \\
\text { League }\end{array}$ & $\begin{array}{l}\text { County, District or } \\
\text { County-Level City }\end{array}$ & $\begin{array}{l}\text { Latitude } \\
\text { (dec. deg.) }\end{array}$ & $\begin{array}{l}\text { Longitude } \\
\text { (dec. deg.) }\end{array}$ & Rank & $\begin{array}{l}\text { Relative } \\
\text { Mine Size }\end{array}$ & Mine Name \\
\hline 8 & Guangdong Province & Qingyuan City & Lianzhou City & 24.922 & 112.362 & Anthracite & Small & Bao an \\
\hline 9 & Guangdong Province & Qingyuan City & Lianzhou City & 24.877 & 112.436 & Anthracite & Small & San kou jiang \\
\hline 10 & Guangdong Province & Qingyuan City & Lianzhou City & 24.805 & 112.311 & Anthracite & Small & Li yu kou \\
\hline 11 & Guangdong Province & Qingyuan City & Lianzhou City & 24.759 & 112.497 & Anthracite & Small & Da mai kong \\
\hline 12 & Guangdong Province & Qingyuan City & Yangshan County & 24.747 & 112.664 & Anthracite & Small & Ma xia \\
\hline 13 & Guangdong Province & Qingyuan City & Lianzhou City & 24.746 & 112.349 & Anthracite & Small & Che tian \\
\hline 14 & Guangdong Province & Qingyuan City & Lianzhou City & 24.681 & 112.354 & Anthracite & Small & Jiu po \\
\hline 15 & Guangdong Province & Qingyuan City & Lianzhou City & 24.648 & 112.364 & Bituminous & Small & San y kou \\
\hline 16 & Guangdong Province & Qingyuan City & $\begin{array}{l}\text { Liannan Autonomous } \\
\text { County }\end{array}$ & 24.600 & 112.344 & Bituminous & Small & Ma mu zhai \\
\hline 17 & Guangdong Province & Qingyuan City & Yangshan County & 24.629 & 112.620 & Anthracite & Small & Shuang shan \\
\hline 18 & Guangdong Province & Shaoguan City & Qujiang District & 25.002 & 113.553 & Anthracite & Small & Hua ping \\
\hline 19 & Guangdong Province & Shaoguan City & NA & 24.951 & 113.447 & Anthracite & Small & Xian ren ying \\
\hline 20 & Guangdong Province & Shaoguan City & Qujiang District & 24.956 & 113.568 & Anthracite & Small & Cha shan \\
\hline 21 & Guangdong Province & Shaoguan City & Qujiang District & 24.935 & 113.610 & Anthracite & Small & Zhe gu shi Mine \\
\hline 22 & Guangdong Province & Shaoguan City & Qujiang District & 24.836 & 113.642 & Anthracite & Small & Hua la zhai \\
\hline 23 & Guangdong Province & Shaoguan City & NA & 24.798 & 113.551 & Anthracite & Small & Fu rong shan \\
\hline 24 & Guangdong Province & Shaoguan City & Qujiang District & 24.811 & 113.734 & Anthracite & Small & Jiang jun ling \\
\hline 25 & Guangdong Province & Shaoguan City & Qujiang District & 24.779 & 113.813 & Anthracite & Small & Zao tian \\
\hline 26 & Guangdong Province & Shaoguan City & Qujiang District & 24.766 & 113.432 & Bituminous & Small & Hong wei Pit \\
\hline 27 & Guangdong Province & Shaoguan City & Qujiang District & 24.700 & 113.419 & Anthracite & Small & An cun \\
\hline 29 & Guangdong Province & Qingyuan City & Yingde City & 24.296 & 113.831 & Anthracite & Small & Liao wu shan \\
\hline 30 & Guangdong Province & Qingyuan City & Yingde City & 24.212 & 113.877 & Anthracite & Small & Qing tang \\
\hline 31 & Guangdong Province & Shaoguan City & Shixing City & 24.925 & 114.003 & Anthracite & Small & Wu shi wei \\
\hline 32 & Guangdong Province & Shaoguan City & Shixing City & 24.908 & 114.090 & Anthracite & Small & Nan shan \\
\hline 33 & Guangdong Province & Shaoguan City & Wengyuan County & 24.502 & 114.117 & Anthracite & Small & San Pit \\
\hline 34 & Guangdong Province & Shaoguan City & Wengyuan County & 24.455 & 114.128 & Anthracite & Small & Jiang ba \\
\hline 35 & Guangdong Province & Heyuan City & Lianping County & 24.450 & 114.283 & Anthracite & Small & $\mathrm{Zi} \mathrm{xi}$ \\
\hline 36 & Guangdong Province & Shaoguan City & Wengyuan County & 24.382 & 114.211 & Anthracite & Small & Feng shan \\
\hline 37 & Guangdong Province & Heyuan City & Lianping County & 24.381 & 114.503 & Anthracite & Small & Hong shan \\
\hline 38 & Guangdong Province & Shaoguan City & Wengyuan County & 24.296 & 114.097 & Anthracite & Small & Kun shan \\
\hline 39 & Guangdong Province & Heyuan City & Lianping County & 24.253 & 114.675 & Anthracite & Small & Xin xi \\
\hline 40 & Guangdong Province & Heyuan City & Lianping County & 24.214 & 114.721 & Anthracite & Small & Jing kou \\
\hline 41 & Guangdong Province & Heyuan City & Lianping County & 24.258 & 114.817 & Anthracite & Small & Da hu \\
\hline
\end{tabular}


Table 1. List of coal mines in China. - Continued

[Abbreviations used: dec. deg. - decimal degrees; NA - not available or not applicable]

\begin{tabular}{|c|c|c|c|c|c|c|c|c|}
\hline $\begin{array}{c}\text { ID } \\
\text { Number }\end{array}$ & $\begin{array}{c}\text { Province, National } \\
\text { Municipality, or } \\
\text { Autonomous Region }\end{array}$ & $\begin{array}{c}\text { Prefecture, Municipality, } \\
\text { Prefecture-Level City, or } \\
\text { League }\end{array}$ & $\begin{array}{l}\text { County, District or } \\
\text { County-Level City }\end{array}$ & $\begin{array}{l}\text { Latitude } \\
\text { (dec. deg.) }\end{array}$ & $\begin{array}{l}\text { Longitude } \\
\text { (dec. deg.) }\end{array}$ & Rank & $\begin{array}{l}\text { Relative } \\
\text { Mine Size }\end{array}$ & Mine Name \\
\hline 42 & Guangdong Province & Shaoguan City & Xinfeng County & 24.143 & 114.408 & Anthracite & Small & Zhang tian Pit \\
\hline 43 & Guangdong Province & Shaoguan City & Xinfeng County & 24.495 & 115.789 & Anthracite & Small & Si wang zhang \\
\hline 44 & Guangdong Province & Shaoguan City & Xinfeng County & 24.473 & 115.753 & Anthracite & Small & Li shu Pit \\
\hline 45 & Guangdong Province & Shaoguan City & Xinfeng County & 24.462 & 115.830 & Anthracite & Small & Long hu \\
\hline 46 & Guangdong Province & Shaoguan City & Xinfeng County & 24.434 & 115.761 & Anthracite & Small & Nan shan xia \\
\hline 47 & Guangdong Province & Meizhou City & Mei County & 24.426 & 115.919 & Anthracite & Small & Zhu Pit \\
\hline 48 & Guangdong Province & Shaoguan City & Xinfeng County & 24.406 & 115.648 & Anthracite & Small & Gan cun \\
\hline 49 & Guangdong Province & Shaoguan City & Xinfeng County & 24.349 & 115.686 & Anthracite & Small & Ping yang \\
\hline 50 & Guangdong Province & Meizhou City & Mei County & 24.346 & 115.971 & Anthracite & Small & Da ping \\
\hline 51 & Guangdong Province & Meizhou City & Wuhua County & 24.007 & 115.474 & Anthracite & Small & Huang ta \\
\hline 52 & Guangdong Province & Meizhou City & Mei County & 24.681 & 116.447 & Anthracite & Small & Hong kui Pit \\
\hline 53 & Guangdong Province & Meizhou City & Mei County & 24.639 & 116.399 & Anthracite & Small & Bao Pit \\
\hline 54 & Guangdong Province & Meizhou City & Jiaoling County & 24.580 & 116.233 & Anthracite & Small & Da di \\
\hline 55 & Guangdong Province & Meizhou City & Mei County & 24.466 & 116.158 & Anthracite & Small & Bai du \\
\hline 56 & Guangdong Province & Meizhou City & Mei County & 24.380 & 116.128 & Anthracite & Small & Heng jing \\
\hline 57 & Guangdong Province & Meizhou City & Mei County & 24.391 & 116.223 & Anthracite & Small & Xie tian \\
\hline 58 & Guangdong Province & Meizhou City & Mei County & 24.370 & 116.288 & Anthracite & Small & Cheng zi ji \\
\hline 59 & Guangdong Province & Meizhou City & Mei County & 24.272 & 116.012 & Anthracite & Small & Hua ig gang \\
\hline 60 & Guangdong Province & Meizhou City & Mei County & 24.272 & 116.335 & Anthracite & Small & Ming shan zhang \\
\hline 62 & Guangdong Province & Zhaoqing City & Sihui City & 23.605 & 112.701 & Anthracite & Small & San gui \\
\hline 64 & Guangdong Province & Zhaoqing City & Gaoyao City & 23.194 & 112.280 & Anthracite & Small & $\mathrm{Lu} \mathrm{bu}$ \\
\hline 71 & Guangdong Province & Zhaoqing City & Gaoyao City & 23.091 & 112.776 & Anthracite & Small & Jin li \\
\hline 72 & Guangdong Province & Zhaoqing City & Gaoyao City & 23.034 & 112.667 & Anthracite & Small & Xian gang \\
\hline 73 & Guangdong Province & Zhaoqing City & Gaoyao City & 23.030 & 112.756 & Anthracite & Small & Luo ke \\
\hline 74 & Guangdong Province & Foshan City & Sanshui District & 23.021 & 112.858 & Bituminous & Small & $\mathrm{Da}$ \\
\hline 76 & Guangdong Province & Foshan City & Nanhai District & 23.272 & 113.093 & Bituminous & Small & Wen tou ling \\
\hline 77 & Guangdong Province & Foshan City & Nanhai District & 23.217 & 113.102 & Anthracite & Small & Yao ling \\
\hline 78 & Guangdong Province & Guangzhou City & NA & 23.203 & 113.263 & Anthracite & Small & Jia he \\
\hline 79 & Guangdong Province & Foshan City & Nanhai District & 23.133 & 113.097 & Anthracite & Small & Lian biao \\
\hline 81 & Guangdong Province & Huizhou City & Longmen County & 23.675 & 114.322 & Anthracite & Small & Ping ling \\
\hline 82 & Guangdong Province & Huizhou City & Longmen County & 23.587 & 114.115 & Anthracite & Small & Shuang dong \\
\hline 83 & Guangdong Province & Huizhou City & Longmen County & 23.554 & 114.282 & Anthracite & Small & Lu xi \\
\hline 84 & Guangdong Province & Huizhou City & Boluo County & 23.359 & 114.487 & Anthracite & Small & Xiu ling \\
\hline 85 & Guangdong Province & Huizhou City & Boluo County & 23.199 & 114.548 & Anthracite & Small & Tie ye pai \\
\hline
\end{tabular}


Table 1. List of coal mines in China. - Continued

[Abbreviations used: dec. deg. - decimal degrees; NA - not available or not applicable]

\begin{tabular}{|c|c|c|c|c|c|c|c|c|}
\hline $\begin{array}{c}\text { ID } \\
\text { Number }\end{array}$ & $\begin{array}{c}\text { Province, National } \\
\text { Municipality, or } \\
\text { Autonomous Region }\end{array}$ & $\begin{array}{c}\text { Prefecture, Municipality, } \\
\text { Prefecture-Level City, or } \\
\text { League }\end{array}$ & $\begin{array}{l}\text { County, District or } \\
\text { County-Level City }\end{array}$ & $\begin{array}{l}\text { Latitude } \\
\text { (dec. deg.) }\end{array}$ & $\begin{array}{l}\text { Longitude } \\
\text { (dec. deg.) }\end{array}$ & Rank & $\begin{array}{l}\text { Relative } \\
\text { Mine Size }\end{array}$ & Mine Name \\
\hline 89 & Guangdong Province & Huizhou City & Zijin County & 23.455 & 115.092 & Anthracite & Small & Jiu he \\
\hline 90 & Guangdong Province & Jieyang City & Huilai County & 23.096 & 115.993 & Anthracite & Small & Nan chi du \\
\hline 94 & Guangdong Province & Jieyang City & Puning City & 23.109 & 116.029 & Anthracite & Small & Ma an shan \\
\hline 96 & Guangdong Province & Yunfu City & NA & 22.715 & 111.910 & Anthracite & Small & Xiao yun wu shan \\
\hline 98 & Guangdong Province & Yangjiang City & Yangchun City & 22.461 & 111.918 & Bituminous & Small & Long y gang \\
\hline 99 & Guangdong Province & Yangjiang City & Yangchun City & 22.432 & 111.919 & Bituminous & Small & Xian ji Pit \\
\hline 100 & Guangdong Province & Yangjiang City & Yangchun City & 22.383 & 111.903 & Bituminous & Small & Tan liao \\
\hline 101 & Guangdong Province & Yangjiang City & Yangchun City & 22.353 & 111.869 & Anthracite & Small & Zhu lon gao \\
\hline 102 & Guangdong Province & Yangjiang City & Yangchun City & 22.187 & 111.754 & Anthracite & Small & Kong dong \\
\hline 103 & Guangdong Province & Zhaoqing City & Gaoyao City & 22.984 & 112.380 & Anthracite & Small & Ma an \\
\hline 106 & Guangdong Province & Foshan City & Gaoming District & 22.942 & 112.753 & Anthracite & Small & He cun \\
\hline 109 & Guangdong Province & Foshan City & Gaoming District & 22.882 & 112.702 & Anthracite & Small & Guan jing \\
\hline 116 & Guangdong Province & Jiangmen City & Enping City & 22.362 & 112.453 & Anthracite & Small & Jin sha \\
\hline 118 & Guangdong Province & Jiangmen City & Taishan City & 22.283 & 112.597 & Anthracite & Small & Bai sha \\
\hline 119 & Guangdong Province & Jiangmen City & Kaiping City & 22.188 & 112.495 & Anthracite & Small & Jin ji \\
\hline 132 & Guangdong Province & Maoming City & Gaozhou City & 21.806 & 110.788 & Lignite & Large & Shi gu \\
\hline 133 & Guangdong Province & Maoming City & NA & 21.739 & 110.844 & Lignite & Medium & Jing tang \\
\hline 134 & Guangdong Province & Maoming City & Dianbai County & 21.671 & 110.951 & Lignite & Medium & Yang jiao \\
\hline 4 & $\begin{array}{l}\text { Guangxi Zhuang } \\
\text { Autonomous Region }\end{array}$ & Hechi City & $\begin{array}{l}\text { Huanjiang Autonomous } \\
\text { County }\end{array}$ & 25.378 & 108.358 & Anthracite & Small & Hongshan \\
\hline 65 & $\begin{array}{l}\text { Guangxi Zhuang } \\
\text { Autonomous Region }\end{array}$ & Hechi City & Luocheng County & 24.803 & 108.844 & Anthracite & Small & Luo cheng \\
\hline 71 & $\begin{array}{l}\text { Guangxi Zhuang } \\
\text { Autonomous Region }\end{array}$ & Hechi City & Yizhou City & 24.562 & 108.346 & Bituminous & Small & Yi shan \\
\hline 73 & $\begin{array}{l}\text { Guangxi Zhuang } \\
\text { Autonomous Region }\end{array}$ & Hechi City & $\begin{array}{l}\text { Du'an Autonomous } \\
\text { County }\end{array}$ & 24.190 & 108.348 & Bituminous & Small & Bai wang \\
\hline 102 & $\begin{array}{l}\text { Guangxi Zhuang } \\
\text { Autonomous Region }\end{array}$ & Zhongshan & NA & 24.450 & 111.442 & Bituminous & Small & $\mathrm{Xi}$ wan \\
\hline 105 & $\begin{array}{l}\text { Guangxi Zhuang } \\
\text { Autonomous Region }\end{array}$ & Baise City & NA & 23.854 & 106.621 & Bituminous & Medium & $\begin{array}{l}\text { Baise Coal Field, East } \\
\text { sun, Gong lou, } \\
\text { East huai }\end{array}$ \\
\hline 138 & $\begin{array}{l}\text { Guangxi Zhuang } \\
\text { Autonomous Region }\end{array}$ & Laibin City & Heshan City & 23.827 & 108.943 & Bituminous & Medium & He shan \\
\hline 140 & $\begin{array}{l}\text { Guangxi Zhuang } \\
\text { Autonomous Region }\end{array}$ & Nanning City & Shanlin County & 23.749 & 108.631 & Bituminous & Small & Qiao an \\
\hline
\end{tabular}


Table 1. List of coal mines in China. - Continued

[Abbreviations used: dec. deg. - decimal degrees; NA - not available or not applicable]

\begin{tabular}{|c|c|c|c|c|c|c|c|c|}
\hline $\begin{array}{c}\text { ID } \\
\text { Number }\end{array}$ & $\begin{array}{l}\text { Province, National } \\
\text { Municipality, or } \\
\text { Autonomous Region }\end{array}$ & $\begin{array}{c}\text { Prefecture, Municipality, } \\
\text { Prefecture-Level City, or } \\
\text { League }\end{array}$ & $\begin{array}{l}\text { County, District or } \\
\text { County-Level City }\end{array}$ & $\begin{array}{l}\text { Latitude } \\
\text { (dec. deg.) }\end{array}$ & $\begin{array}{l}\text { Longitude } \\
\text { (dec. deg.) }\end{array}$ & Rank & $\begin{array}{l}\text { Relative } \\
\text { Mine Size }\end{array}$ & Mine Name \\
\hline 175 & $\begin{array}{l}\text { Guangxi Zhuang } \\
\text { Autonomous Region }\end{array}$ & Chongzuo City & Fusui County & 22.412 & 107.618 & Bituminous & Medium & Fu sui \\
\hline 177 & $\begin{array}{l}\text { Guangxi Zhuang } \\
\text { Autonomous Region }\end{array}$ & Chongzuo City & Ningming County & 22.141 & 107.415 & Lignite & Small & Hai yuan \\
\hline 178 & $\begin{array}{l}\text { Guangxi Zhuang } \\
\text { Autonomous Region }\end{array}$ & Fangchenggang City & Shangsi County & 22.132 & 107.802 & Lignite & Medium & Shang si \\
\hline 180 & $\begin{array}{l}\text { Guangxi Zhuang } \\
\text { Autonomous Region }\end{array}$ & Nanning City & NA & 22.955 & 108.032 & Lignite & Medium & Outskirts of $\mathrm{Na}$ long \\
\hline 183 & $\begin{array}{l}\text { Guangxi Zhuang } \\
\text { Autonomous Region }\end{array}$ & Nanning City & NA & 22.813 & 108.230 & Lignite & Small & Nan ning \\
\hline 188 & $\begin{array}{l}\text { Guangxi Zhuang } \\
\text { Autonomous Region }\end{array}$ & Qinzhou City & NA & 22.110 & 108.620 & Bituminous & Small & Ren zi ping \\
\hline 1 & Guizhou Province & Zunyi City & Tongzi County & 28.672 & 106.901 & Bituminous & Small & Mu gua \\
\hline 2 & Guizhou Province & Zunyi City & Tongzi County & 28.437 & 106.947 & Bituminous & Small & Qian jin ba \\
\hline 3 & Guizhou Province & Zunyi City & Xishui County & 28.273 & 106.174 & Anthracite & Small & Ma lin \\
\hline 4 & Guizhou Province & Zunyi City & Xishui County & 28.312 & 106.275 & Anthracite & Small & Bai ni \\
\hline 5 & Guizhou Province & Zunyi City & Tongzi County & 28.335 & 106.841 & Bituminous & Small & Da he ba \\
\hline 6 & Guizhou Province & Zunyi City & Tongzi County & 28.247 & 106.844 & Anthracite & Small & Chu mi pu \\
\hline 7 & Guizhou Province & Zunyi City & Tongzi County & 28.172 & 106.838 & Bituminous & Small & Yang cun gou \\
\hline 8 & Guizhou Province & Zunyi City & Zhengan County & 28.662 & 107.420 & Bituminous & Small & An chang \\
\hline 9 & Guizhou Province & Zunyi City & $\begin{array}{l}\text { Wuchuan Autonomous } \\
\text { County }\end{array}$ & 28.625 & 107.931 & Bituminous & Small & Guan xue ba \\
\hline 10 & Guizhou Province & Zunyi City & Suiyang County & 28.271 & 107.007 & Anthracite & Small & Kuan kuo ba \\
\hline 11 & Guizhou Province & Tongren City & $\begin{array}{l}\text { Yanhe Autonomous } \\
\text { County }\end{array}$ & 28.306 & 108.425 & Bituminous & Small & Qiao jia pu \\
\hline 12 & Guizhou Province & Bijie City & Hezhang County & 27.225 & 104.659 & Anthracite & Small & Liu qu \\
\hline 13 & Guizhou Province & Bijie City & NA & 27.261 & 105.188 & Anthracite & Small & Shi gang ling \\
\hline 14 & Guizhou Province & Bijie City & NA & 27.250 & 105.141 & Anthracite & Small & Ya guan \\
\hline 15 & Guizhou Province & Bijie City & NA & 27.127 & 105.121 & Anthracite & Medium & Yin di \\
\hline 17 & Guizhou Province & Bijie City & Dafang County & 27.121 & 105.445 & Anthracite & Small & Miao li \\
\hline 18 & Guizhou Province & Bijie City & Dafang County & 27.215 & 105.711 & Anthracite & Small & Zha ping \\
\hline 19 & Guizhou Province & Bijie City & Qianxi County & 27.191 & 105.977 & Anthracite & Small & Hua wo di \\
\hline 20 & Guizhou Province & Zunyi City & NA & 27.773 & 106.963 & Anthracite & Small & Tian gou \\
\hline 21 & Guizhou Province & Zunyi City & Renhuai County & 27.705 & 106.410 & Anthracite & Large & Sang shu wan \\
\hline 22 & Guizhou Province & Zunyi City & NA & 27.631 & 106.709 & Anthracite & Small & Gao qiao \\
\hline 23 & Guizhou Province & Zunyi City & NA & 27.583 & 106.814 & Anthracite & Small & Nan bei zhen \\
\hline
\end{tabular}


Table 1. List of coal mines in China. - Continued

[Abbreviations used: dec. deg. - decimal degrees; NA - not available or not applicable]

\begin{tabular}{|c|c|c|c|c|c|c|c|c|}
\hline $\begin{array}{c}\text { ID } \\
\text { Number }\end{array}$ & $\begin{array}{c}\text { Province, National } \\
\text { Municipality, or } \\
\text { Autonomous Region }\end{array}$ & $\begin{array}{c}\text { Prefecture, Municipality, } \\
\text { Prefecture-Level City, or } \\
\text { League }\end{array}$ & $\begin{array}{l}\text { County, District or } \\
\text { County-Level City }\end{array}$ & $\begin{array}{l}\text { Latitude } \\
\text { (dec. deg.) }\end{array}$ & $\begin{array}{l}\text { Longitude } \\
\text { (dec. deg.) }\end{array}$ & Rank & $\begin{array}{l}\text { Relative } \\
\text { Mine Size }\end{array}$ & Mine Name \\
\hline 24 & Guizhou Province & Bijie City & Jinshan County & 27.478 & 106.518 & Anthracite & Medium & Lao mu kong \\
\hline 25 & Guizhou Province & Guiyang City & Xifeng County & 27.151 & 106.732 & Anthracite & Small & Nan shan \\
\hline 26 & Guizhou Province & Guiyang City & Xifeng County & 27.066 & 106.761 & Bituminous & Small & Shi guan kou \\
\hline 27 & Guizhou Province & $\begin{array}{l}\text { Qiannan Autonomous } \\
\text { Prefecture }\end{array}$ & Wengan County & 27.069 & 107.525 & Bituminous & Small & Xi ma tang \\
\hline 28 & Guizhou Province & Tongren City & Sinan County & 27.819 & 108.095 & Bituminous & Small & Feng xiang yuan \\
\hline 29 & Guizhou Province & $\begin{array}{l}\text { Qiandongnan Autonomous } \\
\text { Prefecture }\end{array}$ & Shibing County & 27.064 & 108.121 & Lignite & Small & Weng shao \\
\hline 30 & Guizhou Province & Bijie City & Hezhang County & 26.960 & 104.546 & Bituminous & Small & Ma gu \\
\hline 31 & Guizhou Province & Bijie City & $\begin{array}{l}\text { Weining Autonomous } \\
\text { County }\end{array}$ & 26.816 & 104.510 & Bituminous & Small & Jie li \\
\hline 32 & Guizhou Province & Liupanshui City & NA & 26.784 & 104.648 & Bituminous & Medium & Er tang \\
\hline 33 & Guizhou Province & Liupanshui City & NA & 26.758 & 104.896 & Bituminous & Medium & Shen xian po \\
\hline 34 & Guizhou Province & Liupanshui City & NA & 26.711 & 104.802 & Bituminous & Medium & Da he bian \\
\hline 35 & Guizhou Province & Liupanshui City & NA & 26.679 & 104.936 & Bituminous & Medium & Tu die \\
\hline 36 & Guizhou Province & Liupanshui City & NA & 26.484 & 104.809 & Bituminous & Large & Lan ba \\
\hline 37 & Guizhou Province & Liupanshui City & NA & 26.487 & 104.984 & Bituminous & Medium & Niu chang \\
\hline 38 & Guizhou Province & Liupanshui City & NA & 26.335 & 104.764 & Bituminous & Medium & Fa er \\
\hline 39 & Guizhou Province & Liupanshui City & Panxian County & 26.060 & 104.574 & Bituminous & Large & Tu cheng \\
\hline 40 & Guizhou Province & Bijie City & Nayong County & 26.719 & 105.266 & Anthracite & Small & Zhong ling \\
\hline 41 & Guizhou Province & Liupanshui City & NA & 26.597 & 105.025 & Bituminous & Small & Xiao he bian \\
\hline 42 & Guizhou Province & Liupanshui City & NA & 26.569 & 105.157 & Bituminous & Small & Bi de \\
\hline 43 & Guizhou Province & Bijie City & Zhijin County & 26.635 & 105.644 & Anthracite & Medium & A gong \\
\hline 44 & Guizhou Province & Bijie City & Zhijin County & 26.561 & 105.650 & Anthracite & Medium & Shao pu \\
\hline 45 & Guizhou Province & Anshun City & NA & 26.386 & 105.913 & Anthracite & Small & Qiao zi shan \\
\hline 46 & Guizhou Province & Bijie City & Liuzhi County & 26.232 & 105.450 & Bituminous & Small & Liu zhi \\
\hline 47 & Guizhou Province & Liupanshui City & Liuzhi County & 26.201 & 105.602 & Bituminous & Small & Da mei shan \\
\hline 48 & Guizhou Province & $\begin{array}{l}\text { Qianxinan Autonomous } \\
\text { Prefecture }\end{array}$ & Qinglong County & 26.063 & 105.097 & Bituminous & Small & Zhong ying \\
\hline 49 & Guizhou Province & Liupanshui City & Liuzhi County & 26.079 & 105.327 & Bituminous & Small & Lang dai \\
\hline 50 & Guizhou Province & Guiyang City & Xiuwen County & 26.893 & 106.738 & Bituminous & Small & Shi yan he \\
\hline 51 & Guizhou Province & Guiyang City & Xiuwen County & 26.851 & 106.705 & Bituminous & Small & Zha zho \\
\hline 52 & Guizhou Province & Guiyang City & Xiuwen County & 26.823 & 106.577 & Bituminous & Small & Xiao he gou \\
\hline 53 & Guizhou Province & Guiyang City & NA & 26.756 & 106.544 & Bituminous & Small & E jing chong \\
\hline 54 & Guizhou Province & Guiyang City & NA & 26.687 & 106.570 & Bituminous & Small & Shi dong \\
\hline
\end{tabular}


Table 1. List of coal mines in China. - Continued

[Abbreviations used: dec. deg. - decimal degrees; NA - not available or not applicable]

\begin{tabular}{|c|c|c|c|c|c|c|c|c|}
\hline $\begin{array}{c}\text { ID } \\
\text { Number }\end{array}$ & $\begin{array}{c}\text { Province, National } \\
\text { Municipality, or } \\
\text { Autonomous Region }\end{array}$ & $\begin{array}{c}\text { Prefecture, Municipality, } \\
\text { Prefecture-Level City, or } \\
\text { League }\end{array}$ & $\begin{array}{l}\text { County, District or } \\
\text { County-Level City }\end{array}$ & $\begin{array}{l}\text { Latitude } \\
\text { (dec. deg.) }\end{array}$ & $\begin{array}{l}\text { Longitude } \\
\text { (dec. deg.) }\end{array}$ & Rank & $\begin{array}{l}\text { Relative } \\
\text { Mine Size }\end{array}$ & Mine Name \\
\hline 55 & Guizhou Province & Guiyang City & Qingzhen City & 26.656 & 106.179 & Anthracite & Small & Liu chang \\
\hline 56 & Guizhou Province & Guiyang City & Qingzhen City & 26.592 & 106.326 & Anthracite & Medium & Xi guan \\
\hline 57 & Guizhou Province & Guiyang City & NA & 26.574 & 106.614 & Bituminous & Medium & Lin dong \\
\hline 58 & Guizhou Province & Anshun City & Pingba City & 26.488 & 106.191 & Anthracite & Small & Gu bao \\
\hline 59 & Guizhou Province & Guiyang City & Kaiyang City & 26.909 & 107.127 & Bituminous & Small & Yang chang \\
\hline 60 & Guizhou Province & Guiyang City & Fuquan City & 26.882 & 107.543 & Bituminous & Small & Gao shi \\
\hline 61 & Guizhou Province & $\begin{array}{l}\text { Qiannan Autonomous } \\
\text { Prefecture }\end{array}$ & Fuquan City & 26.813 & 107.442 & Bituminous & Small & Yang jia lin \\
\hline 62 & Guizhou Province & $\begin{array}{l}\text { Qiandongnan Autonomous } \\
\text { Prefecture }\end{array}$ & Huangping County & 26.766 & 107.949 & Bituminous & Small & Chong an jiang \\
\hline 63 & Guizhou Province & $\begin{array}{l}\text { Qiannan Autonomous } \\
\text { Prefecture }\end{array}$ & Longli County & 26.740 & 107.104 & Bituminous & Small & Xima jiang \\
\hline 64 & Guizhou Province & $\begin{array}{l}\text { Qiannan Autonomous } \\
\text { Prefecture }\end{array}$ & Guiding County & 26.759 & 107.191 & Bituminous & Small & Lan ni wan \\
\hline 65 & Guizhou Province & $\begin{array}{l}\text { Qiannan Autonomous } \\
\text { Prefecture }\end{array}$ & Fuquan City & 26.647 & 107.589 & Bituminous & Small & Gan ba shao \\
\hline 66 & Guizhou Province & $\begin{array}{l}\text { Qiandongnan Autonomous } \\
\text { Prefecture }\end{array}$ & Kaili City & 26.658 & 107.743 & Bituminous & Small & Hou zhuang \\
\hline 67 & Guizhou Province & $\begin{array}{l}\text { Qiandongnan Autonomous } \\
\text { Prefecture }\end{array}$ & Kaili City & 26.689 & 107.903 & Bituminous & Small & Yu dong \\
\hline 68 & Guizhou Province & $\begin{array}{l}\text { Qiandongnan Autonomous } \\
\text { Prefecture }\end{array}$ & Kaili City & 26.664 & 107.904 & Bituminous & Small & Gou chang \\
\hline 69 & Guizhou Province & $\begin{array}{l}\text { Qiandongnan Autonomous } \\
\text { Prefecture }\end{array}$ & Kaili City & 26.635 & 107.872 & Bituminous & Small & Tao zi chong \\
\hline 70 & Guizhou Province & $\begin{array}{l}\text { Qiandongnan Autonomous } \\
\text { Prefecture }\end{array}$ & Majiang County & 26.519 & 107.557 & Bituminous & Small & Da liang tian \\
\hline 71 & Guizhou Province & $\begin{array}{l}\text { Qiannan Autonomous } \\
\text { Prefecture }\end{array}$ & Duyun City & 26.411 & 107.507 & Bituminous & Small & Yang liu jie \\
\hline 72 & Guizhou Province & $\begin{array}{l}\text { Qiannan Autonomous } \\
\text { Prefecture }\end{array}$ & Duyun City & 26.387 & 107.481 & Bituminous & Small & Sha guo chang \\
\hline 73 & Guizhou Province & $\begin{array}{l}\text { Qiannan Autonomous } \\
\text { Prefecture }\end{array}$ & Duyun City & 26.362 & 107.546 & Bituminous & Small & Tie chong \\
\hline 74 & Guizhou Province & $\begin{array}{l}\text { Qiannan Autonomous } \\
\text { Prefecture }\end{array}$ & Duyun City & 26.321 & 107.558 & Bituminous & Small & Da wo dang \\
\hline 75 & Guizhou Province & $\begin{array}{l}\text { Qiandongnan Autonomous } \\
\text { Prefecture }\end{array}$ & Tianzhu County & 26.989 & 109.233 & Bituminous & Small & Bang dong \\
\hline 76 & Guizhou Province & Liupanshui City & Panxian County & 25.932 & 104.636 & Bituminous & Medium & Zhao zi jiang \\
\hline 77 & Guizhou Province & Liupanshui City & Panxian County & 25.944 & 104.822 & Bituminous & Large & Jiu cun \\
\hline
\end{tabular}


Table 1. List of coal mines in China.-Continued

[Abbreviations used: dec. deg. - decimal degrees; NA - not available or not applicable]

\begin{tabular}{|c|c|c|c|c|c|c|c|c|}
\hline $\begin{array}{l}\text { ID } \\
\text { Number }\end{array}$ & $\begin{array}{l}\text { Province, National } \\
\text { Municipality, or } \\
\text { Autonomous Region }\end{array}$ & $\begin{array}{l}\text { Prefecture, Municipality, } \\
\text { Prefecture-Level City, or } \\
\text { League }\end{array}$ & $\begin{array}{l}\text { County, District or } \\
\text { County-Level City }\end{array}$ & $\begin{array}{l}\text { Latitude } \\
\text { (dec. deg.) }\end{array}$ & $\begin{array}{l}\text { Longitude } \\
\text { (dec. deg.) }\end{array}$ & Rank & $\begin{array}{l}\text { Relative } \\
\text { Mine Size }\end{array}$ & Mine Name \\
\hline 78 & Guizhou Province & $\begin{array}{l}\text { Qianxinan Autonomous } \\
\text { Prefecture }\end{array}$ & Puan County & 25.869 & 104.947 & Bituminous & Small & Xin tian \\
\hline 79 & Guizhou Province & Liupanshui City & Panxian County & 25.784 & 104.478 & Bituminous & Large & Zhi chang \\
\hline 80 & Guizhou Province & $\begin{array}{l}\text { Qianxinan Autonomous } \\
\text { Prefecture }\end{array}$ & Puan County & 25.762 & 104.840 & Bituminous & Medium & Wang guan tun \\
\hline 81 & Guizhou Province & Liupanshui City & Panxian County & 25.690 & 104.622 & Bituminous & Medium & Shui tang \\
\hline 82 & Guizhou Province & Liupanshui City & Panxian County & 25.652 & 104.504 & Bituminous & Medium & Jia zhu jing \\
\hline 83 & Guizhou Province & Liupanshui City & Panxian County & 25.533 & 104.677 & Bituminous & Large & Bo tu \\
\hline 84 & Guizhou Province & $\begin{array}{l}\text { Qianxinan Autonomous } \\
\text { Prefecture }\end{array}$ & Qinglong County & 25.815 & 105.176 & Anthracite & Small & Xiao zhai \\
\hline 85 & Guizhou Province & $\begin{array}{l}\text { Qianxinan Autonomous } \\
\text { Prefecture }\end{array}$ & Xingren County & 25.530 & 105.516 & Anthracite & Small & Lan mu chang \\
\hline 86 & Guizhou Province & $\begin{array}{l}\text { Qianxinan Autonomous } \\
\text { Prefecture }\end{array}$ & Xingren County & 25.381 & 105.139 & Anthracite & Medium & Dae kou \\
\hline 87 & Guizhou Province & $\begin{array}{l}\text { Qianxinan Autonomous } \\
\text { Prefecture }\end{array}$ & Xingren County & 25.408 & 105.471 & Bituminous & Medium & Long tou shan \\
\hline 88 & Guizhou Province & $\begin{array}{l}\text { Qianxinan Autonomous } \\
\text { Prefecture }\end{array}$ & Anlong County & 25.298 & 105.303 & Anthracite & Medium & Ge tang \\
\hline 89 & Guizhou Province & $\begin{array}{l}\text { Qiannan Autonomous } \\
\text { Prefecture }\end{array}$ & Libo County & 25.382 & 108.079 & Bituminous & Small & Mao lan \\
\hline 90 & Guizhou Province & $\begin{array}{l}\text { Qianxinan Autonomous } \\
\text { Prefecture }\end{array}$ & Xinyi County & 24.991 & 104.777 & Anthracite & Medium & Qi she \\
\hline 9 & Hainan Province & Danzhou City & NA & 19.710 & 109.437 & Lignite & Medium & Chang po \\
\hline 28 & Hainan Province & Haikou City & Qiongshan District & 19.637 & 110.457 & Lignite & Small & Chang chang \\
\hline 5 & Hebei Province & Chengde City & $\begin{array}{l}\text { Weichang Autonomous } \\
\text { County }\end{array}$ & 42.070 & 117.914 & Anthracite & Small & Zhao yang wan zi \\
\hline 6 & Hebei Province & Chengde City & $\begin{array}{l}\text { Weichang Autonomous } \\
\text { County }\end{array}$ & 42.048 & 117.906 & Anthracite & Small & Shui quan gou \\
\hline 7 & Hebei Province & Zhangjiakou City & Kangbao County & 41.495 & 114.610 & Bituminous & Small & Tu cheng zi \\
\hline 8 & Hebei Province & Zhangjiakou City & Zhangbei County & 41.140 & 114.752 & Lignite & Small & $\begin{array}{l}\text { Cheng guan } \\
\text { Exploration Area }\end{array}$ \\
\hline 9 & Hebei Province & Zhangjiakou City & Shangyi County & 41.019 & 114.179 & Bituminous & Small & $\begin{array}{l}\text { Hong tu liang } \\
\text { Exploration Area, } \\
\text { Da ba Well Field }\end{array}$ \\
\hline 10 & Hebei Province & Zhangjiakou City & Chongli County & 41.004 & 114.835 & Lignite & Medium & Wu shi jia zi \\
\hline 11 & Hebei Province & Zhangjiakou City & Guyuan County & 41.873 & 115.797 & Lignite & Medium & Yu shu gou \\
\hline 12 & Hebei Province & Chengde City & Fengning County & 41.847 & 116.677 & Lignite & Small & Qing shi \\
\hline
\end{tabular}


Table 1. List of coal mines in China.-Continued

[Abbreviations used: dec. deg. - decimal degrees; NA - not available or not applicable]

\begin{tabular}{|c|c|c|c|c|c|c|c|c|}
\hline $\begin{array}{c}\text { ID } \\
\text { Number }\end{array}$ & $\begin{array}{c}\text { Province, National } \\
\text { Municipality, or } \\
\text { Autonomous Region }\end{array}$ & $\begin{array}{c}\text { Prefecture, Municipality, } \\
\text { Prefecture-Level City, or } \\
\text { League }\end{array}$ & $\begin{array}{l}\text { County, District or } \\
\text { County-Level City }\end{array}$ & $\begin{array}{l}\text { Latitude } \\
\text { (dec. deg.) }\end{array}$ & $\begin{array}{l}\text { Longitude } \\
\text { (dec. deg.) }\end{array}$ & Rank & $\begin{array}{l}\text { Relative } \\
\text { Mine Size }\end{array}$ & Mine Name \\
\hline 16 & Hebei Province & Chengde City & Fengning County & 41.270 & 117.298 & Lignite & Small & Xia ba \\
\hline 17 & Hebei Province & Chengde City & Longhua County & 41.336 & 117.710 & Bituminous & Small & Tai shan \\
\hline 19 & Hebei Province & & $\begin{array}{l}\text { Weichang Autonomous } \\
\text { County }\end{array}$ & 41.966 & 118.133 & Bituminous & Small & Xiao wei zi gou \\
\hline 20 & Hebei Province & Chengde City & Shangyi County & 40.999 & 114.162 & Anthracite & Small & $\begin{array}{l}\text { Hong tu liang } \\
\text { Exploration Area, } \\
\text { Da yang po } \\
\text { Well Field }\end{array}$ \\
\hline 21 & Hebei Province & Zhangjiakou City & Wanquan County & 40.895 & 114.693 & Lignite & Medium & $\begin{array}{l}\text { Huang jia bao } \\
\text { Exploration Area }\end{array}$ \\
\hline 22 & Hebei Province & Zhangjiakou City & Wanquan County & 40.870 & 114.712 & Lignite & Medium & $\begin{array}{l}\text { Cheng xi } \\
\text { Exploration Area }\end{array}$ \\
\hline 23 & Hebei Province & Zhangjiakou City & Wanquan County & 40.839 & 114.667 & Lignite & Small & \\
\hline 24 & Hebei Province & Zhangjiakou City & Wanquan County & 40.799 & 114.788 & Lignite & Medium & $\begin{array}{l}\text { Xuan ping bao } \\
\text { Exploration Area }\end{array}$ \\
\hline 25 & Hebei Province & Zhangjiakou City & Xuanhua County & 40.597 & 115.128 & Bituminous & Small & $\begin{array}{l}\text { Xia hua yuan Coal } \\
\text { Mine, Xuan dong } \\
\text { No. } 1 \text { Well }\end{array}$ \\
\hline 26 & Hebei Province & Zhangjiakou City & Xuanhua County & 40.613 & 115.307 & Bituminous & Small & Hei dai shan \\
\hline 27 & Hebei Province & Zhangjiakou City & NA & 40.490 & 115.286 & Bituminous & Small & $\begin{array}{l}\text { Xia hua yuan } \\
\text { No. } 4 \text { Well }\end{array}$ \\
\hline 28 & Hebei Province & Zhangjiakou City & NA & 40.479 & 115.296 & Bituminous & Small & $\begin{array}{l}\text { Xia hua yuan, } \\
\text { Ji ming shan }\end{array}$ \\
\hline 29 & Hebei Province & Zhangjiakou City & NA & 40.529 & 115.394 & Anthracite & Small & $\begin{array}{l}\text { Ba bao shan Coal } \\
\text { Mine, No. } 71 \\
\text { Well Field }\end{array}$ \\
\hline 30 & Hebei Province & Zhangjiakou City & NA & 40.482 & 115.476 & Bituminous & Small & $\begin{array}{l}\text { Ba bao shan Coal } \\
\text { Mine, Miao liang } \\
\text { shan Well Field }\end{array}$ \\
\hline 31 & Hebei Province & Zhangjiakou City & NA & 40.471 & 115.235 & Bituminous & Small & $\begin{array}{l}\text { Xia hua yuan, } \\
\text { Qian shan }\end{array}$ \\
\hline 32 & Hebei Province & Zhangjiakou City & Zhuolu County & 40.458 & 115.217 & Bituminous & Small & Hu zhuang \\
\hline 33 & Hebei Province & Zhangjiakou City & NA & 40.458 & 115.217 & Bituminous & Small & $\begin{array}{c}\text { Xia hua yuan, Yu dai } \\
\text { shan, Xiang xie xi } \\
\text { Exploration Area }\end{array}$ \\
\hline 34 & Hebei Province & Zhangjiakou City & NA & 40.454 & 115.260 & Bituminous & Small & $\begin{array}{l}\text { Xia hua yuan Coal } \\
\text { Field, Yu dai shan } \\
\text { Well Field }\end{array}$ \\
\hline
\end{tabular}


Table 1. List of coal mines in China.-Continued

[Abbreviations used: dec. deg. - decimal degrees; NA - not available or not applicable]

\begin{tabular}{|c|c|c|c|c|c|c|c|c|}
\hline $\begin{array}{l}\text { ID } \\
\text { Number }\end{array}$ & $\begin{array}{l}\text { Province, National } \\
\text { Municipality, or } \\
\text { Autonomous Region }\end{array}$ & $\begin{array}{l}\text { Prefecture, Municipality, } \\
\text { Prefecture-Level City, or } \\
\text { League }\end{array}$ & $\begin{array}{l}\text { County, District or } \\
\text { County-Level City }\end{array}$ & $\begin{array}{l}\text { Latitude } \\
\text { (dec. deg.) }\end{array}$ & $\begin{array}{l}\text { Longitude } \\
\text { (dec. deg.) }\end{array}$ & Rank & $\begin{array}{l}\text { Relative } \\
\text { Mine Size }\end{array}$ & Mine Name \\
\hline 35 & Hebei Province & Zhangjiakou City & NA & 40.454 & 115.260 & Bituminous & Small & $\begin{array}{l}\text { Xia hua yuan Coal } \\
\text { Field, Xing long } \\
\text { shan Well Field }\end{array}$ \\
\hline 36 & Hebei Province & Zhangjiakou City & NA & 40.454 & 115.260 & Bituminous & Small & $\begin{array}{l}\text { Xia hua yuan Coal } \\
\text { Field, Yu dai shan } \\
\text { No. } 3 \text { Well Field }\end{array}$ \\
\hline 37 & Hebei Province & Zhangjiakou City & Zhuolu County & 40.350 & 115.035 & Bituminous & Small & Xi shan po Well Field \\
\hline 38 & Hebei Province & Zhangjiakou City & Zhuolu County & 40.350 & 115.035 & Bituminous & Small & $\begin{array}{l}\text { Huang tu wan } \\
\text { Well Field }\end{array}$ \\
\hline 39 & Hebei Province & Zhangjiakou City & Zhuolu County & 40.350 & 115.035 & Bituminous & Small & Xi si wan Well Field \\
\hline 40 & Hebei Province & Langfang City & Sanhe County & 40.006 & 116.946 & Anthracite & Large & $\begin{array}{l}\text { Shen zhuang } \\
\text { Well Field }\end{array}$ \\
\hline 41 & Hebei Province & Langfang City & Sanhe County & 40.014 & 116.843 & Anthracite & Medium & $\begin{array}{l}\text { Kang jia wan, Da fu } \\
\text { zhuang Well Field }\end{array}$ \\
\hline 42 & Hebei Province & Chengde City & Luanping County & 40.690 & 117.462 & Bituminous & Small & Lao wa \\
\hline 43 & Hebei Province & Chengde City & Xinglong County & 40.496 & 117.531 & Bituminous & Small & Ke li mu \\
\hline 44 & Hebei Province & Chengde City & Xinglong County & 40.511 & 117.578 & Bituminous & Small & Ping an bao \\
\hline 45 & Hebei Province & Chengde City & $\mathrm{NA}$ & 40.523 & 117.591 & Bituminous & Small & $\begin{array}{l}\text { Bei ma quan zi } \\
\text { Well Field }\end{array}$ \\
\hline 46 & Hebei Province & Chengde City & NA & 40.546 & 117.611 & Bituminous & Small & $\begin{array}{l}\text { Nan ma quan zi } \\
\text { Well Field }\end{array}$ \\
\hline 47 & Hebei Province & Chengde City & NA & 40.546 & 117.611 & Bituminous & Small & $\begin{array}{l}\text { Mei ling gou Well } \\
\text { Field, Ying zi No. } 1 \\
\text { Potential Area }\end{array}$ \\
\hline 48 & Hebei Province & Chengde City & NA & 40.545 & 117.673 & Bituminous & Small & $\begin{array}{l}\text { Lao ye miao No. } 2 \\
\text { Well Field }\end{array}$ \\
\hline 49 & Hebei Province & Chengde City & NA & 40.470 & 117.616 & Bituminous & Small & $\begin{array}{l}\text { Lao ye miao No. } 1 \\
\text { Well Field }\end{array}$ \\
\hline 50 & Hebei Province & Chengde City & NA & 40.598 & 117.741 & Bituminous & Small & $\begin{array}{l}\text { Huo shen miao } \\
\text { Well Field }\end{array}$ \\
\hline 51 & Hebei Province & Chengde City & Xinglong County & 40.543 & 117.732 & Bituminous & Small & Zao zi ling \\
\hline 52 & Hebei Province & Chengde City & Xinglong County & 40.524 & 117.769 & Bituminous & Small & Men zi gou \\
\hline 53 & Hebei Province & Chengde City & $\mathrm{NA}$ & 40.472 & 117.686 & Anthracite & Small & $\begin{array}{l}\text { Dong feng Coal Mine, } \\
\text { North Mountain } \\
\text { Pit Mouth }\end{array}$ \\
\hline 54 & Hebei Province & Chengde City & NA & 40.470 & 117.757 & Bituminous & Small & $\begin{array}{l}\text { Dong feng Coal Mine, } \\
\text { South Mountain } \\
\text { Pit Mouth }\end{array}$ \\
\hline
\end{tabular}


Table 1. List of coal mines in China. - Continued

[Abbreviations used: dec. deg. - decimal degrees; NA - not available or not applicable]

\begin{tabular}{|c|c|c|c|c|c|c|c|c|}
\hline $\begin{array}{c}\text { ID } \\
\text { Number }\end{array}$ & $\begin{array}{l}\text { Province, National } \\
\text { Municipality, or } \\
\text { Autonomous Region }\end{array}$ & $\begin{array}{l}\text { Prefecture, Municipality, } \\
\text { Prefecture-Level City, or } \\
\text { League }\end{array}$ & $\begin{array}{l}\text { County, District or } \\
\text { County-Level City }\end{array}$ & $\begin{array}{l}\text { Latitude } \\
\text { (dec. deg.) }\end{array}$ & $\begin{array}{l}\text { Longitude } \\
\text { (dec. deg.) }\end{array}$ & Rank & $\begin{array}{l}\text { Relative } \\
\text { Mine Size }\end{array}$ & Mine Name \\
\hline 55 & Hebei Province & Chengde City & Pingquan County & 40.969 & 118.834 & Bituminous & Small & Shi zi miao \\
\hline 56 & Hebei Province & Chengde City & Pingquan County & 40.977 & 118.877 & Bituminous & Small & Da lie shan \\
\hline 57 & Hebei Province & Chengde City & Pingquan County & 40.978 & 118.875 & Bituminous & Small & Yang shu ling \\
\hline 58 & Hebei Province & Chengde City & Pingquan County & 40.884 & 118.862 & Bituminous & Small & $\begin{array}{l}\text { Ying shou ying zi } \\
\text { Exploration Area }\end{array}$ \\
\hline 59 & Hebei Province & Chengde City & Pingquan County & 40.861 & 118.805 & Bituminous & Small & Song shu tai \\
\hline 60 & Hebei Province & Chengde City & Pingquan County & 40.838 & 118.567 & Bituminous & Small & Wei chang gou \\
\hline 61 & Hebei Province & Chengde City & NA & 40.822 & 118.487 & Anthracite & Small & Da guo zhang zi \\
\hline 62 & Hebei Province & Chengde City & NA & 40.808 & 118.232 & Anthracite & Small & Wu chang \\
\hline 63 & Hebei Province & Chengde City & NA & 40.629 & 118.233 & Bituminous & Small & Nuan er he \\
\hline 64 & Hebei Province & Chengde City & $\begin{array}{l}\text { Kuancheng } \\
\text { Autonomous County }\end{array}$ & 40.638 & 118.248 & Bituminous & Small & $\begin{array}{l}\text { Miao liang } \\
\text { Exploration Area }\end{array}$ \\
\hline 65 & Hebei Province & Chengde City & $\begin{array}{l}\text { Kuancheng } \\
\text { Autonomous County }\end{array}$ & 40.646 & 118.489 & Bituminous & Small & Gan yao gou \\
\hline 67 & Hebei Province & Qinhuangdao City & Qinglong County & 40.197 & 119.547 & Anthracite & Small & Chang cheng \\
\hline 68 & Hebei Province & Qinhuangdao City & Qinglong County & 40.176 & 119.527 & Anthracite & Small & $\mathrm{Na} z i \mathrm{yu}$ \\
\hline 69 & Hebei Province & Qinhuangdao City & NA & 40.164 & 119.547 & Anthracite & Small & $\begin{array}{l}\text { Liu jiang Coal Mine, } \\
\text { Yi yuan kou } \\
\text { Well Field }\end{array}$ \\
\hline 70 & Hebei Province & Qinhuangdao City & Funing County & 40.142 & 119.526 & Anthracite & Small & $\begin{array}{l}\text { Wang zhuang } \\
\text { Well Field }\end{array}$ \\
\hline 71 & Hebei Province & Qinhuangdao City & NA & 40.144 & 119.583 & Anthracite & Small & $\begin{array}{l}\text { Liu jiang Coal Mine, } \\
\text { Ma ti ling } \\
\text { Well Field }\end{array}$ \\
\hline 72 & Hebei Province & Qinhuangdao City & NA & 40.130 & 119.583 & Anthracite & Small & $\begin{array}{l}\text { Liu jiang Coal Mine, } \\
\text { Chang cheng } \\
\text { Well Field }\end{array}$ \\
\hline 73 & Hebei Province & Qinhuangdao City & NA & 40.099 & 119.568 & Anthracite & Small & Cao shan \\
\hline 74 & Hebei Province & Qinhuangdao City & NA & 40.079 & 119.554 & Anthracite & Small & $\begin{array}{l}\text { Liu jiang Coal Mine, } \\
\text { Lao liu jiang } \\
\text { Well Field }\end{array}$ \\
\hline 75 & Hebei Province & Qinhuangdao City & NA & 40.079 & 119.554 & Anthracite & Small & $\begin{array}{l}\text { Liu jiang Coal Mine, } \\
\text { Da cao gou } \\
\text { Well Field }\end{array}$ \\
\hline 76 & Hebei Province & Handan City & Wei County & 39.978 & 114.681 & Bituminous & Large & $\begin{array}{l}\text { De sheng zhuang } \\
\text { Exploration Area }\end{array}$ \\
\hline
\end{tabular}


Table 1. List of coal mines in China. - Continued

[Abbreviations used: dec. deg. - decimal degrees; NA - not available or not applicable]

\begin{tabular}{|c|c|c|c|c|c|c|c|c|}
\hline $\begin{array}{c}\text { ID } \\
\text { Number }\end{array}$ & $\begin{array}{c}\text { Province, National } \\
\text { Municipality, or } \\
\text { Autonomous Region }\end{array}$ & $\begin{array}{c}\text { Prefecture, Municipality, } \\
\text { Prefecture-Level City, or } \\
\text { League }\end{array}$ & $\begin{array}{l}\text { County, District or } \\
\text { County-Level City }\end{array}$ & $\begin{array}{l}\text { Latitude } \\
\text { (dec. deg.) }\end{array}$ & $\begin{array}{l}\text { Longitude } \\
\text { (dec. deg.) }\end{array}$ & Rank & $\begin{array}{l}\text { Relative } \\
\text { Mine Size }\end{array}$ & Mine Name \\
\hline 77 & Hebei Province & Handan City & Wei County & 39.958 & 114.473 & Bituminous & Medium & $\begin{array}{l}\text { Cui jia zhai Coal } \\
\text { Mine, Eastern Well } \\
\text { Field of Shui yu }\end{array}$ \\
\hline 78 & Hebei Province & Zhangjiakou City & Yangyuan County & 39.947 & 114.303 & Bituminous & Small & Yang yuan \\
\hline 79 & Hebei Province & Handan City & Wei County & 39.932 & 114.282 & Bituminous & Small & $\begin{array}{l}\text { Zheng gou wan } \\
\text { Well Field }\end{array}$ \\
\hline 80 & Hebei Province & Handan City & Wei County & 39.930 & 114.415 & Bituminous & Medium & $\begin{array}{l}\text { Cui jia zhai Coal } \\
\text { Mine, Western Well } \\
\text { Field of Shui yu }\end{array}$ \\
\hline 81 & Hebei Province & Handan City & Wei County & 39.928 & 114.452 & Bituminous & Small & $\begin{array}{l}\text { Cui jia zhai Coal } \\
\text { Mine, Western Well } \\
\text { Field of Cui jia zhai }\end{array}$ \\
\hline 82 & Hebei Province & Handan City & Wei County & 39.909 & 114.494 & Bituminous & Large & Cui jia zhai Well Field \\
\hline 83 & Hebei Province & Handan City & Wei County & 39.909 & 114.494 & Bituminous & Large & $\begin{array}{l}\text { Shan hou cun } \\
\text { Well Field }\end{array}$ \\
\hline 84 & Hebei Province & Handan City & Wei County & 39.918 & 114.590 & Bituminous & Large & $\begin{array}{l}\text { Bei yang zhuang } \\
\text { Well Field }\end{array}$ \\
\hline 85 & Hebei Province & Handan City & Wei County & 39.899 & 114.407 & Bituminous & Small & $\begin{array}{l}\text { Xi xi zhuang } \\
\text { Well Field }\end{array}$ \\
\hline 86 & Hebei Province & Handan City & Wei County & 39.883 & 114.451 & Bituminous & Medium & $\begin{array}{l}\text { Nan liu zhuang } \\
\text { Well Field }\end{array}$ \\
\hline 87 & Hebei Province & Handan City & Wei County & 39.869 & 114.402 & Bituminous & Small & $\begin{array}{l}\text { Meng jia bao Coal } \\
\text { Field, Guai xi liang } \\
\text { Well Field }\end{array}$ \\
\hline 88 & Hebei Province & Handan City & Wei County & 39.858 & 114.421 & Bituminous & Small & $\begin{array}{l}\text { Yu feng shan } \\
\text { Well Field }\end{array}$ \\
\hline 89 & Hebei Province & Baoding City & Laiyuan County & 39.322 & 114.641 & Lignite & Small & Dou jun wan \\
\hline 90 & Hebei Province & Baoding City & Fuping County & 39.127 & 114.408 & Anthracite & Small & Tan hui pu \\
\hline 91 & Hebei Province & Baoding City & Laishui County & 39.450 & 115.632 & Anthracite & Small & Lei zi Well Field \\
\hline 92 & Hebei Province & Baoding City & Yixian County & 39.429 & 115.562 & Anthracite & Small & Fen zhuang \\
\hline 93 & Hebei Province & Baoding City & Laishui County & 39.429 & 115.562 & Anthracite & Small & Yong yang No. 1 Well \\
\hline 94 & Hebei Province & Tangshan City & Yutian County & 39.845 & 117.563 & Bituminous & Small & $\begin{array}{l}\text { Li zhuang zi } \\
\text { Exploration Area }\end{array}$ \\
\hline 95 & Hebei Province & Tangshan City & Yutian County & 39.847 & 117.634 & Bituminous & Large & Kai luan, Lin nan cang \\
\hline 97 & Hebei Province & Tangshan City & NA & 39.632 & 117.951 & Bituminous & Medium & $\begin{array}{l}\text { Xin jun tun } \\
\text { Exploration Area }\end{array}$ \\
\hline 98 & Hebei Province & Tangshan City & NA & 39.618 & 117.998 & Bituminous & Large & Dong huan tuo \\
\hline 99 & Hebei Province & Tangshan City & NA & 39.779 & 118.200 & Bituminous & Medium & Jing ge zhuang \\
\hline
\end{tabular}


Table 1. List of coal mines in China. - Continued

[Abbreviations used: dec. deg. - decimal degrees; NA - not available or not applicable]

\begin{tabular}{|c|c|c|c|c|c|c|c|c|}
\hline $\begin{array}{c}\text { ID } \\
\text { Number }\end{array}$ & $\begin{array}{c}\text { Province, National } \\
\text { Municipality, or } \\
\text { Autonomous Region }\end{array}$ & $\begin{array}{l}\text { Prefecture, Municipality, } \\
\text { Prefecture-Level City, or } \\
\text { League }\end{array}$ & $\begin{array}{l}\text { County, District or } \\
\text { County-Level City }\end{array}$ & $\begin{array}{l}\text { Latitude } \\
\text { (dec. deg.) }\end{array}$ & $\begin{array}{l}\text { Longitude } \\
\text { (dec. deg.) }\end{array}$ & Rank & $\begin{array}{l}\text { Relative } \\
\text { Mine Size }\end{array}$ & Mine Name \\
\hline 100 & Hebei Province & Tangshan City & Luanxian County & 39.804 & 118.345 & Bituminous & Small & \\
\hline 101 & Hebei Province & Tangshan City & NA & 39.791 & 118.369 & Bituminous & Large & Zhao ge zhuang \\
\hline 102 & Hebei Province & Tangshan City & NA & 39.777 & 118.306 & Bituminous & Small & $\begin{array}{l}\text { East Mining Area } \\
\text { Qian xi }\end{array}$ \\
\hline 103 & Hebei Province & Tangshan City & NA & 39.772 & 118.288 & Bituminous & Small & $\begin{array}{l}\text { Zhao ge zhuang Mine, } \\
\text { Wei shan Well Field }\end{array}$ \\
\hline 104 & Hebei Province & Tangshan City & NA & 39.775 & 118.425 & Bituminous & Medium & Tang jia zhuang \\
\hline 105 & Hebei Province & Tangshan City & NA & 39.754 & 118.227 & Bituminous & Small & $\begin{array}{l}\text { Liu guan tun } \\
\text { Exploration Area }\end{array}$ \\
\hline 106 & Hebei Province & Baoding City & $\begin{array}{l}\text { Laishui County, } \\
\text { Zhaoge Village }\end{array}$ & 39.758 & 118.347 & Bituminous & Small & $\begin{array}{l}\text { Kai luan Group, } \\
\text { Zhaogezhuang } \\
\text { Mine, Gold Mining } \\
\text { Area, Eastern } \\
\text { Mining Area, No. } 1 \\
\text { Coal Mine }\end{array}$ \\
\hline 107 & Hebei Province & Tangshan City & Zunhua County & 39.767 & 118.457 & Bituminous & Small & \\
\hline 108 & Hebei Province & Tangshan City & NA & 39.773 & 118.470 & Bituminous & Small & $\begin{array}{l}\text { Eastern Mining Area, } \\
\text { Feng run, } \\
\text { Hong xing }\end{array}$ \\
\hline 109 & Hebei Province & Tangshan City & NA & 39.769 & 118.484 & Bituminous & Small & $\begin{array}{l}\text { Tang jia zhuang Mine, } \\
\text { Zun hua No. } 2 \text { Mine }\end{array}$ \\
\hline 110 & Hebei Province & Tangshan City & Laoting County & 39.768 & 118.499 & Bituminous & Small & Yue jin \\
\hline 111 & Hebei Province & Tangshan City & NA & 39.739 & 118.261 & Bituminous & Small & Yin zi gou \\
\hline 112 & Hebei Province & Tangshan City & NA & 39.748 & 118.396 & Bituminous & Small & Luan nan xin Well \\
\hline 113 & Hebei Province & Tangshan City & NA & 39.748 & 118.411 & Bituminous & Large & Lin $x i$ \\
\hline 114 & Hebei Province & Tangshan City & NA & 39.730 & 118.212 & Bituminous & Medium & $\begin{array}{l}\text { Ma jia gou Mine } \\
\text { Western wing }\end{array}$ \\
\hline 115 & Hebei Province & Qinhuangdao City & Lulong County & 39.725 & 118.226 & Bituminous & Small & Lu long \\
\hline 116 & Hebei Province & Tangshan City & NA & 39.723 & 118.241 & Bituminous & Small & Qu zhuang \\
\hline 117 & Hebei Province & Tangshan City & NA & 39.717 & 118.233 & Bituminous & Small & $\begin{array}{l}\text { Ma jia gou Mine, } \\
\text { Eastern wing }\end{array}$ \\
\hline 118 & Hebei Province & Tangshan City & NA & 39.709 & 118.229 & Bituminous & Medium & $\begin{array}{l}\text { Ma jia gou Mine, } \\
\text { Yan shen } \\
\text { Exploration Area }\end{array}$ \\
\hline 119 & Hebei Province & Tangshan City & NA & 39.732 & 118.377 & Bituminous & Small & $\begin{array}{l}\text { Eastern Mining Area, } \\
\text { Qian an No. } 51\end{array}$ \\
\hline
\end{tabular}


Table 1. List of coal mines in China. - Continued

[Abbreviations used: dec. deg. - decimal degrees; NA - not available or not applicable]

\begin{tabular}{|c|c|c|c|c|c|c|c|c|}
\hline $\begin{array}{c}\text { ID } \\
\text { Number }\end{array}$ & $\begin{array}{l}\text { Province, National } \\
\text { Municipality, or } \\
\text { Autonomous Region }\end{array}$ & $\begin{array}{c}\text { Prefecture, Municipality, } \\
\text { Prefecture-Level City, or } \\
\text { League }\end{array}$ & $\begin{array}{l}\text { County, District or } \\
\text { County-Level City }\end{array}$ & $\begin{array}{l}\text { Latitude } \\
\text { (dec. deg.) }\end{array}$ & $\begin{array}{l}\text { Longitude } \\
\text { (dec. deg.) }\end{array}$ & Rank & $\begin{array}{l}\text { Relative } \\
\text { Mine Size }\end{array}$ & Mine Name \\
\hline 120 & Hebei Province & Tangshan City & NA & 39.717 & 118.377 & Bituminous & Small & $\begin{array}{l}\text { Kai luan Lin xi Mine, } \\
\text { Western wing of } \\
\text { Eastern Mining } \\
\text { Area No. } 2 \\
\text { Coal Mine }\end{array}$ \\
\hline 121 & Hebei Province & Tangshan City & NA & 39.705 & 118.198 & Bituminous & Small & $\begin{array}{l}\text { Guo ge zhuang } \\
\text { Well Field }\end{array}$ \\
\hline 122 & Hebei Province & Tangshan City & Fengrun District & 39.689 & 118.173 & Bituminous & Small & Xi gang yao \\
\hline 123 & Hebei Province & Tangshan City & NA & 39.690 & 118.190 & Bituminous & Small & Dong gang yao \\
\hline 124 & Hebei Province & Tangshan City & NA & 39.686 & 118.218 & Bituminous & Small & Dong feng \\
\hline 125 & Hebei Province & Tangshan City & NA & 39.703 & 118.371 & Bituminous & Large & Lu jia tuo \\
\hline 126 & Hebei Province & Qinhuangdao City & Changli County & 39.684 & 118.355 & Bituminous & Small & Xin Well \\
\hline 127 & Hebei Province & Tangshan City & NA & 39.686 & 118.418 & Bituminous & Large & Fan ge zhuang \\
\hline 128 & Hebei Province & Tangshan City & NA & 39.669 & 118.196 & Bituminous & Small & Kai ping \\
\hline 129 & Hebei Province & Tangshan City & NA & 39.653 & 118.198 & Bituminous & Large & Tang shan \\
\hline 130 & Hebei Province & Tangshan City & NA & 39.647 & 118.176 & Bituminous & Small & Feng nan \\
\hline 131 & Hebei Province & Tangshan City & NA & 39.638 & 118.170 & Bituminous & Small & $\begin{array}{l}\text { Feng nan Coal Mine, } \\
\text { Nan liu zhuang } \\
\text { Well Field }\end{array}$ \\
\hline 132 & Hebei Province & Tangshan City & NA & 39.626 & 118.186 & Bituminous & Small & Liu zhuang \\
\hline 133 & Hebei Province & Tangshan City & NA & 39.641 & 118.343 & Bituminous & Large & $\begin{array}{l}\text { Qian jia ying } \\
\text { Well Field }\end{array}$ \\
\hline 134 & Hebei Province & Tangshan City & NA & 39.606 & 118.416 & Bituminous & Medium & $\begin{array}{l}\text { Bi ge zhuang, } \\
\text { Xian xie Area }\end{array}$ \\
\hline 135 & Hebei Province & Baoding City & NA & 38.867 & 114.771 & Anthracite & Small & Mi cheng \\
\hline 136 & Hebei Province & Baoding City & Quyang County & 38.811 & 114.527 & Anthracite & Small & No. 2 Coal Mine \\
\hline 137 & Hebei Province & Baoding City & Quyang County & 38.864 & 114.609 & Anthracite & Small & $\begin{array}{l}\text { Gang bei } \\
\text { Exploration Area }\end{array}$ \\
\hline 138 & Hebei Province & Baoding City & Quyang County & 38.804 & 114.720 & Anthracite & Small & Xing zi gou \\
\hline 139 & Hebei Province & Baoding City & Quyang County & 38.764 & 114.722 & Anthracite & Small & $\begin{array}{l}\text { Ling shan Coal Mine, } \\
\text { No. } 1 \text { Well }\end{array}$ \\
\hline 140 & Hebei Province & Baoding City & Quyang County & 38.764 & 114.722 & Anthracite & Small & $\begin{array}{l}\text { Ling shan Coal Mine, } \\
\text { No. } 3 \text { Well }\end{array}$ \\
\hline 141 & Hebei Province & Baoding City & Quyang County & 38.764 & 114.722 & Anthracite & Small & $\begin{array}{l}\text { Ling shan Coal Mine, } \\
\text { No. } 4 \text { Well }\end{array}$ \\
\hline 142 & Hebei Province & Baoding City & Quyang County & 38.764 & 114.722 & Anthracite & Small & $\begin{array}{l}\text { Ling shan Coal Mine, } \\
\text { No. } 5 \text { Well }\end{array}$ \\
\hline 143 & Hebei Province & Baoding City & Quyang County & 38.754 & 114.573 & Bituminous & Small & No. 4 Coal Mine \\
\hline
\end{tabular}


Table 1. List of coal mines in China.-Continued

[Abbreviations used: dec. deg. - decimal degrees; NA - not available or not applicable]

\begin{tabular}{|c|c|c|c|c|c|c|c|c|}
\hline $\begin{array}{c}\text { ID } \\
\text { Number }\end{array}$ & $\begin{array}{l}\text { Province, National } \\
\text { Municipality, or } \\
\text { Autonomous Region }\end{array}$ & $\begin{array}{c}\text { Prefecture, Municipality, } \\
\text { Prefecture-Level City, or } \\
\text { League }\end{array}$ & $\begin{array}{l}\text { County, District or } \\
\text { County-Level City }\end{array}$ & $\begin{array}{l}\text { Latitude } \\
\text { (dec. deg.) }\end{array}$ & $\begin{array}{l}\text { Longitude } \\
\text { (dec. deg.) }\end{array}$ & Rank & $\begin{array}{l}\text { Relative } \\
\text { Mine Size }\end{array}$ & Mine Name \\
\hline 144 & Hebei Province & Baoding City & Quyang County & 38.736 & 114.579 & Bituminous & Small & $\begin{array}{l}\text { Zhai li } \\
\text { Exploration Area }\end{array}$ \\
\hline 145 & Hebei Province & Shijiazhuang City & Jingxing County & 38.119 & 114.007 & Bituminous & Small & Tao yuan gao cheng \\
\hline 146 & Hebei Province & Shijiazhuang City & NA & 38.087 & 114.054 & Bituminous & Medium & $\begin{array}{l}\text { Jing xing Bureau of } \\
\text { Mine, No. } 1 \\
\text { Coal Mine. }\end{array}$ \\
\hline 147 & Hebei Province & Shijiazhuang City & Jingxing County & 38.070 & 114.097 & Bituminous & Small & Zhao cun pu \\
\hline 148 & Hebei Province & Shijiazhuang City & NA & 38.066 & 114.084 & Bituminous & Small & $\begin{array}{l}\text { Jing xing Bureau of } \\
\text { Mine, No. } 2 \\
\text { Coal Mine }\end{array}$ \\
\hline 149 & Hebei Province & Shijiazhuang City & Jingxing County & 38.066 & 114.084 & Bituminous & Small & $\begin{array}{l}\text { Bei zhuang } \\
\text { Exploration Area }\end{array}$ \\
\hline 150 & Hebei Province & Shijiazhuang City & Jingxing County & 38.068 & 114.017 & Bituminous & Small & Tian hu yu Well Field \\
\hline 151 & Hebei Province & Shijiazhuang City & Jingxing County & 38.047 & 114.015 & Bituminous & Small & $\begin{array}{l}\text { Ha ma shan } \\
\text { Exploration Area }\end{array}$ \\
\hline 152 & Hebei Province & Shijiazhuang City & NA & 38.043 & 114.049 & Bituminous & Small & Xi gou \\
\hline 153 & Hebei Province & Shijiazhuang City & NA & 38.035 & 114.033 & Bituminous & Medium & $\begin{array}{l}\text { Jing xing Bureau of } \\
\text { Mine, No. } 3 \\
\text { Coal Mine }\end{array}$ \\
\hline 154 & Hebei Province & Shijiazhuang City & Jingxing County & 38.027 & 114.031 & Bituminous & Medium & Di shui zi \\
\hline 155 & Hebei Province & Shijiazhuang City & Yuanshi County & 37.828 & 114.466 & Bituminous & Large & $\begin{array}{l}\text { Yuan bei } \\
\text { Exploration Area }\end{array}$ \\
\hline 156 & Hebei Province & Shijiazhuang City & Zanhuang County & 37.587 & 114.484 & Bituminous & Small & Bai jia yao \\
\hline 157 & Hebei Province & Xingtai City & Lincheng County & 37.537 & 114.458 & Bituminous & Small & Zhu bi Well Field \\
\hline 158 & Hebei Province & Xingtai City & Lincheng County & 37.485 & 114.455 & Bituminous & Small & $\begin{array}{l}\text { Qi cun Exploration } \\
\text { Area, Nan gong }\end{array}$ \\
\hline 159 & Hebei Province & Xingtai City & Lincheng County & 37.469 & 114.449 & Bituminous & Small & $\begin{array}{l}\text { Qi cun Exploration } \\
\text { Area, Gang xing }\end{array}$ \\
\hline 160 & Hebei Province & Shijiazhuang City & Jingxing County & 37.453 & 114.492 & Bituminous & Small & $\begin{array}{l}\text { Bureau of Mine, } \\
\text { Lin cheng }\end{array}$ \\
\hline 161 & Hebei Province & Xingtai City & Lincheng County & 37.454 & 114.471 & Bituminous & Small & $\begin{array}{l}\text { Qi cun Area, Xing tai, } \\
\text { Deng di }\end{array}$ \\
\hline 162 & Hebei Province & Xingtai City & Lincheng County & 37.438 & 114.470 & Bituminous & Small & $\begin{array}{l}\text { Qi cun Area, Long } \\
\text { yao, Nan cheng cun }\end{array}$ \\
\hline
\end{tabular}


Table 1. List of coal mines in China.-Continued

[Abbreviations used: dec. deg. - decimal degrees; NA - not available or not applicable]

\begin{tabular}{|c|c|c|c|c|c|c|c|c|}
\hline $\begin{array}{l}\text { ID } \\
\text { Number }\end{array}$ & $\begin{array}{c}\text { Province, National } \\
\text { Municipality, or } \\
\text { Autonomous Region }\end{array}$ & $\begin{array}{c}\text { Prefecture, Municipality, } \\
\text { Prefecture-Level City, or } \\
\text { League }\end{array}$ & $\begin{array}{l}\text { County, District or } \\
\text { County-Level City }\end{array}$ & $\begin{array}{l}\text { Latitude } \\
\text { (dec. deg.) }\end{array}$ & $\begin{array}{l}\text { Longitude } \\
\text { (dec. deg.) }\end{array}$ & Rank & $\begin{array}{l}\text { Relative } \\
\text { Mine Size }\end{array}$ & Mine Name \\
\hline 163 & Hebei Province & Xingtai City & Lincheng County & 37.433 & 114.497 & Bituminous & Small & $\begin{array}{l}\text { Lin cheng Well } \\
\text { Field, Ren xian } \\
\text { dong shang }\end{array}$ \\
\hline 164 & Hebei Province & Xingtai City & Neiqiu County & 37.370 & 114.510 & Bituminous & Small & $\begin{array}{l}\text { Shao ming Area, } \\
\text { Ju lu qing he }\end{array}$ \\
\hline 165 & Hebei Province & Xingtai City & Longyao County & 37.366 & 114.625 & Bituminous & Small & $\begin{array}{l}\text { Yi cheng } \\
\text { Exploration Area }\end{array}$ \\
\hline 166 & Hebei Province & Xingtai City & Longyao County & 37.360 & 114.696 & Bituminous & Medium & Yao shan \\
\hline 167 & Hebei Province & Xingtai City & Longyao County & 37.363 & 114.859 & Bituminous & Medium & $\begin{array}{l}\text { Long dong } \\
\text { Reconnaissance } \\
\text { Area }\end{array}$ \\
\hline 168 & Hebei Province & Xingtai City & Neiqiu County & 37.316 & 114.481 & Bituminous & Small & Mo wo Well Field \\
\hline 169 & Hebei Province & Xingtai City & NA & 37.249 & 114.429 & Bituminous & Large & Dong pang \\
\hline 170 & Hebei Province & Xingtai City & Neiqiu County & 37.197 & 114.395 & Bituminous & Small & $\begin{array}{l}\text { Xi pang } \\
\text { Exploration Area }\end{array}$ \\
\hline 171 & Hebei Province & Xingtai City & NA & 37.175 & 114.488 & Bituminous & Medium & Xing Coal No. 2 Area \\
\hline 172 & Hebei Province & Xingtai City & NA & 37.136 & 114.502 & Bituminous & Large & $\begin{array}{l}\text { Xing North } \\
\text { Exploration Area }\end{array}$ \\
\hline 173 & Hebei Province & Xingtai City & NA & 37.065 & 114.518 & Bituminous & Medium & $\begin{array}{l}\text { Xing East } \\
\text { Exploration Area }\end{array}$ \\
\hline 174 & Hebei Province & Xingtai City & NA & 37.048 & 114.402 & Bituminous & Small & $\begin{array}{l}\text { Xian xian } \\
\text { Exploration Area }\end{array}$ \\
\hline 175 & Hebei Province & Xingtai City & NA & 36.997 & 114.446 & Bituminous & Large & Xing tai Coal Field \\
\hline 176 & Hebei Province & Xingtai City & Pingxian County & 36.948 & 114.350 & Anthracite & Small & Shi li ting \\
\hline 177 & Hebei Province & Xingtai City & Shahe City & 36.927 & 114.339 & Bituminous & Small & $\begin{array}{l}\text { Ge quan } \\
\text { Exploration Area }\end{array}$ \\
\hline 178 & Hebei Province & Xingtai City & Shahe City & 36.912 & 114.224 & Anthracite & Small & Shang guan Well Field \\
\hline 179 & Hebei Province & Xingtai City & Shahe City & 36.899 & 114.333 & Anthracite & Small & Xu zhuang \\
\hline 180 & Hebei Province & Xingtai City & Shahe City & 36.884 & 114.251 & Anthracite & Small & Li jia zhuang \\
\hline 181 & Hebei Province & Xingtai City & Shahe City & 36.871 & 114.236 & Anthracite & Small & Xian de wang \\
\hline 182 & Hebei Province & Xingtai City & Shahe City & 36.871 & 114.318 & Anthracite & Small & Ma zhuang Well Field \\
\hline 183 & Hebei Province & Xingtai City & Shahe City & 36.874 & 114.333 & Anthracite & Small & Sna wang cun \\
\hline 184 & Hebei Province & Xingtai City & Shahe City & 36.859 & 114.313 & Anthracite & Small & Zhang cun Well Field \\
\hline 185 & Hebei Province & Xingtai City & Shahe City & 36.864 & 114.382 & Anthracite & Small & $\begin{array}{l}\text { Bei zhang } \\
\text { Exploration Area }\end{array}$ \\
\hline 186 & Hebei Province & Xingtai City & Shahe City & 36.864 & 114.216 & Anthracite & Small & Luan xie \\
\hline 187 & Hebei Province & Handan City & Wuan City & 36.844 & 114.255 & Anthracite & Small & Yi cheng Well Field \\
\hline
\end{tabular}


Table 1. List of coal mines in China.-Continued

[Abbreviations used: dec. deg. - decimal degrees; NA - not available or not applicable]

\begin{tabular}{|c|c|c|c|c|c|c|c|c|}
\hline $\begin{array}{c}\text { ID } \\
\text { Number }\end{array}$ & $\begin{array}{c}\text { Province, National } \\
\text { Municipality, or } \\
\text { Autonomous Region }\end{array}$ & $\begin{array}{c}\text { Prefecture, Municipality, } \\
\text { Prefecture-Level City, or } \\
\text { League }\end{array}$ & $\begin{array}{l}\text { County, District or } \\
\text { County-Level City }\end{array}$ & $\begin{array}{l}\text { Latitude } \\
\text { (dec. deg.) }\end{array}$ & $\begin{array}{l}\text { Longitude } \\
\text { (dec. deg.) }\end{array}$ & Rank & $\begin{array}{l}\text { Relative } \\
\text { Mine Size }\end{array}$ & Mine Name \\
\hline 188 & Hebei Province & Xingtai City & Shahe City & 36.845 & 114.336 & Anthracite & Small & $\begin{array}{l}\text { Bei zhuang Coal Field, } \\
\text { Yong nian Shang } \\
\text { zhuang ling } \\
\text { Sampling Area }\end{array}$ \\
\hline 189 & Hebei Province & Handan City & Wuan City & 36.802 & 114.201 & Anthracite & Small & $\begin{array}{l}\text { Guo er zhuang Coal } \\
\text { Mine, No. } 2 \text { Pit }\end{array}$ \\
\hline 190 & Hebei Province & Handan City & Wuan City & 36.802 & 114.201 & Anthracite & Small & $\begin{array}{l}\text { Guo er zhuang Coal } \\
\text { Mine, No. } 1 \text { Pit }\end{array}$ \\
\hline 191 & Hebei Province & Handan City & Wuan City & 36.722 & 113.940 & Bituminous & Small & Yan yi \\
\hline 192 & Hebei Province & Handan City & Wuan City & 36.735 & 114.135 & Anthracite & Medium & $\begin{array}{l}\text { Yun jia ling } \\
\text { Well Field }\end{array}$ \\
\hline 193 & Hebei Province & Handan City & Wuan City & 36.699 & 114.268 & Anthracite & Medium & $\begin{array}{l}\text { Kang er cheng Coal } \\
\text { Mine, No. } 1 \text { Pit }\end{array}$ \\
\hline 194 & Hebei Province & Handan City & NA & 36.677 & 114.300 & Anthracite & Medium & Tao er Coal Mine \\
\hline 195 & Hebei Province & Handan City & NA & 36.676 & 114.314 & Anthracite & Small & $\begin{array}{l}\text { Tao zhuang No. } 2 \\
\text { Well Field, } \\
\text { Yong nian jiao yao }\end{array}$ \\
\hline 196 & Hebei Province & Handan City & Wuan City & 36.647 & 114.115 & Anthracite & Small & He zhuang \\
\hline 197 & Hebei Province & Handan City & Wuan City & 36.649 & 114.229 & Anthracite & Medium & $\begin{array}{l}\text { Zhou zhuang Well } \\
\text { Field, Shen bu }\end{array}$ \\
\hline 198 & Hebei Province & Handan City & Wuan City & 36.653 & 114.276 & Anthracite & Small & $\begin{array}{l}\text { Kang er cheng Well } \\
\text { Field, Qiu xian } \\
\text { Sampling Area }\end{array}$ \\
\hline 199 & Hebei Province & Handan City & Wuan City & 36.651 & 114.288 & Anthracite & Medium & $\begin{array}{l}\text { Kang er chang Coal } \\
\text { Mine, No. } 3 \text { Pit }\end{array}$ \\
\hline 200 & Hebei Province & Handan City & NA & 36.647 & 114.301 & Anthracite & Medium & Tao yi Coal Mine \\
\hline 201 & Hebei Province & Handan City & Wuan City & 36.629 & 114.219 & Anthracite & Small & $\begin{array}{l}\text { Zhou zhuang Well } \\
\text { Field, Lin zhang } \\
\text { Sampling Area }\end{array}$ \\
\hline 202 & Hebei Province & Handan City & Wuan City & 36.623 & 114.211 & Anthracite & Small & $\begin{array}{l}\text { Zhou zhuang Well } \\
\text { Field, Cheng an } \\
\text { Sampling Area }\end{array}$ \\
\hline 203 & Hebei Province & Handan City & Wuan City & 36.623 & 114.228 & Anthracite & Small & $\begin{array}{l}\text { Zhou zhuang Well } \\
\text { Field }\end{array}$ \\
\hline 205 & Hebei Province & Handan City & NA & 36.632 & 114.286 & Anthracite & Small & $\begin{array}{l}\text { Tao zhuang No. } 1 \\
\text { Well Field, Heng } \\
\text { shui Sampling Area }\end{array}$ \\
\hline
\end{tabular}


Table 1. List of coal mines in China.-Continued

[Abbreviations used: dec. deg. - decimal degrees; NA - not available or not applicable]

\begin{tabular}{|c|c|c|c|c|c|c|c|c|}
\hline $\begin{array}{c}\text { ID } \\
\text { Number }\end{array}$ & $\begin{array}{c}\text { Province, National } \\
\text { Municipality, or } \\
\text { Autonomous Region }\end{array}$ & $\begin{array}{c}\text { Prefecture, Municipality, } \\
\text { Prefecture-Level City, or } \\
\text { League }\end{array}$ & $\begin{array}{l}\text { County, District or } \\
\text { County-Level City }\end{array}$ & $\begin{array}{l}\text { Latitude } \\
\text { (dec. deg.) }\end{array}$ & $\begin{array}{l}\text { Longitude } \\
\text { (dec. deg.) }\end{array}$ & Rank & $\begin{array}{l}\text { Relative } \\
\text { Mine Size }\end{array}$ & Mine Name \\
\hline 206 & Hebei Province & Handan City & NA & 36.627 & 114.297 & Anthracite & Small & $\begin{array}{l}\text { Tao zhuang No. } 1 \\
\text { Well Field, Ji ze } \\
\text { Sampling Area }\end{array}$ \\
\hline 207 & Hebei Province & Handan City & Wuan City & 36.626 & 114.273 & Anthracite & Small & $\begin{array}{l}\text { Ling shan Coal Field, } \\
\text { Qu zhou }\end{array}$ \\
\hline 208 & Hebei Province & Handan City & NA & 36.606 & 114.273 & Anthracite & Small & $\begin{array}{l}\text { Tao zhuang No. } 2 \\
\text { Well Field, } \\
\text { Ji zhuang } \\
\text { Sampling Area }\end{array}$ \\
\hline 209 & Hebei Province & Handan City & NA & 36.600 & 114.154 & Anthracite & Large & $\begin{array}{l}\text { Feng feng Bureau } \\
\text { of Mine, } \\
\text { Wan nian Mine }\end{array}$ \\
\hline 210 & Hebei Province & Handan City & NA & 36.562 & 114.237 & Anthracite & Large & $\begin{array}{l}\text { Feng feng Bureau of } \\
\text { Mine, Da jiao cun } \\
\text { Well Field }\end{array}$ \\
\hline 211 & Hebei Province & Handan City & NA & 36.546 & 114.098 & Bituminous & Medium & $\begin{array}{l}\text { Feng feng Bureau of } \\
\text { Mine, No. } 4 \text { Mine }\end{array}$ \\
\hline 212 & Hebei Province & Handan City & NA & 36.538 & 114.103 & Bituminous & Large & $\begin{array}{l}\text { Feng feng Bureau of } \\
\text { Mine, Tong er Mine }\end{array}$ \\
\hline 213 & Hebei Province & Handan City & NA & 36.548 & 114.230 & Bituminous & Large & $\begin{array}{l}\text { Feng feng Bureau of } \\
\text { Mine, Xue cun }\end{array}$ \\
\hline 214 & Hebei Province & Handan City & NA & 36.532 & 114.235 & Bituminous & Medium & $\begin{array}{l}\text { Feng feng Bureau } \\
\text { of Mine, } \\
\text { Xiao tun Mine }\end{array}$ \\
\hline 215 & Hebei Province & Handan City & NA & 36.528 & 114.219 & Bituminous & Small & $\begin{array}{l}\text { Feng feng Bureau } \\
\text { of Mine, } \\
\text { Niu er Zhuang }\end{array}$ \\
\hline 216 & Hebei Province & Handan City & NA & 36.508 & 114.268 & Bituminous & Medium & $\begin{array}{l}\text { Feng feng Bureau of } \\
\text { Mine, Yang dong } \\
\text { Well Field }\end{array}$ \\
\hline 217 & Hebei Province & Handan City & NA & 36.490 & 114.148 & Bituminous & Small & $\begin{array}{l}\text { Yi jing Coal Mine, } \\
\text { No. } 1 \text { Pit }\end{array}$ \\
\hline 218 & Hebei Province & Handan City & NA & 36.490 & 114.202 & Bituminous & Medium & $\begin{array}{l}\text { Feng feng Bureau of } \\
\text { Mine, No. } 5 \text { Mine }\end{array}$ \\
\hline 219 & Hebei Province & Handan City & NA & 36.487 & 114.233 & Bituminous & Large & $\begin{array}{l}\text { Feng feng Bureau of } \\
\text { Mine, Yang qu he }\end{array}$ \\
\hline 220 & Hebei Province & Handan City & NA & 36.470 & 114.204 & Bituminous & Medium & $\begin{array}{l}\text { Feng feng Bureau of } \\
\text { Mine, No. } 2 \text { Mine }\end{array}$ \\
\hline
\end{tabular}


Table 1. List of coal mines in China.-Continued

[Abbreviations used: dec. deg. - decimal degrees; NA - not available or not applicable]

\begin{tabular}{|c|c|c|c|c|c|c|c|c|}
\hline $\begin{array}{c}\text { ID } \\
\text { Number }\end{array}$ & $\begin{array}{c}\text { Province, National } \\
\text { Municipality, or } \\
\text { Autonomous Region }\end{array}$ & $\begin{array}{c}\text { Prefecture, Municipality, } \\
\text { Prefecture-Level City, or } \\
\text { League }\end{array}$ & $\begin{array}{l}\text { County, District or } \\
\text { County-Level City }\end{array}$ & $\begin{array}{l}\text { Latitude } \\
\text { (dec. deg.) }\end{array}$ & $\begin{array}{l}\text { Longitude } \\
\text { (dec. deg.) }\end{array}$ & Rank & $\begin{array}{l}\text { Relative } \\
\text { Mine Size }\end{array}$ & Mine Name \\
\hline 221 & Hebei Province & Handan City & NA & 36.456 & 114.091 & Bituminous & Small & $\begin{array}{l}\text { Wang feng Coal Mine, } \\
\text { No. } 1 \text { Pit }\end{array}$ \\
\hline 222 & Hebei Province & Handan City & NA & 36.458 & 114.101 & Bituminous & Medium & $\begin{array}{l}\text { Wang feng Coal Mine, } \\
\text { No. } 2 \text { Pit }\end{array}$ \\
\hline 223 & Hebei Province & Handan City & NA & 36.448 & 114.139 & Bituminous & Small & $\begin{array}{l}\text { Yi jing Coal Mine, } \\
\text { No. } 2 \text { Pit }\end{array}$ \\
\hline 224 & Hebei Province & Handan City & NA & 36.448 & 114.194 & Bituminous & Small & $\begin{array}{l}\text { Feng feng Bureau of } \\
\text { Mine, No. } 1 \text { Pit }\end{array}$ \\
\hline 225 & Hebei Province & Handan City & NA & 36.448 & 114.232 & Bituminous & Small & $\begin{array}{l}\text { Feng feng Bureau of } \\
\text { Mine, Jiu long kou, } \\
\text { Pu zi cun Well Field }\end{array}$ \\
\hline 226 & Hebei Province & Handan City & NA & 36.425 & 114.129 & Bituminous & Small & Sha guo yuan \\
\hline 227 & Hebei Province & Handan City & Cixian County & 36.417 & 114.054 & Bituminous & Small & Bai tu \\
\hline 228 & Hebei Province & Handan City & NA & 36.406 & 114.133 & Bituminous & Medium & $\begin{array}{r}\text { Feng feng Bureau of } \\
\text { Mine, Sun zhuang }\end{array}$ \\
\hline 229 & Hebei Province & Handan City & NA & 36.389 & 114.185 & Bituminous & Small & $\begin{array}{l}\text { Feng feng Bureau of } \\
\text { Mine, Northern } \\
\text { No. } 3 \text { Mine, Da yu } \\
\text { Well Field }\end{array}$ \\
\hline 230 & Hebei Province & Handan City & Cixian County & 36.386 & 114.072 & Bituminous & Small & $\begin{array}{l}\text { Du dang Coal Mine, } \\
\text { No. } 1 \text { Pit }\end{array}$ \\
\hline 231 & Hebei Province & Handan City & Cixian County & 36.386 & 114.089 & Bituminous & Small & $\begin{array}{l}\text { Du dang Coal Mine, } \\
\text { No. } 2 \text { Pit }\end{array}$ \\
\hline 232 & Hebei Province & Handan City & Cixian County & 36.381 & 114.076 & Bituminous & Small & $\begin{array}{l}\text { Du dang Coal Mine, } \\
\text { Xin zhuang } \\
\text { Well Field }\end{array}$ \\
\hline 233 & Hebei Province & Handan City & NA & 36.381 & 114.127 & Bituminous & Medium & $\begin{array}{l}\text { Feng feng Bureau of } \\
\text { Mine, Huang sha }\end{array}$ \\
\hline 234 & Hebei Province & Handan City & Cixian County & 36.362 & 114.114 & Bituminous & Small & $\begin{array}{l}\text { Shen jia zhuang } \\
\text { Well Field }\end{array}$ \\
\hline 235 & Hebei Province & Handan City & NA & 36.360 & 114.170 & Bituminous & Large & $\begin{array}{l}\text { Feng feng Bureau of } \\
\text { Mine, No. } 3 \text { Mine }\end{array}$ \\
\hline 236 & Hebei Province & Handan City & NA & 36.355 & 114.186 & Bituminous & Medium & $\begin{array}{l}\text { Wu tong zhuang } \\
\text { Well Field }\end{array}$ \\
\hline 237 & Hebei Province & Handan City & NA & 36.353 & 114.174 & Bituminous & Medium & $\begin{array}{l}\text { Feng feng Bureau of } \\
\text { Mine, Eastern } \\
\text { Wu tong zhuang } \\
\text { Well Field }\end{array}$ \\
\hline
\end{tabular}


Table 1. List of coal mines in China.-Continued

[Abbreviations used: dec. deg. - decimal degrees; NA - not available or not applicable]

\begin{tabular}{|c|c|c|c|c|c|c|c|c|}
\hline $\begin{array}{c}\text { ID } \\
\text { Number }\end{array}$ & $\begin{array}{c}\text { Province, National } \\
\text { Municipality, or } \\
\text { Autonomous Region }\end{array}$ & $\begin{array}{c}\text { Prefecture, Municipality, } \\
\text { Prefecture-Level City, or } \\
\text { League }\end{array}$ & $\begin{array}{l}\text { County, District or } \\
\text { County-Level City }\end{array}$ & $\begin{array}{l}\text { Latitude } \\
\text { (dec. deg.) }\end{array}$ & $\begin{array}{l}\text { Longitude } \\
\text { (dec. deg.) }\end{array}$ & Rank & $\begin{array}{l}\text { Relative } \\
\text { Mine Size }\end{array}$ & Mine Name \\
\hline 238 & Hebei Province & Handan City & Cixian County & 36.298 & 114.078 & Bituminous & Small & Guan tai \\
\hline 239 & Hebei Province & Handan City & Cixian County & 36.291 & 114.122 & Bituminous & Small & Xi nan Well Field \\
\hline 2 & Heilongjiang Province & Da Hinggan Ling City & Mohe County & 53.156 & 122.049 & Bituminous & Small & Huo la pen \\
\hline 7 & Heilongjiang Province & Heihe City & Nenjiang County & 50.139 & 125.786 & Bituminous & Small & Hei bao shan \\
\hline 9 & Heilongjiang Province & Heihe City & NA & 50.027 & 127.359 & Lignite & Small & $\begin{array}{l}\text { Xi gang zi San dao } \\
\text { gou Reconaissance } \\
\text { Survey Area }\end{array}$ \\
\hline 11 & Heilongjiang Province & Heihe City & NA & 49.939 & 127.315 & Lignite & Small & $\begin{array}{l}\text { Xi gang zi Coal Mine, } \\
\text { San ji tun Area }\end{array}$ \\
\hline 19 & Heilongjiang Province & Harbin City & Yilan County & 46.132 & 129.471 & Bituminous & Small & \\
\hline 21 & Heilongjiang Province & Hegang City & Suibin County & 47.509 & 131.672 & Bituminous & Medium & $\begin{array}{c}\text { Sui bin Coal Mine } \\
\text { Reconnaissance } \\
\text { Survey Area }\end{array}$ \\
\hline 22 & Heilongjiang Province & Hegang City & NA & 47.348 & 130.504 & Lignite & Small & $\begin{array}{l}\text { He gang Eastern } \\
\text { Coal Mine } \\
\text { Reconnaissance } \\
\text { Survey Area }\end{array}$ \\
\hline 23 & Heilongjiang Province & Hegang City & NA & 47.256 & 130.274 & Bituminous & Large & \\
\hline 24 & Heilongjiang Province & Shuangyashan City & Jixian County & 46.972 & 131.538 & Bituminous & Large & $\begin{array}{l}\text { Dong rong Accurate } \\
\text { Survey } \\
\text { Reconnaissance } \\
\text { Survey Area }\end{array}$ \\
\hline 26 & Heilongjiang Province & Shuangyashan City & NA & 46.532 & 131.309 & Bituminous & Large & \\
\hline 27 & Heilongjiang Province & Qitaihe City & Boli County & 46.066 & 131.348 & Bituminous & Small & $\begin{array}{l}\text { Bei xing } \\
\quad \text { Exploration Area }\end{array}$ \\
\hline 34 & Heilongjiang Province & Qitaihe City & NA & 45.944 & 131.272 & Bituminous & Large & \\
\hline 35 & Heilongjiang Province & Jixi City & NA & 45.520 & 130.958 & Bituminous & Small & $\begin{array}{r}\text { Si hai, outskirts of } \\
\text { Coal Mine Area }\end{array}$ \\
\hline 36 & Heilongjiang Province & Jixi City & NA & 45.194 & 130.679 & Bituminous & Small & $\begin{array}{l}\text { Ping ma North } \\
\text { Reconnaissance } \\
\text { Survey Area }\end{array}$ \\
\hline 37 & Heilongjiang Province & Jixi City & NA & 45.269 & 130.858 & Bituminous & Large & \\
\hline 38 & Heilongjiang Province & Jixi City & Jidong County & 45.199 & 131.027 & Bituminous & Small & \\
\hline 40 & Heilongjiang Province & Mudanjiang City & Muling County & 44.996 & 130.877 & Lignite & Medium & $\begin{array}{l}\text { Ba mian tong, } \\
\text { Huo shao qiao } \\
\text { Reconnaissance } \\
\text { Survey Area }\end{array}$ \\
\hline
\end{tabular}


Table 1. List of coal mines in China. - Continued

[Abbreviations used: dec. deg. - decimal degrees; NA - not available or not applicable]

\begin{tabular}{|c|c|c|c|c|c|c|c|c|}
\hline $\begin{array}{c}\text { ID } \\
\text { Number }\end{array}$ & $\begin{array}{c}\text { Province, National } \\
\text { Municipality, or } \\
\text { Autonomous Region }\end{array}$ & $\begin{array}{c}\text { Prefecture, Municipality, } \\
\text { Prefecture-Level City, or } \\
\text { League }\end{array}$ & $\begin{array}{l}\text { County, District or } \\
\text { County-Level City }\end{array}$ & $\begin{array}{l}\text { Latitude } \\
\text { (dec. deg.) }\end{array}$ & $\begin{array}{l}\text { Longitude } \\
\text { (dec. deg.) }\end{array}$ & Rank & $\begin{array}{l}\text { Relative } \\
\text { Mine Size }\end{array}$ & Mine Name \\
\hline 42 & Heilongjiang Province & Qitaihe City & NA & 45.968 & 132.078 & Bituminous & Small & $\begin{array}{l}\text { Shi ba li } \\
\text { Reconnaissance } \\
\text { Survey Area }\end{array}$ \\
\hline 43 & Heilongjiang Province & Jixi City & Hulin County & 45.669 & 132.518 & Lignite & Small & $\begin{array}{l}\text { Da qing shan Deposit } \\
\text { Searching } \\
\text { Reconnaissance } \\
\text { Survey Area }\end{array}$ \\
\hline 46 & Heilongjiang Province & Mudanjiang City & Dongning County & 43.666 & 130.936 & Bituminous & Small & $\begin{array}{l}\text { Lao hei shan } \\
\text { Reconnaissance } \\
\text { Survey Area } \\
\end{array}$ \\
\hline 1 & Henan Province & Anyang City & NA & 36.271 & 114.072 & Bituminous & Medium & Gang zi yao \\
\hline 2 & Henan Province & Anyang City & NA & 36.263 & 114.124 & Bituminous & Small & Hong ling \\
\hline 3 & Henan Province & Anyang City & NA & 36.195 & 114.105 & Anthracite & Large & Tong ye \\
\hline 4 & Henan Province & Anyang City & NA & 36.161 & 114.088 & Anthracite & Medium & Ban Mine \\
\hline 5 & Henan Province & Anyang City & NA & 36.095 & 114.170 & Anthracite & Medium & Long shan \\
\hline 6 & Henan Province & Hebi City & NA & 36.020 & 114.168 & Bituminous & Medium & Long gong \\
\hline 7 & Henan Province & Puyang City & NA & 36.010 & 114.150 & Bituminous & Medium & Jiu Mine \\
\hline 8 & Henan Province & Puyang City & Taiqian County & 36.047 & 116.049 & Bituminous & Medium & Wu ba \\
\hline 9 & Henan Province & Jiyuan City & NA & 35.166 & 112.548 & Anthracite & Medium & Ren zhuang \\
\hline 10 & Henan Province & Jiyuan City & NA & 35.091 & 112.074 & Bituminous & Small & Jiang ge da \\
\hline 11 & Henan Province & Jiyuan City & NA & 34.998 & 112.247 & Bituminous & Medium & Xia ye \\
\hline 12 & Henan Province & Xinxiang City & Huixian City & 35.500 & 113.910 & Anthracite & Small & Zhang cun \\
\hline 13 & Henan Province & Xinxiang City & Huixian City & 35.423 & 113.645 & Anthracite & Large & Zhao gu \\
\hline 14 & Henan Province & Jiaozuo City & Xiuwu City & 35.364 & 113.424 & Anthracite & Medium & Fang zhuang \\
\hline 15 & Henan Province & Xinxiang City & Huixian City & 35.357 & 113.477 & Anthracite & Small & Wu cun \\
\hline 16 & Henan Province & Jiaozuo City & Xiuwu City & 35.350 & 113.446 & Anthracite & Small & Bai zhuang \\
\hline 17 & Henan Province & Jiaozuo City & NA & 35.345 & 113.478 & Anthracite & Medium & Gu han shan \\
\hline 18 & Henan Province & Jiaozuo City & NA & 35.337 & 113.402 & Anthracite & Medium & Feng ying \\
\hline 19 & Henan Province & Jiaozuo City & NA & 35.316 & 113.420 & Anthracite & Medium & Jiu li shan \\
\hline 20 & Henan Province & Jiaozuo City & NA & 35.278 & 113.423 & Anthracite & Small & Ma fang quan \\
\hline 21 & Henan Province & Jiaozuo City & NA & 35.270 & 113.372 & Anthracite & Medium & Yan ma zhuang \\
\hline 22 & Henan Province & Jiaozuo City & NA & 35.306 & 113.343 & Anthracite & Small & Tian men \\
\hline 23 & Henan Province & Jiaozuo City & NA & 35.284 & 113.326 & Anthracite & Small & Xiao ma cun \\
\hline 24 & Henan Province & Jiaozuo City & NA & 35.262 & 113.339 & Anthracite & Small & Han wang \\
\hline 25 & Henan Province & Jiaozuo City & NA & 35.259 & 113.308 & Anthracite & Medium & Zhong ma cun \\
\hline 26 & Henan Province & Jiaozuo City & NA & 35.246 & 113.172 & Anthracite & Small & Ta zhang \\
\hline 27 & Henan Province & Jiaozuo City & NA & 35.235 & 113.174 & Anthracite & Small & Li feng \\
\hline 28 & Henan Province & Jiaozuo City & NA & 35.233 & 113.155 & Anthracite & Medium & Wang feng \\
\hline 29 & Henan Province & Jiaozuo City & NA & 35.230 & 113.129 & Anthracite & Small & Min you \\
\hline
\end{tabular}


Table 1. List of coal mines in China. - Continued

[Abbreviations used: dec. deg. - decimal degrees; NA - not available or not applicable]

\begin{tabular}{|c|c|c|c|c|c|c|c|c|}
\hline $\begin{array}{c}\text { ID } \\
\text { Number }\end{array}$ & $\begin{array}{l}\text { Province, National } \\
\text { Municipality, or } \\
\text { Autonomous Region }\end{array}$ & $\begin{array}{c}\text { Prefecture, Municipality, } \\
\text { Prefecture-Level City, or } \\
\text { League }\end{array}$ & $\begin{array}{l}\text { County, District or } \\
\text { County-Level City }\end{array}$ & $\begin{array}{l}\text { Latitude } \\
\text { (dec. deg.) }\end{array}$ & $\begin{array}{l}\text { Longitude } \\
\text { (dec. deg.) }\end{array}$ & Rank & $\begin{array}{l}\text { Relative } \\
\text { Mine Size }\end{array}$ & Mine Name \\
\hline 30 & Henan Province & Jiaozuo City & NA & 35.231 & 113.271 & Anthracite & Large & Si cun \\
\hline 31 & Henan Province & Jiaozuo City & NA & 35.231 & 113.304 & Anthracite & Large & Qiang nan \\
\hline 32 & Henan Province & Jiaozuo City & NA & 35.254 & 113.251 & Anthracite & Small & Jiao dong \\
\hline 33 & Henan Province & Jiaozuo City & NA & 35.229 & 113.209 & Anthracite & Small & Jiao xi \\
\hline 34 & Henan Province & Jiaozuo City & NA & 35.210 & 113.175 & Anthracite & Medium & Zhu cun \\
\hline 35 & Henan Province & Jiaozuo City & NA & 35.202 & 113.227 & Anthracite & Medium & Jiao nan \\
\hline 36 & Henan Province & Hebi City & NA & 35.937 & 114.161 & Bituminous & Medium & No. 2 Mine \\
\hline 37 & Henan Province & Hebi City & NA & 35.923 & 114.220 & Bituminous & Large & Outer skirt \\
\hline 38 & Henan Province & Hebi City & NA & 35.867 & 114.211 & Bituminous & Medium & No. 6 Mine \\
\hline 39 & Henan Province & Hebi City & NA & 35.818 & 114.204 & Bituminous & Medium & Ba sha Eastern Well \\
\hline 40 & Henan Province & Sanmenxia City & Mianchi County & 34.819 & 111.661 & Bituminous & Small & Chen cun \\
\hline 41 & Henan Province & Sanmenxia City & Mianchi County & 34.818 & 111.808 & Bituminous & Medium & Ren cun Du jia \\
\hline 42 & Henan Province & Sanmenxia City & Shan County & 34.797 & 111.480 & Bituminous & Small & Qing shui he \\
\hline 43 & Henan Province & Sanmenxia City & Shan County & 34.793 & 111.554 & Bituminous & Medium & Lai ao \\
\hline 44 & Henan Province & Sanmenxia City & Mianchi County & 34.756 & 111.581 & Bituminous & Medium & Long wang zhuang \\
\hline 45 & Henan Province & Sanmenxia City & Shan County & 34.728 & 111.541 & Bituminous & Medium & Gan hao \\
\hline 46 & Henan Province & Sanmenxia City & Mianchi County & 34.726 & 111.809 & Bituminous & Large & Geng cun \\
\hline 47 & Henan Province & Sanmenxia City & Yima City & 34.718 & 111.883 & Bituminous & Large & Qian qiu \\
\hline 48 & Henan Province & Sanmenxia City & Mianchi County & 34.703 & 111.751 & Bituminous & Medium & Yang cun \\
\hline 49 & Henan Province & Sanmenxia City & Yima City & 34.691 & 111.915 & Bituminous & Medium & Chang cun \\
\hline 51 & Henan Province & Luoyang City & Xinan County & 34.929 & 112.160 & Bituminous & Small & Kuang kou \\
\hline 52 & Henan Province & Luoyang City & Xinan County & 34.897 & 112.229 & Bituminous & Small & Juan zhuang \\
\hline 53 & Henan Province & Luoyang City & Xinan County & 34.857 & 112.135 & Bituminous & Medium & Bei ye \\
\hline 54 & Henan Province & Luoyang City & Xinan County & 34.801 & 112.134 & Bituminous & Large & Xi nan \\
\hline 55 & Henan Province & Luoyang City & Xinan County & 34.749 & 112.043 & Bituminous & Small & Qu li \\
\hline 56 & Henan Province & Luoyang City & Xinan County & 34.688 & 112.095 & Bituminous & Small & Yu shan \\
\hline 57 & Henan Province & Zhengzhou City & Gongyi County & 34.595 & 112.945 & Anthracite & Medium & Yao ling \\
\hline 58 & Henan Province & Luoyang City & Yanshi City & 34.562 & 112.632 & Anthracite & Medium & Pi an qiao \\
\hline 59 & Henan Province & Luoyang City & NA & 34.561 & 112.477 & Anthracite & Small & Long men \\
\hline 60 & Henan Province & Luoyang City & Yanshi City & 34.558 & 112.750 & Anthracite & Medium & Guo cun \\
\hline 61 & Henan Province & Luoyang City & Yanshi City & 34.559 & 112.853 & Anthracite & Medium & Song shan \\
\hline 62 & Henan Province & Luoyang City & Yiyang County & 34.502 & 112.178 & Bituminous & Small & Shen cun \\
\hline 63 & Henan Province & Luoyang City & Yiyang County & 34.485 & 112.239 & Bituminous & Small & Sha po \\
\hline 64 & Henan Province & Luoyang City & Yichuan County & 34.419 & 112.283 & Bituminous & Small & Gao shan \\
\hline 65 & Henan Province & Luoyang City & Yichuan County & 34.412 & 112.211 & Anthracite & Small & $\mathrm{Xi}$ ma cun \\
\hline 66 & Henan Province & Zhengzhou City & Dengfeng City & 34.364 & 112.910 & Bituminous & Medium & Guo gou \\
\hline 67 & Henan Province & Zhengzhou City & Dengfeng City & 34.362 & 112.756 & Bituminous & Medium & Ma ling shan \\
\hline 68 & Henan Province & Zhengzhou City & Dengfeng City & 34.353 & 112.980 & Bituminous & Medium & Xin xin \\
\hline 69 & Henan Province & Zhengzhou City & Dengfeng City & 34.340 & 112.639 & Bituminous & Medium & Bao yu shan \\
\hline
\end{tabular}


Table 1. List of coal mines in China. - Continued

[Abbreviations used: dec. deg. - decimal degrees; NA - not available or not applicable]

\begin{tabular}{|c|c|c|c|c|c|c|c|c|}
\hline $\begin{array}{c}\text { ID } \\
\text { Number }\end{array}$ & $\begin{array}{c}\text { Province, National } \\
\text { Municipality, or } \\
\text { Autonomous Region }\end{array}$ & $\begin{array}{c}\text { Prefecture, Municipality, } \\
\text { Prefecture-Level City, or } \\
\text { League }\end{array}$ & $\begin{array}{l}\text { County, District or } \\
\text { County-Level City }\end{array}$ & $\begin{array}{l}\text { Latitude } \\
\text { (dec. deg.) }\end{array}$ & $\begin{array}{l}\text { Longitude } \\
\text { (dec. deg.) }\end{array}$ & Rank & $\begin{array}{l}\text { Relative } \\
\text { Mine Size }\end{array}$ & Mine Name \\
\hline 70 & Henan Province & Pingdingshan City & Ruzhou City & 34.251 & 112.809 & Bituminous & Medium & Bi shan No. 2 Well \\
\hline 71 & Henan Province & Luoyang City & Ruzhou City & 34.124 & 112.465 & Bituminous & Small & Cheng dong \\
\hline 72 & Henan Province & Pingdingshan City & Ruzhou City & 34.083 & 112.640 & Bituminous & Small & San li zhai No. 1 Well \\
\hline 73 & Henan Province & Pingdingshan City & Ruzhou City & 34.063 & 112.874 & Bituminous & Medium & Guo zhuang \\
\hline 74 & Henan Province & Pingdingshan City & Ruzhou City & 34.060 & 112.744 & Bituminous & Small & Ren cun \\
\hline 75 & Henan Province & Pingdingshan City & Ruzhou City & 34.036 & 112.861 & Bituminous & Medium & Zhang cun \\
\hline 76 & Henan Province & Zhengzhou City & Gongyi County & 34.732 & 113.070 & Anthracite & Medium & Da yu gou \\
\hline 77 & Henan Province & Zhengzhou City & Xingyang City & 34.714 & 113.314 & Anthracite & Medium & Xu zhuang \\
\hline 78 & Henan Province & Zhengzhou City & Gongyi County & 34.707 & 113.184 & Anthracite & Medium & Gu shan \\
\hline 79 & Henan Province & Zhengzhou City & Xingyang City & 34.622 & 113.535 & Anthracite & Large & San li \\
\hline 80 & Henan Province & Zhengzhou City & Gongyi County & 34.618 & 113.032 & Anthracite & Medium & Shang zhuang \\
\hline 81 & Henan Province & Zhengzhou City & $\begin{array}{l}\text { Xinmi City } \\
\text { (formerly Mi County) }\end{array}$ & 34.556 & 113.512 & Bituminous & Small & Wang zhai he \\
\hline 82 & Henan Province & Zhengzhou City & $\begin{array}{l}\text { Xinmi City } \\
\text { (formerly Mi County) }\end{array}$ & 34.541 & 113.510 & Anthracite & Medium & Lu gou \\
\hline 83 & Henan Province & Zhengzhou City & $\begin{array}{l}\text { Xinmi City } \\
\text { (formerly Mi County) }\end{array}$ & 34.535 & 113.606 & Anthracite & Large & Qu liang \\
\hline 84 & Henan Province & Zhengzhou City & $\begin{array}{l}\text { Xinmi City } \\
\text { (formerly Mi County) }\end{array}$ & 34.526 & 113.506 & Bituminous & Small & Ren gang \\
\hline 85 & Henan Province & Zhengzhou City & $\begin{array}{l}\text { Xinmi City } \\
\text { (formerly Mi County) }\end{array}$ & 34.535 & 113.294 & Anthracite & Medium & Mi cun \\
\hline 86 & Henan Province & Zhengzhou City & $\begin{array}{l}\text { Xinmi City } \\
\text { (formerly Mi County) }\end{array}$ & 34.532 & 113.245 & Anthracite & Medium & Wang zhuang \\
\hline 87 & Henan Province & Zhengzhou City & $\begin{array}{l}\text { Xinmi City } \\
\text { (formerly Mi County) }\end{array}$ & 34.503 & 113.317 & Anthracite & Small & Mi cun Niu dian \\
\hline 88 & Henan Province & Zhengzhou City & $\begin{array}{l}\text { Xinmi City } \\
\text { (formerly Mi County) }\end{array}$ & 34.502 & 113.398 & Bituminous & Small & Liang gou \\
\hline 89 & Henan Province & Zhengzhou City & $\begin{array}{l}\text { Xinmi City } \\
\text { (formerly Mi County) }\end{array}$ & 34.495 & 113.388 & Bituminous & Small & Wang gou \\
\hline 90 & Henan Province & Zhengzhou City & $\begin{array}{l}\text { Xinmi City } \\
\text { (formerly Mi County) }\end{array}$ & 34.494 & 113.406 & Bituminous & Small & Eastern Well Gu dong \\
\hline 91 & Henan Province & Zhengzhou City & $\begin{array}{l}\text { Xinmi City } \\
\text { (formerly Mi County) }\end{array}$ & 34.499 & 113.479 & Bituminous & Small & Yang jia wa \\
\hline 92 & Henan Province & Zhengzhou City & $\begin{array}{l}\text { Xinmi City } \\
\text { (formerly Mi County) }\end{array}$ & 34.487 & 113.474 & Bituminous & Medium & Pei gou \\
\hline 91 & Henan Province & Zhengzhou City & $\begin{array}{l}\text { Xinmi City } \\
\text { (formerly Mi County) }\end{array}$ & 34.499 & 113.479 & Bituminous & Small & Yang jia wa \\
\hline 92 & Henan Province & Zhengzhou City & $\begin{array}{l}\text { Xinmi City } \\
\text { (formerly Mi County) }\end{array}$ & 34.487 & 113.474 & Bituminous & Medium & Pei gou \\
\hline
\end{tabular}


Table 1. List of coal mines in China. - Continued

[Abbreviations used: dec. deg. - decimal degrees; NA - not available or not applicable]

\begin{tabular}{|c|c|c|c|c|c|c|c|c|}
\hline $\begin{array}{c}\text { ID } \\
\text { Number }\end{array}$ & $\begin{array}{c}\text { Province, National } \\
\text { Municipality, or } \\
\text { Autonomous Region }\end{array}$ & $\begin{array}{c}\text { Prefecture, Municipality, } \\
\text { Prefecture-Level City, or } \\
\text { League }\end{array}$ & $\begin{array}{l}\text { County, District or } \\
\text { County-Level City }\end{array}$ & $\begin{array}{l}\text { Latitude } \\
\text { (dec. deg.) }\end{array}$ & $\begin{array}{l}\text { Longitude } \\
\text { (dec. deg.) }\end{array}$ & Rank & $\begin{array}{l}\text { Relative } \\
\text { Mine Size }\end{array}$ & Mine Name \\
\hline 93 & Henan Province & Zhengzhou City & $\begin{array}{l}\text { Xinmi City } \\
\text { (formerly Mi County) }\end{array}$ & 34.454 & 113.503 & Bituminous & Medium & Fan zhai \\
\hline 94 & Henan Province & Zhengzhou City & Dengfeng City & 34.447 & 113.187 & Bituminous & Large & Lu dian \\
\hline 95 & Henan Province & Zhengzhou City & Dengfeng City & 34.439 & 113.232 & Bituminous & Small & Dong shi cun \\
\hline 96 & Henan Province & Zhengzhou City & $\begin{array}{l}\text { Xinmi City } \\
\text { (formerly Mi County) }\end{array}$ & 34.436 & 113.309 & Bituminous & Medium & Ping mo \\
\hline 97 & Henan Province & Zhengzhou City & Dengfeng City & 34.430 & 113.249 & Bituminous & Small & Xiao he \\
\hline 98 & Henan Province & Zhengzhou City & $\begin{array}{l}\text { Xinmi City } \\
\text { (formerly Mi County) }\end{array}$ & 34.426 & 113.351 & Bituminous & Medium & Ping mo Outskirts \\
\hline 99 & Henan Province & Zhengzhou City & $\begin{array}{l}\text { Xinmi City } \\
\text { (formerly Mi County) }\end{array}$ & 34.452 & 113.400 & Bituminous & Small & Chao hua Mine \\
\hline 100 & Henan Province & Zhengzhou City & $\begin{array}{l}\text { Xinmi City } \\
\text { (formerly Mi County) }\end{array}$ & 34.438 & 113.436 & Bituminous & Medium & Chao hua Outskirts \\
\hline 101 & Henan Province & Zhengzhou City & $\begin{array}{l}\text { Xinmi City } \\
\text { (formerly Mi County) }\end{array}$ & 34.415 & 113.398 & Bituminous & Medium & Chao hua Well Field \\
\hline 102 & Henan Province & Zhengzhou City & $\begin{array}{l}\text { Xinmi City } \\
\text { (formerly Mi County) }\end{array}$ & 34.414 & 113.489 & Bituminous & Small & Wang cun \\
\hline 103 & Henan Province & Zhengzhou City & Dengfeng City & 34.415 & 113.188 & Bituminous & Large & Zhao yang gou \\
\hline 104 & Henan Province & Zhengzhou City & $\begin{array}{l}\text { Xinmi City } \\
\text { (formerly Mi County) }\end{array}$ & 34.404 & 113.502 & Bituminous & Small & Gou tang \\
\hline 105 & Henan Province & Zhengzhou City & Xinzheng City & 34.391 & 113.638 & Bituminous & Large & Xin zheng \\
\hline 106 & Henan Province & Zhengzhou City & Dengfeng City & 34.377 & 113.136 & Bituminous & Medium & Gao cheng \\
\hline 107 & Henan Province & Zhengzhou City & Dengfeng City & 34.350 & 113.137 & Bituminous & Medium & Ji shan \\
\hline 108 & Henan Province & Zhengzhou City & Xinzheng City & 34.327 & 113.807 & Bituminous & Large & Li liang dian \\
\hline 109 & Henan Province & Xuchang City & Yuzhou City & 34.274 & 113.433 & Bituminous & Medium & $\mathrm{Pa}$ cun \\
\hline 110 & Henan Province & Xuchang City & Yuzhou City & 34.253 & 113.222 & Bituminous & Large & Fang shan \\
\hline 111 & Henan Province & Xuchang City & Yuzhou City & 34.209 & 113.514 & Bituminous & Medium & Xin feng No. 1 Mine \\
\hline 112 & Henan Province & Xuchang City & Yuzhou City & 34.167 & 113.213 & Bituminous & Medium & Yun gai shan \\
\hline 113 & Henan Province & Xuchang City & Yuzhou City & 34.153 & 113.426 & Bituminous & Medium & Yin cun fault North \\
\hline 114 & Henan Province & Xuchang City & Yuzhou City & 34.133 & 113.339 & Bituminous & Small & Xin feng No. 5 Mine \\
\hline 115 & Henan Province & Xuchang City & Yuzhou City & 34.121 & 113.291 & Bituminous & Small & Xin feng No. 2 Mine \\
\hline 116 & Henan Province & Xuchang City & Yuzhou City & 34.111 & 113.438 & Bituminous & Large & $\begin{array}{l}\text { Liang bei No. } 1 \\
\text { Well Field }\end{array}$ \\
\hline 117 & Henan Province & Xuchang City & Yuzhou City & 34.110 & 113.211 & Bituminous & Medium & Xin feng No. 6 Mine \\
\hline 118 & Henan Province & Pingdingshan City & Jia County & 34.079 & 113.158 & Bituminous & Small & Jing jia wa \\
\hline 119 & Henan Province & Xuchang City & Yuzhou City & 34.082 & 113.538 & Bituminous & Large & $\begin{array}{l}\text { Liang bei No. } 2 \\
\text { Well Field }\end{array}$ \\
\hline 120 & Henan Province & Shanqiu City & Xiayi County & 34.354 & 116.247 & Bituminous & Medium & Luo ji \\
\hline 121 & Henan Province & Shanqiu City & Yong City & 34.109 & 116.437 & Bituminous & Large & Xue hu \\
\hline
\end{tabular}


Table 1. List of coal mines in China. - Continued

[Abbreviations used: dec. deg. - decimal degrees; NA - not available or not applicable]

\begin{tabular}{|c|c|c|c|c|c|c|c|c|}
\hline $\begin{array}{c}\text { ID } \\
\text { Number }\end{array}$ & $\begin{array}{c}\text { Province, National } \\
\text { Municipality, or } \\
\text { Autonomous Region }\end{array}$ & $\begin{array}{c}\text { Prefecture, Municipality, } \\
\text { Prefecture-Level City, or } \\
\text { League }\end{array}$ & $\begin{array}{l}\text { County, District or } \\
\text { County-Level City }\end{array}$ & $\begin{array}{l}\text { Latitude } \\
\text { (dec. deg.) }\end{array}$ & $\begin{array}{l}\text { Longitude } \\
\text { (dec. deg.) }\end{array}$ & Rank & $\begin{array}{l}\text { Relative } \\
\text { Mine Size }\end{array}$ & Mine Name \\
\hline 122 & Henan Province & Pingdingshan City & NA & 33.905 & 112.828 & Bituminous & Small & $\begin{array}{l}\text { Han zhuang } \\
\text { No. } 2 \text { Mine }\end{array}$ \\
\hline 123 & Henan Province & Pingdingshan City & NA & 33.882 & 112.849 & Bituminous & Medium & Gao zhuang \\
\hline 124 & Henan Province & Pingdingshan City & NA & 33.867 & 112.847 & Bituminous & Small & Qing cao ling \\
\hline 125 & Henan Province & Pingdingshan City & NA & 33.864 & 112.857 & Bituminous & Small & Ma dao \\
\hline 126 & Henan Province & Pingdingshan City & NA & 33.851 & 112.883 & Bituminous & Medium & Da zhuang \\
\hline 127 & Henan Province & Pingdingshan City & Baofeng County & 33.849 & 112.927 & Bituminous & Small & Miao li \\
\hline 128 & Henan Province & Pingdingshan City & NA & 33.841 & 112.897 & Bituminous & Small & Jun ying gou \\
\hline 129 & Henan Province & Pingdingshan City & NA & 33.832 & 112.908 & Bituminous & Small & $\mathrm{Xu}$ fang \\
\hline 130 & Henan Province & Pingdingshan City & Lushan County & 33.823 & 112.873 & Anthracite & Small & Jin jia men \\
\hline 131 & Henan Province & Pingdingshan City & Lushan County & 33.810 & 112.873 & Anthracite & Small & Duan dian \\
\hline 132 & Henan Province & Nanyang City & Nanzhao County & 33.509 & 112.338 & Bituminous & Medium & Long tan gou \\
\hline 133 & Henan Province & Pingdingshan City & NA & 33.926 & 113.336 & Bituminous & Medium & No. 13 Mine Outskirts \\
\hline 134 & Henan Province & Pingdingshan City & NA & 33.858 & 113.399 & Bituminous & Large & No. 13 Mine \\
\hline 135 & Henan Province & Pingdingshan City & NA & 33.809 & 113.151 & Bituminous & Medium & Xiang shan \\
\hline 136 & Henan Province & Pingdingshan City & NA & 33.806 & 113.172 & Bituminous & Medium & No. 11 Mine \\
\hline 137 & Henan Province & Pingdingshan City & NA & 33.789 & 113.190 & Bituminous & Small & No. 9 Mine \\
\hline 138 & Henan Province & Xuchang City & Xiangcheng County & 33.802 & 113.485 & Bituminous & Small & Shou shan \\
\hline 139 & Henan Province & Pingdingshan City & NA & 33.773 & 113.298 & Bituminous & Large & Ping long \\
\hline 140 & Henan Province & Pingdingshan City & NA & 33.773 & 113.241 & Bituminous & Medium & No. 6 Mine \\
\hline 141 & Henan Province & Pingdingshan City & NA & 33.769 & 113.222 & Bituminous & Medium & No. 5 Mine \\
\hline 142 & Henan Province & Pingdingshan City & NA & 33.759 & 113.264 & Bituminous & Medium & No. 4 Mine \\
\hline 143 & Henan Province & Pingdingshan City & NA & 33.757 & 113.302 & Bituminous & Small & Si gou \\
\hline 144 & Henan Province & Pingdingshan City & NA & 33.746 & 113.273 & Bituminous & Small & No. 3 Mine \\
\hline 145 & Henan Province & Pingdingshan City & NA & 33.745 & 113.254 & Bituminous & Medium & No. 7 Mine \\
\hline 146 & Henan Province & Pingdingshan City & NA & 33.737 & 113.293 & Bituminous & Medium & No. 1 Mine \\
\hline 147 & Henan Province & Pingdingshan City & NA & 33.729 & 113.299 & Bituminous & Small & No. 2 Mine \\
\hline 148 & Henan Province & Pingdingshan City & NA & 33.748 & 113.368 & Bituminous & Medium & No. 12 Mine \\
\hline 149 & Henan Province & Pingdingshan City & NA & 33.740 & 113.346 & Bituminous & Medium & No. 10 Mine \\
\hline 150 & Henan Province & Pingdingshan City & NA & 33.741 & 113.424 & Bituminous & Large & \\
\hline 151 & Henan Province & Shanqiu City & Yong City & 33.980 & 116.555 & Anthracite & Medium & Hui cun \\
\hline 152 & Henan Province & Shanqiu City & Yong City & 33.970 & 116.383 & Anthracite & Large & Chen si lou \\
\hline 153 & Henan Province & Shanqiu City & Yong City & 33.941 & 116.376 & Anthracite & Large & Cheng jiao \\
\hline 154 & Henan Province & Shanqiu City & Yong City & 33.920 & 116.627 & Anthracite & Medium & Xin zhuang \\
\hline 155 & Henan Province & Shanqiu City & Yong City & 33.911 & 116.516 & Anthracite & Large & Ju ji \\
\hline
\end{tabular}


Table 1. List of coal mines in China. - Continued

[Abbreviations used: dec. deg. - decimal degrees; NA - not available or not applicable]

\begin{tabular}{|c|c|c|c|c|c|c|c|c|}
\hline $\begin{array}{c}\text { ID } \\
\text { Number }\end{array}$ & $\begin{array}{l}\text { Province, National } \\
\text { Municipality, or } \\
\text { Autonomous Region }\end{array}$ & $\begin{array}{l}\text { Prefecture, Municipality, } \\
\text { Prefecture-Level City, or } \\
\text { League }\end{array}$ & $\begin{array}{l}\text { County, District or } \\
\text { County-Level City }\end{array}$ & $\begin{array}{l}\text { Latitude } \\
\text { (dec. deg.) }\end{array}$ & $\begin{array}{l}\text { Longitude } \\
\text { (dec. deg.) }\end{array}$ & Rank & $\begin{array}{l}\text { Relative } \\
\text { Mine Size }\end{array}$ & Mine Name \\
\hline 156 & Henan Province & Shanqiu City & Yong City & 33.907 & 116.577 & Anthracite & Medium & Ge dian \\
\hline 157 & Henan Province & Shanqiu City & Yong City & 33.845 & 116.306 & Anthracite & Medium & Xin qiao \\
\hline 160 & Henan Province & Zhumadian City & Queshan County & 32.837 & 114.168 & Anthracite & Small & An li \\
\hline 161 & Henan Province & Xinyang City & Gushi County & 31.839 & 115.629 & Anthracite & Small & Yang shan \\
\hline 162 & Henan Province & Xinyang City & Shangcheng County & 31.827 & 115.340 & Anthracite & Small & Ma an shan \\
\hline 18 & Hubei Province & Shiyan City & Zhuxi County & 31.970 & 109.725 & Anthracite & Small & Guang shi ya \\
\hline 19 & Hubei Province & Yichang City & Xingshan County & 31.219 & 110.775 & Bituminous & Small & $\begin{array}{c}\text { Geng jia he Shang bao } \\
\text { Exploration Area }\end{array}$ \\
\hline 20 & Hubei Province & Yichang City & Xingshan County & 31.146 & 110.772 & Bituminous & Small & Zheng jia he \\
\hline 21 & Hubei Province & Yichang City & Zigui County & 31.095 & 110.760 & Bituminous & Small & Yan guan \\
\hline 22 & Hubei Province & $\begin{array}{l}\text { Enshi Autonomous } \\
\text { Prefecture }\end{array}$ & Badong County & 31.053 & 110.449 & Anthracite & Small & Bao ta he \\
\hline 23 & Hubei Province & Xiangyang City & NA & 31.988 & 111.973 & Anthracite & Small & $\begin{array}{l}\text { Long zhong } \\
\quad \text { Ling quan si }\end{array}$ \\
\hline 24 & Hubei Province & Xiangyang City & Nanzhang County & 31.539 & 111.765 & Anthracite & Small & Da ping \\
\hline 25 & Hubei Province & Xiangyang City & Baokang County & 31.502 & 111.438 & Anthracite & Small & Wu hu Chan ge \\
\hline 26 & Hubei Province & Xiangyang City & Baokang County & 31.443 & 111.386 & Bituminous & Small & Gang xi gou \\
\hline 27 & Hubei Province & Xiangyang City & Nanzhang County & 31.310 & 111.839 & Anthracite & Small & Dong gong \\
\hline 28 & Hubei Province & Yichang City & Yuanan County & 31.244 & 111.683 & Anthracite & Small & Tie lu wan \\
\hline 29 & Hubei Province & Yichang City & Yuanan County & 31.232 & 111.809 & Anthracite & Small & Pan long \\
\hline 30 & Hubei Province & Yichang City & NA & 31.205 & 111.491 & Bituminous & Small & $\begin{array}{l}\text { Yua nan, Bai li huang, } \\
\text { Xie jiae } \\
\text { Mining Area }\end{array}$ \\
\hline 31 & Hubei Province & Jingmen City & NA & 31.134 & 111.914 & Anthracite & Small & Ma he Well Field \\
\hline 32 & Hubei Province & Yichang City & NA & 31.057 & 111.478 & Bituminous & Small & $\begin{array}{l}\text { Yua nan, Bai li huang, } \\
\text { Liu shue } \\
\text { Mining Area }\end{array}$ \\
\hline 33 & Hubei Province & Yichang City & Dangyang City & 31.048 & 111.932 & Anthracite & Small & Zhang he Well Field \\
\hline 34 & Hubei Province & Yichang City & $\mathrm{NA}$ & 31.029 & 111.491 & Bituminous & Small & $\begin{array}{l}\text { Yua nan, Bai li huang, } \\
\text { Tian ma } \\
\text { Mining Area }\end{array}$ \\
\hline 35 & Hubei Province & Yichang City & Dangyang City & 31.023 & 111.804 & Anthracite & Small & $\begin{array}{l}\text { San lian wan } \\
\text { Exploration Area }\end{array}$ \\
\hline 36 & Hubei Province & Jingmen City & NA & 31.124 & 112.027 & Anthracite & Small & Dai wan Well Field \\
\hline 37 & Hubei Province & Jingmen City & NA & 31.134 & 112.085 & Anthracite & Small & Guo jia ji \\
\hline
\end{tabular}


Table 1. List of coal mines in China.-Continued

[Abbreviations used: dec. deg. - decimal degrees; NA - not available or not applicable]

\begin{tabular}{|c|c|c|c|c|c|c|c|c|}
\hline $\begin{array}{c}\text { ID } \\
\text { Number }\end{array}$ & $\begin{array}{c}\text { Province, National } \\
\text { Municipality, or } \\
\text { Autonomous Region }\end{array}$ & $\begin{array}{c}\text { Prefecture, Municipality, } \\
\text { Prefecture-Level City, or } \\
\text { League }\end{array}$ & $\begin{array}{l}\text { County, District or } \\
\text { County-Level City }\end{array}$ & $\begin{array}{l}\text { Latitude } \\
\text { (dec. deg.) }\end{array}$ & $\begin{array}{l}\text { Longitude } \\
\text { (dec. deg.) }\end{array}$ & Rank & $\begin{array}{l}\text { Relative } \\
\text { Mine Size }\end{array}$ & Mine Name \\
\hline 38 & Hubei Province & Jingmen City & NA & 31.058 & 112.913 & Bituminous & Small & San yuan kou \\
\hline 39 & Hubei Province & $\begin{array}{l}\text { Enshi Autonomous } \\
\text { Prefecture }\end{array}$ & Lichuan City & 30.398 & 108.723 & Bituminous & Small & Shi dong zi \\
\hline 40 & Hubei Province & $\begin{array}{l}\text { Enshi Autonomous } \\
\text { Prefecture }\end{array}$ & Lichuan City & 30.317 & 108.649 & Bituminous & Small & Shi ba Well Field \\
\hline 41 & Hubei Province & $\begin{array}{l}\text { Enshi Autonomous } \\
\text { Prefecture }\end{array}$ & Lichuan City & 30.239 & 108.601 & Bituminous & Small & Ma luo chi Well Field \\
\hline 42 & Hubei Province & $\begin{array}{l}\text { Enshi Autonomous } \\
\text { Prefecture }\end{array}$ & Lichuan City & 30.096 & 108.608 & Bituminous & Small & Da ma shan Well Field \\
\hline 43 & Hubei Province & $\begin{array}{l}\text { Enshi Autonomous } \\
\text { Prefecture }\end{array}$ & Lichuan City & 30.050 & 108.588 & Bituminous & Small & $\begin{array}{r}\text { Zhuan ya cao } \\
\text { Well Field }\end{array}$ \\
\hline 44 & Hubei Province & $\begin{array}{l}\text { Enshi Autonomous } \\
\text { Prefecture }\end{array}$ & Jianshi County & 30.860 & 109.860 & Anthracite & Small & Xiao jia ping \\
\hline 45 & Hubei Province & $\begin{array}{l}\text { Enshi Autonomous } \\
\text { Prefecture }\end{array}$ & Jianshi County & 30.820 & 109.881 & Anthracite & Small & Mao tian \\
\hline 46 & Hubei Province & $\begin{array}{l}\text { Enshi Autonomous } \\
\text { Prefecture }\end{array}$ & Jianshi County & 30.802 & 109.781 & Anthracite & Small & Tian e chi \\
\hline 47 & Hubei Province & $\begin{array}{l}\text { Enshi Autonomous } \\
\text { Prefecture }\end{array}$ & Jianshi County & 30.798 & 109.963 & Anthracite & Small & $\mathrm{Zi} \mathrm{mu}$ \\
\hline 48 & Hubei Province & $\begin{array}{l}\text { Enshi Autonomous } \\
\text { Prefecture }\end{array}$ & Jianshi County & 30.791 & 109.991 & Anthracite & Small & Liu lin \\
\hline 49 & Hubei Province & $\begin{array}{l}\text { Enshi Autonomous } \\
\text { Prefecture }\end{array}$ & Jianshi County & 30.716 & 109.726 & Anthracite & Small & Huang chang ping \\
\hline 50 & Hubei Province & $\begin{array}{l}\text { Enshi Autonomous } \\
\text { Prefecture }\end{array}$ & NA & 30.642 & 109.575 & Anthracite & Small & Tai yang he \\
\hline 51 & Hubei Province & $\begin{array}{l}\text { Enshi Autonomous } \\
\text { Prefecture }\end{array}$ & NA & 30.523 & 109.610 & Anthracite & Small & Gan ba gou \\
\hline 52 & Hubei Province & $\begin{array}{l}\text { Enshi Autonomous } \\
\text { Prefecture }\end{array}$ & NA & 30.403 & 109.528 & Anthracite & Small & Dong feng \\
\hline 53 & Hubei Province & $\begin{array}{l}\text { Enshi Autonomous } \\
\text { Prefecture }\end{array}$ & NA & 30.345 & 109.526 & Anthracite & Small & Bai jia dong \\
\hline 54 & Hubei Province & $\begin{array}{l}\text { Enshi Autonomous } \\
\text { Prefecture }\end{array}$ & Badong County & 31.003 & 110.214 & Anthracite & Small & Xiao xi he \\
\hline 55 & Hubei Province & Yichang City & Zigui County & 31.000 & 110.746 & Bituminous & Small & $\begin{array}{l}\text { Xiang jia dian } \\
\text { Exploration Area }\end{array}$ \\
\hline 56 & Hubei Province & $\begin{array}{l}\text { Enshi Autonomous } \\
\text { Prefecture }\end{array}$ & Badong County & 30.989 & 110.312 & Anthracite & Small & Wan fu he \\
\hline 57 & Hubei Province & Yichang City & Zigui County & 30.969 & 110.577 & Anthracite & Small & Bai sha \\
\hline 58 & Hubei Province & Yichang City & Zigui County & 30.921 & 110.720 & Bituminous & Small & Guo jia ba \\
\hline
\end{tabular}


Table 1. List of coal mines in China.-Continued

[Abbreviations used: dec. deg. - decimal degrees; NA - not available or not applicable]

\begin{tabular}{|c|c|c|c|c|c|c|c|c|}
\hline $\begin{array}{c}\text { ID } \\
\text { Number }\end{array}$ & $\begin{array}{l}\text { Province, National } \\
\text { Municipality, or } \\
\text { Autonomous Region }\end{array}$ & $\begin{array}{l}\text { Prefecture, Municipality, } \\
\text { Prefecture-Level City, or } \\
\text { League }\end{array}$ & $\begin{array}{l}\text { County, District or } \\
\text { County-Level City }\end{array}$ & $\begin{array}{l}\text { Latitude } \\
\text { (dec. deg.) }\end{array}$ & $\begin{array}{l}\text { Longitude } \\
\text { (dec. deg.) }\end{array}$ & Rank & $\begin{array}{l}\text { Relative } \\
\text { Mine Size }\end{array}$ & Mine Name \\
\hline 59 & Hubei Province & $\begin{array}{l}\text { Enshi Autonomous } \\
\text { Prefecture }\end{array}$ & Badong County & 30.864 & 110.186 & Anthracite & Small & Xin jia \\
\hline 60 & Hubei Province & $\begin{array}{l}\text { Enshi Autonomous } \\
\text { Prefecture }\end{array}$ & Badong County & 30.851 & 110.270 & Anthracite & Small & Lu cong po \\
\hline 61 & Hubei Province & Yichang City & $\begin{array}{l}\text { Changyang } \\
\text { Autonomous County }\end{array}$ & 30.711 & 110.411 & Anthracite & Small & $\begin{array}{l}\text { Ba dong, Ma sha } \\
\text { Well Field }\end{array}$ \\
\hline 62 & Hubei Province & Yichang City & $\begin{array}{l}\text { Changyang } \\
\text { Autonomous County }\end{array}$ & 30.697 & 110.445 & Anthracite & Small & $\begin{array}{l}\text { Ba dong, Zhao gue } \\
\text { Well Field }\end{array}$ \\
\hline 63 & Hubei Province & Yichang City & $\begin{array}{l}\text { Changyang } \\
\text { Autonomous County }\end{array}$ & 30.686 & 110.434 & Anthracite & Small & $\begin{array}{l}\text { Ba dong, Bai guo ping } \\
\text { Well Field }\end{array}$ \\
\hline 64 & Hubei Province & Yichang City & $\begin{array}{l}\text { Changyang } \\
\text { Autonomous County }\end{array}$ & 30.525 & 110.672 & Bituminous & Small & Xiao fen ge \\
\hline 65 & Hubei Province & Yichang City & $\begin{array}{l}\text { Changyang } \\
\text { Autonomous County }\end{array}$ & 30.481 & 110.837 & Bituminous & Small & $\begin{array}{l}\text { Qing gang ping, } \\
\text { Bei ao Well Field }\end{array}$ \\
\hline 66 & Hubei Province & Yichang City & $\begin{array}{l}\text { Changyang } \\
\text { Autonomous County }\end{array}$ & 30.465 & 110.865 & Bituminous & Small & $\begin{array}{l}\text { Qing gang ping, } \\
\text { Well Field }\end{array}$ \\
\hline 67 & Hubei Province & Yichang City & $\begin{array}{l}\text { Changyang } \\
\text { Autonomous County }\end{array}$ & 30.453 & 110.820 & Bituminous & Small & $\begin{array}{l}\text { Ba shan, Tian jia tun } \\
\text { Well Field }\end{array}$ \\
\hline 68 & Hubei Province & Yichang City & $\begin{array}{l}\text { Changyang } \\
\text { Autonomous County }\end{array}$ & 30.419 & 110.756 & Bituminous & Small & $\begin{array}{l}\text { Ba shan, Feng zi ling } \\
\text { Well Field }\end{array}$ \\
\hline 69 & Hubei Province & Yichang City & $\begin{array}{l}\text { Wufeng Autonomous } \\
\text { County }\end{array}$ & 30.125 & 110.969 & Anthracite & Small & Zhang jia Well Field \\
\hline 70 & Hubei Province & $\begin{array}{l}\text { Enshi Autonomous } \\
\text { Prefecture }\end{array}$ & Hefeng County & 30.035 & 110.230 & Anthracite & Small & $\begin{array}{l}\text { Huang jia ying } \\
\text { Hong lian chi }\end{array}$ \\
\hline 71 & Hubei Province & $\begin{array}{l}\text { Enshi Autonomous } \\
\text { Prefecture }\end{array}$ & Hefeng County & 30.024 & 110.286 & Anthracite & Small & Qing hu \\
\hline 72 & Hubei Province & $\begin{array}{l}\text { Enshi Autonomous } \\
\text { Prefecture }\end{array}$ & Hefeng County & 30.022 & 110.130 & Anthracite & Small & Liu jia \\
\hline 73 & Hubei Province & Yichang City & NA & 30.995 & 111.471 & Bituminous & Small & Zhou jia chong \\
\hline 74 & Hubei Province & Yichang City & NA & 30.991 & 111.532 & Bituminous & Small & $\begin{array}{l}\text { Yua nan, Bai li huang } \\
\text { Mining Area }\end{array}$ \\
\hline 75 & Hubei Province & Yichang City & $\begin{array}{l}\text { Changyang } \\
\text { Autonomous County }\end{array}$ & 30.403 & 111.095 & Bituminous & Small & Luo yan shan \\
\hline 76 & Hubei Province & Yichang City & $\begin{array}{l}\text { Changyang } \\
\text { Autonomous County }\end{array}$ & 30.395 & 111.134 & Bituminous & Small & Qin shui ping \\
\hline 77 & Hubei Province & Yichang City & $\begin{array}{l}\text { Changyang } \\
\text { Autonomous County }\end{array}$ & 30.390 & 111.172 & Bituminous & Small & Shi zi bao Well Field \\
\hline 78 & Hubei Province & Yichang City & $\begin{array}{l}\text { Yidu City, } \\
\text { Zhicheng Town }\end{array}$ & 30.153 & 111.425 & Bituminous & Small & Tan zi kou Well Field \\
\hline
\end{tabular}


Table 1. List of coal mines in China. - Continued

[Abbreviations used: dec. deg. - decimal degrees; NA - not available or not applicable]

\begin{tabular}{|c|c|c|c|c|c|c|c|c|}
\hline $\begin{array}{c}\text { ID } \\
\text { Number }\end{array}$ & $\begin{array}{c}\text { Province, National } \\
\text { Municipality, or } \\
\text { Autonomous Region }\end{array}$ & $\begin{array}{c}\text { Prefecture, Municipality, } \\
\text { Prefecture-Level City, or } \\
\text { League }\end{array}$ & $\begin{array}{l}\text { County, District or } \\
\text { County-Level City }\end{array}$ & $\begin{array}{l}\text { Latitude } \\
\text { (dec. deg.) }\end{array}$ & $\begin{array}{l}\text { Longitude } \\
\text { (dec. deg.) }\end{array}$ & Rank & $\begin{array}{l}\text { Relative } \\
\text { Mine Size }\end{array}$ & Mine Name \\
\hline 79 & Hubei Province & Yichang City & $\begin{array}{l}\text { Yidu City, } \\
\quad \text { Zhicheng Town }\end{array}$ & 30.153 & 111.474 & Bituminous & Small & Ge zi tan \\
\hline 80 & Hubei Province & Yichang City & $\begin{array}{l}\text { Yidu City, } \\
\text { Zhicheng Town }\end{array}$ & 30.146 & 111.399 & Bituminous & Small & Chen jia he \\
\hline 81 & Hubei Province & Yichang City & $\begin{array}{l}\text { Yidu City, } \\
\text { Zhicheng Town }\end{array}$ & 30.130 & 111.478 & Bituminous & Small & Hou zi dong jing kou \\
\hline 82 & Hubei Province & Yichang City & $\begin{array}{l}\text { Yidu City, } \\
\text { Zhicheng Town }\end{array}$ & 30.124 & 111.359 & Bituminous & Small & Jian yan he \\
\hline 83 & Hubei Province & Yichang City & $\begin{array}{l}\text { Yidu City, } \\
\text { Zhicheng Town }\end{array}$ & 30.109 & 111.487 & Bituminous & Small & $\begin{array}{l}\text { Liang he kou } \\
\text { Exploration Area }\end{array}$ \\
\hline 84 & Hubei Province & Jinzhou City & Sonzi City & 30.097 & 111.385 & Bituminous & Small & Zhu jia gou Well Field \\
\hline 85 & Hubei Province & Jinzhou City & Sonzi City & 30.093 & 111.422 & Bituminous & Small & $\begin{array}{l}\text { Long keng zi } \\
\text { Exploration Area } \\
\text { (Long Pit) }\end{array}$ \\
\hline 86 & Hubei Province & Jinzhou City & Sonzi City & 30.087 & 111.465 & Bituminous & Small & Shi jia wan \\
\hline 87 & Hubei Province & Xiaogan City & Hanchuan City & 30.308 & 114.073 & Bituminous & Small & $\begin{array}{c}\text { Chen jia shan } \\
\text { Well Field }\end{array}$ \\
\hline 88 & Hubei Province & Ezhou City & NA & 30.302 & 114.873 & Bituminous & Small & Bi shi du \\
\hline 89 & Hubei Province & Wuhan City & Wuchang District & 30.276 & 114.254 & Anthracite & Small & $\begin{array}{l}\text { Guo jia wan } \\
\text { Well Field }\end{array}$ \\
\hline 90 & Hubei Province & Huangshi City & NA & 30.217 & 114.875 & Anthracite & Small & Song ping \\
\hline 91 & Hubei Province & Huangshi City & Daye City & 30.215 & 114.816 & Anthracite & Small & Jin pen Well Field \\
\hline 92 & Hubei Province & Huangshi City & Daye City & 30.211 & 114.671 & Bituminous & Small & $\begin{array}{l}\text { Niu tou shan } \\
\text { Well Field }\end{array}$ \\
\hline 93 & Hubei Province & Huangshi City & Daye City & 30.205 & 114.840 & Anthracite & Small & $\begin{array}{l}\text { Huan di qiao } \\
\text { Well Field }\end{array}$ \\
\hline 94 & Hubei Province & Huangshi City & Daye City & 30.199 & 114.693 & Bituminous & Small & Zhu shu xia Well Field \\
\hline 95 & Hubei Province & Huangshi City & $\mathrm{NA}$ & 30.195 & 114.884 & Anthracite & Small & Xiu shan Well Field \\
\hline 96 & Hubei Province & Huangshi City & Daye City & 30.194 & 114.962 & Anthracite & Small & Bei xia lu Well Field \\
\hline 97 & Hubei Province & Wuhan City & Wuchang District & 30.191 & 114.380 & Bituminous & Small & Ma an shan \\
\hline 98 & Hubei Province & Huangshi City & Daye City & 30.185 & 114.749 & Anthracite & Small & Tong zi gou \\
\hline 99 & Hubei Province & Huangshi City & Daye City & 30.168 & 114.734 & Bituminous & Small & Sha tian \\
\hline 100 & Hubei Province & Huangshi City & Daye City & 30.170 & 115.052 & Bituminous & Small & Xia lu \\
\hline 101 & Hubei Province & Huangshi City & Daye City & 30.157 & 114.872 & Bituminous & Small & $\begin{array}{l}\text { Shui niu shan } \\
\text { Well Field }\end{array}$ \\
\hline 102 & Hubei Province & Wuhan City & Wuchang District & 30.100 & 114.269 & Bituminous & Small & Xin yao Well Field \\
\hline 103 & Hubei Province & Huangshi City & Daye City & 30.043 & 114.944 & Anthracite & Small & Yin shan gou \\
\hline 104 & Hubei Province & Huangshi City & NA & 30.202 & 115.097 & Anthracite & Small & Yuan cang \\
\hline 105 & Hubei Province & Huangshi City & NA & 30.188 & 115.187 & Bituminous & Small & You jia liao Well Field \\
\hline
\end{tabular}


Table 1. List of coal mines in China.-Continued

[Abbreviations used: dec. deg. - decimal degrees; NA - not available or not applicable]

\begin{tabular}{|c|c|c|c|c|c|c|c|c|}
\hline $\begin{array}{c}\text { ID } \\
\text { Number }\end{array}$ & $\begin{array}{c}\text { Province, National } \\
\text { Municipality, or } \\
\text { Autonomous Region }\end{array}$ & $\begin{array}{c}\text { Prefecture, Municipality, } \\
\text { Prefecture-Level City, or } \\
\text { League }\end{array}$ & $\begin{array}{l}\text { County, District or } \\
\text { County-Level City }\end{array}$ & $\begin{array}{l}\text { Latitude } \\
\text { (dec. deg.) }\end{array}$ & $\begin{array}{l}\text { Longitude } \\
\text { (dec. deg.) }\end{array}$ & Rank & $\begin{array}{l}\text { Relative } \\
\text { Mine Size }\end{array}$ & Mine Name \\
\hline 106 & Hubei Province & Huangshi City & NA & 30.182 & 115.143 & Anthracite & Small & Dao shi fu \\
\hline 107 & Hubei Province & Huangshi City & NA & 30.166 & 114.982 & Anthracite & Small & Hu jia wan \\
\hline 108 & Hubei Province & Huangshi City & NA & 30.164 & 115.089 & Anthracite & Small & Yuan hua \\
\hline 109 & Hubei Province & Huangshi City & Daye City & 30.163 & 115.194 & Anthracite & Small & Da lu kou \\
\hline 110 & Hubei Province & Huangshi City & Yangxin County & 30.126 & 115.192 & Bituminous & Small & Wa zui Well Field \\
\hline 111 & Hubei Province & Huangshi City & Yangxin County & 30.120 & 115.264 & Bituminous & Small & $\begin{array}{l}\text { Xia wei shan } \\
\text { Well Field }\end{array}$ \\
\hline 112 & Hubei Province & Huangshi City & Yangxin County & 30.093 & 115.177 & Bituminous & Small & $\begin{array}{l}\text { Hong jia zui } \\
\text { Well Field }\end{array}$ \\
\hline 113 & Hubei Province & Huangshi City & Yangxin County & 30.083 & 115.230 & Anthracite & Small & $\begin{array}{l}\text { Tan shan wan } \\
\text { Well Field }\end{array}$ \\
\hline 114 & Hubei Province & Huangshi City & Yangxin County & 30.082 & 115.262 & Anthracite & Small & Mao cun Well Field \\
\hline 115 & Hubei Province & Huanggang City & Qichun County & 30.026 & 115.368 & Bituminous & Small & Yin shan \\
\hline 116 & Hubei Province & $\begin{array}{l}\text { Enshi Autonomous } \\
\text { Prefecture }\end{array}$ & Lichuan City & 29.989 & 108.583 & Anthracite & Small & Shi zi lu \\
\hline 117 & Hubei Province & $\begin{array}{l}\text { Enshi Autonomous } \\
\text { Prefecture }\end{array}$ & Lichuan City & 29.928 & 108.680 & Anthracite & Small & Jiang kou yan \\
\hline 118 & Hubei Province & $\begin{array}{l}\text { Enshi Autonomous } \\
\text { Prefecture }\end{array}$ & Xianfeng County & 29.532 & 108.973 & Bituminous & Small & Ping shang Well Field \\
\hline 119 & Hubei Province & $\begin{array}{l}\text { Enshi Autonomous } \\
\text { Prefecture }\end{array}$ & Xianfeng County & 29.497 & 108.919 & Anthracite & Small & Yang dong \\
\hline 120 & Hubei Province & $\begin{array}{l}\text { Enshi Autonomous } \\
\text { Prefecture }\end{array}$ & Xianfeng County & 29.482 & 108.906 & Bituminous & Small & Kua yan Well Field \\
\hline 121 & Hubei Province & $\begin{array}{l}\text { Enshi Autonomous } \\
\text { Prefecture }\end{array}$ & Xuan'en County & 29.878 & 109.297 & Anthracite & Small & Xiao guan \\
\hline 122 & Hubei Province & $\begin{array}{l}\text { Enshi Autonomous } \\
\text { Prefecture }\end{array}$ & Laifeng County & 29.481 & 109.352 & Bituminous & Small & Tian ba Well Field \\
\hline 123 & Hubei Province & $\begin{array}{l}\text { Enshi Autonomous } \\
\text { Prefecture }\end{array}$ & Laifeng County & 29.367 & 109.228 & Bituminous & Small & $\begin{array}{l}\text { Sha ping Yuan bao } \\
\text { miao Well Field }\end{array}$ \\
\hline 124 & Hubei Province & $\begin{array}{l}\text { Enshi Autonomous } \\
\text { Prefecture }\end{array}$ & Laifeng County & 29.341 & 109.248 & Bituminous & Small & $\begin{array}{l}\text { Long zui San he gou } \\
\text { Well Field }\end{array}$ \\
\hline 125 & Hubei Province & Xianning City & Jiayu County & 29.901 & 113.933 & Bituminous & Small & $\begin{array}{l}\text { Pao ma ling } \\
\text { Well Field }\end{array}$ \\
\hline 126 & Hubei Province & Xianning City & Jiayu County & 29.824 & 113.875 & Bituminous & Small & Qing shan \\
\hline 127 & Hubei Province & Xianning City & $\begin{array}{l}\text { Cibi City } \\
\text { (formerly Puqi City) }\end{array}$ & 29.765 & 113.776 & Bituminous & Small & $\begin{array}{l}\text { Shen ming shan } \\
\text { Exploration Area }\end{array}$ \\
\hline 128 & Hubei Province & Xianning City & $\begin{array}{l}\text { Cibi City } \\
\quad \text { (formerly Puqi City) }\end{array}$ & 29.719 & 113.858 & Bituminous & Small & $\mathrm{Ku}$ zhu qiao \\
\hline
\end{tabular}


Table 1. List of coal mines in China.-Continued

[Abbreviations used: dec. deg. - decimal degrees; NA - not available or not applicable]

\begin{tabular}{|c|c|c|c|c|c|c|c|c|}
\hline $\begin{array}{l}\text { ID } \\
\text { Number }\end{array}$ & $\begin{array}{c}\text { Province, National } \\
\text { Municipality, or } \\
\text { Autonomous Region }\end{array}$ & $\begin{array}{c}\text { Prefecture, Municipality, } \\
\text { Prefecture-Level City, or } \\
\text { League }\end{array}$ & $\begin{array}{l}\text { County, District or } \\
\text { County-Level City }\end{array}$ & $\begin{array}{l}\text { Latitude } \\
\text { (dec. deg.) }\end{array}$ & $\begin{array}{l}\text { Longitude } \\
\text { (dec. deg.) }\end{array}$ & Rank & $\begin{array}{l}\text { Relative } \\
\text { Mine Size }\end{array}$ & Mine Name \\
\hline 129 & Hubei Province & Xianning City & $\begin{array}{l}\text { Cibi City } \\
\quad \text { (formerly Puqi City) }\end{array}$ & 29.705 & 113.900 & Bituminous & Small & $\begin{array}{l}\text { Xian ren guan } \\
\text { Da feng jian }\end{array}$ \\
\hline 130 & Hubei Province & Xianning City & $\begin{array}{l}\text { Cibi City } \\
\text { (formerly Puqi City) }\end{array}$ & 29.694 & 113.849 & Bituminous & Small & $\begin{array}{l}\text { Ma tu po No. 1-3 } \\
\text { Well Field }\end{array}$ \\
\hline 131 & Hubei Province & Xianning City & $\begin{array}{l}\text { Cibi City } \\
\text { (formerly Puqi City) }\end{array}$ & 29.691 & 113.732 & Anthracite & Small & $\mathrm{Pu}$ shou shan \\
\hline 132 & Hubei Province & Xianning City & $\begin{array}{l}\text { Cibi City } \\
\text { (formerly Puqi City) }\end{array}$ & 29.651 & 113.916 & Bituminous & Small & $\begin{array}{l}\text { Tao hua ping } \\
\text { Exploration Area }\end{array}$ \\
\hline 133 & Hubei Province & Xianning City & $\begin{array}{l}\text { Cibi City } \\
\text { (formerly Puqi City) }\end{array}$ & 29.608 & 113.810 & Bituminous & Small & $\begin{array}{l}\text { Bai shi miao } \\
\text { Well Field }\end{array}$ \\
\hline 134 & Hubei Province & Xianning City & NA & 29.925 & 114.597 & Bituminous & Small & Da wu jin \\
\hline 135 & Hubei Province & Xianning City & NA & 29.909 & 114.558 & Anthracite & Small & Shuang xi \\
\hline 136 & Hubei Province & Huangshi City & Yangxin County & 29.817 & 114.835 & Bituminous & Small & Wu jia chong \\
\hline 137 & Hubei Province & Xianning City & $\begin{array}{l}\text { Cibi City } \\
\quad \text { (formerly Puqi City) }\end{array}$ & 29.747 & 114.158 & Anthracite & Small & Tan po Well Field \\
\hline 138 & Hubei Province & Xianning City & NA & 29.747 & 114.236 & Anthracite & Small & Gu tian \\
\hline 139 & Hubei Province & Xianning City & $\begin{array}{l}\text { Cibi City } \\
\text { (formerly Puqi City) }\end{array}$ & 29.731 & 114.098 & Anthracite & Small & Ye jia ling Well Field \\
\hline 140 & Hubei Province & Xianning City & $\begin{array}{l}\text { Cibi City } \\
\text { (formerly Puqi City) }\end{array}$ & 29.725 & 114.054 & Anthracite & Small & Shuang qiu Well Field \\
\hline 141 & Hubei Province & Xianning City & $\begin{array}{l}\text { Cibi City } \\
\text { (formerly Puqi City) }\end{array}$ & 29.712 & 114.178 & Anthracite & Small & Xiao jia cun \\
\hline 142 & Hubei Province & Xianning City & $\begin{array}{l}\text { Cibi City } \\
\text { (formerly Puqi City) }\end{array}$ & 29.707 & 114.137 & Anthracite & Small & $\begin{array}{l}\text { Zhang si bian } \\
\text { Well Field }\end{array}$ \\
\hline 143 & Hubei Province & Xianning City & $\begin{array}{l}\text { Cibi City } \\
\text { (formerly Puqi City) }\end{array}$ & 29.701 & 114.101 & Anthracite & Small & $\begin{array}{l}\text { You jia shan } \\
\text { Well Field }\end{array}$ \\
\hline 144 & Hubei Province & Xianning City & $\begin{array}{l}\text { Cibi City } \\
\text { (formerly Puqi City) }\end{array}$ & 29.693 & 114.072 & Anthracite & Small & $\begin{array}{l}\text { Lian tang fan } \\
\text { Exploration Area }\end{array}$ \\
\hline 145 & Hubei Province & Xianning City & Tongshan County & 29.671 & 114.828 & Anthracite & Small & Lao hu \\
\hline 146 & Hubei Province & Xianning City & Chongyang County & 29.645 & 114.208 & Anthracite & Small & $\begin{array}{l}\text { Zou ma ling } \\
\text { Well Field }\end{array}$ \\
\hline 147 & Hubei Province & Huangshi City & Yangxin County & 29.565 & 114.923 & Bituminous & Small & Shi xia \\
\hline 148 & Hubei Province & Xianning City & Tongshan County & 29.558 & 114.870 & Bituminous & Small & Pan shan \\
\hline 149 & Hubei Province & Xianning City & Tongshan County & 29.563 & 114.362 & Bituminous & Small & $\begin{array}{l}\text { Lu keng western Well } \\
\text { Field (Lu Pit) }\end{array}$ \\
\hline 150 & Hubei Province & Xianning City & Tongshan County & 29.542 & 114.652 & Bituminous & Small & Heng shi tan \\
\hline 151 & Hubei Province & Xianning City & Chongyang County & 29.469 & 114.184 & Bituminous & Small & Yu shan \\
\hline 152 & Hubei Province & Xianning City & Tongshan County & 29.450 & 114.446 & Bituminous & Small & Cheng shan \\
\hline
\end{tabular}


Table 1. List of coal mines in China. - Continued

[Abbreviations used: dec. deg. - decimal degrees; NA - not available or not applicable]

\begin{tabular}{|c|c|c|c|c|c|c|c|c|}
\hline $\begin{array}{c}\text { ID } \\
\text { Number }\end{array}$ & $\begin{array}{l}\text { Province, National } \\
\text { Municipality, or } \\
\text { Autonomous Region }\end{array}$ & $\begin{array}{l}\text { Prefecture, Municipality, } \\
\text { Prefecture-Level City, or } \\
\text { League }\end{array}$ & $\begin{array}{l}\text { County, District or } \\
\text { County-Level City }\end{array}$ & $\begin{array}{l}\text { Latitude } \\
\text { (dec. deg.) }\end{array}$ & $\begin{array}{l}\text { Longitude } \\
\text { (dec. deg.) }\end{array}$ & Rank & $\begin{array}{l}\text { Relative } \\
\text { Mine Size }\end{array}$ & Mine Name \\
\hline 153 & Hubei Province & Huanggang City & Wuxue City & 29.971 & 115.388 & Bituminous & Small & Huang jia shan \\
\hline 154 & Hubei Province & Huangshi City & Yangxin County & 29.956 & 115.304 & Bituminous & Small & $\begin{array}{l}\text { Meng jia pu } \\
\text { Well Field }\end{array}$ \\
\hline 155 & Hubei Province & Huangshi City & Yangxin County & 29.939 & 115.367 & Bituminous & Small & $\begin{array}{l}\text { Lao du kou } \\
\text { Exploration Area }\end{array}$ \\
\hline 156 & Hubei Province & Huanggang City & Wuxue City & 29.924 & 115.450 & Bituminous & Small & Qi Mine \\
\hline 157 & Hubei Province & Huanggang City & Wuxue City & 29.903 & 115.433 & Bituminous & Small & Tian jia zhen \\
\hline 158 & Hubei Province & Huangshi City & Yangxin County & 29.575 & 115.110 & Bituminous & Small & Sha zi bei Well Field \\
\hline 1 & Hunan Province & Changde City & Shimen County & 30.030 & 110.754 & Anthracite & Small & Jiang ping \\
\hline 2 & Hunan Province & Xiangxi City & Longshan County & 29.236 & 109.516 & Bituminous & Small & Luo ta \\
\hline 3 & Hunan Province & Zhangjiajie City & Sangzhi County & 29.538 & 109.961 & Bituminous & Small & Cha yuan jie \\
\hline 4 & Hunan Province & Zhangjiajie City & Sangzhi County & 29.500 & 109.932 & Bituminous & Small & Yan wu kou \\
\hline 5 & Hunan Province & Zhangjiajie City & Sangzhi County & 29.441 & 109.902 & Bituminous & Small & Xin jie \\
\hline 6 & Hunan Province & $\begin{array}{l}\text { Xiangxi Autonomous } \\
\text { Prefecture }\end{array}$ & Yongshun County & 29.222 & 109.839 & Bituminous & Small & Long jia zhai \\
\hline 7 & Hunan Province & $\begin{array}{l}\text { Xiangxi Autonomous } \\
\text { Prefecture }\end{array}$ & Yongshun County & 29.346 & 110.010 & Bituminous & Small & Guan ba \\
\hline 8 & Hunan Province & Zhangjiajie City & Sangzhi County & 29.442 & 110.097 & Bituminous & Small & $\begin{array}{l}\text { Wo yun jie da gu } \\
\text { Well Field }\end{array}$ \\
\hline 9 & Hunan Province & Zhangjiajie City & Sangzhi County & 29.522 & 110.196 & Bituminous & Small & $\mathrm{Mu}$ xia \\
\hline 10 & Hunan Province & Zhangjiajie City & NA & 29.189 & 110.142 & Bituminous & Small & $\begin{array}{l}\text { Mao gang Mining } \\
\text { Area, Tan mu xi } \\
\text { North Well Field }\end{array}$ \\
\hline 11 & Hunan Province & Zhangjiajie City & Sangzhi County & 29.393 & 110.317 & Bituminous & Small & Fen shui ling \\
\hline 12 & Hunan Province & Zhangjiajie City & Sangzhi County & 29.516 & 110.601 & Bituminous & Small & Bai shi \\
\hline 13 & Hunan Province & Zhangjiajie City & Cili County & 29.702 & 110.878 & Bituminous & Small & Feng he shan \\
\hline 14 & Hunan Province & Zhangjiajie City & Cili County & 29.481 & 111.048 & Bituminous & Small & Ia shue \\
\hline 15 & Hunan Province & Changde City & Shimen County & 29.467 & 111.276 & Bituminous & Small & Long yang wan \\
\hline 16 & Hunan Province & Changde City & Lixian County & 29.837 & 111.406 & Bituminous & Small & Yang er shan \\
\hline 17 & Hunan Province & Linli City & NA & 29.713 & 111.322 & Bituminous & Small & Shi men Yang jiao e \\
\hline 18 & Hunan Province & Linli City & NA & 29.535 & 111.557 & Bituminous & Small & Jiu long qiao \\
\hline 19 & Hunan Province & Changde City & Lixian County & 29.780 & 111.647 & Bituminous & Small & \\
\hline 23 & Hunan Province & $\begin{array}{l}\text { Xiangxi Autonomous } \\
\text { Prefecture }\end{array}$ & Baojing County & 28.641 & 109.239 & Bituminous & Large & Ka peng \\
\hline 24 & Hunan Province & Huaihua City & Yuanling County & 28.258 & 110.345 & Bituminous & Small & $\begin{array}{l}\text { Yuan ling Long yan } \\
\text { tou Well Field }\end{array}$ \\
\hline
\end{tabular}


Table 1. List of coal mines in China.-Continued

[Abbreviations used: dec. deg. - decimal degrees; NA - not available or not applicable]

\begin{tabular}{|c|c|c|c|c|c|c|c|c|}
\hline $\begin{array}{c}\text { ID } \\
\text { Number }\end{array}$ & $\begin{array}{c}\text { Province, National } \\
\text { Municipality, or } \\
\text { Autonomous Region }\end{array}$ & $\begin{array}{c}\text { Prefecture, Municipality, } \\
\text { Prefecture-Level City, or } \\
\text { League }\end{array}$ & $\begin{array}{l}\text { County, District or } \\
\text { County-Level City }\end{array}$ & $\begin{array}{l}\text { Latitude } \\
\text { (dec. deg.) }\end{array}$ & $\begin{array}{l}\text { Longitude } \\
\text { (dec. deg.) }\end{array}$ & Rank & $\begin{array}{l}\text { Relative } \\
\text { Mine Size }\end{array}$ & Mine Name \\
\hline 25 & Hunan Province & Huaihua City & Chenxi County & 28.093 & 110.220 & Bituminous & Small & Chen xi \\
\hline 26 & Hunan Province & Huaihua City & Xupu County & 28.047 & 110.544 & Bituminous & Small & Ma yang shui \\
\hline 27 & Hunan Province & Loudi City & Xinhua County & 28.006 & 111.260 & Anthracite & Small & You xi \\
\hline 28 & Hunan Province & Yiyang City & Anhua County & 27.996 & 111.712 & Bituminous & Small & $\begin{array}{l}\text { Qing tang Hong jia } \\
\text { chong Well Field }\end{array}$ \\
\hline 29 & Hunan Province & Yiyang City & Anhua County & 28.032 & 111.769 & Anthracite & Small & Qing tang \\
\hline 32 & Hunan Province & Yiyang City & Taojiang County & 28.281 & 112.209 & Bituminous & Small & Hui shan gang \\
\hline 33 & Hunan Province & Yiyang City & Taojiang County & 28.279 & 112.305 & Bituminous & Small & $\begin{array}{l}\text { Ba san } 83 \text { Lian he } \\
\text { chong Well Field }\end{array}$ \\
\hline 34 & Hunan Province & Changsha City & Ningxiang County & 28.239 & 112.366 & Bituminous & Small & Mei $\tan$ ba \\
\hline 35 & Hunan Province & Changsha City & Ningxiang County & 28.202 & 112.325 & Bituminous & Small & $\begin{array}{l}\text { Mei tan ba Wu mu } \\
\text { chong Well Field }\end{array}$ \\
\hline 38 & Hunan Province & Changsha City & Liuyang City & 28.322 & 113.759 & Bituminous & Large & Guan du \\
\hline 39 & Hunan Province & Changsha City & NA & 28.044 & 113.047 & Bituminous & Small & Tiao ma dong \\
\hline 40 & Hunan Province & Changsha City & Liuyang City & 28.053 & 113.931 & Anthracite & Small & $\begin{array}{l}\text { Wen jia Zhang jia } \\
\text { chong Well Field }\end{array}$ \\
\hline 41 & Hunan Province & Changsha City & Liuyang City & 28.011 & 113.854 & Bituminous & Small & $\begin{array}{l}\text { Wen jia Da ping } \\
\text { Well Field }\end{array}$ \\
\hline 42 & Hunan Province & Huaihua City & NA & 27.579 & 109.949 & Bituminous & Small & $\begin{array}{l}\text { Yan chong } 8 \\
\text { Well Field }\end{array}$ \\
\hline 43 & Hunan Province & Huaihua City & $\begin{array}{l}\text { Hongjiang City } \\
\text { (formerly Qianyang) }\end{array}$ & 27.381 & 109.933 & Bituminous & Medium & $\begin{array}{c}\text { Shuang xi 3-8 } \\
\text { Well Field }\end{array}$ \\
\hline 44 & Hunan Province & Huaihua City & $\begin{array}{l}\text { Zhijiang Autonomous } \\
\text { County }\end{array}$ & 27.275 & 109.770 & Bituminous & Small & Tie deng po \\
\hline 45 & Hunan Province & Huaihua City & $\begin{array}{l}\text { Hongjiang City } \\
\text { (formerly Qianyang) }\end{array}$ & 27.236 & 109.860 & Bituminous & Small & $\begin{array}{c}\text { Shuang xi 1-2 } \\
\text { Well Field }\end{array}$ \\
\hline 46 & Hunan Province & Huaihua City & Xupu County & 27.959 & 110.533 & Bituminous & Small & Jiao ban xi \\
\hline 47 & Hunan Province & Huaihua City & Chenxi County & 27.876 & 110.199 & Bituminous & Small & No. 51 Mine Area \\
\hline 48 & Hunan Province & Huaihua City & Xupu County & 27.806 & 110.325 & Anthracite & Small & Da jiang kou \\
\hline 49 & Hunan Province & Huaihua City & NA & 27.665 & 110.022 & Bituminous & Small & $\begin{array}{l}\text { Yang chong 1-7 } \\
\text { Well Field }\end{array}$ \\
\hline 50 & Hunan Province & Huaihua City & NA & 27.600 & 110.100 & Bituminous & Small & $\begin{array}{l}\text { Yang chong } 9 \\
\text { Well Field }\end{array}$ \\
\hline 52 & Hunan Province & Loudi City & Lianyuan City & 27.928 & 111.925 & Anthracite & Large & $\begin{array}{l}\text { Xiang bo zheng } \\
\text { fei yue }\end{array}$ \\
\hline 53 & Hunan Province & Loudi City & Lengshuijiang City & 27.820 & 111.487 & Anthracite & Medium & $\begin{array}{r}\text { Leng shui jiang Yang } \\
\text { jia shan Well Field }\end{array}$ \\
\hline 54 & Hunan Province & Loudi City & Xinhua County & 27.813 & 111.590 & Bituminous & Small & Yan jia pu \\
\hline 55 & Hunan Province & Loudi City & Lianyuan City & 27.818 & 111.781 & Bituminous & Small & Qiao tou he \\
\hline
\end{tabular}


Table 1. List of coal mines in China. - Continued

[Abbreviations used: dec. deg. - decimal degrees; NA - not available or not applicable]

\begin{tabular}{|c|c|c|c|c|c|c|c|c|}
\hline $\begin{array}{c}\text { ID } \\
\text { Number }\end{array}$ & $\begin{array}{c}\text { Province, National } \\
\text { Municipality, or } \\
\text { Autonomous Region }\end{array}$ & $\begin{array}{c}\text { Prefecture, Municipality, } \\
\text { Prefecture-Level City, or } \\
\text { League }\end{array}$ & $\begin{array}{l}\text { County, District or } \\
\text { County-Level City }\end{array}$ & $\begin{array}{l}\text { Latitude } \\
\text { (dec. deg.) }\end{array}$ & $\begin{array}{l}\text { Longitude } \\
\text { (dec. deg.) }\end{array}$ & Rank & $\begin{array}{l}\text { Relative } \\
\text { Mine Size }\end{array}$ & Mine Name \\
\hline 56 & Hunan Province & Loudi City & Xinhua County & 27.785 & 111.231 & Anthracite & Small & Lu mao jiang \\
\hline 57 & Hunan Province & Loudi City & Lengshuijiang City & 27.766 & 111.525 & Anthracite & Medium & $\begin{array}{l}\text { Zha du Li bi } \\
\text { Well Field }\end{array}$ \\
\hline 58 & Hunan Province & Loudi City & Lengshuijiang City & 27.772 & 111.641 & Anthracite & Small & Zha du \\
\hline 59 & Hunan Province & Loudi City & Lengshuijiang City & 27.728 & 111.559 & Anthracite & Medium & $\begin{array}{l}\text { Zha du Li min } \\
\text { Well Field }\end{array}$ \\
\hline 60 & Hunan Province & Loudi City & Xinhua County & 27.681 & 111.328 & Bituminous & Small & Ma an shan \\
\hline 61 & Hunan Province & Loudi City & Xinhua County & 27.638 & 111.305 & Anthracite & Small & Jian xin \\
\hline 62 & Hunan Province & Loudi City & Lengshuijiang City & 27.653 & 111.428 & Anthracite & Small & Leng shui jiang \\
\hline 63 & Hunan Province & Loudi City & Lengshuijiang City & 27.636 & 111.507 & Anthracite & Small & Mao yi \\
\hline 64 & Hunan Province & Loudi City & Lengshuijiang City & 27.598 & 111.558 & Anthracite & Small & Jin zhu shan \\
\hline 65 & Hunan Province & Loudi City & NA & 27.690 & 111.926 & Bituminous & Small & Feng guan shan \\
\hline 66 & Hunan Province & Loudi City & Lianyuan City & 27.652 & 111.779 & Bituminous & Small & Dou li shan \\
\hline 67 & Hunan Province & Loudi City & Lianyuan City & 27.605 & 111.819 & Anthracite & Small & Yang jia tan \\
\hline 68 & Hunan Province & Shaoyang City & Xinshao County & 27.375 & 111.166 & Anthracite & Small & Shi zi lu \\
\hline 69 & Hunan Province & Shaoyang City & Xinshao County & 27.335 & 111.360 & Anthracite & Small & Ma jia long \\
\hline 70 & Hunan Province & Shaoyang City & Shaodong County & 27.321 & 111.876 & Anthracite & Small & Lian qiao \\
\hline 71 & Hunan Province & Shaoyang City & NA & 27.298 & 111.547 & Bituminous & Small & Duan po qiao \\
\hline 72 & Hunan Province & Shaoyang City & Shaodong County & 27.261 & 111.643 & Bituminous & Small & Niu ma si \\
\hline 73 & Hunan Province & Shaoyang City & Longhui County & 27.213 & 111.082 & Anthracite & Small & Tan tou \\
\hline 75 & Hunan Province & Shaoyang City & Shaodong County & 27.221 & 111.877 & Bituminous & Small & Bao he tang \\
\hline 76 & Hunan Province & Shaoyang City & Shaodong County & 27.173 & 111.646 & Bituminous & Small & Liang shi tang \\
\hline 77 & Hunan Province & Shaoyang City & Longhui County & 27.104 & 111.185 & Anthracite & Small & $\mathrm{Gu}$ jiao di \\
\hline 78 & Hunan Province & Shaoyang City & NA & 27.059 & 111.290 & Anthracite & Small & Feng jiang xi \\
\hline 79 & Hunan Province & Changsha City & NA & 27.980 & 112.890 & Bituminous & Small & Xiang tan Ping tang \\
\hline 80 & Hunan Province & Changsha City & Ningxiang County & 27.974 & 112.599 & Bituminous & Small & Qing xi chong \\
\hline 81 & Hunan Province & Xiangtan City & Shaoshan City & 27.880 & 112.564 & Bituminous & Small & Yin tian si \\
\hline 82 & Hunan Province & Xiangtan City & NA & 27.868 & 112.670 & Bituminous & Small & Yun hu qiao \\
\hline 83 & Hunan Province & Loudi City & NA & 27.791 & 112.017 & Bituminous & Small & En kou \\
\hline 84 & Hunan Province & Xiangtan City & NA & 27.765 & 112.794 & Bituminous & Small & $\begin{array}{l}\text { Yang jia qiao Mine } \\
\text { Area, Yang jia qiao } \\
\text { Well Field }\end{array}$ \\
\hline 85 & Hunan Province & Xiangtan City & NA & 27.748 & 112.715 & Bituminous & Small & Yang jia qiao \\
\hline 86 & Hunan Province & Loudi City & Shuangfeng County & 27.591 & 112.012 & Bituminous & Small & Hong shan dian \\
\hline 87 & Hunan Province & Loudi City & Shuangfeng County & 27.588 & 112.090 & Anthracite & Small & $\begin{array}{l}\text { Hong shan dian } \\
\text { Qiu hu Well Field }\end{array}$ \\
\hline 88 & Hunan Province & Xiangtan City & NA & 27.632 & 112.980 & Bituminous & Small & Tan jia shan \\
\hline 89 & Hunan Province & Loudi City & Shuangfeng County & 27.534 & 112.103 & Anthracite & Small & Tai ping si \\
\hline
\end{tabular}


Table 1. List of coal mines in China. - Continued

[Abbreviations used: dec. deg. - decimal degrees; NA - not available or not applicable]

\begin{tabular}{|c|c|c|c|c|c|c|c|c|}
\hline $\begin{array}{c}\text { ID } \\
\text { Number }\end{array}$ & $\begin{array}{l}\text { Province, National } \\
\text { Municipality, or } \\
\text { Autonomous Region }\end{array}$ & $\begin{array}{l}\text { Prefecture, Municipality, } \\
\text { Prefecture-Level City, or } \\
\text { League }\end{array}$ & $\begin{array}{l}\text { County, District or } \\
\text { County-Level City }\end{array}$ & $\begin{array}{l}\text { Latitude } \\
\text { (dec. deg.) }\end{array}$ & $\begin{array}{l}\text { Longitude } \\
\text { (dec. deg.) }\end{array}$ & Rank & $\begin{array}{l}\text { Relative } \\
\text { Mine Size }\end{array}$ & Mine Name \\
\hline 90 & Hunan Province & Loudi City & Shuangfeng County & 27.408 & 112.048 & Anthracite & Small & Zhao yang \\
\hline 91 & Hunan Province & Hengyang City & Hengshan County & 27.306 & 112.895 & Anthracite & Small & Xia liu chong \\
\hline 92 & Hunan Province & Hengyang City & Hengshan County & 27.004 & 112.485 & Bituminous & Small & Shan qiao \\
\hline 93 & Hunan Province & Changsha City & Liuyang City & 27.973 & 113.781 & Anthracite & Small & Deng tan jiang \\
\hline 94 & Hunan Province & Zhuzhou City & Liling County & 27.877 & 113.655 & Bituminous & Small & Nan qiao \\
\hline 95 & Hunan Province & Zhuzhou City & Liling County & 27.860 & 113.688 & Bituminous & Small & $\begin{array}{l}\text { Nan qiao, Tong tang } \\
\text { Well Field }\end{array}$ \\
\hline 96 & Hunan Province & Zhuzhou City & Liling County & 27.701 & 113.651 & Anthracite & Small & Da ping shan \\
\hline 97 & Hunan Province & Zhuzhou City & Liling County & 27.576 & 113.441 & Bituminous & Small & Shi men kou \\
\hline 98 & Hunan Province & Zhuzhou City & NA & 27.484 & 113.074 & Bituminous & Small & Hua shi \\
\hline 99 & Hunan Province & Zhuzhou City & Liling County & 27.467 & 113.368 & Anthracite & Small & Da zhang \\
\hline 100 & Hunan Province & Zhuzhou City & Youxian County & 27.245 & 113.669 & Anthracite & Small & $\begin{array}{l}\text { Huang feng qiao, } \\
\text { Lan cun }\end{array}$ \\
\hline 101 & Hunan Province & Hengyang City & Hengdong County & 27.182 & 113.165 & Anthracite & Small & Yang qiao \\
\hline 102 & Hunan Province & Zhuzhou City & Youxian County & 27.131 & 113.229 & Bituminous & Small & $\begin{array}{l}\text { Heng dong, Tao mu } \\
\text { Mining Area, Tan } \\
\text { shan po Well Field }\end{array}$ \\
\hline 103 & Hunan Province & Zhuzhou City & Youxian County & 27.082 & 113.175 & Anthracite & Small & $\begin{array}{l}\text { Heng dong, Tao mu } \\
\text { Mining Area, Wei } \\
\text { tian Well Field }\end{array}$ \\
\hline 104 & Hunan Province & Zhuzhou City & Chaling County & 27.004 & 113.826 & Anthracite & Small & Jie hua long \\
\hline 105 & Hunan Province & Huaihua City & Huitong County & 26.959 & 109.764 & Bituminous & Small & Ping cun \\
\hline 106 & Hunan Province & Jingzhou City & NA & 26.523 & 109.556 & Bituminous & Small & $\begin{array}{l}\text { Jing zhou Pu kou } 2 \\
\text { Well Field }\end{array}$ \\
\hline 107 & Hunan Province & Jingzhou City & NA & 26.459 & 109.495 & Bituminous & Small & Jing zhou \\
\hline 108 & Hunan Province & Shaoyang City & Dongkou County & 26.994 & 110.691 & Bituminous & Small & Shi xia jiang \\
\hline 109 & Hunan Province & Shaoyang City & Xinning County & 26.916 & 110.958 & Anthracite & Small & Deng jia pu \\
\hline 110 & Hunan Province & Shaoyang City & Wugang City & 26.685 & 110.810 & Anthracite & Small & Shuang jiang \\
\hline 111 & Hunan Province & Shaoyang City & Wugang City & 26.613 & 110.694 & Anthracite & Small & Long jiang \\
\hline 112 & Hunan Province & Shaoyang City & Xinning County & 26.601 & 110.937 & Anthracite & Small & Huang long \\
\hline 113 & Hunan Province & Shaoyang City & Wugang City & 26.555 & 110.866 & Anthracite & Small & Li shan yuan \\
\hline 114 & Hunan Province & Shaoyang City & NA & 26.976 & 111.041 & Anthracite & Small & Chang le \\
\hline 115 & Hunan Province & Shaoyang City & NA & 26.918 & 111.191 & Anthracite & Small & San bi tian \\
\hline 116 & Hunan Province & Hengyang City & Qidong County & 26.898 & 111.758 & Bituminous & Small & Jiang zi chong \\
\hline 117 & Hunan Province & Hengyang City & Qidong County & 26.811 & 111.777 & Bituminous & Small & Shi yan Area Section \\
\hline 118 & Hunan Province & Loudi City & Lengshuijiang City & 26.657 & 111.572 & Bituminous & Small & Feng jia chong \\
\hline 119 & Hunan Province & Loudi City & Lengshuijiang City & 26.636 & 111.642 & Bituminous & Small & Dong feng \\
\hline 120 & Hunan Province & Loudi City & Lengshuijiang City & 26.617 & 111.669 & Bituminous & Small & Huang yang si \\
\hline
\end{tabular}


Table 1. List of coal mines in China. - Continued

[Abbreviations used: dec. deg. - decimal degrees; NA - not available or not applicable]

\begin{tabular}{|c|c|c|c|c|c|c|c|c|}
\hline $\begin{array}{c}\text { ID } \\
\text { Number }\end{array}$ & $\begin{array}{l}\text { Province, National } \\
\text { Municipality, or } \\
\text { Autonomous Region }\end{array}$ & $\begin{array}{l}\text { Prefecture, Municipality, } \\
\text { Prefecture-Level City, or } \\
\text { League }\end{array}$ & $\begin{array}{l}\text { County, District or } \\
\text { County-Level City }\end{array}$ & $\begin{array}{l}\text { Latitude } \\
\text { (dec. deg.) }\end{array}$ & $\begin{array}{l}\text { Longitude } \\
\text { (dec. deg.) }\end{array}$ & Rank & $\begin{array}{l}\text { Relative } \\
\text { Mine Size }\end{array}$ & Mine Name \\
\hline 121 & Hunan Province & Loudi City & Lengshuijiang City & 26.585 & 111.636 & Bituminous & Small & Ma jia qiao \\
\hline 122 & Hunan Province & Loudi City & Lengshuijiang City & 26.553 & 111.718 & Bituminous & Small & Xiang hua ba \\
\hline 123 & Hunan Province & Huaihua City & $\begin{array}{l}\text { Hongjiang City } \\
\quad \text { (formerly Qianyang) }\end{array}$ & 26.494 & 111.760 & Bituminous & Small & Wei xing \\
\hline 124 & Hunan Province & Huaihua City & $\begin{array}{l}\text { Hongjiang City } \\
\quad \text { (formerly Qianyang) }\end{array}$ & 26.448 & 111.875 & Bituminous & Small & Guan yin tan \\
\hline 125 & Hunan Province & Huaihua City & $\begin{array}{l}\text { Hongjiang City } \\
\text { (formerly Qianyang) }\end{array}$ & 26.430 & 111.764 & Bituminous & Small & Dong fang hong \\
\hline 126 & Hunan Province & Huaihua City & $\begin{array}{l}\text { Hongjiang City } \\
\text { (formerly Qianyang) }\end{array}$ & 26.404 & 112.003 & Bituminous & Small & Sha jiang \\
\hline 127 & Hunan Province & Huaihua City & $\begin{array}{l}\text { Hongjiang City } \\
\quad \text { (formerly Qianyang) }\end{array}$ & 26.372 & 111.818 & Bituminous & Small & San kou tang \\
\hline 128 & Hunan Province & Hengyang City & Hengnan County & 26.842 & 112.488 & Anthracite & Small & Zhou shi \\
\hline 129 & Hunan Province & Hengyang City & Qidong County & 26.679 & 112.103 & Bituminous & Small & 71 Mining Area \\
\hline 130 & Hunan Province & Hengyang City & Changning City & 26.617 & 112.487 & Bituminous & Small & $\begin{array}{l}\text { Do ling Mining Area, } \\
\text { Wu li ting } \\
\text { Well Field }\end{array}$ \\
\hline 131 & Hunan Province & Hengyang City & Leiyang City & 26.475 & 112.119 & Anthracite & Small & Ma shui \\
\hline 132 & Hunan Province & Hengyang City & Changning City & 26.553 & 112.481 & Bituminous & Small & Dou ling \\
\hline 133 & Hunan Province & Huaihua City & $\begin{array}{l}\text { Hongjiang City } \\
\quad \text { (formerly Qianyang) }\end{array}$ & 26.383 & 112.350 & Bituminous & Small & Huang ni tang \\
\hline 134 & Hunan Province & Hengyang City & Changning City & 26.441 & 112.511 & Anthracite & Small & Yan hu \\
\hline 135 & Hunan Province & Hengyang City & Changning City & 26.377 & 112.564 & Anthracite & Small & Wu fang \\
\hline 136 & Hunan Province & Hengyang City & Changning City & 26.603 & 112.973 & Bituminous & Small & Wu jia qiao \\
\hline 137 & Hunan Province & Hengyang City & Leiyang City & 26.466 & 112.972 & Anthracite & Small & $\begin{array}{l}\text { Bai sha Mining Area, } \\
\text { Nos. 1-7 Well Field }\end{array}$ \\
\hline 138 & Hunan Province & Hengyang City & Leiyang City & 26.426 & 112.935 & Anthracite & Small & $\begin{array}{l}\text { Bai sha Mining Area, } \\
\text { Nos. 8-14 } \\
\text { Well Field }\end{array}$ \\
\hline 139 & Hunan Province & Hengyang City & Leiyang City & 26.335 & 112.863 & Anthracite & Small & $\begin{array}{l}\text { Bai sha Mining Area, } \\
\text { Nos. 15-19 } \\
\text { Well Field }\end{array}$ \\
\hline 140 & Hunan Province & Hengyang City & Leiyang City & 26.263 & 112.875 & Anthracite & Small & $\begin{array}{l}\text { Bai sha Mining Area, } \\
\text { Nos. } 20-23 \\
\text { Well Field }\end{array}$ \\
\hline 141 & Hunan Province & Chenzhou City & Yongxing County & 26.147 & 112.867 & Anthracite & Small & Ma tian \\
\hline 142 & Hunan Province & Hengyang City & Leiyang City & 26.389 & 113.001 & Anthracite & Small & $\begin{array}{l}\text { Yong xing Yong lai } \\
\text { Mining Area, Nos. } \\
\text { 1-3 Well Field }\end{array}$ \\
\hline
\end{tabular}


Table 1. List of coal mines in China. - Continued

[Abbreviations used: dec. deg. - decimal degrees; NA - not available or not applicable]

\begin{tabular}{|c|c|c|c|c|c|c|c|c|}
\hline $\begin{array}{c}\text { ID } \\
\text { Number }\end{array}$ & $\begin{array}{l}\text { Province, National } \\
\text { Municipality, or } \\
\text { Autonomous Region }\end{array}$ & $\begin{array}{c}\text { Prefecture, Municipality, } \\
\text { Prefecture-Level City, or } \\
\text { League }\end{array}$ & $\begin{array}{l}\text { County, District or } \\
\text { County-Level City }\end{array}$ & $\begin{array}{l}\text { Latitude } \\
\text { (dec. deg.) }\end{array}$ & $\begin{array}{l}\text { Longitude } \\
\text { (dec. deg.) }\end{array}$ & Rank & $\begin{array}{l}\text { Relative } \\
\text { Mine Size }\end{array}$ & Mine Name \\
\hline 143 & Hunan Province & Hengyang City & Leiyang City & 26.279 & 113.000 & Anthracite & Small & $\begin{array}{l}\text { Yong xing Yong lai } \\
\text { Mining Area, Nos. } 4 \\
\text { Well Field }\end{array}$ \\
\hline 144 & Hunan Province & Hengyang City & Leiyang City & 26.211 & 113.030 & Anthracite & Small & $\begin{array}{l}\text { Yong xing Yong lai } \\
\text { Mining Area, Nos. } \\
\text { 9-21 Well Field }\end{array}$ \\
\hline 145 & Hunan Province & Hengyang City & Leiyang City & 26.438 & 113.071 & Anthracite & Small & $\begin{array}{l}\text { Yong xing Yong lai } \\
\text { Mining Area, Nos. } \\
\text { 5-8 Well Field }\end{array}$ \\
\hline 146 & Hunan Province & Hengyang City & Leiyang City & 26.472 & 113.185 & Anthracite & Small & $\begin{array}{l}\text { Yong xing Yong lai } \\
\text { Mining Area, Nos. } \\
\text { 22-24 Well Field }\end{array}$ \\
\hline 147 & Hunan Province & Chenzhou City & Yongxing County & 26.151 & 113.270 & Bituminous & Small & Gao po \\
\hline 148 & Hunan Province & Chenzhou City & Zixing City & 26.065 & 113.285 & Bituminous & Small & $\begin{array}{l}\text { San dou Yu yu Xia } \\
\text { ping dong } \\
\text { Well Field }\end{array}$ \\
\hline 149 & Hunan Province & Chenzhou City & Zixing City & 26.019 & 113.271 & Bituminous & Small & $\begin{array}{l}\text { San dou Zhou yuan } \\
\text { shan Well Field }\end{array}$ \\
\hline 150 & Hunan Province & Zhuzhou City & Yanling County & 26.462 & 113.635 & Bituminous & Small & Huo jia \\
\hline 151 & Hunan Province & Yongzhou City & Jiangyong County & 25.148 & 111.105 & Bituminous & Small & $\begin{array}{l}\text { Tao chuan Mining } \\
\text { Area, No. } 3 \\
\text { Well Field }\end{array}$ \\
\hline 152 & Hunan Province & Yongzhou City & Jiangyong County & 25.121 & 111.049 & Anthracite & Small & Tao chuan \\
\hline 153 & Hunan Province & Chenzhou City & NA & 25.959 & 112.958 & Anthracite & Small & Jie dong \\
\hline 154 & Hunan Province & Chenzhou City & NA & 25.779 & 112.828 & Anthracite & Small & Hua tang \\
\hline 155 & Hunan Province & Chenzhou City & Guiyang County & 25.683 & 112.602 & Anthracite & Small & San wu chong \\
\hline 156 & Hunan Province & Chenzhou City & Jianhe County & 25.600 & 112.450 & Anthracite & Small & Yuan jia \\
\hline 157 & Hunan Province & Chenzhou City & Guiyang County & 25.564 & 112.724 & Anthracite & Small & Lu tang \\
\hline 158 & Hunan Province & Chenzhou City & Yizhang County & 25.367 & 112.820 & Anthracite & Small & Mei tian \\
\hline 159 & Hunan Province & Chenzhou City & Zixing City & 25.980 & 113.255 & Bituminous & Small & San dou \\
\hline 160 & Hunan Province & Chenzhou City & NA & 25.888 & 113.010 & Anthracite & Small & $\mathrm{Xu}$ jia dong \\
\hline 161 & Hunan Province & Chenzhou City & Guidong County & 25.461 & 113.093 & Anthracite & Small & Sha tian \\
\hline 162 & Hunan Province & Chenzhou City & Rucheng County & 25.530 & 113.206 & Anthracite & Small & Liang tian \\
\hline 163 & Hunan Province & Chenzhou City & Yizhang County & 25.808 & 113.781 & Anthracite & Small & Yan mei shan \\
\hline 1 & $\begin{array}{l}\text { Inner Mongolia } \\
\text { Autonomous Region }\end{array}$ & $\begin{array}{l}\text { Hulunbuir City } \\
\text { (Hulunbeier Shi) }\end{array}$ & $\begin{array}{l}\text { Manzhouli } \\
\text { (Sub-Prefecture- } \\
\text { Level) City }\end{array}$ & 49.565 & 117.266 & Lignite & Small & Kai fang shan \\
\hline
\end{tabular}


Table 1. List of coal mines in China.-Continued

[Abbreviations used: dec. deg. - decimal degrees; NA - not available or not applicable]

\begin{tabular}{|c|c|c|c|c|c|c|c|c|}
\hline $\begin{array}{l}\text { ID } \\
\text { Number }\end{array}$ & $\begin{array}{c}\text { Province, National } \\
\text { Municipality, or } \\
\text { Autonomous Region }\end{array}$ & $\begin{array}{c}\text { Prefecture, Municipality, } \\
\text { Prefecture-Level City, or } \\
\text { League }\end{array}$ & $\begin{array}{l}\text { County, District or } \\
\text { County-Level City }\end{array}$ & $\begin{array}{l}\text { Latitude } \\
\text { (dec. deg.) }\end{array}$ & $\begin{array}{l}\text { Longitude } \\
\text { (dec. deg.) }\end{array}$ & Rank & $\begin{array}{l}\text { Relative } \\
\text { Mine Size }\end{array}$ & Mine Name \\
\hline 2 & $\begin{array}{l}\text { Inner Mongolia } \\
\text { Autonomous Region }\end{array}$ & $\begin{array}{l}\text { Hulunbuir City } \\
\text { (Hulunbeier Shi) }\end{array}$ & $\begin{array}{l}\text { Manzhouli } \\
\text { (Sub-Prefecture- } \\
\text { Level) City }\end{array}$ & 49.471 & 117.850 & Lignite & Large & Xi shan \\
\hline 3 & $\begin{array}{l}\text { Inner Mongolia } \\
\text { Autonomous Region }\end{array}$ & $\begin{array}{l}\text { Hulunbuir City } \\
\text { (Hulunbeier Shi) }\end{array}$ & $\begin{array}{l}\text { Manzhouli } \\
\text { (Sub-Prefecture- } \\
\text { Level) City }\end{array}$ & 49.471 & 117.850 & Lignite & Large & Ling quan \\
\hline 4 & $\begin{array}{l}\text { Inner Mongolia } \\
\text { Autonomous Region }\end{array}$ & $\begin{array}{l}\text { Hulunbuir City } \\
\text { (Hulunbeier Shi) }\end{array}$ & $\begin{array}{l}\text { Manzhouli } \\
\text { (Sub-Prefecture- } \\
\text { Level) City }\end{array}$ & 49.471 & 117.850 & Lignite & Large & Ling bei \\
\hline 5 & $\begin{array}{l}\text { Inner Mongolia } \\
\text { Autonomous Region }\end{array}$ & $\begin{array}{l}\text { Hulunbuir City } \\
\text { (Hulunbeier Shi) }\end{array}$ & $\begin{array}{l}\text { Manzhouli } \\
\text { (Sub-Prefecture- } \\
\text { Level) City }\end{array}$ & 49.471 & 117.850 & Lignite & Large & Lu tian Open Pit Mine \\
\hline 6 & $\begin{array}{l}\text { Inner Mongolia } \\
\text { Autonomous Region }\end{array}$ & $\begin{array}{l}\text { Hulunbuir City } \\
\text { (Hulunbeier Shi) }\end{array}$ & $\begin{array}{l}\text { Manzhouli } \\
\text { (Sub-Prefecture- } \\
\text { Level) City }\end{array}$ & 49.376 & 117.759 & Lignite & Large & Tie bei \\
\hline 7 & $\begin{array}{l}\text { Inner Mongolia } \\
\text { Autonomous Region }\end{array}$ & $\begin{array}{l}\text { Hulunbuir City } \\
\text { (Hulunbeier Shi) }\end{array}$ & $\begin{array}{l}\text { Manzhouli } \\
\text { (Sub-Prefecture- } \\
\text { Level) City }\end{array}$ & 49.263 & 117.446 & Lignite & Large & $\begin{array}{l}\text { Ling dong } \\
\text { Reconnaissance } \\
\text { Area }\end{array}$ \\
\hline 8 & $\begin{array}{l}\text { Inner Mongolia } \\
\text { Autonomous Region }\end{array}$ & $\begin{array}{l}\text { Hulunbuir City } \\
\text { (Hulunbeier Shi) }\end{array}$ & $\begin{array}{l}\text { Old Barag Banner } \\
\text { (Chenbaerhu Qi) }\end{array}$ & 49.361 & 119.596 & Lignite & Large & Bao ri xi le \\
\hline 9 & $\begin{array}{l}\text { Inner Mongolia } \\
\text { Autonomous Region }\end{array}$ & $\begin{array}{l}\text { Hulunbuir City } \\
\text { (Hulunbeier Shi) }\end{array}$ & $\begin{array}{l}\text { Evenk Autonomous } \\
\text { Banner } \\
\text { (Ewenkezu Ziahiqi) }\end{array}$ & 48.942 & 119.661 & Lignite & Medium & $\begin{array}{l}\text { Ma da mu ji pen di } \\
\text { Don duan zhao } \\
\text { mei qu }\end{array}$ \\
\hline 10 & $\begin{array}{l}\text { Inner Mongolia } \\
\text { Autonomous Region }\end{array}$ & $\begin{array}{l}\text { Hulunbuir City } \\
\text { (Hulunbeier Shi) }\end{array}$ & $\begin{array}{l}\text { Evenk Autonomous } \\
\text { Banner } \\
\text { (Ewenkezu Ziahiqi) }\end{array}$ & 48.684 & 119.639 & Lignite & Large & Yi min \\
\hline 11 & $\begin{array}{l}\text { Inner Mongolia } \\
\text { Autonomous Region }\end{array}$ & $\begin{array}{l}\text { Hulunbuir City } \\
\text { (Hulunbeier Shi) }\end{array}$ & $\begin{array}{l}\text { Ergun City (formerly } \\
\text { Ergun Right Banner } \\
\text { (Eergunayou Qi)) }\end{array}$ & 50.287 & 120.226 & Bituminous & Small & La bu da lin \\
\hline 12 & $\begin{array}{l}\text { Inner Mongolia } \\
\text { Autonomous Region }\end{array}$ & $\begin{array}{l}\text { Hulunbuir City } \\
\text { (Hulunbeier Shi) }\end{array}$ & Yakeshi City & 49.552 & 121.521 & Bituminous & Small & Wu jiu \\
\hline 13 & $\begin{array}{l}\text { Inner Mongolia } \\
\text { Autonomous Region }\end{array}$ & $\begin{array}{l}\text { Hulunbuir City } \\
\text { (Hulunbeier Shi) }\end{array}$ & $\begin{array}{l}\text { Evenk Autonomous } \\
\text { Banner } \\
\text { (Ewenkezu Ziahiqi) }\end{array}$ & 49.243 & 120.643 & Lignite & Medium & Da yan \\
\hline 14 & $\begin{array}{l}\text { Inner Mongolia } \\
\text { Autonomous Region }\end{array}$ & $\begin{array}{l}\text { Hulunbuir City } \\
\text { (Hulunbeier Shi) }\end{array}$ & Yakeshi City & 49.075 & 120.888 & Bituminous & Small & Mian du he \\
\hline 15 & $\begin{array}{l}\text { Inner Mongolia } \\
\text { Autonomous Region }\end{array}$ & $\begin{array}{l}\text { Hulunbuir City } \\
\text { (Hulunbeier Shi) }\end{array}$ & $\begin{array}{l}\text { Oroqin Autonomous } \\
\text { Banner } \\
\text { (Elunchunzu Ziahiqi) }\end{array}$ & 49.772 & 124.761 & Bituminous & Small & Da er bin No. 1 Area \\
\hline
\end{tabular}


Table 1. List of coal mines in China.-Continued

[Abbreviations used: dec. deg. - decimal degrees; NA - not available or not applicable]

\begin{tabular}{|c|c|c|c|c|c|c|c|c|}
\hline $\begin{array}{c}\text { ID } \\
\text { Number }\end{array}$ & $\begin{array}{l}\text { Province, National } \\
\text { Municipality, or } \\
\text { Autonomous Region }\end{array}$ & $\begin{array}{c}\text { Prefecture, Municipality, } \\
\text { Prefecture-Level City, or } \\
\text { League }\end{array}$ & $\begin{array}{l}\text { County, District or } \\
\text { County-Level City }\end{array}$ & $\begin{array}{l}\text { Latitude } \\
\text { (dec. deg.) }\end{array}$ & $\begin{array}{l}\text { Longitude } \\
\text { (dec. deg.) }\end{array}$ & Rank & $\begin{array}{l}\text { Relative } \\
\text { Mine Size }\end{array}$ & Mine Name \\
\hline 16 & $\begin{array}{l}\text { Inner Mongolia } \\
\text { Autonomous Region }\end{array}$ & $\begin{array}{l}\text { Hulunbuir City } \\
\text { (Hulunbeier Shi) }\end{array}$ & $\begin{array}{l}\text { Oroqin Autonomous } \\
\text { Banner } \\
\text { (Elunchunzu Ziahiqi) }\end{array}$ & 49.733 & 124.594 & Bituminous & Small & Da yang shu \\
\hline 17 & $\begin{array}{l}\text { Inner Mongolia } \\
\text { Autonomous Region }\end{array}$ & $\begin{array}{l}\text { Xilingol League } \\
\text { (Xilinguole Meng) }\end{array}$ & $\begin{array}{l}\text { East Ujimqin Banner } \\
\text { (Dongwuzhumuqin } \\
\text { Qi) }\end{array}$ & 45.268 & 115.757 & Bituminous & Small & Ha ma miao \\
\hline 18 & $\begin{array}{l}\text { Inner Mongolia } \\
\text { Autonomous Region }\end{array}$ & $\begin{array}{l}\text { Xilingol League } \\
\text { (Xilinguole Meng) }\end{array}$ & $\begin{array}{l}\text { East Ujimqin Banner } \\
\text { (Dongwuzhumuqin } \\
\text { Qi) }\end{array}$ & 45.188 & 115.472 & Bituminous & Small & Qu li he hu du ga \\
\hline 19 & $\begin{array}{l}\text { Inner Mongolia } \\
\text { Autonomous Region }\end{array}$ & $\begin{array}{l}\text { Xilingol League } \\
\text { (Xilinguole Meng) }\end{array}$ & $\begin{array}{l}\text { East Ujimqin Banner } \\
\text { (Dongwuzhumuqin } \\
\text { Qi) }\end{array}$ & 45.124 & 115.758 & Bituminous & Small & Xin miao \\
\hline 20 & $\begin{array}{l}\text { Inner Mongolia } \\
\text { Autonomous Region }\end{array}$ & $\begin{array}{l}\text { Xilingol League } \\
\text { (Xilinguole Meng) }\end{array}$ & $\begin{array}{l}\text { East Ujimqin Banner } \\
\text { (Dongwuzhumuqin } \\
\text { Qi) }\end{array}$ & 45.058 & 115.419 & Bituminous & Small & Ma ni te miao \\
\hline 21 & $\begin{array}{l}\text { Inner Mongolia } \\
\text { Autonomous Region }\end{array}$ & $\begin{array}{l}\text { Xilingol League } \\
\text { (Xilinguole Meng) }\end{array}$ & $\begin{array}{l}\text { Abag Banner } \\
\text { (Abaga Qi) }\end{array}$ & 44.634 & 115.292 & Bituminous & Small & Bai yin hu du ga \\
\hline 22 & $\begin{array}{l}\text { Inner Mongolia } \\
\text { Autonomous Region }\end{array}$ & $\begin{array}{l}\text { Xilingol League } \\
\text { (Xilinguole Meng) }\end{array}$ & $\begin{array}{l}\text { Sonid Left Banner } \\
\text { (Sunite Zuo Qi) }\end{array}$ & 44.048 & 113.298 & Lignite & Medium & $\begin{array}{l}\text { Bai yin wu la Coal } \\
\text { Exploration Area }\end{array}$ \\
\hline 23 & $\begin{array}{l}\text { Inner Mongolia } \\
\text { Autonomous Region }\end{array}$ & $\begin{array}{l}\text { Xilingol League } \\
\quad \text { (Xilinguole Meng) }\end{array}$ & $\begin{array}{l}\text { East Ujimqin Banner } \\
\text { (Dongwuzhumuqin } \\
\text { Qi) }\end{array}$ & 45.898 & 119.675 & Lignite & Small & Wu qi jun ma chang \\
\hline 24 & $\begin{array}{l}\text { Inner Mongolia } \\
\text { Autonomous Region }\end{array}$ & $\begin{array}{l}\text { Tongliao City } \\
\text { (Tongliao Shi) }\end{array}$ & $\begin{array}{l}\text { Holingol City } \\
\text { (Huolinguole Shi) }\end{array}$ & 45.469 & 119.532 & Lignite & Large & He lin he \\
\hline 25 & $\begin{array}{l}\text { Inner Mongolia } \\
\text { Autonomous Region }\end{array}$ & $\begin{array}{l}\text { Xilingol League } \\
\text { (Xilinguole Meng) }\end{array}$ & $\begin{array}{l}\text { West Ujimqin Banner } \\
\text { (Xiwuzhumuqin Qi) }\end{array}$ & 45.036 & 118.663 & Lignite & Large & $\begin{array}{l}\text { Bai yin hua } \\
\text { Exploration Area }\end{array}$ \\
\hline 26 & $\begin{array}{l}\text { Inner Mongolia } \\
\quad \text { Autonomous Region }\end{array}$ & $\begin{array}{l}\text { Xilingol League } \\
\text { (Xilinguole Meng) }\end{array}$ & $\begin{array}{l}\text { West Ujimqin Banner } \\
\text { (Xiwuzhumuqin Qi) }\end{array}$ & 44.779 & 117.647 & Lignite & Medium & $\begin{array}{c}\text { Ba yan hu shuo Coal } \\
\text { Exploration Area }\end{array}$ \\
\hline 27 & $\begin{array}{l}\text { Inner Mongolia } \\
\text { Autonomous Region }\end{array}$ & $\begin{array}{l}\text { Xilingol League } \\
\text { (Xilinguole Meng) }\end{array}$ & $\begin{array}{l}\text { West Ujimqin Banner } \\
\text { (Xiwuzhumuqin Qi) }\end{array}$ & 44.569 & 117.468 & Bituminous & Small & Jiao er qi ge \\
\hline 28 & $\begin{array}{l}\text { Inner Mongolia } \\
\text { Autonomous Region }\end{array}$ & $\begin{array}{l}\text { Xilingol League } \\
\text { (Xilinguole Meng) }\end{array}$ & $\begin{array}{l}\text { West Ujimqin Banner } \\
\text { (Xiwuzhumuqin Qi) }\end{array}$ & 44.507 & 117.612 & Bituminous & Small & Huo zi liang \\
\hline 29 & $\begin{array}{l}\text { Inner Mongolia } \\
\text { Autonomous Region }\end{array}$ & $\begin{array}{l}\text { Xilingol League } \\
\text { (Xilinguole Meng) }\end{array}$ & $\begin{array}{l}\text { West Ujimqin Banner } \\
\text { (Xiwuzhumuqin Qi) }\end{array}$ & 44.546 & 117.751 & Bituminous & Small & Exploration Area \\
\hline 30 & $\begin{array}{l}\text { Inner Mongolia } \\
\text { Autonomous Region }\end{array}$ & $\begin{array}{l}\text { Xilingol League } \\
\text { (Xilinguole Meng) }\end{array}$ & $\begin{array}{l}\text { West Ujimqin Banner } \\
\text { (Xiwuzhumuqin Qi) }\end{array}$ & 44.288 & 117.348 & Lignite & Medium & Ji lin guo le \\
\hline 31 & $\begin{array}{l}\text { Inner Mongolia } \\
\text { Autonomous Region }\end{array}$ & $\begin{array}{l}\text { Xilingol League } \\
\text { (Xilinguole Meng) }\end{array}$ & $\begin{array}{l}\text { Xilinhot City } \\
\text { (Xilinhaote Shi) }\end{array}$ & 44.207 & 116.306 & Lignite & Medium & $\begin{array}{l}\text { Ba yan bao li ge Coal } \\
\text { Exploration Area }\end{array}$ \\
\hline
\end{tabular}


Table 1. List of coal mines in China.-Continued

[Abbreviations used: dec. deg. - decimal degrees; NA - not available or not applicable]

\begin{tabular}{|c|c|c|c|c|c|c|c|c|}
\hline $\begin{array}{l}\text { ID } \\
\text { Number }\end{array}$ & $\begin{array}{c}\text { Province, National } \\
\text { Municipality, or } \\
\text { Autonomous Region }\end{array}$ & $\begin{array}{c}\text { Prefecture, Municipality, } \\
\text { Prefecture-Level City, or } \\
\text { League }\end{array}$ & $\begin{array}{l}\text { County, District or } \\
\text { County-Level City }\end{array}$ & $\begin{array}{l}\text { Latitude } \\
\text { (dec. deg.) }\end{array}$ & $\begin{array}{l}\text { Longitude } \\
\text { (dec. deg.) }\end{array}$ & Rank & $\begin{array}{l}\text { Relative } \\
\text { Mine Size }\end{array}$ & Mine Name \\
\hline 32 & $\begin{array}{l}\text { Inner Mongolia } \\
\text { Autonomous Region }\end{array}$ & $\begin{array}{l}\text { Xilingol League } \\
\text { (Xilinguole Meng) }\end{array}$ & $\begin{array}{l}\text { Xilinhot City } \\
\text { (Xilinhaote Shi) }\end{array}$ & 44.050 & 116.054 & Lignite & Large & Sheng li Coal Field \\
\hline 33 & $\begin{array}{l}\text { Inner Mongolia } \\
\text { Autonomous Region }\end{array}$ & $\begin{array}{l}\text { Xilingol League } \\
\text { (Xilinguole Meng) }\end{array}$ & $\begin{array}{l}\text { Xilinhot City } \\
\text { (Xilinhaote Shi) }\end{array}$ & 44.050 & 116.054 & Lignite & Large & $\begin{array}{l}\text { Hong qi } \\
\text { Open Pit Mine }\end{array}$ \\
\hline 34 & $\begin{array}{l}\text { Inner Mongolia } \\
\text { Autonomous Region }\end{array}$ & $\begin{array}{l}\text { Xilingol League } \\
\text { (Xilinguole Meng) }\end{array}$ & $\begin{array}{l}\text { Xilinhot City } \\
\text { (Xilinhaote Shi) }\end{array}$ & 43.977 & 116.169 & Bituminous & Small & $\begin{array}{l}\text { Chu gu lan } \\
\text { "Mining Area }\end{array}$ \\
\hline 35 & $\begin{array}{l}\text { Inner Mongolia } \\
\text { Autonomous Region }\end{array}$ & $\begin{array}{l}\text { Xilingol League } \\
\quad \text { (Xilinguole Meng) }\end{array}$ & $\begin{array}{l}\text { Xilinhot City } \\
\text { (Xilinhaote Shi) }\end{array}$ & 43.977 & 116.169 & Bituminous & Small & $\mathrm{Xi}$ lin hao te \\
\hline 36 & $\begin{array}{l}\text { Inner Mongolia } \\
\text { Autonomous Region }\end{array}$ & $\begin{array}{l}\text { Hinggan League } \\
\text { (Xing'an Meng) }\end{array}$ & $\begin{array}{l}\text { Tuquan County } \\
\text { (Tuquan Xian) }\end{array}$ & 45.669 & 121.746 & Anthracite & Small & Yu min \\
\hline 37 & $\begin{array}{l}\text { Inner Mongolia } \\
\quad \text { Autonomous Region }\end{array}$ & $\begin{array}{l}\text { Hinggan League } \\
\text { (Xing'an Meng) }\end{array}$ & $\begin{array}{l}\text { Tuquan County } \\
\text { (Tuquan Xian) }\end{array}$ & 45.222 & 121.812 & Bituminous & Small & Niu hai \\
\hline 38 & $\begin{array}{l}\text { Inner Mongolia } \\
\text { Autonomous Region }\end{array}$ & $\begin{array}{l}\text { Hinggan League } \\
\text { (Xing'an Meng) }\end{array}$ & $\begin{array}{l}\text { Jalaid Banner } \\
\text { (Zalaite Qi) }\end{array}$ & 44.722 & 120.523 & Anthracite & Small & Ju li hei \\
\hline 39 & $\begin{array}{l}\text { Inner Mongolia } \\
\text { Autonomous Region }\end{array}$ & $\begin{array}{l}\text { Hinggan League } \\
\text { (Xing'an Meng) }\end{array}$ & $\begin{array}{l}\text { Jalaid Banner } \\
\text { (Zalaite Qi) }\end{array}$ & 44.555 & 120.551 & Anthracite & Small & Gong ying zi \\
\hline 40 & $\begin{array}{l}\text { Inner Mongolia } \\
\text { Autonomous Region }\end{array}$ & $\begin{array}{l}\text { Hinggan League } \\
\text { (Xing'an Meng) }\end{array}$ & $\begin{array}{l}\text { Jalaid Banner } \\
\quad \text { (Zalaite Qi) }\end{array}$ & 44.666 & 121.343 & Bituminous & Small & Wue ge qi \\
\hline 41 & $\begin{array}{l}\text { Inner Mongolia } \\
\text { Autonomous Region }\end{array}$ & $\begin{array}{l}\text { Hinggan League } \\
\text { (Xing'an Meng) }\end{array}$ & $\begin{array}{l}\text { Jalaid Banner } \\
\quad \text { (Zalaite Qi) }\end{array}$ & 44.621 & 121.212 & Anthracite & Small & Huang hua shan \\
\hline 42 & $\begin{array}{l}\text { Inner Mongolia } \\
\text { Autonomous Region }\end{array}$ & $\begin{array}{l}\text { Alxa League } \\
\quad \text { (Alashan Meng) }\end{array}$ & Ejin Banner (Ejina Qi) & 42.353 & 100.056 & Bituminous & Small & $\begin{array}{l}\text { Xi re ha da } \\
\text { Exploration Area }\end{array}$ \\
\hline 43 & $\begin{array}{l}\text { Inner Mongolia } \\
\text { Autonomous Region }\end{array}$ & $\begin{array}{l}\text { Alxa League } \\
\text { (Alashan Meng) }\end{array}$ & Ejin Banner (Ejina Qi) & 41.050 & 98.505 & Anthracite & Small & Sha po quan \\
\hline 44 & $\begin{array}{l}\text { Inner Mongolia } \\
\text { Autonomous Region }\end{array}$ & Baotou City (Baotou Shi) & $\begin{array}{l}\text { Darhan Muminggan } \\
\text { United Banner } \\
\text { (Da'erhan } \\
\text { Maoming'an Lianhe } \\
\text { Qi) }\end{array}$ & 42.322 & 109.710 & Lignite & Large & Bai yan hua \\
\hline 45 & $\begin{array}{l}\text { Inner Mongolia } \\
\text { Autonomous Region }\end{array}$ & $\begin{array}{l}\text { Bayannur City } \\
\text { (Bayannao'er Shi) }\end{array}$ & $\begin{array}{l}\text { Urat Middle Banner } \\
\text { (Wulate Zhong Qi) }\end{array}$ & 41.786 & 108.026 & Lignite & Small & $\begin{array}{l}\text { Ba yin hu du ge } \\
\text { Reconnaissance } \\
\text { Area }\end{array}$ \\
\hline 46 & $\begin{array}{l}\text { Inner Mongolia } \\
\text { Autonomous Region }\end{array}$ & $\begin{array}{l}\text { Bayannur City } \\
\text { (Bayannao'er Shi) }\end{array}$ & $\begin{array}{l}\text { Urat Middle Banner } \\
\text { (Wulate Zhong Qi) }\end{array}$ & 41.725 & 108.086 & Lignite & Small & Ba yin hu du ge \\
\hline 47 & $\begin{array}{l}\text { Inner Mongolia } \\
\text { Autonomous Region }\end{array}$ & $\begin{array}{l}\text { Bayannur City } \\
\text { (Bayannao'er Shi) }\end{array}$ & $\begin{array}{l}\text { Urat Middle Banner } \\
\text { (Wulate Zhong Qi) }\end{array}$ & 41.478 & 108.232 & Bituminous & Small & Ang gen \\
\hline 48 & $\begin{array}{l}\text { Inner Mongolia } \\
\quad \text { Autonomous Region }\end{array}$ & $\begin{array}{l}\text { Hohot City } \\
\quad \text { (Huhehaote Shi) }\end{array}$ & $\begin{array}{l}\text { Wuchuan County } \\
\text { (Wuchuan Xian) }\end{array}$ & 41.363 & 110.801 & Lignite & Medium & $\begin{array}{l}\text { Liu tong hao } \\
\text { Well Field }\end{array}$ \\
\hline
\end{tabular}


Table 1. List of coal mines in China.-Continued

[Abbreviations used: dec. deg. - decimal degrees; NA - not available or not applicable]

\begin{tabular}{|c|c|c|c|c|c|c|c|c|}
\hline $\begin{array}{c}\text { ID } \\
\text { Number }\end{array}$ & $\begin{array}{c}\text { Province, National } \\
\text { Municipality, or } \\
\text { Autonomous Region }\end{array}$ & $\begin{array}{c}\text { Prefecture, Municipality, } \\
\text { Prefecture-Level City, or } \\
\text { League }\end{array}$ & $\begin{array}{l}\text { County, District or } \\
\text { County-Level City }\end{array}$ & $\begin{array}{l}\text { Latitude } \\
\text { (dec. deg.) }\end{array}$ & $\begin{array}{l}\text { Longitude } \\
\text { (dec. deg.) }\end{array}$ & Rank & $\begin{array}{l}\text { Relative } \\
\text { Mine Size }\end{array}$ & Mine Name \\
\hline 49 & $\begin{array}{l}\text { Inner Mongolia } \\
\text { Autonomous Region }\end{array}$ & $\begin{array}{l}\text { Hohot City } \\
\quad \text { (Huhehaote Shi) }\end{array}$ & $\begin{array}{l}\text { Wuchuan County } \\
\text { (Wuchuan Xian) }\end{array}$ & 41.211 & 111.289 & Bituminous & Small & Da hai liu Well Field \\
\hline 50 & $\begin{array}{l}\text { Inner Mongolia } \\
\text { Autonomous Region }\end{array}$ & $\begin{array}{l}\text { Bayannur City } \\
\quad \text { (Bayannao'er Shi) }\end{array}$ & $\begin{array}{l}\text { Urat Middle Banner } \\
\text { (Wulate Zhong Qi) }\end{array}$ & 41.021 & 109.316 & Anthracite & Small & Mei yao gou \\
\hline 51 & $\begin{array}{l}\text { Inner Mongolia } \\
\text { Autonomous Region }\end{array}$ & $\begin{array}{l}\text { Bayannur City } \\
\text { (Bayannao'er Shi) }\end{array}$ & $\begin{array}{l}\text { Urat Middle Banner } \\
\text { (Wulate Zhong Qi) }\end{array}$ & 40.976 & 109.563 & Bituminous & Small & $\begin{array}{l}\text { Wu fen zi Qi fen zi } \\
\text { Exploration Area }\end{array}$ \\
\hline 52 & $\begin{array}{l}\text { Inner Mongolia } \\
\text { Autonomous Region }\end{array}$ & $\begin{array}{l}\text { Bayannur City } \\
\text { (Bayannao'er Shi) }\end{array}$ & $\begin{array}{l}\text { Urat Middle Banner } \\
\quad \text { (Wulate Zhong Qi) }\end{array}$ & 40.976 & 109.563 & Bituminous & Small & $\begin{array}{l}\text { Da luo gou } \\
\text { Exploration Area }\end{array}$ \\
\hline 53 & $\begin{array}{l}\text { Inner Mongolia } \\
\text { Autonomous Region }\end{array}$ & $\begin{array}{l}\text { Bayannur City } \\
\text { (Bayannao'er Shi) }\end{array}$ & $\begin{array}{l}\text { Urat Middle Banner } \\
\text { (Wulate Zhong Qi) }\end{array}$ & 40.976 & 109.563 & Bituminous & Small & $\begin{array}{l}\text { Yu sheng gong } \\
\text { Xi guan Well }\end{array}$ \\
\hline 54 & $\begin{array}{l}\text { Inner Mongolia } \\
\quad \text { Autonomous Region }\end{array}$ & $\begin{array}{l}\text { Bayannur City } \\
\quad \text { (Bayannao'er Shi) }\end{array}$ & $\begin{array}{l}\text { Urat Middle Banner } \\
\quad \text { (Wulate Zhong Qi) }\end{array}$ & 40.976 & 109.563 & Bituminous & Small & Ying pan wan \\
\hline 55 & $\begin{array}{l}\text { Inner Mongolia } \\
\text { Autonomous Region }\end{array}$ & $\begin{array}{l}\text { Bayannur City } \\
\quad \text { (Bayannao'er Shi) }\end{array}$ & $\begin{array}{l}\text { Urat Middle Banner } \\
\quad \text { (Wulate Zhong Qi) }\end{array}$ & 40.976 & 109.563 & Bituminous & Small & $\begin{array}{l}\text { Er fen zi Shi yi fen zi } \\
\text { Exploration Area }\end{array}$ \\
\hline 56 & $\begin{array}{l}\text { Inner Mongolia } \\
\quad \text { Autonomous Region }\end{array}$ & Baotou City (Baotou Shi) & $\begin{array}{l}\text { Guyang County } \\
\text { (Guyang Xian) }\end{array}$ & 41.153 & 109.857 & Lignite & Small & Wo er qin hao \\
\hline 57 & $\begin{array}{l}\text { Inner Mongolia } \\
\text { Autonomous Region }\end{array}$ & Baotou City (Baotou Shi) & $\begin{array}{l}\text { Guyang County } \\
\text { (Guyang Xian) }\end{array}$ & 41.174 & 110.193 & Lignite & Small & Jia ba \\
\hline 58 & $\begin{array}{l}\text { Inner Mongolia } \\
\text { Autonomous Region }\end{array}$ & Baotou City (Baotou Shi) & NA & 40.708 & 110.345 & Bituminous & Small & $\begin{array}{l}\text { Shi guai Mining Area, } \\
\text { Bai hu gou }\end{array}$ \\
\hline 59 & $\begin{array}{l}\text { Inner Mongolia } \\
\text { Autonomous Region }\end{array}$ & Baotou City (Baotou Shi) & NA & 40.705 & 110.409 & Bituminous & Small & Yang ge leng \\
\hline 60 & $\begin{array}{l}\text { Inner Mongolia } \\
\text { Autonomous Region }\end{array}$ & $\begin{array}{l}\text { Hohot City } \\
\text { (Huhehaote Shi) }\end{array}$ & $\begin{array}{r}\text { Tumed Left Banner } \\
\text { (Tumote Zuo Qi) }\end{array}$ & 40.746 & 110.633 & Anthracite & Small & Wan jia gou \\
\hline 61 & $\begin{array}{l}\text { Inner Mongolia } \\
\text { Autonomous Region }\end{array}$ & $\begin{array}{l}\text { Hohot City } \\
\quad \text { (Huhehaote Shi) }\end{array}$ & $\begin{array}{l}\text { Tumed Left Banner } \\
\text { (Tumote Zuo Qi) }\end{array}$ & 40.746 & 110.633 & Anthracite & Small & Da nan gou \\
\hline 62 & $\begin{array}{l}\text { Inner Mongolia } \\
\text { Autonomous Region }\end{array}$ & $\begin{array}{l}\text { Hohot City } \\
\text { (Huhehaote Shi) }\end{array}$ & $\begin{array}{l}\text { Tumed Left Banner } \\
\text { (Tumote Zuo Qi) }\end{array}$ & 40.754 & 110.634 & Anthracite & Small & Ye ma tu \\
\hline 63 & $\begin{array}{l}\text { Inner Mongolia } \\
\text { Autonomous Region }\end{array}$ & $\begin{array}{l}\text { Hohot City } \\
\text { (Huhehaote Shi) }\end{array}$ & $\begin{array}{r}\text { Tumed Left Banner } \\
\text { (Tumote Zuo Qi) }\end{array}$ & 40.754 & 110.634 & Anthracite & Small & Liu dan ba \\
\hline 64 & $\begin{array}{l}\text { Inner Mongolia } \\
\text { Autonomous Region }\end{array}$ & $\begin{array}{l}\text { Hohot City } \\
\quad \text { (Huhehaote Shi) }\end{array}$ & $\begin{array}{r}\text { Tumed Left Banner } \\
\text { (Tumote Zuo Qi) }\end{array}$ & 40.721 & 110.685 & Bituminous & Small & Zhong bu ge su \\
\hline 65 & $\begin{array}{l}\text { Inner Mongolia } \\
\quad \text { Autonomous Region }\end{array}$ & Baotou City (Baotou Shi) & NA & 40.700 & 110.281 & Bituminous & Small & Wei jun ba \\
\hline 66 & $\begin{array}{l}\text { Inner Mongolia } \\
\text { Autonomous Region }\end{array}$ & Baotou City (Baotou Shi) & NA & 40.700 & 110.281 & Bituminous & Small & Ji mao yao zi \\
\hline 67 & $\begin{array}{l}\text { Inner Mongolia } \\
\text { Autonomous Region }\end{array}$ & Baotou City (Baotou Shi) & NA & 40.671 & 110.337 & Bituminous & Small & $\begin{array}{l}\text { Shi guai minng Area, } \\
\text { Chang han gou }\end{array}$ \\
\hline
\end{tabular}


Table 1. List of coal mines in China.-Continued

[Abbreviations used: dec. deg. - decimal degrees; NA - not available or not applicable]

\begin{tabular}{|c|c|c|c|c|c|c|c|c|}
\hline $\begin{array}{l}\text { ID } \\
\text { Number }\end{array}$ & $\begin{array}{l}\text { Province, National } \\
\text { Municipality, or } \\
\text { Autonomous Region }\end{array}$ & $\begin{array}{c}\text { Prefecture, Municipality, } \\
\text { Prefecture-Level City, or } \\
\text { League }\end{array}$ & $\begin{array}{l}\text { County, District or } \\
\text { County-Level City }\end{array}$ & $\begin{array}{l}\text { Latitude } \\
\text { (dec. deg.) }\end{array}$ & $\begin{array}{l}\text { Longitude } \\
\text { (dec. deg.) }\end{array}$ & Rank & $\begin{array}{l}\text { Relative } \\
\text { Mine Size }\end{array}$ & Mine Name \\
\hline 68 & $\begin{array}{l}\text { Inner Mongolia } \\
\text { Autonomous Region }\end{array}$ & Baotou City (Baotou Shi) & NA & 40.671 & 110.337 & Bituminous & Small & $\begin{array}{l}\text { Shi guai minng Area, } \\
\text { He tan gou }\end{array}$ \\
\hline 69 & $\begin{array}{l}\text { Inner Mongolia } \\
\text { Autonomous Region }\end{array}$ & Baotou City (Baotou Shi) & NA & 40.671 & 110.337 & Bituminous & Small & $\begin{array}{l}\text { Shi guai minng Area, } \\
\text { Wu dong gou }\end{array}$ \\
\hline 70 & $\begin{array}{l}\text { Inner Mongolia } \\
\text { Autonomous Region }\end{array}$ & Baotou City (Baotou Shi) & NA & 40.671 & 110.337 & Bituminous & Small & $\begin{array}{l}\text { Shi guai minng Area, } \\
\text { Da ci }\end{array}$ \\
\hline 71 & $\begin{array}{l}\text { Inner Mongolia } \\
\text { Autonomous Region }\end{array}$ & Baotou City (Baotou Shi) & NA & 40.671 & 110.337 & Bituminous & Small & $\begin{array}{l}\text { Outskirts of } \\
\text { Hai liu shu }\end{array}$ \\
\hline 72 & $\begin{array}{l}\text { Inner Mongolia } \\
\text { Autonomous Region }\end{array}$ & Baotou City (Baotou Shi) & NA & 40.691 & 110.461 & Bituminous & Medium & $\begin{array}{l}\text { Shi guai Mining Area } \\
\text { A dao hai }\end{array}$ \\
\hline 73 & $\begin{array}{l}\text { Inner Mongolia } \\
\text { Autonomous Region }\end{array}$ & $\begin{array}{l}\text { Hohot City } \\
\text { (Huhehaote Shi) }\end{array}$ & $\begin{array}{r}\text { Tumed Left Banner } \\
\text { (Tumote Zuo Qi) }\end{array}$ & 40.756 & 111.154 & Anthracite & Small & Cha su qi \\
\hline 74 & $\begin{array}{l}\text { Inner Mongolia } \\
\text { Autonomous Region }\end{array}$ & $\begin{array}{l}\text { Xilingol League } \\
\text { (Xilinguole Meng) }\end{array}$ & $\begin{array}{l}\text { Sonid Right Banner } \\
\quad \text { (Sunite You Qi) }\end{array}$ & 42.616 & 112.682 & Lignite & Small & $\begin{array}{l}\text { Sai han ta la Coal } \\
\text { Exploration Area }\end{array}$ \\
\hline 75 & $\begin{array}{l}\text { Inner Mongolia } \\
\text { Autonomous Region }\end{array}$ & $\begin{array}{l}\text { Xilingol League } \\
\text { (Xilinguole Meng) }\end{array}$ & $\begin{array}{l}\text { Bordered Yellow } \\
\text { Banner } \\
\text { (Xianghuang Qi) }\end{array}$ & 42.200 & 114.355 & Anthracite & Small & Shi jiang shan \\
\hline 76 & $\begin{array}{l}\text { Inner Mongolia } \\
\text { Autonomous Region }\end{array}$ & $\begin{array}{l}\text { Xilingol League } \\
\text { (Xilinguole Meng) }\end{array}$ & $\begin{array}{l}\text { Plain Blue Banner } \\
\text { (Zhenlan Qi) }\end{array}$ & 42.075 & 115.880 & Lignite & Small & Hei cheng zi \\
\hline 77 & $\begin{array}{l}\text { Inner Mongolia } \\
\text { Autonomous Region }\end{array}$ & $\begin{array}{l}\text { Ulanqab City } \\
\text { (Wulanchabu Shi) }\end{array}$ & $\begin{array}{l}\text { Zhuozi County } \\
\text { (Zhuozi Xian) }\end{array}$ & 41.228 & 112.119 & Anthracite & Small & $\begin{array}{l}\text { No. } 4 \text { Exploration } \\
\text { Area }\end{array}$ \\
\hline 78 & $\begin{array}{l}\text { Inner Mongolia } \\
\text { Autonomous Region }\end{array}$ & $\begin{array}{l}\text { Ulanqab City } \\
\text { (Wulanchabu Shi) }\end{array}$ & $\begin{array}{l}\text { Chahar Right Middle } \\
\text { Banner (Chaha'er } \\
\text { Youyi Zhong Qi) }\end{array}$ & 41.178 & 112.345 & Bituminous & Small & Su le tu \\
\hline 79 & $\begin{array}{l}\text { Inner Mongolia } \\
\text { Autonomous Region }\end{array}$ & $\begin{array}{l}\text { Ulanqab City } \\
\text { (Wulanchabu Shi) }\end{array}$ & $\begin{array}{l}\text { Chahar Right Middle } \\
\text { Banner (Chaha'er } \\
\text { Youyi Zhong Qi) }\end{array}$ & 41.149 & 112.465 & Anthracite & Small & $\begin{array}{l}\text { Du ba } \\
\quad \text { Exploration Area }\end{array}$ \\
\hline 80 & $\begin{array}{l}\text { Inner Mongolia } \\
\text { Autonomous Region }\end{array}$ & $\begin{array}{l}\text { Ulanqab City } \\
\text { (Wulanchabu Shi) }\end{array}$ & $\begin{array}{l}\text { Jining District } \\
\quad(\text { Jining Qu) }\end{array}$ & 41.137 & 113.140 & Lignite & Small & Ma lian tan \\
\hline 81 & $\begin{array}{l}\text { Inner Mongolia } \\
\text { Autonomous Region }\end{array}$ & Chifeng City (Chifeng Shi) & $\begin{array}{l}\text { Linxi County } \\
\quad \text { (Linxi Xian) }\end{array}$ & 43.833 & 117.842 & Anthracite & Small & Lin $x i$ \\
\hline 82 & $\begin{array}{l}\text { Inner Mongolia } \\
\text { Autonomous Region }\end{array}$ & Chifeng City (Chifeng Shi) & $\begin{array}{l}\text { Bairin Right Banner } \\
\text { (Balin You Qi) }\end{array}$ & 43.734 & 118.666 & Anthracite & Small & Ta bu hua \\
\hline 83 & $\begin{array}{l}\text { Inner Mongolia } \\
\text { Autonomous Region }\end{array}$ & Chifeng City (Chifeng Shi) & $\begin{array}{l}\text { Hexigten Banner } \\
\quad \text { (Keshiketeng Qi) }\end{array}$ & 42.930 & 117.779 & Lignite & Small & Guang xing \\
\hline 84 & $\begin{array}{l}\text { Inner Mongolia } \\
\text { Autonomous Region }\end{array}$ & Chifeng City (Chifeng Shi) & $\begin{array}{l}\text { Ongniud Banner } \\
\text { (Wengniute Qi) }\end{array}$ & 42.743 & 118.136 & Lignite & Small & Song jia ying \\
\hline 85 & $\begin{array}{l}\text { Inner Mongolia } \\
\text { Autonomous Region }\end{array}$ & $\begin{array}{l}\text { Xilingol League } \\
\text { (Xilinguole Meng) }\end{array}$ & $\begin{array}{l}\text { Duolun County } \\
\text { (Duolun Xian) }\end{array}$ & 42.277 & 116.494 & Lignite & Small & Xi da cang \\
\hline
\end{tabular}


Table 1. List of coal mines in China.-Continued

[Abbreviations used: dec. deg. - decimal degrees; NA - not available or not applicable]

\begin{tabular}{|c|c|c|c|c|c|c|c|c|}
\hline $\begin{array}{l}\text { ID } \\
\text { Number }\end{array}$ & $\begin{array}{c}\text { Province, National } \\
\text { Municipality, or } \\
\text { Autonomous Region }\end{array}$ & $\begin{array}{l}\text { Prefecture, Municipality, } \\
\text { Prefecture-Level City, or } \\
\text { League }\end{array}$ & $\begin{array}{l}\text { County, District or } \\
\text { County-Level City }\end{array}$ & $\begin{array}{l}\text { Latitude } \\
\text { (dec. deg.) }\end{array}$ & $\begin{array}{l}\text { Longitude } \\
\text { (dec. deg.) }\end{array}$ & Rank & $\begin{array}{l}\text { Relative } \\
\text { Mine Size }\end{array}$ & Mine Name \\
\hline 86 & $\begin{array}{l}\text { Inner Mongolia } \\
\text { Autonomous Region }\end{array}$ & Chifeng City (Chifeng Shi) & NA & 42.472 & 119.159 & Lignite & Large & Yuan bao shan \\
\hline 87 & $\begin{array}{l}\text { Inner Mongolia } \\
\text { Autonomous Region }\end{array}$ & Chifeng City (Chifeng Shi) & NA & 42.535 & 119.588 & Lignite & Small & $\begin{array}{l}\text { Yuan bao shan Area, } \\
\text { Da feng shui gou }\end{array}$ \\
\hline 88 & $\begin{array}{l}\text { Inner Mongolia } \\
\text { Autonomous Region }\end{array}$ & Chifeng City (Chifeng Shi) & NA & 42.305 & 119.200 & Lignite & Small & $\begin{array}{l}\text { Yuan bao shan Area, } \\
\text { Hong miao }\end{array}$ \\
\hline 89 & $\begin{array}{l}\text { Inner Mongolia } \\
\text { Autonomous Region }\end{array}$ & Chifeng City (Chifeng Shi) & $\begin{array}{r}\text { Harqin Banner } \\
\text { (Kalaqin Qi) }\end{array}$ & 42.035 & 118.452 & Bituminous & Small & Qing feng \\
\hline 90 & $\begin{array}{l}\text { Inner Mongolia } \\
\text { Autonomous Region }\end{array}$ & Chifeng City (Chifeng Shi) & NA & 42.106 & 119.264 & Lignite & Small & $\begin{array}{l}\text { Yuan bao shan } \\
\text { Western Open Pit } \\
\text { Mine }\end{array}$ \\
\hline 91 & $\begin{array}{l}\text { Inner Mongolia } \\
\text { Autonomous Region }\end{array}$ & Chifeng City (Chifeng Shi) & NA & 42.106 & 119.264 & Lignite & Small & $\begin{array}{l}\text { Yuan bao shan Area, } \\
\text { Gu shan }\end{array}$ \\
\hline 92 & $\begin{array}{l}\text { Inner Mongolia } \\
\quad \text { Autonomous Region }\end{array}$ & Chifeng City (Chifeng Shi) & $\begin{array}{r}\text { Harqin Banner } \\
\text { (Kalaqin Qi) }\end{array}$ & 41.963 & 119.145 & Lignite & Small & $\mathrm{Xi}$ qiao \\
\hline 93 & $\begin{array}{l}\text { Inner Mongolia } \\
\text { Autonomous Region }\end{array}$ & Chifeng City (Chifeng Shi) & $\begin{array}{r}\text { Harqin Banner } \\
\text { (Kalaqin Qi) }\end{array}$ & 41.963 & 119.145 & Lignite & Small & San dao ying zi \\
\hline 94 & $\begin{array}{l}\text { Inner Mongolia } \\
\text { Autonomous Region }\end{array}$ & Chifeng City (Chifeng Shi) & NA & 41.963 & 119.145 & Lignite & Small & $\begin{array}{l}\text { Yuan bao shan, Area } \\
\text { Wu jia }\end{array}$ \\
\hline 95 & $\begin{array}{l}\text { Inner Mongolia } \\
\text { Autonomous Region }\end{array}$ & Chifeng City (Chifeng Shi) & Ningcheng County & 41.734 & 119.081 & Lignite & Small & Si long \\
\hline 96 & $\begin{array}{l}\text { Inner Mongolia } \\
\text { Autonomous Region }\end{array}$ & Chifeng City (Chifeng Shi) & $\begin{array}{l}\text { Ar Horqin Banner } \\
\text { (Aluke'erqin Qi) }\end{array}$ & 43.983 & 120.247 & Bituminous & Small & Wen du hua \\
\hline 100 & $\begin{array}{l}\text { Inner Mongolia } \\
\quad \text { Autonomous Region }\end{array}$ & $\begin{array}{l}\text { Tongliao City } \\
\text { (Tongliao Shi) }\end{array}$ & $\begin{array}{l}\text { Horqin Left Back } \\
\text { Banner (Ke'erqin } \\
\text { Zuoyi Hou Qi) }\end{array}$ & 43.252 & 123.546 & Bituminous & Small & $\begin{array}{l}\text { Shuang liao searching } \\
\text { Coal Exploration } \\
\text { Area }\end{array}$ \\
\hline 101 & $\begin{array}{l}\text { Inner Mongolia } \\
\text { Autonomous Region }\end{array}$ & Chifeng City (Chifeng Shi) & $\begin{array}{l}\text { Aohan Banner } \\
\text { (Aohan Qi) }\end{array}$ & 42.840 & 120.191 & Lignite & Small & Yu shu lin zi \\
\hline 105 & $\begin{array}{l}\text { Inner Mongolia } \\
\text { Autonomous Region }\end{array}$ & Chifeng City (Chifeng Shi) & $\begin{array}{l}\text { Aohan Banner } \\
\text { (Aohan Qi) }\end{array}$ & 42.356 & 120.733 & Bituminous & Small & Bao guo tu \\
\hline 106 & $\begin{array}{l}\text { Inner Mongolia } \\
\text { Autonomous Region }\end{array}$ & $\begin{array}{l}\text { Tongliao City } \\
\text { (Tongliao Shi) }\end{array}$ & $\begin{array}{l}\text { Naiman Banner } \\
\text { (Naiman Qi) }\end{array}$ & 42.417 & 121.094 & Bituminous & Small & Qing shui tang \\
\hline 107 & $\begin{array}{l}\text { Inner Mongolia } \\
\text { Autonomous Region }\end{array}$ & $\begin{array}{l}\text { Alxa League (Alashan } \\
\text { Meng) }\end{array}$ & $\begin{array}{l}\text { Alxa Left Banner } \\
\text { (Alashan You Qi) }\end{array}$ & 39.178 & 101.466 & Bituminous & Small & Xi da yao \\
\hline 108 & $\begin{array}{l}\text { Inner Mongolia } \\
\text { Autonomous Region }\end{array}$ & Wuhai City (Wuhai Shi) & NA & 39.777 & 106.912 & Bituminous & Small & Mu er gou \\
\hline 109 & $\begin{array}{l}\text { Inner Mongolia } \\
\text { Autonomous Region }\end{array}$ & Wuhai City (Wuhai Shi) & NA & 39.695 & 106.898 & Bituminous & Small & Ka bu qi \\
\hline
\end{tabular}


Table 1. List of coal mines in China.-Continued

[Abbreviations used: dec. deg. - decimal degrees; NA - not available or not applicable]

\begin{tabular}{|c|c|c|c|c|c|c|c|c|}
\hline $\begin{array}{c}\text { ID } \\
\text { Number }\end{array}$ & $\begin{array}{l}\text { Province, National } \\
\text { Municipality, or } \\
\text { Autonomous Region }\end{array}$ & $\begin{array}{c}\text { Prefecture, Municipality, } \\
\text { Prefecture-Level City, or } \\
\text { League }\end{array}$ & $\begin{array}{l}\text { County, District or } \\
\text { County-Level City }\end{array}$ & $\begin{array}{l}\text { Latitude } \\
\text { (dec. deg.) }\end{array}$ & $\begin{array}{l}\text { Longitude } \\
\text { (dec. deg.) }\end{array}$ & Rank & $\begin{array}{l}\text { Relative } \\
\text { Mine Size }\end{array}$ & Mine Name \\
\hline 110 & $\begin{array}{l}\text { Inner Mongolia } \\
\text { Autonomous Region }\end{array}$ & Wuhai City (Wuhai Shi) & NA & 39.602 & 106.635 & Bituminous & Small & Wu da wu hu shan \\
\hline 111 & $\begin{array}{l}\text { Inner Mongolia } \\
\text { Autonomous Region }\end{array}$ & Wuhai City (Wuhai Shi) & NA & 39.632 & 106.889 & Bituminous & Medium & $\begin{array}{l}\text { Luo tuo shan } \\
\text { Exploration Area }\end{array}$ \\
\hline 112 & $\begin{array}{l}\text { Inner Mongolia } \\
\text { Autonomous Region }\end{array}$ & Ordos City (E'erduosi Shi) & $\begin{array}{l}\text { Otog Banner } \\
\quad \text { (Etuoke Qi) }\end{array}$ & 39.603 & 107.077 & Bituminous & Small & $\begin{array}{l}\text { Zhuo zi shan, Dong lu } \\
\text { Coal Area }\end{array}$ \\
\hline 113 & $\begin{array}{l}\text { Inner Mongolia } \\
\text { Autonomous Region }\end{array}$ & Wuhai City (Wuhai Shi) & NA & 39.435 & 106.820 & Bituminous & Small & Lao shi dan \\
\hline 114 & $\begin{array}{l}\text { Inner Mongolia } \\
\text { Autonomous Region }\end{array}$ & Wuhai City (Wuhai Shi) & NA & 39.440 & 107.005 & Bituminous & Medium & $\begin{array}{l}\text { Di li bang wu su } \\
\text { Exploration Area }\end{array}$ \\
\hline 115 & $\begin{array}{l}\text { Inner Mongolia } \\
\quad \text { Autonomous Region }\end{array}$ & Ordos City (E'erduosi Shi) & $\begin{array}{l}\text { Otog Banner } \\
\quad \text { (Etuoke Qi) }\end{array}$ & 39.440 & 107.005 & Bituminous & Medium & Qi pan Well \\
\hline 116 & $\begin{array}{l}\text { Inner Mongolia } \\
\text { Autonomous Region }\end{array}$ & Ordos City (E'erduosi Shi) & $\begin{array}{l}\text { Otog Banner } \\
\quad \text { (Etuoke Qi) }\end{array}$ & 39.440 & 107.005 & Bituminous & Medium & $\begin{array}{l}\text { Zhuo zi shan, Dong lu } \\
\text { a er ba si }\end{array}$ \\
\hline 117 & $\begin{array}{l}\text { Inner Mongolia } \\
\text { Autonomous Region }\end{array}$ & Ordos City (E'erduosi Shi) & $\begin{array}{l}\text { Otog Banner } \\
\quad \text { (Etuoke Qi) }\end{array}$ & 39.440 & 107.005 & Bituminous & Medium & $\begin{array}{l}\text { Zhuo zi shan, Ku li } \\
\text { huo sha tu } \\
\text { Exploration Area }\end{array}$ \\
\hline 118 & $\begin{array}{l}\text { Inner Mongolia } \\
\text { Autonomous Region }\end{array}$ & Ordos City (E'erduosi Shi) & $\begin{array}{l}\text { Otog Banner } \\
\quad \text { (Etuoke Qi) }\end{array}$ & 39.440 & 107.005 & Bituminous & Medium & $\begin{array}{l}\text { Zhuo zi shan, } \\
\text { Qian li shan } \\
\text { Exploration Area }\end{array}$ \\
\hline 119 & $\begin{array}{l}\text { Inner Mongolia } \\
\text { Autonomous Region }\end{array}$ & Wuhai City (Wuhai Shi) & NA & 39.355 & 107.077 & Bituminous & Medium & $\begin{array}{l}\text { Bai yun wu su } \\
\text { Exploration Area }\end{array}$ \\
\hline 120 & $\begin{array}{l}\text { Inner Mongolia } \\
\text { Autonomous Region }\end{array}$ & Wuhai City (Wuhai Shi) & NA & 39.298 & 106.880 & Bituminous & Small & Gong wu su \\
\hline 121 & $\begin{array}{l}\text { Inner Mongolia } \\
\quad \text { Autonomous Region }\end{array}$ & $\begin{array}{l}\text { Alxa League } \\
\text { (Alashan Meng) }\end{array}$ & $\begin{array}{l}\text { Alxa Left Banner } \\
\quad \text { (Alashan Zuo Qi) }\end{array}$ & 39.121 & 106.025 & Bituminous & Small & Can te la \\
\hline 122 & $\begin{array}{l}\text { Inner Mongolia } \\
\text { Autonomous Region }\end{array}$ & $\begin{array}{l}\text { Alxa League } \\
\text { (Alashan Meng) }\end{array}$ & $\begin{array}{l}\text { Alxa Left Banner } \\
\quad \text { (Alashan Zuo Qi) }\end{array}$ & 39.086 & 106.062 & Anthracite & Small & Er dao ling \\
\hline 123 & $\begin{array}{l}\text { Inner Mongolia } \\
\text { Autonomous Region }\end{array}$ & Ordos City (E'erduosi Shi) & $\begin{array}{l}\text { Otog Banner } \\
\quad \text { (Etuoke Qi) }\end{array}$ & 38.275 & 106.551 & Bituminous & Small & $\begin{array}{l}\text { Chang cheng Coal } \\
\text { Mine, Western } \\
\text { Mining Area }\end{array}$ \\
\hline 124 & $\begin{array}{l}\text { Inner Mongolia } \\
\text { Autonomous Region }\end{array}$ & Ordos City (E'erduosi Shi) & $\begin{array}{l}\text { Dalad Banner } \\
\text { (Dalate Qi) }\end{array}$ & 40.007 & 109.760 & Bituminous & Small & Tang gong gou \\
\hline 125 & $\begin{array}{l}\text { Inner Mongolia } \\
\text { Autonomous Region }\end{array}$ & Ordos City (E'erduosi Shi) & $\begin{array}{l}\text { Dongsheng District } \\
\text { (Dongsheng Qu) }\end{array}$ & 39.923 & 109.875 & Bituminous & Medium & Han tai chuan \\
\hline 126 & $\begin{array}{l}\text { Inner Mongolia } \\
\text { Autonomous Region }\end{array}$ & Ordos City (E'erduosi Shi) & $\begin{array}{l}\text { Dongsheng District } \\
\text { (Dongsheng Qu) }\end{array}$ & 39.818 & 110.027 & Bituminous & Small & Dong sheng \\
\hline 127 & $\begin{array}{l}\text { Inner Mongolia } \\
\text { Autonomous Region }\end{array}$ & Ordos City (E'erduosi Shi) & $\begin{array}{l}\text { Dongsheng District } \\
\text { (Dongsheng Qu) }\end{array}$ & 39.779 & 110.291 & Bituminous & Small & Fu jia yang po \\
\hline
\end{tabular}


Table 1. List of coal mines in China.-Continued

[Abbreviations used: dec. deg. - decimal degrees; NA - not available or not applicable]

\begin{tabular}{|c|c|c|c|c|c|c|c|c|}
\hline $\begin{array}{c}\text { ID } \\
\text { Number }\end{array}$ & $\begin{array}{c}\text { Province, National } \\
\text { Municipality, or } \\
\text { Autonomous Region }\end{array}$ & $\begin{array}{c}\text { Prefecture, Municipality, } \\
\text { Prefecture-Level City, or } \\
\text { League }\end{array}$ & $\begin{array}{l}\text { County, District or } \\
\text { County-Level City }\end{array}$ & $\begin{array}{l}\text { Latitude } \\
\text { (dec. deg.) }\end{array}$ & $\begin{array}{l}\text { Longitude } \\
\text { (dec. deg.) }\end{array}$ & Rank & $\begin{array}{l}\text { Relative } \\
\text { Mine Size }\end{array}$ & Mine Name \\
\hline 128 & $\begin{array}{l}\text { Inner Mongolia } \\
\text { Autonomous Region }\end{array}$ & Ordos City (E'erduosi Shi) & $\begin{array}{c}\text { Dongsheng District } \\
\text { (Dongsheng Qu) }\end{array}$ & 39.661 & 109.300 & Bituminous & Medium & $\begin{array}{l}\text { Coal resources } \\
\text { Reconnaissance } \\
\text { Area }\end{array}$ \\
\hline 129 & $\begin{array}{l}\text { Inner Mongolia } \\
\text { Autonomous Region }\end{array}$ & Ordos City (E'erduosi Shi) & $\begin{array}{l}\text { Ejin Horo Banner } \\
\text { (Yijinhuoluo Qi) }\end{array}$ & 39.651 & 110.072 & Bituminous & Small & $\begin{array}{l}\text { Zhun ge er Mining } \\
\text { Area Hou shi ge tai } \\
\text { Detailed Survey } \\
\text { Area }\end{array}$ \\
\hline 130 & $\begin{array}{l}\text { Inner Mongolia } \\
\text { Autonomous Region }\end{array}$ & Ordos City (E'erduosi Shi) & $\begin{array}{l}\text { Ejin Horo Banner } \\
\text { (Yijinhuoluo Qi) }\end{array}$ & 39.586 & 110.241 & Bituminous & Small & Mu jiang gou \\
\hline 132 & $\begin{array}{l}\text { Inner Mongolia } \\
\text { Autonomous Region }\end{array}$ & Ordos City (E'erduosi Shi) & $\begin{array}{l}\text { Ejin Horo Banner } \\
\text { (Yijinhuoluo Qi) }\end{array}$ & 39.560 & 109.938 & Bituminous & Small & Ma jia ta \\
\hline 133 & $\begin{array}{l}\text { Inner Mongolia } \\
\text { Autonomous Region }\end{array}$ & Ordos City (E'erduosi Shi) & $\begin{array}{l}\text { Ejin Horo Banner } \\
\text { (Yijinhuoluo Qi) }\end{array}$ & 39.393 & 110.114 & Bituminous & Medium & $\mathrm{Bu}$ lian \\
\hline 135 & $\begin{array}{l}\text { Inner Mongolia } \\
\text { Autonomous Region }\end{array}$ & Ordos City (E'erduosi Shi) & $\begin{array}{l}\text { Jungar Banner } \\
\text { (Zhunge'er Qi) }\end{array}$ & 39.934 & 111.288 & Bituminous & Medium & $\begin{array}{l}\text { Dong kong dui } \\
\text { Prospective } \\
\text { Exploration Area }\end{array}$ \\
\hline 136 & $\begin{array}{l}\text { Inner Mongolia } \\
\text { Autonomous Region }\end{array}$ & Ordos City (E'erduosi Shi) & $\begin{array}{l}\text { Jungar Banner } \\
\text { (Zhunge'er Qi) }\end{array}$ & 39.934 & 111.288 & Bituminous & Medium & Shi yao gou \\
\hline 137 & $\begin{array}{l}\text { Inner Mongolia } \\
\text { Autonomous Region }\end{array}$ & Ordos City (E'erduosi Shi) & $\begin{array}{l}\text { Jungar Banner } \\
\quad \text { (Zhunge'er Qi) }\end{array}$ & 39.878 & 111.415 & Bituminous & Small & $\begin{array}{l}\text { Yao gou Open Pit } \\
\text { Accurately } \\
\text { Surveyed Area }\end{array}$ \\
\hline 138 & $\begin{array}{l}\text { Inner Mongolia } \\
\text { Autonomous Region }\end{array}$ & Ordos City (E'erduosi Shi) & $\begin{array}{l}\text { Jungar Banner } \\
\text { (Zhunge'er Qi) }\end{array}$ & 39.878 & 111.415 & Bituminous & Small & Niu lian gou \\
\hline 139 & $\begin{array}{l}\text { Inner Mongolia } \\
\text { Autonomous Region }\end{array}$ & Ordos City (E'erduosi Shi) & $\begin{array}{l}\text { Jungar Banner } \\
\quad \text { (Zhunge'er Qi) }\end{array}$ & 39.799 & 111.271 & Bituminous & Medium & $\begin{array}{l}\text { Long wang gou } \\
\text { Detailed } \\
\text { Exploration Area }\end{array}$ \\
\hline 140 & $\begin{array}{l}\text { Inner Mongolia } \\
\text { Autonomous Region }\end{array}$ & Ordos City (E'erduosi Shi) & $\begin{array}{l}\text { Jungar Banner } \\
\text { (Zhunge'er Qi) }\end{array}$ & 39.719 & 111.580 & Bituminous & Small & $\begin{array}{l}\text { Hei dai gou Open Pit } \\
\text { Accurately } \\
\text { Surveyed Area }\end{array}$ \\
\hline 141 & $\begin{array}{l}\text { Inner Mongolia } \\
\text { Autonomous Region }\end{array}$ & $\begin{array}{l}\text { Hohot City } \\
\text { (Huhehaote Shi) }\end{array}$ & $\begin{array}{l}\text { Qingshuihe County } \\
\text { (Qingshuihe Xian) }\end{array}$ & 39.713 & 111.336 & Bituminous & Medium & Ta er liang \\
\hline 142 & $\begin{array}{l}\text { Inner Mongolia } \\
\text { Autonomous Region }\end{array}$ & $\begin{array}{l}\text { Hohot City } \\
\text { (Huhehaote Shi) }\end{array}$ & $\begin{array}{l}\text { Qingshuihe County } \\
\text { (Qingshuihe Xian) }\end{array}$ & 39.713 & 111.336 & Bituminous & Medium & $\begin{array}{l}\text { Ji mu gou } \\
\text { Exploration Area }\end{array}$ \\
\hline 143 & $\begin{array}{l}\text { Inner Mongolia } \\
\text { Autonomous Region }\end{array}$ & $\begin{array}{l}\text { Hohot City } \\
\text { (Huhehaote Shi) }\end{array}$ & $\begin{array}{l}\text { Qingshuihe County } \\
\text { (Qingshuihe Xian) }\end{array}$ & 39.713 & 111.336 & Bituminous & Medium & Yao gou \\
\hline 144 & $\begin{array}{l}\text { Inner Mongolia } \\
\text { Autonomous Region }\end{array}$ & Ordos City (E'erduosi Shi) & $\begin{array}{l}\text { Jungar Banner } \\
\text { (Zhunge'er Qi) }\end{array}$ & 39.638 & 111.261 & Bituminous & Medium & $\begin{array}{l}\text { Southern outskirts of } \\
\text { Detailed Study Area }\end{array}$ \\
\hline 1 & Jiangsu Province & Xuzhou City & Feng County & 34.848 & 116.448 & Bituminous & Medium & Ding lou \\
\hline
\end{tabular}


Table 1. List of coal mines in China. - Continued

[Abbreviations used: dec. deg. - decimal degrees; NA - not available or not applicable]

\begin{tabular}{|c|c|c|c|c|c|c|c|c|}
\hline $\begin{array}{c}\text { ID } \\
\text { Number }\end{array}$ & $\begin{array}{c}\text { Province, National } \\
\text { Municipality, or } \\
\text { Autonomous Region }\end{array}$ & $\begin{array}{c}\text { Prefecture, Municipality, } \\
\text { Prefecture-Level City, or } \\
\text { League }\end{array}$ & $\begin{array}{l}\text { County, District or } \\
\text { County-Level City }\end{array}$ & $\begin{array}{l}\text { Latitude } \\
\text { (dec. deg.) }\end{array}$ & $\begin{array}{l}\text { Longitude } \\
\text { (dec. deg.) }\end{array}$ & Rank & $\begin{array}{l}\text { Relative } \\
\text { Mine Size }\end{array}$ & Mine Name \\
\hline 3 & Jiangsu Province & Xuzhou City & Feng County & 34.672 & 116.619 & Bituminous & Small & Da cheng zhuang \\
\hline 4 & Jiangsu Province & Xuzhou City & Feng County & 34.661 & 116.730 & Bituminous & Small & Hua shan \\
\hline 5 & Jiangsu Province & Xuzhou City & Feng County & 34.794 & 116.807 & Bituminous & Large & Zhang shuang lou \\
\hline 6 & Jiangsu Province & Xuzhou City & Pei County & 34.901 & 116.809 & Bituminous & Large & San he jian \\
\hline 7 & Jiangsu Province & Xuzhou City & Pei County & 34.901 & 116.809 & Bituminous & Large & $\mathrm{Xi}$ tao tun \\
\hline 8 & Jiangsu Province & Xuzhou City & Pei County & 34.901 & 116.809 & Bituminous & Large & Long gu \\
\hline 9 & Jiangsu Province & Xuzhou City & Pei County & 34.901 & 116.809 & Bituminous & Large & Long dong \\
\hline 10 & Jiangsu Province & Xuzhou City & Pei County & 34.677 & 116.871 & Bituminous & Medium & Pei qi \\
\hline 11 & Jiangsu Province & Xuzhou City & Pei County & 34.765 & 116.878 & Bituminous & Small & Shi lou \\
\hline 12 & Jiangsu Province & Xuzhou City & Pei County & 34.881 & 116.912 & Bituminous & Large & Yao qiao \\
\hline 13 & Jiangsu Province & Xuzhou City & Pei County & 34.725 & 116.927 & Bituminous & Small & Pei cheng \\
\hline 14 & Jiangsu Province & Xuzhou City & Pei County & 34.838 & 116.937 & Bituminous & Large & Da tun \\
\hline 15 & Jiangsu Province & Xuzhou City & Pei County & 34.784 & 116.954 & Bituminous & Medium & Kong zhuang \\
\hline 16 & Jiangsu Province & Xuzhou City & Pei County & 34.367 & 117.022 & Bituminous & Large & Zhang ji \\
\hline 17 & Jiangsu Province & Xuzhou City & Tongshan District & 34.178 & 117.078 & Bituminous & Medium & Wu zhuang \\
\hline 18 & Jiangsu Province & Xuzhou City & Tongshan District & 34.283 & 117.027 & Bituminous & Medium & Zhang jing \\
\hline 19 & Jiangsu Province & Xuzhou City & Tongshan District & 34.215 & 117.129 & Bituminous & Medium & Xin he \\
\hline 20 & Jiangsu Province & Xuzhou City & Tongshan District & 34.520 & 117.069 & Bituminous & Medium & Ma po \\
\hline 21 & Jiangsu Province & Xuzhou City & Tongshan District & 34.315 & 117.092 & Bituminous & Medium & Jia he \\
\hline 22 & Jiangsu Province & Xuzhou City & Tongshan District & 34.352 & 117.091 & Bituminous & Medium & Zhang xiao lou \\
\hline 23 & Jiangsu Province & Xuzhou City & Tongshan District & 34.352 & 117.091 & Bituminous & Medium & Pang zhuang \\
\hline 24 & Jiangsu Province & Xuzhou City & Tongshan District & 34.407 & 117.129 & Bituminous & Medium & \\
\hline 25 & Jiangsu Province & Xuzhou City & Tongshan District & 34.407 & 117.129 & Bituminous & Medium & Liu xin \\
\hline 26 & Jiangsu Province & Xuzhou City & Tongshan District & 34.109 & 117.154 & Bituminous & Small & San bao \\
\hline 27 & Jiangsu Province & Xuzhou City & NA & 34.225 & 117.232 & Bituminous & Small & Qi li gou \\
\hline 28 & Jiangsu Province & Xuzhou City & Tongshan District & 34.305 & 117.332 & Bituminous & Medium & Da huang shan \\
\hline 29 & Jiangsu Province & Xuzhou City & Tongshan District & 34.408 & 117.349 & Bituminous & Small & Qing shan quan \\
\hline 30 & Jiangsu Province & Xuzhou City & Tongshan District & 34.349 & 117.355 & Bituminous & Medium & Quan tai \\
\hline 31 & Jiangsu Province & Xuzhou City & Tongshan District & 34.521 & 117.366 & Bituminous & Small & Li guo \\
\hline 32 & Jiangsu Province & Xuzhou City & Tongshan District & 34.338 & 117.405 & Bituminous & Medium & Qi shan \\
\hline 33 & Jiangsu Province & Xuzhou City & Tongshan District & 34.404 & 117.406 & Bituminous & Medium & Han qiao \\
\hline 34 & Jiangsu Province & Xuzhou City & Tongshan District & 34.404 & 117.406 & Bituminous & Medium & Xia qiao \\
\hline 35 & Jiangsu Province & Xuzhou City & Tongshan District & 34.351 & 117.463 & Bituminous & Medium & Dong zhuang \\
\hline 36 & Jiangsu Province & Xuzhou City & Tongshan District & 34.440 & 117.469 & Bituminous & Small & Xin qiao \\
\hline 37 & Jiangsu Province & Nanjing City & Jiangning District & 32.047 & 118.966 & Bituminous & Small & Quan tang \\
\hline 38 & Jiangsu Province & Nanjing City & NA & 32.115 & 118.976 & Bituminous & Small & Zhong shan \\
\hline 39 & Jiangsu Province & Nanjing City & Jiangning District & 32.081 & 119.022 & Bituminous & Small & Pai shan \\
\hline 40 & Jiangsu Province & Nanjing City & Jiangning District & 32.081 & 119.020 & Bituminous & Small & Hu shan \\
\hline 41 & Jiangsu Province & Zhenjiang City & Jurong City & 32.147 & 119.052 & Anthracite & Small & Bao hua shan \\
\hline
\end{tabular}


Table 1. List of coal mines in China. - Continued

[Abbreviations used: dec. deg. - decimal degrees; NA - not available or not applicable]

\begin{tabular}{|c|c|c|c|c|c|c|c|c|}
\hline $\begin{array}{c}\text { ID } \\
\text { Number }\end{array}$ & $\begin{array}{c}\text { Province, National } \\
\text { Municipality, or } \\
\text { Autonomous Region }\end{array}$ & $\begin{array}{c}\text { Prefecture, Municipality, } \\
\text { Prefecture-Level City, or } \\
\text { League }\end{array}$ & $\begin{array}{l}\text { County, District or } \\
\text { County-Level City }\end{array}$ & $\begin{array}{l}\text { Latitude } \\
\text { (dec. deg.) }\end{array}$ & $\begin{array}{l}\text { Longitude } \\
\text { (dec. deg.) }\end{array}$ & Rank & $\begin{array}{l}\text { Relative } \\
\text { Mine Size }\end{array}$ & Mine Name \\
\hline 42 & Jiangsu Province & Zhenjiang City & Jurong City & 32.147 & 119.052 & Anthracite & Small & Liu jia bian \\
\hline 43 & Jiangsu Province & Zhenjiang City & Jurong City & 32.094 & 119.162 & Bituminous & Small & Wan shan \\
\hline 44 & Jiangsu Province & Zhenjiang City & Dantu District & 32.072 & 119.324 & Bituminous & Small & Dong feng \\
\hline 45 & Jiangsu Province & Zhenjiang City & Dantu District & 32.072 & 119.324 & Bituminous & Small & Xiao li shan \\
\hline 46 & Jiangsu Province & Zhenjiang City & Dantu District & 32.133 & 119.371 & Anthracite & Small & Shi li chang shan \\
\hline 47 & Jiangsu Province & Zhenjiang City & Dantu District & 32.043 & 119.426 & Bituminous & Small & Gu dong \\
\hline 48 & Jiangsu Province & Zhenjiang City & Dantu District & 32.043 & 119.426 & Bituminous & Small & Fu niu shan \\
\hline 49 & Jiangsu Province & Yangzhou City & Jiangdu District & 32.454 & 119.557 & Bituminous & Small & Jiang dū \\
\hline 50 & Jiangsu Province & Taizhou City & JingJiang City & 32.050 & 120.311 & Bituminous & Small & Hu shan \\
\hline 51 & Jiangsu Province & Nanjing City & Gaochun District & 31.274 & 118.958 & Anthracite & Small & Hua shan \\
\hline 52 & Jiangsu Province & Nanjing City & Jiangning District & 31.930 & 118.978 & Anthracite & Small & Qing long shan \\
\hline 53 & Jiangsu Province & Nanjing City & Jiangning District & 31.985 & 118.990 & Bituminous & Small & Tuan shan \\
\hline 54 & Jiangsu Province & Zhenjiang City & Dantu District & 31.865 & 119.342 & Bituminous & Small & Hua shan \\
\hline 55 & Jiangsu Province & Changzhou City & Jintan City & 31.681 & 119.447 & Bituminous & Small & Tu shan \\
\hline 56 & Jiangsu Province & Changzhou City & Liyang City & 31.181 & 119.473 & Anthracite & Small & Song ling \\
\hline 57 & Jiangsu Province & Changzhou City & Liyang City & 31.527 & 119.562 & Bituminous & Small & Gan dong \\
\hline 58 & Jiangsu Province & Changzhou City & Liyang City & 31.196 & 119.562 & Bituminous & Small & Li shan \\
\hline 59 & Jiangsu Province & Changzhou City & Liyang City & 31.578 & 119.581 & Bituminous & Small & $\mathrm{Ru}$ lin \\
\hline 60 & Jiangsu Province & Changzhou City & Liyang City & 31.547 & 119.674 & Anthracite & Small & Shang huang \\
\hline 61 & Jiangsu Province & Wuxi City & Yixing City & 31.237 & 119.613 & Bituminous & Small & Yuan tian \\
\hline 62 & Jiangsu Province & Zhenjiang City & Danyang City & 31.884 & 119.670 & Bituminous & Small & Dao shu \\
\hline 63 & Jiangsu Province & Wuxi City & Yixing City & 31.311 & 119.672 & Bituminous & Small & Shan juan dong \\
\hline 64 & Jiangsu Province & Wuxi City & Yixing City & 31.415 & 119.756 & Bituminous & Small & Xiao zhang shu \\
\hline 65 & Jiangsu Province & Wuxi City & Yixing City & 31.415 & 119.756 & Bituminous & Small & Hong ta \\
\hline 66 & Jiangsu Province & Changzhou City & Wujin District & 31.874 & 119.774 & Bituminous & Small & Ben niu \\
\hline 67 & Jiangsu Province & Changzhou City & Wujin District & 31.742 & 119.783 & Bituminous & Small & Hou yu \\
\hline 68 & Jiangsu Province & Changzhou City & Wujin District & 31.742 & 119.783 & Bituminous & Small & Bu yi qiao \\
\hline 69 & Jiangsu Province & Wuxi City & Yixing City & 31.268 & 119.803 & Bituminous & Small & Chuan bu \\
\hline 70 & Jiangsu Province & Wuxi City & Yixing City & 31.268 & 119.803 & Bituminous & Small & Ren shu \\
\hline 71 & Jiangsu Province & Wuxi City & Yixing City & 31.268 & 119.803 & Bituminous & Small & Ba dong \\
\hline 72 & Jiangsu Province & Wuxi City & Yixing City & 31.242 & 119.870 & Bituminous & Small & Bai ni chang \\
\hline 73 & Jiangsu Province & Wuxi City & Yixing City & 31.754 & 120.105 & Bituminous & Small & Heng shan qiao \\
\hline 74 & Jiangsu Province & Wuxi City & Jiangyin City & 31.884 & 120.249 & Bituminous & Small & Jiang yin \\
\hline 75 & Jiangsu Province & Wuxi City & Jiangyin City & 31.844 & 120.293 & Bituminous & Small & Hua shan \\
\hline 78 & Jiangsu Province & Wuxi City & Jiangyin City & 31.902 & 120.294 & Bituminous & Small & Cheng jiang \\
\hline 79 & Jiangsu Province & Wuxi City & Jiangyin City & 31.729 & 120.395 & Bituminous & Small & Wen lin \\
\hline 80 & Jiangsu Province & Suzhou City & Wu County & 31.138 & 120.451 & Bituminous & Small & Dong shan \\
\hline 81 & Jiangsu Province & Suzhou City & Wu County & 31.163 & 120.483 & Bituminous & Small & Don shan bei bu \\
\hline 82 & Jiangsu Province & Suzhou City & Zhangjiagang City & 31.884 & 120.546 & Bituminous & Small & Sha zhou \\
\hline
\end{tabular}


Table 1. List of coal mines in China. - Continued

[Abbreviations used: dec. deg. - decimal degrees; NA - not available or not applicable]

\begin{tabular}{|c|c|c|c|c|c|c|c|c|}
\hline $\begin{array}{c}\text { ID } \\
\text { Number }\end{array}$ & $\begin{array}{c}\text { Province, National } \\
\text { Municipality, or } \\
\text { Autonomous Region }\end{array}$ & $\begin{array}{c}\text { Prefecture, Municipality, } \\
\text { Prefecture-Level City, or } \\
\text { League }\end{array}$ & $\begin{array}{l}\text { County, District or } \\
\text { County-Level City }\end{array}$ & $\begin{array}{l}\text { Latitude } \\
\text { (dec. deg.) }\end{array}$ & $\begin{array}{l}\text { Longitude } \\
\text { (dec. deg.) }\end{array}$ & Rank & $\begin{array}{l}\text { Relative } \\
\text { Mine Size }\end{array}$ & Mine Name \\
\hline 83 & Jiangsu Province & Suzhou City & Zhangjiagang City & 31.790 & 120.598 & Bituminous & Small & Tang qiao er qu \\
\hline 84 & Jiangsu Province & Suzhou City & Changshu City & 31.719 & 120.628 & Bituminous & Small & Mao jia qiao \\
\hline 85 & Jiangsu Province & Suzhou City & Zhangjiagang City & 31.811 & 120.680 & Bituminous & Small & Tan qiao yi qu \\
\hline 86 & Jiangsu Province & Suzhou City & Zhangjiagang City & 31.811 & 120.680 & Bituminous & Small & Miao qiao \\
\hline 1 & Jiangxi Province & Jiujiang City & Xiushui County & 29.168 & 114.584 & Bituminous & Small & Xi keng (Xi Pit) \\
\hline 13 & Jiangxi Province & Jiujiang City & Ruichang City & 29.578 & 115.422 & Anthracite & Small & Mo jia \\
\hline 14 & Jiangxi Province & Jiujiang City & Ruichang City & 29.531 & 115.453 & Bituminous & Small & Su jia long \\
\hline 15 & Jiangxi Province & Jiujiang City & Jiujiang County & 29.614 & 115.697 & Bituminous & Small & Xin tang bu \\
\hline 17 & Jiangxi Province & Jiujiang City & Wuning County & 29.516 & 115.088 & Bituminous & Small & Quan ping \\
\hline 18 & Jiangxi Province & Jiujiang City & Wuning County & 29.486 & 115.134 & Bituminous & Small & Keng bei \\
\hline 19 & Jiangxi Province & Jiujiang City & Ruichang City & 29.508 & 115.514 & Bituminous & Small & Yu jia qiao \\
\hline 22 & Jiangxi Province & Jiujiang City & De'an County & 29.438 & 115.482 & Bituminous & Small & Fu shan \\
\hline 44 & Jiangxi Province & Jingdezhen City & Leping County & 29.191 & 117.348 & Bituminous & Small & Yong shan qiao \\
\hline 48 & Jiangxi Province & Yichun City & Yifeng County & 28.435 & 114.975 & Bituminous & Small & Tang pu \\
\hline 49 & Jiangxi Province & Yichun City & Shanggao County & 28.278 & 114.987 & Bituminous & Small & Ji jong shan \\
\hline 50 & Jiangxi Province & Yichun City & Wanzai County & 28.236 & 114.490 & Bituminous & Small & San xing \\
\hline 61 & Jiangxi Province & Nanchang City & Xinjian County & 28.521 & 115.617 & Bituminous & Small & Zhu fang \\
\hline 65 & Jiangxi Province & Yichun City & Gao'an City & 28.047 & 115.134 & Anthracite & Small & Ying gang ling \\
\hline 66 & Jiangxi Province & Yichun City & Gao'an City & 28.221 & 115.338 & Bituminous & Small & Jun shan \\
\hline 67 & Jiangxi Province & Yichun City & Gao'an City & 28.275 & 115.431 & Anthracite & Small & Da wang shan \\
\hline 69 & Jiangxi Province & Yichun City & Gao'an City & 28.190 & 115.352 & Bituminous & Small & $\mathrm{Wu}$ ful ling \\
\hline 70 & Jiangxi Province & Yichun City & Gao'an City & 28.156 & 115.313 & Bituminous & Small & Tai yang wei \\
\hline 71 & Jiangxi Province & Yichun City & Gao'an City & 28.106 & 115.339 & Bituminous & Small & San qiao \\
\hline 72 & Jiangxi Province & Yichun City & Gao'an City & 28.156 & 115.523 & Bituminous & Small & E mei shan \\
\hline 73 & Jiangxi Province & Yichun City & Feng City & 28.238 & 115.670 & Bituminous & Small & Dong shen ling \\
\hline 74 & Jiangxi Province & Yichun City & Feng City & 28.238 & 115.670 & Bituminous & Small & Wu she li \\
\hline 75 & Jiangxi Province & Yichun City & Feng City & 28.238 & 115.670 & Bituminous & Small & Yun zhuang \\
\hline 76 & Jiangxi Province & Yichun City & Feng City & 28.287 & 115.678 & Bituminous & Small & Mei xian ling \\
\hline 77 & Jiangxi Province & Yichun City & Feng City & 28.283 & 115.746 & Bituminous & Small & Xian gu ling \\
\hline 82 & Jiangxi Province & Shangrao City & Poyang County & 28.965 & 116.958 & Bituminous & Small & Hong men kou \\
\hline 83 & Jiangxi Province & Shangrao City & Wannian County & 28.763 & 116.853 & Bituminous & Small & Bai ma \\
\hline 84 & Jiangxi Province & Shangrao City & Yugan County & 28.723 & 116.694 & Bituminous & Small & Yu gan \\
\hline 86 & Jiangxi Province & Nanchang City & Jin County & 28.467 & 116.509 & Bituminous & Small & Zhong ling \\
\hline 87 & Jiangxi Province & Shangrao City & Yugan County & 28.464 & 116.594 & Bituminous & Small & Run xi \\
\hline 91 & Jiangxi Province & Nanchang City & Jin County & 28.280 & 116.168 & Bituminous & Small & Mo gang shan \\
\hline 93 & Jiangxi Province & Yichun City & Feng City & 28.223 & 116.059 & Bituminous & Small & Guan tian \\
\hline 97 & Jiangxi Province & Jingdezhen City & Leping County & 28.937 & 117.041 & Bituminous & Small & Ming shan \\
\hline 99 & Jiangxi Province & Jingdezhen City & Leping County & 28.889 & 117.154 & Bituminous & Small & Qiao tou qiu \\
\hline 104 & Jiangxi Province & Yiyang City & $\mathrm{NA}$ & 28.732 & 117.390 & Anthracite & Small & Cao xi nei hong \\
\hline
\end{tabular}


Table 1. List of coal mines in China. - Continued

[Abbreviations used: dec. deg. - decimal degrees; NA - not available or not applicable]

\begin{tabular}{|c|c|c|c|c|c|c|c|c|}
\hline $\begin{array}{c}\text { ID } \\
\text { Number }\end{array}$ & $\begin{array}{l}\text { Province, National } \\
\text { Municipality, or } \\
\text { Autonomous Region }\end{array}$ & $\begin{array}{l}\text { Prefecture, Municipality, } \\
\text { Prefecture-Level City, or } \\
\text { League }\end{array}$ & $\begin{array}{l}\text { County, District or } \\
\text { County-Level City }\end{array}$ & $\begin{array}{c}\text { Latitude } \\
\text { (dec. deg.) }\end{array}$ & $\begin{array}{l}\text { Longitude } \\
\text { (dec. deg.) }\end{array}$ & Rank & $\begin{array}{l}\text { Relative } \\
\text { Mine Size }\end{array}$ & Mine Name \\
\hline 106 & Jiangxi Province & Yiyang City & NA & 28.673 & 117.484 & Anthracite & Small & Mei xi qiao \\
\hline 112 & Jiangxi Province & Shangrao City & Hengfeng County & 28.468 & 117.583 & Bituminous & Small & $\mathrm{Xi}$ shan wu \\
\hline 116 & Jiangxi Province & Xinyu City & $\begin{array}{l}\text { Fenyi County, } \\
\text { Qianshan Town }\end{array}$ & 28.230 & 117.563 & Bituminous & Small & $\mathrm{Xi}$ an \\
\hline 117 & Jiangxi Province & Xinyu City & $\begin{array}{l}\text { Fenyi County, } \\
\text { Qianshan Town }\end{array}$ & 28.174 & 117.717 & Anthracite & Small & Wu du \\
\hline 122 & Jiangxi Province & Shangrao City & $\mathrm{NA}$ & 28.319 & 117.980 & Bituminous & Small & Dong tian \\
\hline 125 & Jiangxi Province & Shangrao City & Yushan County & 28.687 & 118.382 & Bituminous & Small & Mao zhai \\
\hline 128 & Jiangxi Province & Shangrao City & NA & 28.530 & 118.142 & Bituminous & Small & Yan keng (Yan Pit) \\
\hline 134 & Jiangxi Province & Shangrao City & Guangfeng County & 28.469 & 118.309 & Bituminous & Small & Shuang shan \\
\hline 135 & Jiangxi Province & Shangrao City & NA & 28.339 & 118.057 & Bituminous & Small & Lu jing \\
\hline 136 & Jiangxi Province & Shangrao City & NA & 28.282 & 118.123 & Anthracite & Small & Hua ting \\
\hline 138 & Jiangxi Province & Pinxiang City & NA & 27.834 & 113.854 & Anthracite & Small & Yang qi shan \\
\hline 140 & Jiangxi Province & Pinxiang City & NA & 27.669 & 113.812 & Anthracite & Small & San tian \\
\hline 141 & Jiangxi Province & Pinxiang City & NA & 27.656 & 113.890 & Bituminous & Small & Bai yuan \\
\hline 144 & Jiangxi Province & Pinxiang City & NA & 27.639 & 113.802 & Anthracite & Small & Qing shan \\
\hline 145 & Jiangxi Province & Pinxiang City & NA & 27.601 & 113.755 & Bituminous & Small & Shang quan ling \\
\hline 146 & Jiangxi Province & Pinxiang City & NA & 27.617 & 113.740 & Bituminous & Small & Hu jia fang \\
\hline 147 & Jiangxi Province & Pinxiang City & NA & 27.625 & 113.777 & Anthracite & Small & Gu pi chong \\
\hline 151 & Jiangxi Province & Pinxiang City & NA & 27.620 & 113.907 & Bituminous & Small & An yuan \\
\hline 152 & Jiangxi Province & Pinxiang City & NA & 27.627 & 113.933 & Bituminous & Small & Gao keng (Gao Pit) \\
\hline 153 & Jiangxi Province & Pinxiang City & NA & 27.613 & 113.922 & Bituminous & Small & Pan jia chong \\
\hline 154 & Jiangxi Province & Pinxiang City & NA & 27.597 & 113.941 & Anthracite & Small & $\mathrm{Xu}$ jia fang \\
\hline 155 & Jiangxi Province & Pinxiang City & Lianhua County & 27.282 & 113.927 & Anthracite & Small & Zhi long nan \\
\hline 156 & Jiangxi Province & Pinxiang City & Lianhua County & 27.204 & 113.953 & Anthracite & Small & Nian lin \\
\hline 157 & Jiangxi Province & Pinxiang City & Lianhua County & 27.204 & 113.953 & Anthracite & Small & Chang bu \\
\hline 159 & Jiangxi Province & Pinxiang City & Lianhua County & 27.033 & 113.853 & Anthracite & Small & Jie hua long \\
\hline 160 & Jiangxi Province & Yichun City & NA & 27.924 & 114.294 & Anthracite & Small & Hong tang \\
\hline 162 & Jiangxi Province & Yichun City & NA & 27.929 & 114.477 & Anthracite & Small & Shang jiang kou \\
\hline 163 & Jiangxi Province & Xinyu City & Fenyi County & 27.914 & 114.680 & Anthracite & Small & Yang jiao \\
\hline 165 & Jiangxi Province & Yichun City & NA & 27.844 & 114.494 & Anthracite & Small & Feng keng (Feng Pit) \\
\hline 167 & Jiangxi Province & Xinyu City & NA & 27.941 & 114.849 & Anthracite & Small & Huang hua \\
\hline 168 & Jiangxi Province & Xinyu City & NA & 27.919 & 114.979 & Bituminous & Small & Hua gu shan \\
\hline 174 & Jiangxi Province & Ji'an City & Jishui County & 27.513 & 114.949 & Anthracite & Small & Yang jia \\
\hline 175 & Jiangxi Province & Ji'an City & Anfu County & 27.451 & 114.649 & Anthracite & Small & Bei hua shan \\
\hline 176 & Jiangxi Province & Ji'an City & Anfu County & 27.451 & 114.649 & Anthracite & Small & Da guang shan \\
\hline 178 & Jiangxi Province & Ji'an City & Anfu County & 27.387 & 114.752 & Anthracite & Small & Feng tian \\
\hline 179 & Jiangxi Province & Ji'an City & NA & 27.490 & 114.860 & Anthracite & Small & Tang dong \\
\hline 180 & Jiangxi Province & Pinxiang City & Lianhua County & 27.271 & 114.026 & Anthracite & Small & Xi yun shan \\
\hline
\end{tabular}


Table 1. List of coal mines in China. - Continued

[Abbreviations used: dec. deg. - decimal degrees; NA - not available or not applicable]

\begin{tabular}{|c|c|c|c|c|c|c|c|c|}
\hline $\begin{array}{c}\text { ID } \\
\text { Number }\end{array}$ & $\begin{array}{c}\text { Province, National } \\
\text { Municipality, or } \\
\text { Autonomous Region }\end{array}$ & $\begin{array}{c}\text { Prefecture, Municipality, } \\
\text { Prefecture-Level City, or } \\
\text { League }\end{array}$ & $\begin{array}{l}\text { County, District or } \\
\text { County-Level City }\end{array}$ & $\begin{array}{l}\text { Latitude } \\
\text { (dec. deg.) }\end{array}$ & $\begin{array}{l}\text { Longitude } \\
\text { (dec. deg.) }\end{array}$ & Rank & $\begin{array}{l}\text { Relative } \\
\text { Mine Size }\end{array}$ & Mine Name \\
\hline 183 & Jiangxi Province & Ji'an City & NA & 27.059 & 114.505 & Bituminous & Small & Tian he \\
\hline 185 & Jiangxi Province & Yichun City & Feng City & 27.973 & 115.848 & Bituminous & Small & Luo shi \\
\hline 187 & Jiangxi Province & Ji'an City & Jishui County & 27.482 & 115.266 & Bituminous & Small & Tai shan \\
\hline 189 & Jiangxi Province & Ji'an City & Jishui County & 27.076 & 115.561 & Bituminous & Small & Luo tian \\
\hline 190 & Jiangxi Province & Ji'an City & Yongfeng County & 27.057 & 115.661 & Anthracite & Small & Xing yan \\
\hline 193 & Jiangxi Province & Fuzhou City & Chongren County & 27.643 & 116.151 & Bituminous & Small & Li po \\
\hline 203 & Jiangxi Province & Ganzhou Municipality & Xinfeng County & 25.131 & 114.924 & Anthracite & Small & Tie shi kou \\
\hline 205 & Jiangxi Province & Ganzhou Municipality & Ruijin City & 25.960 & 115.865 & Anthracite & Small & Qi bao \\
\hline 206 & Jiangxi Province & Ganzhou Municipality & Yudu County & 25.859 & 115.394 & Bituminous & Small & Li cun \\
\hline 209 & Jiangxi Province & Ganzhou Municipality & Huichang County & 25.821 & 115.747 & Anthracite & Small & Luo kou \\
\hline 211 & Jiangxi Province & Ganzhou Municipality & Yudu County & 25.724 & 115.308 & Anthracite & Small & Qi lu shan \\
\hline 213 & Jiangxi Province & Ganzhou Municipality & Gan County & 25.533 & 115.244 & Anthracite & Small & Xiao pen \\
\hline 214 & Jiangxi Province & Ganzhou Municipality & Xinfeng County & 25.346 & 115.169 & Anthracite & Small & Da qiao \\
\hline 216 & Jiangxi Province & Ganzhou Municipality & Longnan County & 24.872 & 114.801 & Anthracite & Small & Long nan \\
\hline 217 & Jiangxi Province & Ganzhou Municipality & Quannan County & 24.768 & 114.470 & Anthracite & Small & Zhang po \\
\hline 1 & Jilin Province & Bai City & Taonan City & 45.779 & 121.823 & Bituminous & Small & $\begin{array}{l}\text { Wan hong Coal Field } \\
\text { Wan bao }\end{array}$ \\
\hline 7 & Jilin Province & Changchun City & Jiutai City & 44.172 & 125.867 & Bituminous & Small & $\begin{array}{l}\text { Ying cheng Coal Field } \\
\text { Jiu tai }\end{array}$ \\
\hline 8 & Jilin Province & Changchun City & Jiutai City & 44.151 & 125.943 & Bituminous & Small & $\begin{array}{l}\text { Ying cheng Coal Field } \\
\text { Ying cheng }\end{array}$ \\
\hline 9 & Jilin Province & Jilin City & Shutan City & 44.192 & 126.576 & Lignite & Small & Hong yang \\
\hline 10 & Jilin Province & Jilin City & Shutan City & 44.269 & 126.658 & Lignite & Small & Si jian fang \\
\hline 11 & Jilin Province & Jilin City & Shutan City & 44.207 & 126.671 & Lignite & Small & Gang yao \\
\hline 12 & Jilin Province & Jilin City & Shutan City & 44.233 & 126.690 & Lignite & Small & Gang yao \\
\hline 13 & Jilin Province & Jilin City & Shutan City & 44.283 & 126.776 & Lignite & Small & Feng guang \\
\hline 14 & Jilin Province & Jilin City & Shutan City & 44.322 & 126.819 & Lignite & Small & Ji shu \\
\hline 15 & Jilin Province & Jilin City & Shutan City & 44.355 & 126.880 & Lignite & Small & Dong fu \\
\hline 16 & Jilin Province & Jilin City & Shutan City & 44.392 & 126.933 & Lignite & Small & Shu lan jie \\
\hline 17 & Jilin Province & Jilin City & Shutan City & 44.440 & 126.995 & Lignite & Small & Shui qu liu \\
\hline 19 & Jilin Province & Jilin City & Shutan City & 44.501 & 127.087 & Lignite & Small & $\begin{array}{l}\text { Shui qu liu Ping an } \\
\text { Reconnaissance } \\
\text { Survey Area }\end{array}$ \\
\hline 20 & Jilin Province & Jilin City & Shutan City & 44.556 & 127.167 & Lignite & Small & $\begin{array}{l}\text { Ping an } \\
\quad \text { Reconnaissance } \\
\text { Survey Area }\end{array}$ \\
\hline 21 & Jilin Province & Siping City & Lishu County & 43.266 & 124.663 & Bituminous & Small & Meng jia ling \\
\hline 22 & Jilin Province & Siping City & Gongzhuling City & 43.585 & 124.936 & Lignite & Small & Liu fang zi \\
\hline
\end{tabular}


Table 1. List of coal mines in China. - Continued

[Abbreviations used: dec. deg. - decimal degrees; NA - not available or not applicable]

\begin{tabular}{|c|c|c|c|c|c|c|c|c|}
\hline $\begin{array}{c}\text { ID } \\
\text { Number }\end{array}$ & $\begin{array}{l}\text { Province, National } \\
\text { Municipality, or } \\
\text { Autonomous Region }\end{array}$ & $\begin{array}{c}\text { Prefecture, Municipality, } \\
\text { Prefecture-Level City, or } \\
\text { League }\end{array}$ & $\begin{array}{l}\text { County, District or } \\
\text { County-Level City }\end{array}$ & $\begin{array}{c}\text { Latitude } \\
\text { (dec. deg.) }\end{array}$ & $\begin{array}{l}\text { Longitude } \\
\text { (dec. deg.) }\end{array}$ & Rank & $\begin{array}{l}\text { Relative } \\
\text { Mine Size }\end{array}$ & Mine Name \\
\hline 23 & Jilin Province & Liaoyuan City & NA & 43.041 & 124.932 & Bituminous & Small & $\begin{array}{l}\text { Liao yuan Coal Field } \\
\text { Da shui gang }\end{array}$ \\
\hline 24 & Jilin Province & Siping City & $\begin{array}{l}\text { Yitong Autonomous } \\
\text { County }\end{array}$ & 43.287 & 125.109 & Lignite & Small & Da hu shan \\
\hline 25 & Jilin Province & Changchun City & NA & 43.870 & 125.436 & Bituminous & Small & Shi bei ling \\
\hline 26 & Jilin Province & Changchun City & NA & 43.837 & 125.410 & Bituminous & Small & Jing yue tan \\
\hline 27 & Jilin Province & Changchun City & NA & 43.777 & 125.418 & Bituminous & Small & Xin li cheng \\
\hline 28 & Jilin Province & Changchun City & NA & 43.717 & 125.423 & Bituminous & Small & $\begin{array}{l}\text { Xin li cheng No. } 2 \\
\text { Coal Mine }\end{array}$ \\
\hline 29 & Jilin Province & Liaoyuan City & NA & 43.021 & 125.500 & Bituminous & Small & Ye zhu gou \\
\hline 30 & Jilin Province & Changchun City & NA & 43.980 & 125.599 & Bituminous & Small & $\begin{array}{l}\text { Yang cao gou Coal } \\
\text { Field, Yang cao gou }\end{array}$ \\
\hline 31 & Jilin Province & Changchun City & Shuangyang District & 43.409 & 125.699 & Bituminous & Small & $\begin{array}{l}\text { Shuang yang Coal } \\
\text { Field, Er dao }\end{array}$ \\
\hline 32 & Jilin Province & Changchun City & Shuangyang District & 43.421 & 125.832 & Bituminous & Small & $\begin{array}{l}\text { Shuang yang Coal } \\
\text { Field, Shuang dong }\end{array}$ \\
\hline 33 & Jilin Province & Changchun City & Shuangyang District & 43.381 & 125.915 & Anthracite & Small & $\begin{array}{l}\text { Shuang yang Coal } \\
\text { Field Ba mian shi }\end{array}$ \\
\hline 34 & Jilin Province & Changchun City & Shuangyang District & 43.322 & 125.948 & Anthracite & Small & $\begin{array}{l}\text { Shuang yang Coal } \\
\text { Field Yan tong shan }\end{array}$ \\
\hline 35 & Jilin Province & Jilin City & Huadian City & 43.138 & 126.606 & Bituminous & Small & Yu mu qiao zi \\
\hline 36 & Jilin Province & Jilin City & Jiaohe City & 43.713 & 127.416 & Bituminous & Small & Jiao he \\
\hline 37 & Jilin Province & Jilin City & Jiaohe City & 43.713 & 127.416 & Bituminous & Small & Jiao he \\
\hline 38 & Jilin Province & $\begin{array}{l}\text { Yanbian Autonomous } \\
\text { Prefecture }\end{array}$ & Dunhua City & 43.627 & 128.231 & Bituminous & Small & $\begin{array}{l}\text { Dun hua } \\
\text { Reconnaissance } \\
\text { Survey Area }\end{array}$ \\
\hline 39 & Jilin Province & $\begin{array}{l}\text { Yanbian Autonomous } \\
\text { Prefecture }\end{array}$ & Yanji City & 43.261 & 129.277 & Bituminous & Small & San dao \\
\hline 40 & Jilin Province & $\begin{array}{l}\text { Yanbian Autonomous } \\
\text { Prefecture }\end{array}$ & Wangqing County & 43.656 & 129.477 & Bituminous & Small & Chun yang \\
\hline 42 & Jilin Province & Liaoyuan City & NA & 42.944 & 124.880 & Bituminous & Small & $\begin{array}{l}\text { Liao yuan Coal Field, } \\
\text { Central Coal Mine, } \\
\text { San shu jing }\end{array}$ \\
\hline 43 & Jilin Province & Liaoyuan City & NA & 42.877 & 124.881 & Bituminous & Small & $\begin{array}{c}\text { Liao yuan Coal Field, } \\
\text { Tai xin, Ping gang }\end{array}$ \\
\hline 44 & Jilin Province & Liaoyuan City & NA & 42.980 & 124.944 & Bituminous & Small & $\begin{array}{l}\text { Liao yuan Coal Field, } \\
\text { Xi Liu }\end{array}$ \\
\hline
\end{tabular}


Table 1. List of coal mines in China.-Continued

[Abbreviations used: dec. deg. - decimal degrees; NA - not available or not applicable]

\begin{tabular}{|c|c|c|c|c|c|c|c|c|}
\hline $\begin{array}{c}\text { ID } \\
\text { Number }\end{array}$ & $\begin{array}{l}\text { Province, National } \\
\text { Municipality, or } \\
\text { Autonomous Region }\end{array}$ & $\begin{array}{c}\text { Prefecture, Municipality, } \\
\text { Prefecture-Level City, or } \\
\text { League }\end{array}$ & $\begin{array}{l}\text { County, District or } \\
\text { County-Level City }\end{array}$ & $\begin{array}{l}\text { Latitude } \\
\text { (dec. deg.) }\end{array}$ & $\begin{array}{l}\text { Longitude } \\
\text { (dec. deg.) }\end{array}$ & Rank & $\begin{array}{l}\text { Relative } \\
\text { Mine Size }\end{array}$ & Mine Name \\
\hline 45 & Jilin Province & Liaoyuan City & NA & 42.967 & 125.026 & Bituminous & Small & $\begin{array}{l}\text { Liao yuan Coal Field, } \\
\text { Bei Liu } \\
\text { Exploration Area }\end{array}$ \\
\hline 46 & Jilin Province & Liaoyuan City & NA & 42.889 & 125.019 & Bituminous & Small & $\begin{array}{l}\text { Liao yuan Coal Field, } \\
\text { Bai quan, } \\
\text { Ji xian Area }\end{array}$ \\
\hline 47 & Jilin Province & Liaoyuan City & NA & 42.962 & 125.112 & Bituminous & Small & $\begin{array}{l}\text { Liao yuan Coal Field, } \\
\text { Tai xin Mine, } \\
\text { No. } 4 \text { Well }\end{array}$ \\
\hline 48 & Jilin Province & Liaoyuan City & NA & 42.950 & 125.162 & Bituminous & Small & $\begin{array}{l}\text { Liao yuan Coal } \\
\text { Field, Xian }\end{array}$ \\
\hline 49 & Jilin Province & Liaoyuan City & NA & 42.930 & 125.185 & Bituminous & Small & $\begin{array}{l}\text { Liao yuan Coal Field, } \\
\text { Bai quan, Gang yao }\end{array}$ \\
\hline 50 & Jilin Province & Liaoyuan City & Dongfeng County & 42.854 & 125.410 & Bituminous & Small & Liao he yuan \\
\hline 51 & Jilin Province & Liaoyuan City & Meihekou City & 42.378 & 125.511 & Bituminous & Small & $\begin{array}{l}\text { Mei he Coal Field No. } \\
7 \text { Well }\end{array}$ \\
\hline 52 & Jilin Province & Liaoyuan City & Meihekou City & 42.354 & 125.442 & Bituminous & Small & $\begin{array}{l}\text { Mei he Coal Field, } \\
\text { Liao yuan Mine }\end{array}$ \\
\hline 53 & Jilin Province & Liaoyuan City & Meihekou City & 42.414 & 125.525 & Bituminous & Small & $\begin{array}{l}\text { Mei he Coal Field, } \\
\text { Mei he }\end{array}$ \\
\hline 54 & Jilin Province & Liaoyuan City & Meihekou City & 42.427 & 125.618 & Bituminous & Small & $\begin{array}{l}\text { Mei he Coal Field, } \\
\text { Si ba shi Area }\end{array}$ \\
\hline 61 & Jilin Province & Bai City & NA & 42.025 & 126.582 & Bituminous & Small & Ba bao \\
\hline 62 & Jilin Province & Bai City & NA & 42.054 & 126.604 & Bituminous & Small & Wei tang \\
\hline 63 & Jilin Province & Jilin City & Huadian City & 42.895 & 126.629 & Bituminous & Small & $\begin{array}{l}\text { Hua dian Coal Field } \\
\text { Wu dao gou }\end{array}$ \\
\hline 64 & Jilin Province & Bai City & NA & 42.142 & 126.648 & Bituminous & Small & Dong feng \\
\hline 65 & Jilin Province & Jilin City & Huadian City & 42.996 & 126.713 & Lignite & Small & $\begin{array}{l}\text { Hua dian Coal Field } \\
\text { Xi tai zi }\end{array}$ \\
\hline 66 & Jilin Province & Jilin City & Huadian City & 42.983 & 126.822 & Lignite & Small & $\begin{array}{l}\text { Hua dian Coal Field } \\
\text { Hua dian } \\
\text { Reconnaissance } \\
\text { Survey Area }\end{array}$ \\
\hline 68 & Jilin Province & Bai City & NA & 42.063 & 126.883 & Bituminous & Small & Wan gou \\
\hline 69 & Jilin Province & Baishan City & Jingyu County & 42.452 & 126.996 & Bituminous & Small & San dao gou \\
\hline 70 & Jilin Province & Baishan City & $\mathrm{NA}$ & 42.095 & 127.089 & Bituminous & Small & $\mathrm{Da}$ an \\
\hline 71 & Jilin Province & Baishan City & NA & 42.054 & 127.136 & Anthracite & Small & Guan dao ling \\
\hline 72 & Jilin Province & Baishan City & NA & 42.010 & 127.147 & Anthracite & Small & Song shu zhen \\
\hline 73 & Jilin Province & Baishan City & NA & 42.031 & 127.209 & Bituminous & Small & Xiao ying zi \\
\hline
\end{tabular}


Table 1. List of coal mines in China.-Continued

[Abbreviations used: dec. deg. - decimal degrees; NA - not available or not applicable]

\begin{tabular}{|c|c|c|c|c|c|c|c|c|}
\hline $\begin{array}{c}\text { ID } \\
\text { Number }\end{array}$ & $\begin{array}{l}\text { Province, National } \\
\text { Municipality, or } \\
\text { Autonomous Region }\end{array}$ & $\begin{array}{c}\text { Prefecture, Municipality, } \\
\text { Prefecture-Level City, or } \\
\text { League }\end{array}$ & $\begin{array}{l}\text { County, District or } \\
\text { County-Level City }\end{array}$ & $\begin{array}{l}\text { Latitude } \\
\text { (dec. deg.) }\end{array}$ & $\begin{array}{l}\text { Longitude } \\
\text { (dec. deg.) }\end{array}$ & Rank & $\begin{array}{l}\text { Relative } \\
\text { Mine Size }\end{array}$ & Mine Name \\
\hline 74 & Jilin Province & $\begin{array}{l}\text { Yanbian Autonomous } \\
\text { Prefecture }\end{array}$ & Antu County & 42.679 & 128.132 & Bituminous & Small & Si cha zi \\
\hline 75 & Jilin Province & $\begin{array}{l}\text { Yanbian Autonomous } \\
\text { Prefecture }\end{array}$ & Helong City & 42.391 & 128.764 & Bituminous & Small & Sha jing gou \\
\hline 76 & Jilin Province & $\begin{array}{l}\text { Yanbian Autonomous } \\
\text { Prefecture }\end{array}$ & Helong City & 42.504 & 128.973 & Bituminous & Small & Huo long \\
\hline 77 & Jilin Province & $\begin{array}{l}\text { Yanbian Autonomous } \\
\text { Prefecture }\end{array}$ & Helong City & 42.635 & 129.018 & Bituminous & Small & $\mathrm{Wu}$ ming \\
\hline 78 & Jilin Province & $\begin{array}{l}\text { Yanbian Autonomous } \\
\text { Prefecture }\end{array}$ & Helong City & 42.581 & 129.135 & Bituminous & Small & $\begin{array}{l}\text { Huo long Coal Field } \\
\text { Yan bian }\end{array}$ \\
\hline 79 & Jilin Province & $\begin{array}{l}\text { Yanbian Autonomous } \\
\text { Prefecture }\end{array}$ & Longjing City & 42.565 & 129.367 & Bituminous & Small & Yong xin \\
\hline 80 & Jilin Province & $\begin{array}{l}\text { Yanbian Autonomous } \\
\text { Prefecture }\end{array}$ & Longjing City & 42.494 & 129.685 & Lignite & Small & San he Area \\
\hline 81 & Jilin Province & $\begin{array}{l}\text { Yanbian Autonomous } \\
\text { Prefecture }\end{array}$ & Longjing City & 42.684 & 129.722 & Lignite & Small & Kai shan tun \\
\hline 82 & Jilin Province & $\begin{array}{l}\text { Yanbian Autonomous } \\
\text { Prefecture }\end{array}$ & Hunchun City & 42.986 & 130.009 & Lignite & Small & Liang shui \\
\hline 83 & Jilin Province & $\begin{array}{l}\text { Yanbian Autonomous } \\
\text { Prefecture }\end{array}$ & Hunchun City & 42.912 & 130.312 & Lignite & Small & Ying an \\
\hline 84 & Jilin Province & $\begin{array}{l}\text { Yanbian Autonomous } \\
\text { Prefecture }\end{array}$ & Hunchun City & 42.856 & 130.313 & Lignite & Small & Cheng xi \\
\hline 85 & Jilin Province & $\begin{array}{l}\text { Yanbian Autonomous } \\
\text { Prefecture }\end{array}$ & Hunchun City & 42.921 & 130.357 & Lignite & Small & Ba lian cheng \\
\hline 86 & Jilin Province & $\begin{array}{l}\text { Yanbian Autonomous } \\
\text { Prefecture }\end{array}$ & Hunchun City & 42.782 & 130.299 & Bituminous & Small & $\begin{array}{l}\text { Ban shi No. } 1 \\
\text { Accuratley } \\
\text { Survey Area }\end{array}$ \\
\hline 87 & Jilin Province & $\begin{array}{l}\text { Yanbian Autonomous } \\
\text { Prefecture }\end{array}$ & Hunchun City & 42.805 & 130.388 & Lignite & Small & $\begin{array}{l}\text { Ban shi } \\
\text { Exploration Area }\end{array}$ \\
\hline 88 & Jilin Province & $\begin{array}{l}\text { Yanbian Autonomous } \\
\text { Prefecture }\end{array}$ & Hunchun City & 42.813 & 130.441 & Lignite & Small & Nan shan Well \\
\hline 89 & Jilin Province & $\begin{array}{l}\text { Yanbian Autonomous } \\
\text { Prefecture }\end{array}$ & Hunchun City & 42.855 & 130.461 & Lignite & Small & Wu jia zi \\
\hline 90 & Jilin Province & $\begin{array}{l}\text { Yanbian Autonomous } \\
\text { Prefecture }\end{array}$ & Hunchun City & 42.940 & 130.462 & Bituminous & Small & Luo tao he zi (tuo?) \\
\hline 91 & Jilin Province & $\begin{array}{l}\text { Yanbian Autonomous } \\
\text { Prefecture }\end{array}$ & Hunchun City & 42.886 & 130.591 & Lignite & Small & Miao ling \\
\hline 92 & Jilin Province & Baishan City & NA & 41.784 & 126.192 & Bituminous & Small & $\begin{array}{l}\text { Si dao jiang } \\
\text { Exploration Area }\end{array}$ \\
\hline
\end{tabular}


Table 1. List of coal mines in China. - Continued

[Abbreviations used: dec. deg. - decimal degrees; NA - not available or not applicable]

\begin{tabular}{|c|c|c|c|c|c|c|c|c|}
\hline $\begin{array}{c}\text { ID } \\
\text { Number }\end{array}$ & $\begin{array}{c}\text { Province, National } \\
\text { Municipality, or } \\
\text { Autonomous Region }\end{array}$ & $\begin{array}{c}\text { Prefecture, Municipality, } \\
\text { Prefecture-Level City, or } \\
\text { League }\end{array}$ & $\begin{array}{l}\text { County, District or } \\
\text { County-Level City }\end{array}$ & $\begin{array}{l}\text { Latitude } \\
\text { (dec. deg.) }\end{array}$ & $\begin{array}{l}\text { Longitude } \\
\text { (dec. deg.) }\end{array}$ & Rank & $\begin{array}{l}\text { Relative } \\
\text { Mine Size }\end{array}$ & Mine Name \\
\hline 93 & Jilin Province & Baishan City & NA & 41.734 & 126.181 & Bituminous & Small & $\begin{array}{l}\text { Tong hua tie chang } \mathrm{Xi} \\
\text { mei chang }\end{array}$ \\
\hline 94 & Jilin Province & Baishan City & NA & 41.807 & 126.260 & Bituminous & Small & Wu dao jiang \\
\hline 95 & Jilin Province & Baishan City & NA & 41.776 & 126.295 & Bituminous & Small & Tou dao gou \\
\hline 96 & Jilin Province & Baishan City & NA & 41.831 & 126.287 & Anthracite & Small & Dao qing \\
\hline 97 & Jilin Province & Baishan City & NA & 41.831 & 126.287 & Anthracite & Small & Dao qing \\
\hline 98 & Jilin Province & Baishan City & NA & 41.869 & 126.322 & Bituminous & Small & $\begin{array}{l}\text { Liu dao jiang } \\
\quad \text { Exploration Area }\end{array}$ \\
\hline 99 & Jilin Province & Baishan City & NA & 41.878 & 126.390 & Bituminous & Small & Ba dao jiang \\
\hline 100 & Jilin Province & Baishan City & NA & 41.913 & 126.476 & Bituminous & Small & $\begin{array}{l}\text { Lao fang zi gou } \\
\text { li Area }\end{array}$ \\
\hline 101 & Jilin Province & Baishan City & NA & 41.930 & 126.567 & Bituminous & Small & Shi ren \\
\hline 102 & Jilin Province & Baishan City & NA & 41.992 & 126.542 & Bituminous & Small & Zha zi \\
\hline 103 & Jilin Province & Baishan City & NA & 41.774 & 127.060 & Bituminous & Small & Da hu \\
\hline 104 & Jilin Province & Baishan City & $\begin{array}{l}\text { Changbai Autonomous } \\
\text { County }\end{array}$ & 41.465 & 128.095 & Anthracite & Small & Shi ba dao gou \\
\hline 105 & Jilin Province & Baishan City & NA & 41.398 & 128.125 & Anthracite & Small & Yan jiang \\
\hline 1 & Liaoning Province & Chaoyang City & Jianping County & 42.148 & 119.752 & Bituminous & Small & Jian ping, Gan jia zi \\
\hline 2 & Liaoning Province & Chaoyang City & Beipiao City & 42.134 & 120.929 & Bituminous & Small & $\begin{array}{l}\text { Bei piao, } \\
\text { Huo shang gou }\end{array}$ \\
\hline 3 & Liaoning Province & Fuxin City & NA & 42.078 & 121.815 & Bituminous & Small & Xin qiu \\
\hline 4 & Liaoning Province & Fuxin City & NA & 42.064 & 121.796 & Bituminous & Medium & $\begin{array}{l}\text { Xin qiu Open Pit } \\
\text { Coal Mine }\end{array}$ \\
\hline 5 & Liaoning Province & Shenyang City & Kangping County & 42.693 & 122.955 & Bituminous & Medium & Kang bei \\
\hline 6 & Liaoning Province & Fuxin City & Zhangwu County & 42.601 & 122.368 & Bituminous & Small & Xie lin tai Lei jia are \\
\hline 8 & Liaoning Province & Shenyang City & Kangping County & 42.676 & 123.406 & Bituminous & Small & San tai zi \\
\hline 9 & Liaoning Province & Shenyang City & Kangping County & 42.639 & 123.364 & Bituminous & Small & Kang ping \\
\hline 10 & Liaoning Province & Shenyang City & Kangping County & 42.639 & 123.364 & Bituminous & Large & Bian jia \\
\hline 11 & Liaoning Province & Tieling City & $\begin{array}{l}\text { Diaobingshan City } \\
\quad \text { (formerly Tiefa City) }\end{array}$ & 42.443 & 123.581 & Bituminous & Small & Shi huang di \\
\hline 12 & Liaoning Province & Tieling City & $\begin{array}{l}\text { Diaobingshan City } \\
\quad \text { (formerly Tiefa City) }\end{array}$ & 42.419 & 123.559 & Bituminous & Small & Da xing li western \\
\hline 13 & Liaoning Province & Tieling City & $\begin{array}{l}\text { Diaobingshan City } \\
\text { (formerly Tiefa City) }\end{array}$ & 42.405 & 123.589 & Bituminous & Large & Tie fa \\
\hline 14 & Liaoning Province & Fushun City & $\mathrm{NA}$ & 42.035 & 124.068 & Bituminous & Small & Qing shi ling \\
\hline 16 & Liaoning Province & Chaoyang City & Beipiao City & 41.814 & 120.778 & Bituminous & Large & \\
\hline 17 & Liaoning Province & Chaoyang City & Beipiao City & 41.670 & 120.586 & Bituminous & Small & Bai yao \\
\hline 18 & Liaoning Province & Chaoyang City & NA & 41.630 & 120.363 & Bituminous & Small & Chao yang \\
\hline 19 & Liaoning Province & Jinzhou City & Linghai City & 41.233 & 120.766 & Bituminous & Small & \\
\hline
\end{tabular}


Table 1. List of coal mines in China. - Continued

[Abbreviations used: dec. deg. - decimal degrees; NA - not available or not applicable]

\begin{tabular}{|c|c|c|c|c|c|c|c|c|}
\hline $\begin{array}{c}\text { ID } \\
\text { Number }\end{array}$ & $\begin{array}{l}\text { Province, National } \\
\text { Municipality, or } \\
\text { Autonomous Region }\end{array}$ & $\begin{array}{l}\text { Prefecture, Municipality, } \\
\text { Prefecture-Level City, or } \\
\text { League }\end{array}$ & $\begin{array}{l}\text { County, District or } \\
\text { County-Level City }\end{array}$ & $\begin{array}{l}\text { Latitude } \\
\text { (dec. deg.) }\end{array}$ & $\begin{array}{l}\text { Longitude } \\
\text { (dec. deg.) }\end{array}$ & Rank & $\begin{array}{l}\text { Relative } \\
\text { Mine Size }\end{array}$ & Mine Name \\
\hline 20 & Liaoning Province & $\begin{array}{l}\text { Huludao City } \\
\text { (formerly Jinxi City) }\end{array}$ & NA & 41.061 & 120.618 & Bituminous & Large & Nan piao \\
\hline 21 & Liaoning Province & $\begin{array}{l}\text { Huludao City } \\
\text { (formerly Jinxi City) }\end{array}$ & NA & 41.014 & 120.827 & Bituminous & Medium & Jin zhou Ha ma shan \\
\hline 22 & Liaoning Province & Fuxin City & NA & 41.996 & 121.691 & Bituminous & Large & Gao de \\
\hline 23 & Liaoning Province & Fuxin City & NA & 41.996 & 121.691 & Bituminous & Large & Hai zhou \\
\hline 24 & Liaoning Province & Fuxin City & NA & 41.993 & 121.958 & Bituminous & Medium & Wu long \\
\hline 25 & Liaoning Province & Fuxin City & NA & 41.950 & 121.610 & Bituminous & Medium & Ping an \\
\hline 26 & Liaoning Province & Fuxin City & NA & 41.915 & 121.628 & Bituminous & Medium & Wang jia ying zi \\
\hline 27 & Liaoning Province & Fuxin City & NA & 41.879 & 121.591 & Bituminous & Medium & Shuang shan \\
\hline 28 & Liaoning Province & Fuxin City & NA & 41.859 & 121.628 & Bituminous & Small & Dong liang \\
\hline 29 & Liaoning Province & Fuxin City & NA & 41.853 & 121.544 & Bituminous & Medium & Dong liang, Sha hai zu \\
\hline 30 & Liaoning Province & Jinzhou City & Heishan County & 41.812 & 121.987 & Bituminous & Medium & Ba dao gou \\
\hline 31 & Liaoning Province & Fuxin City & NA & 41.795 & 121.476 & Bituminous & Medium & Ai you \\
\hline 32 & Liaoning Province & Fuxin City & NA & 41.747 & 121.414 & Bituminous & Medium & Qing he men \\
\hline 33 & Liaoning Province & Shenyang City & Haicheng City & 41.086 & 122.645 & Anthracite & Small & Xin tai zi \\
\hline 34 & Liaoning Province & Liaoyang City & NA & 41.061 & 122.689 & Anthracite & Small & Xu zhu zi \\
\hline 35 & Liaoning Province & Shenyang City & NA & 41.982 & 123.424 & Lignite & Medium & Ma gu \\
\hline 36 & Liaoning Province & Shenyang City & NA & 41.971 & 123.438 & Lignite & Small & Jin bu \\
\hline 37 & Liaoning Province & Shenyang City & NA & 41.850 & 124.029 & Bituminous & Large & Long feng \\
\hline 38 & Liaoning Province & Fushun City & NA & 41.839 & 123.935 & Bituminous & Large & Lao hu tai \\
\hline 40 & Liaoning Province & Fushun City & NA & 41.837 & 123.845 & Bituminous & Large & Sheng li \\
\hline 41 & Liaoning Province & Fushun City & NA & 41.837 & 123.845 & Bituminous & Large & $\begin{array}{l}\text { Western Open Pit } \\
\text { Coal Mine }\end{array}$ \\
\hline 43 & Liaoning Province & Fushun City & NA & 41.826 & 123.570 & Bituminous & Large & Shen bei \\
\hline 44 & Liaoning Province & Fushun City & NA & 41.586 & 123.356 & Bituminous & Large & Shen nan \\
\hline 47 & Liaoning Province & Benxi City & NA & 41.336 & 123.919 & Anthracite & Small & Niu xin \\
\hline 48 & Liaoning Province & Benxi City & NA & 41.315 & 123.839 & Bituminous & Small & Da yu \\
\hline 50 & Liaoning Province & Benxi City & NA & 41.334 & 124.930 & Anthracite & Small & Nuan he zi \\
\hline 51 & Liaoning Province & Fushun City & $\begin{array}{l}\text { Xinbin Autonomous } \\
\text { County }\end{array}$ & 41.268 & 124.589 & Bituminous & Small & Xu jia da gou \\
\hline 52 & Liaoning Province & Fushun City & NA & 41.265 & 124.703 & Bituminous & Small & Ma jia zi \\
\hline 53 & Liaoning Province & Benxi City & NA & 41.245 & 124.342 & Anthracite & Small & Tian shi fu \\
\hline 54 & Liaoning Province & Benxi City & NA & 41.035 & 124.071 & Anthracite & Small & Cao he zhang \\
\hline 55 & Liaoning Province & $\begin{array}{l}\text { Huludao City } \\
\text { (formerly Jinxi City) }\end{array}$ & Jianchang County & 40.776 & 119.769 & Bituminous & Small & Bing gou \\
\hline 56 & Liaoning Province & Dandong City & Fengcheng City & 40.974 & 124.238 & Anthracite & Small & Dan dong, Li shu gou \\
\hline
\end{tabular}


Table 1. List of coal mines in China.-Continued

[Abbreviations used: dec. deg. - decimal degrees; NA - not available or not applicable]

\begin{tabular}{|c|c|c|c|c|c|c|c|c|}
\hline $\begin{array}{c}\text { ID } \\
\text { Number }\end{array}$ & $\begin{array}{c}\text { Province, National } \\
\text { Municipality, or } \\
\text { Autonomous Region }\end{array}$ & $\begin{array}{c}\text { Prefecture, Municipality, } \\
\text { Prefecture-Level City, or } \\
\text { League }\end{array}$ & $\begin{array}{l}\text { County, District or } \\
\text { County-Level City }\end{array}$ & $\begin{array}{l}\text { Latitude } \\
\text { (dec. deg.) }\end{array}$ & $\begin{array}{l}\text { Longitude } \\
\text { (dec. deg.) }\end{array}$ & Rank & $\begin{array}{l}\text { Relative } \\
\text { Mine Size }\end{array}$ & Mine Name \\
\hline 57 & Liaoning Province & Dandong City & Fengcheng City & 40.956 & 124.394 & Bituminous & Small & Dan dong, Gu jia \\
\hline 58 & Liaoning Province & Dandong City & Fengcheng City & 40.905 & 124.387 & Anthracite & Small & Feng cheng \\
\hline 1 & $\begin{array}{l}\text { Ningxia Hui } \\
\text { Autonomous Region }\end{array}$ & Shizuishang City & Pingluo County & 39.017 & 106.124 & Anthracite & Medium & Ru ji gou \\
\hline 2 & $\begin{array}{l}\text { Ningxia Hui } \\
\text { Autonomous Region }\end{array}$ & Shizuishang City & NA & 39.232 & 106.326 & Bituminous & Small & Shi tan jing \\
\hline 3 & $\begin{array}{l}\text { Ningxia Hui } \\
\text { Autonomous Region }\end{array}$ & Shizuishang City & NA & 39.250 & 106.379 & Bituminous & Small & Li jia gou \\
\hline 4 & $\begin{array}{l}\text { Ningxia Hui } \\
\text { Autonomous Region }\end{array}$ & Shizuishang City & NA & 39.128 & 106.387 & Anthracite & Small & Ma lian tan \\
\hline 5 & $\begin{array}{l}\text { Ningxia Hui } \\
\text { Autonomous Region }\end{array}$ & Shizuishang City & NA & 39.291 & 106.575 & Bituminous & Small & Sha ba tai \\
\hline 6 & $\begin{array}{l}\text { Ningxia Hui } \\
\text { Autonomous Region }\end{array}$ & Shizuishang City & NA & 39.202 & 106.601 & Bituminous & Medium & Zheng yi guan \\
\hline 7 & $\begin{array}{l}\text { Ningxia Hui } \\
\text { Autonomous Region }\end{array}$ & Shizuishang City & NA & 39.249 & 106.775 & Bituminous & Medium & \\
\hline 8 & $\begin{array}{l}\text { Ningxia Hui } \\
\text { Autonomous Region }\end{array}$ & Yinchuan City & Helan County & 38.765 & 105.928 & Anthracite & Small & Su yu kou \\
\hline 9 & $\begin{array}{l}\text { Ningxia Hui } \\
\text { Autonomous Region }\end{array}$ & Yinchuan City & Lingwu City & 38.188 & 106.485 & Bituminous & Large & $\begin{array}{l}\text { Heng shan bao } \\
\text { Ren jia zhuang }\end{array}$ \\
\hline 10 & $\begin{array}{l}\text { Ningxia Hui } \\
\text { Autonomous Region }\end{array}$ & Yinchuan City & Lingwu City & 38.244 & 106.489 & Bituminous & Large & $\begin{array}{l}\text { Heng shan bao } \\
\text { Hong shi wan }\end{array}$ \\
\hline 11 & $\begin{array}{l}\text { Ningxia Hui } \\
\text { Autonomous Region }\end{array}$ & Yinchuan City & Lingwu City & 38.160 & 106.517 & Bituminous & Small & $\begin{array}{l}\text { Heng shan bao } \\
\text { Ding jia liang }\end{array}$ \\
\hline 12 & $\begin{array}{l}\text { Ningxia Hui } \\
\text { Autonomous Region }\end{array}$ & Yinchuan City & Lingwu City & 38.228 & 106.557 & Bituminous & Medium & $\begin{array}{l}\text { Heng shan bao } \\
\text { Ma lian tai }\end{array}$ \\
\hline 13 & $\begin{array}{l}\text { Ningxia Hui } \\
\quad \text { Autonomous Region }\end{array}$ & Yinchuan City & Lingwu City & 38.050 & 106.647 & Bituminous & Small & Ci yao bao \\
\hline 14 & $\begin{array}{l}\text { Ningxia Hui } \\
\text { Autonomous Region }\end{array}$ & Yinchuan City & Lingwu City & 38.045 & 106.711 & Bituminous & Large & Yuan yang hu \\
\hline 15 & $\begin{array}{l}\text { Ningxia Hui } \\
\text { Autonomous Region }\end{array}$ & Zhongwei City & NA & 37.273 & 104.912 & Anthracite & Small & Liang shui yuan \\
\hline 17 & $\begin{array}{l}\text { Ningxia Hui } \\
\text { Autonomous Region }\end{array}$ & Zhongwei City & NA & 37.423 & 105.035 & Bituminous & Small & Xia he yan \\
\hline 18 & $\begin{array}{l}\text { Ningxia Hui } \\
\text { Autonomous Region }\end{array}$ & Zhongwei City & NA & 37.647 & 105.306 & Anthracite & Small & Dan liang shan \\
\hline 19 & $\begin{array}{l}\text { Ningxia Hui } \\
\text { Autonomous Region }\end{array}$ & Wuzhong City & Tongxin County & 37.010 & 105.326 & Bituminous & Small & Ma huang gou \\
\hline
\end{tabular}


Table 1. List of coal mines in China.-Continued

[Abbreviations used: dec. deg. - decimal degrees; NA - not available or not applicable]

\begin{tabular}{|c|c|c|c|c|c|c|c|c|}
\hline $\begin{array}{c}\text { ID } \\
\text { Number }\end{array}$ & $\begin{array}{l}\text { Province, National } \\
\text { Municipality, or } \\
\text { Autonomous Region }\end{array}$ & $\begin{array}{c}\text { Prefecture, Municipality, } \\
\text { Prefecture-Level City, or } \\
\text { League }\end{array}$ & $\begin{array}{l}\text { County, District or } \\
\text { County-Level City }\end{array}$ & $\begin{array}{l}\text { Latitude } \\
\text { (dec. deg.) }\end{array}$ & $\begin{array}{l}\text { Longitude } \\
\text { (dec. deg.) }\end{array}$ & Rank & $\begin{array}{l}\text { Relative } \\
\text { Mine Size }\end{array}$ & Mine Name \\
\hline 20 & $\begin{array}{l}\text { Ningxia Hui } \\
\text { Autonomous Region }\end{array}$ & Wuzhong City & Tongxin County & 37.090 & 105.440 & Bituminous & Small & Xia liu shui \\
\hline 21 & $\begin{array}{l}\text { Ningxia Hui } \\
\quad \text { Autonomous Region }\end{array}$ & Zhongwei City & Zhongning County & 37.663 & 105.567 & Anthracite & Small & Xian gou shan \\
\hline 22 & $\begin{array}{l}\text { Ningxia Hui } \\
\text { Autonomous Region }\end{array}$ & Wuzhong City & Tongxin County & 37.258 & 105.893 & Bituminous & Small & Tu po \\
\hline 23 & $\begin{array}{l}\text { Ningxia Hui } \\
\text { Autonomous Region }\end{array}$ & Wuzhong City & Tongxin County & 37.212 & 105.901 & Bituminous & Small & Xian tuo shi \\
\hline 24 & $\begin{array}{l}\text { Ningxia Hui } \\
\text { Autonomous Region }\end{array}$ & Wuzhong City & Tongxin County & 37.282 & 106.406 & Bituminous & Large & $\begin{array}{l}\text { Wei zhou Hong } \\
\text { cheng shui }\end{array}$ \\
\hline 25 & $\begin{array}{l}\text { Ningxia Hui } \\
\text { Autonomous Region }\end{array}$ & Yinchuan City & Lingwu City & 37.736 & 106.467 & Bituminous & Small & Shi gou yi \\
\hline 26 & $\begin{array}{l}\text { Ningxia Hui } \\
\text { Autonomous Region }\end{array}$ & Wuzhong City & Tongxin County & 37.257 & 106.484 & Bituminous & Large & Wei zhou \\
\hline 27 & $\begin{array}{l}\text { Ningxia Hui } \\
\quad \text { Autonomous Region }\end{array}$ & Wuzhong City & Tongxin County & 37.395 & 106.525 & Bituminous & Large & $\begin{array}{l}\text { Wei zhou } \\
\text { Tai yang shan }\end{array}$ \\
\hline 28 & $\begin{array}{l}\text { Ningxia Hui } \\
\text { Autonomous Region }\end{array}$ & Yinchuan City & Lingwu City & 37.969 & 106.573 & Bituminous & Large & Sui shi jing Zao quan \\
\hline 29 & $\begin{array}{l}\text { Ningxia Hui } \\
\text { Autonomous Region }\end{array}$ & Yinchuan City & Lingwu City & 37.999 & 106.673 & Bituminous & Large & $\begin{array}{l}\text { Sui shi jing } \\
\text { Yang chang wan }\end{array}$ \\
\hline 30 & $\begin{array}{l}\text { Ningxia Hui } \\
\text { Autonomous Region }\end{array}$ & Yinchuan City & Lingwu City & 37.848 & 106.710 & Bituminous & Large & Zhou jia gou \\
\hline 31 & $\begin{array}{l}\text { Ningxia Hui } \\
\text { Autonomous Region }\end{array}$ & Yinchuan City & Lingwu City & 37.728 & 106.726 & Bituminous & Large & Ji ia jing \\
\hline 32 & $\begin{array}{l}\text { Ningxia Hui } \\
\text { Autonomous Region }\end{array}$ & Wuzhong City & Yanchi County & 37.599 & 106.726 & Bituminous & Large & Shen jia zhuang \\
\hline 33 & $\begin{array}{l}\text { Ningxia Hui } \\
\text { Autonomous Region }\end{array}$ & Yinchuan City & Lingwu City & 37.829 & 106.814 & Bituminous & Large & Ma jia tan \\
\hline 34 & $\begin{array}{l}\text { Ningxia Hui } \\
\quad \text { Autonomous Region }\end{array}$ & Wuzhong City & Yanchi County & 37.464 & 106.829 & Bituminous & Large & Jin jia qu \\
\hline 35 & $\begin{array}{l}\text { Ningxia Hui } \\
\text { Autonomous Region }\end{array}$ & Wuzhong City & Yanchi County & 37.664 & 106.866 & Bituminous & Large & Feng ji gou \\
\hline 36 & $\begin{array}{l}\text { Ningxia Hui } \\
\text { Autonomous Region }\end{array}$ & Wuzhong City & Tongxin County & 36.971 & 106.201 & Bituminous & Small & Yao shan \\
\hline 37 & $\begin{array}{l}\text { Ningxia Hui } \\
\text { Autonomous Region }\end{array}$ & Guyuan City & NA & 36.399 & 106.277 & Bituminous & Small & Tan shan \\
\hline
\end{tabular}


Table 1. List of coal mines in China.-Continued

[Abbreviations used: dec. deg. - decimal degrees; NA - not available or not applicable]

\begin{tabular}{|c|c|c|c|c|c|c|c|c|}
\hline $\begin{array}{c}\text { ID } \\
\text { Number }\end{array}$ & $\begin{array}{l}\text { Province, National } \\
\text { Municipality, or } \\
\text { Autonomous Region }\end{array}$ & $\begin{array}{c}\text { Prefecture, Municipality, } \\
\text { Prefecture-Level City, or } \\
\text { League }\end{array}$ & $\begin{array}{l}\text { County, District or } \\
\text { County-Level City }\end{array}$ & $\begin{array}{l}\text { Latitude } \\
\text { (dec. deg.) }\end{array}$ & $\begin{array}{l}\text { Longitude } \\
\text { (dec. deg.) }\end{array}$ & Rank & $\begin{array}{l}\text { Relative } \\
\text { Mine Size }\end{array}$ & Mine Name \\
\hline 38 & $\begin{array}{l}\text { Ningxia Hui } \\
\quad \text { Autonomous Region }\end{array}$ & Guyuan City & Pengyang County & 36.227 & 106.624 & Bituminous & Medium & Wang wa \\
\hline 1 & Qinghai Province & $\begin{array}{l}\text { Haixi Autonomous } \\
\text { Prefecture }\end{array}$ & $\begin{array}{l}\text { Dachaidan } \\
\text { Administrative } \\
\text { Committee, } \\
\text { Dachaidan Town }\end{array}$ & 38.429 & 94.305 & Bituminous & Small & Jie lu su \\
\hline 2 & Qinghai Province & $\begin{array}{l}\text { Haixi Autonomous } \\
\text { Prefecture }\end{array}$ & $\begin{array}{l}\text { Dachaidan } \\
\text { Administrative } \\
\text { Committee, } \\
\text { Dachaidan Town }\end{array}$ & 38.019 & 94.988 & Bituminous & Small & Yu ka \\
\hline 3 & Qinghai Province & $\begin{array}{l}\text { Haixi Autonomous } \\
\text { Prefecture }\end{array}$ & Tianjun County & 38.571 & 98.399 & Bituminous & Small & Wa hu si \\
\hline 4 & Qinghai Province & $\begin{array}{l}\text { Haixi Autonomous } \\
\text { Prefecture }\end{array}$ & Tianjun County & 38.159 & 99.040 & Bituminous & Small & Duo suo gong ma \\
\hline 5 & Qinghai Province & $\begin{array}{l}\text { Haixi Autonomous } \\
\text { Prefecture }\end{array}$ & Tianjun County & 38.125 & 99.037 & Bituminous & Large & Ju hu geng \\
\hline 6 & Qinghai Province & $\begin{array}{l}\text { Haixi Autonomous } \\
\text { Prefecture }\end{array}$ & Tianjun County & 38.151 & 99.217 & Bituminous & Small & Hu shan \\
\hline 7 & Qinghai Province & $\begin{array}{l}\text { Haixi Autonomous } \\
\text { Prefecture }\end{array}$ & Tianjun County & 38.067 & 99.680 & Bituminous & Large & Jiang cang \\
\hline 8 & Qinghai Province & $\begin{array}{l}\text { Haibei Autonomous } \\
\text { Prefecture }\end{array}$ & Qilian County & 38.255 & 99.874 & Bituminous & Small & Yu niu gou \\
\hline 9 & Qinghai Province & $\begin{array}{l}\text { Haibei Autonomous } \\
\text { Prefecture }\end{array}$ & Qilian County & 38.235 & 100.380 & Bituminous & Small & $\begin{array}{l}\text { Qing yang gou bei, } \\
\text { No. } 12 \text { dao gou }\end{array}$ \\
\hline 10 & Qinghai Province & $\begin{array}{l}\text { Haibei Autonomous } \\
\text { Prefecture }\end{array}$ & Qilian County & 38.167 & 100.471 & Bituminous & Small & Qing yang gou \\
\hline 11 & Qinghai Province & $\begin{array}{l}\text { Haibei Autonomous } \\
\text { Prefecture }\end{array}$ & Qilian County & 38.061 & 100.615 & Bituminous & Small & A li ke \\
\hline 12 & Qinghai Province & $\begin{array}{l}\text { Haixi Autonomous } \\
\text { Prefecture }\end{array}$ & $\begin{array}{l}\text { Dachaidan } \\
\text { Administrative } \\
\text { Committee, } \\
\text { Dachaidan Town }\end{array}$ & 37.784 & 95.529 & Bituminous & Small & Da tou yang \\
\hline 13 & Qinghai Province & $\begin{array}{l}\text { Haixi Autonomous } \\
\text { Prefecture }\end{array}$ & $\begin{array}{l}\text { Dachaidan } \\
\text { Administrative } \\
\text { Committee, } \\
\text { Dachaidan Town }\end{array}$ & 37.612 & 95.694 & Bituminous & Small & Lu cao shan \\
\hline
\end{tabular}


Table 1. List of coal mines in China.-Continued

[Abbreviations used: dec. deg. - decimal degrees; NA - not available or not applicable]

\begin{tabular}{|c|c|c|c|c|c|c|c|c|}
\hline $\begin{array}{c}\text { ID } \\
\text { Number }\end{array}$ & $\begin{array}{l}\text { Province, National } \\
\text { Municipality, or } \\
\text { Autonomous Region }\end{array}$ & $\begin{array}{l}\text { Prefecture, Municipality, } \\
\text { Prefecture-Level City, or } \\
\text { League }\end{array}$ & $\begin{array}{l}\text { County, District or } \\
\text { County-Level City }\end{array}$ & $\begin{array}{l}\text { Latitude } \\
\text { (dec. deg.) }\end{array}$ & $\begin{array}{l}\text { Longitude } \\
\text { (dec. deg.) }\end{array}$ & Rank & $\begin{array}{l}\text { Relative } \\
\text { Mine Size }\end{array}$ & Mine Name \\
\hline 14 & Qinghai Province & $\begin{array}{l}\text { Haixi Autonomous } \\
\text { Prefecture }\end{array}$ & $\begin{array}{l}\text { Dachaidan } \\
\text { Administrative } \\
\text { Committee, } \\
\text { Dachaidan Town }\end{array}$ & 37.533 & 95.905 & Bituminous & Small & $\mathrm{Xi}$ da $\tan$ \\
\hline 15 & Qinghai Province & $\begin{array}{l}\text { Haixi Autonomous } \\
\text { Prefecture }\end{array}$ & Dulan County & 37.495 & 96.041 & Bituminous & Small & Ai nan \\
\hline 16 & Qinghai Province & $\begin{array}{l}\text { Haixi Autonomous } \\
\text { Prefecture }\end{array}$ & $\begin{array}{l}\text { Dachaidan } \\
\text { Administrative } \\
\text { Committee, } \\
\text { Dachaidan Town }\end{array}$ & 37.396 & 96.121 & Bituminous & Small & Da mei gou \\
\hline 17 & Qinghai Province & $\begin{array}{l}\text { Haixi Autonomous } \\
\text { Prefecture }\end{array}$ & $\begin{array}{l}\text { Dachaidan } \\
\text { Administrative } \\
\text { Committee, } \\
\text { Dachaidan Town }\end{array}$ & 37.017 & 96.496 & Bituminous & Small & Shi hui gou \\
\hline 18 & Qinghai Province & $\begin{array}{l}\text { Haixi Autonomous } \\
\text { Prefecture }\end{array}$ & Wulan (Ulan) County & 36.945 & 97.742 & Bituminous & Small & Wang ga xiu \\
\hline 19 & Qinghai Province & $\begin{array}{l}\text { Haixi Autonomous } \\
\text { Prefecture }\end{array}$ & Wulan (Ulan) County & 36.842 & 97.942 & Bituminous & Small & Mao niu shan \\
\hline 20 & Qinghai Province & $\begin{array}{l}\text { Haibei Autonomous } \\
\text { Prefecture }\end{array}$ & $\begin{array}{l}\text { Gangca (Gangcha) } \\
\text { County }\end{array}$ & 37.792 & 100.336 & Bituminous & Small & Wai li ha da \\
\hline 21 & Qinghai Province & $\begin{array}{l}\text { Haibei Autonomous } \\
\text { Prefecture }\end{array}$ & Qilian County & 37.688 & 100.559 & Bituminous & Small & Hai de er \\
\hline 22 & Qinghai Province & $\begin{array}{l}\text { Haibei Autonomous } \\
\text { Prefecture }\end{array}$ & Gangca County & 37.595 & 100.536 & Bituminous & Small & Re shui \\
\hline 23 & Qinghai Province & $\begin{array}{l}\text { Haibei Autonomous } \\
\text { Prefecture }\end{array}$ & Qilian County & 37.696 & 100.701 & Bituminous & Small & Mo le \\
\hline 24 & Qinghai Province & $\begin{array}{l}\text { Haibei Autonomous } \\
\text { Prefecture }\end{array}$ & Qilian County & 37.712 & 101.101 & Bituminous & Small & Duo luo \\
\hline 25 & Qinghai Province & $\begin{array}{l}\text { Haibei Autonomous } \\
\text { Prefecture }\end{array}$ & Menyuan County & 37.413 & 101.415 & Bituminous & Small & Tie bian \\
\hline 26 & Qinghai Province & $\begin{array}{l}\text { Haibei Autonomous } \\
\text { Prefecture }\end{array}$ & Menyuan County & 37.569 & 101.912 & Bituminous & Small & Ning chang he \\
\hline 27 & Qinghai Province & $\begin{array}{l}\text { Haibei Autonomous } \\
\text { Prefecture }\end{array}$ & Menyuan County & 37.318 & 101.665 & Bituminous & Small & Gua la \\
\hline 28 & Qinghai Province & $\begin{array}{l}\text { Haibei Autonomous } \\
\text { Prefecture }\end{array}$ & Menyuan County & 37.346 & 101.965 & Bituminous & Small & Ke tu \\
\hline 29 & Qinghai Province & Xining City & Huangyuan County & 36.502 & 101.191 & Bituminous & Small & Da cha shi lang \\
\hline 30 & Qinghai Province & Xining City & $\begin{array}{l}\text { Datong Autonomous } \\
\text { County }\end{array}$ & 36.888 & 101.652 & Bituminous & Small & Da tong \\
\hline
\end{tabular}


Table 1. List of coal mines in China.-Continued

[Abbreviations used: dec. deg. - decimal degrees; NA - not available or not applicable]

\begin{tabular}{|c|c|c|c|c|c|c|c|c|}
\hline $\begin{array}{c}\text { ID } \\
\text { Number }\end{array}$ & $\begin{array}{l}\text { Province, National } \\
\text { Municipality, or } \\
\text { Autonomous Region }\end{array}$ & $\begin{array}{c}\text { Prefecture, Municipality, } \\
\text { Prefecture-Level City, or } \\
\text { League }\end{array}$ & $\begin{array}{l}\text { County, District or } \\
\text { County-Level City }\end{array}$ & $\begin{array}{l}\text { Latitude } \\
\text { (dec. deg.) }\end{array}$ & $\begin{array}{l}\text { Longitude } \\
\text { (dec. deg.) }\end{array}$ & Rank & $\begin{array}{l}\text { Relative } \\
\text { Mine Size }\end{array}$ & Mine Name \\
\hline 31 & Qinghai Province & Haidong City & $\begin{array}{l}\text { Huzhu Autonomous } \\
\text { county }\end{array}$ & 36.925 & 101.824 & Bituminous & Small & Shi wan \\
\hline 32 & Qinghai Province & Haidong City & $\begin{array}{l}\text { Huzhu Autonomous } \\
\text { county }\end{array}$ & 36.589 & 101.933 & Bituminous & Small & Xiao xia \\
\hline 33 & Qinghai Province & Haibei City & Menyuan County & 37.328 & 102.281 & Bituminous & Small & Wan gu \\
\hline 34 & Qinghai Province & Haidong City & Ledu District & 36.311 & 102.122 & Bituminous & Small & Dong gou \\
\hline 35 & Qinghai Province & $\begin{array}{l}\text { Haixi Autonomous } \\
\text { Prefecture }\end{array}$ & Ge'ermu City & 34.146 & 92.302 & Bituminous & Small & Kai xin ling \\
\hline 36 & Qinghai Province & $\begin{array}{l}\text { Haixi Autonomous } \\
\text { Prefecture }\end{array}$ & Ge'ermu City & 34.353 & 92.749 & Bituminous & Small & Wu li \\
\hline 37 & Qinghai Province & $\begin{array}{l}\text { Haixi Autonomous } \\
\text { Prefecture }\end{array}$ & Ge'ermu City & 35.940 & 95.196 & Anthracite & Small & Qian jin \\
\hline 38 & Qinghai Province & $\begin{array}{l}\text { Haixi Autonomous } \\
\text { Prefecture }\end{array}$ & Dulan County & 35.732 & 96.449 & Bituminous & Small & Ba bao shan \\
\hline 40 & Qinghai Province & $\begin{array}{l}\text { Haixi Autonomous } \\
\text { Prefecture }\end{array}$ & Dulan County & 35.671 & 98.242 & Bituminous & Small & Ta tuo \\
\hline 41 & Qinghai Province & $\begin{array}{l}\text { Haixi Autonomous } \\
\text { Prefecture }\end{array}$ & Dulan County & 35.592 & 99.019 & Lignite & Small & Hei shan \\
\hline 42 & Qinghai Province & $\begin{array}{l}\text { Golog (Guoluo) } \\
\text { Autonomous Prefecture }\end{array}$ & Madoi (Maduo) County & 35.275 & 98.944 & Bituminous & Small & Hon tu po \\
\hline 43 & Qinghai Province & $\begin{array}{l}\text { Hainan Autonomous } \\
\text { Prefecture }\end{array}$ & Xinghai County & 35.332 & 99.310 & Bituminous & Small & Ku hai \\
\hline 44 & Qinghai Province & $\begin{array}{l}\text { Golog (Guoluo) } \\
\text { Autonomous Prefecture }\end{array}$ & Maqen (Maqin) County & 34.404 & 100.268 & Anthracite & Small & Da wu \\
\hline 45 & Qinghai Province & $\begin{array}{l}\text { Golog (Guoluo) } \\
\quad \text { Autonomous Prefecture }\end{array}$ & Maqen (Maqin) County & 34.336 & 100.509 & Bituminous & Small & Ye ma tan \\
\hline 48 & Qinghai Province & $\begin{array}{l}\text { Yushu Autonomous } \\
\text { Prefecture }\end{array}$ & Zadoi (Zaduo) County & 32.988 & 95.520 & Anthracite & Small & Ba ma \\
\hline 51 & Qinghai Province & $\begin{array}{l}\text { Yushu Autonomous } \\
\text { Prefecture }\end{array}$ & $\begin{array}{l}\text { Nangqen (Nanqian) } \\
\text { County }\end{array}$ & 32.091 & 96.238 & Bituminous & Small & Zha ran ning \\
\hline 52 & Qinghai Province & $\begin{array}{l}\text { Yushu Autonomous } \\
\text { Prefecture }\end{array}$ & $\begin{array}{l}\text { Nangqen (Nanqian) } \\
\text { County }\end{array}$ & 32.212 & 96.761 & Anthracite & Small & Bai lan gang \\
\hline 55 & Qinghai Province & $\begin{array}{l}\text { Yushu Autonomous } \\
\text { Prefecture }\end{array}$ & $\begin{array}{l}\text { Nangqen (Nanqian) } \\
\text { County }\end{array}$ & 31.910 & 95.781 & Anthracite & Small & Re yong \\
\hline 1 & Shaanxi Province & Yulin City & Shenmu County & 39.159 & 110.323 & Bituminous & Large & $\begin{array}{l}\text { Huo ji tu Coal } \\
\text { Well Field }\end{array}$ \\
\hline 2 & Shaanxi Province & Yulin City & Shenmu County & 39.069 & 110.500 & Bituminous & Medium & $\begin{array}{l}\text { Yu jia liang Coal } \\
\text { Well Field }\end{array}$ \\
\hline
\end{tabular}


Table 1. List of coal mines in China.-Continued

[Abbreviations used: dec. deg. - decimal degrees; NA - not available or not applicable]

\begin{tabular}{|c|c|c|c|c|c|c|c|c|}
\hline $\begin{array}{c}\text { ID } \\
\text { Number }\end{array}$ & $\begin{array}{l}\text { Province, National } \\
\text { Municipality, or } \\
\text { Autonomous Region }\end{array}$ & $\begin{array}{c}\text { Prefecture, Municipality, } \\
\text { Prefecture-Level City, or } \\
\text { League }\end{array}$ & $\begin{array}{l}\text { County, District or } \\
\text { County-Level City }\end{array}$ & $\begin{array}{l}\text { Latitude } \\
\text { (dec. deg.) }\end{array}$ & $\begin{array}{l}\text { Longitude } \\
\text { (dec. deg.) }\end{array}$ & Rank & $\begin{array}{l}\text { Relative } \\
\text { Mine Size }\end{array}$ & Mine Name \\
\hline 3 & Shaanxi Province & Yulin City & Fugu County & 39.096 & 110.705 & Bituminous & Medium & $\begin{array}{l}\text { Sha gou pen } \\
\text { Coal Well Field }\end{array}$ \\
\hline 4 & Shaanxi Province & Yulin City & Shenmu County & 38.789 & 110.411 & Bituminous & Small & $\begin{array}{l}\text { Da bian yao } \\
\text { Coal Well Field }\end{array}$ \\
\hline 6 & Shaanxi Province & Yulin City & Hengshan County & 38.065 & 109.266 & Bituminous & Medium & $\begin{array}{l}\text { Bo luo } \\
\quad \text { Coal Well Field }\end{array}$ \\
\hline 7 & Shaanxi Province & Yulin City & NA & 38.443 & 109.730 & Bituminous & Medium & $\begin{array}{l}\text { Jin ji tan } \\
\quad \text { Coal Well Field }\end{array}$ \\
\hline 8 & Shaanxi Province & Yulin City & NA & 38.397 & 109.851 & Bituminous & Medium & $\begin{array}{l}\text { Chang le bao } \\
\text { Coal Well Field }\end{array}$ \\
\hline 9 & Shaanxi Province & Yulin City & Hengshan County & 37.887 & 109.373 & Bituminous & Large & Bo luo Han pen \\
\hline 10 & Shaanxi Province & Yulin City & Wubao (Wubu) County & 37.577 & 110.721 & Bituminous & Medium & \\
\hline 11 & Shaanxi Province & Yan'an City & NA & 36.936 & 109.533 & Bituminous & Small & $\begin{array}{l}\text { Guan tun } \\
\text { Coal Well Field }\end{array}$ \\
\hline 12 & Shaanxi Province & Yan'an City & Fu County & 36.084 & 109.494 & Bituminous & Small & $\begin{array}{l}\text { Deng jia gou } \\
\text { Dang jia zhuang } \\
\text { Coal Well Field }\end{array}$ \\
\hline 15 & Shaanxi Province & Xianyang City & Bin County & 35.106 & 108.194 & Bituminous & Small & $\begin{array}{l}\text { Bai zi gou } \\
\text { Coal Well Field }\end{array}$ \\
\hline 16 & Shaanxi Province & Xianyang City & Bin County & 35.034 & 108.175 & Bituminous & Small & $\begin{array}{l}\text { Bai zi gou Coal Mine, } \\
\text { No. } 4 \text { Well Field }\end{array}$ \\
\hline 19 & Shaanxi Province & Xianyang City & Chunhua County & 35.017 & 108.564 & Bituminous & Small & $\begin{array}{l}\text { Tan ke } \\
\text { Coal Well Field }\end{array}$ \\
\hline 20 & Shaanxi Province & Tongchuan City & $\begin{array}{l}\text { Yaozhou District } \\
\text { (formerly Yao } \\
\text { County) }\end{array}$ & 35.091 & 108.669 & Bituminous & Small & $\begin{array}{l}\text { Zhao jin } \\
\quad \text { Coal Well Field }\end{array}$ \\
\hline 21 & Shaanxi Province & Xianyang City & Chunhua County & 35.068 & 108.732 & Bituminous & Small & $\begin{array}{l}\text { Hei tian yu } \\
\text { Coal Well Field }\end{array}$ \\
\hline 22 & Shaanxi Province & Tongchuan City & $\begin{array}{l}\text { Yaozhou District } \\
\text { (formerly Yao } \\
\text { County) }\end{array}$ & 35.197 & 108.799 & Bituminous & Large & $\begin{array}{l}\text { Chen jia shan } \\
\text { Coal Well Field }\end{array}$ \\
\hline 23 & Shaanxi Province & Tongchuan City & NA & 35.255 & 108.816 & Bituminous & Large & $\begin{array}{l}\text { Jiao ping Mining } \\
\text { Area, Cui jia wan } \\
\text { Coal Well Field }\end{array}$ \\
\hline 24 & Shaanxi Province & Tongchuan City & NA & 35.330 & 108.862 & Bituminous & Large & $\begin{array}{l}\text { Jiao ping Mining } \\
\text { Area, Long wang } \\
\text { Yu hua } \\
\text { Coal Well Field }\end{array}$ \\
\hline 25 & Shaanxi Province & Yan'an City & Huangling County & 35.407 & 108.878 & Bituminous & Small & Jian zhuang \\
\hline
\end{tabular}


Table 1. List of coal mines in China.-Continued

[Abbreviations used: dec. deg. - decimal degrees; NA - not available or not applicable]

\begin{tabular}{|c|c|c|c|c|c|c|c|c|}
\hline $\begin{array}{l}\text { ID } \\
\text { Number }\end{array}$ & $\begin{array}{l}\text { Province, National } \\
\text { Municipality, or } \\
\text { Autonomous Region }\end{array}$ & $\begin{array}{l}\text { Prefecture, Municipality, } \\
\text { Prefecture-Level City, or } \\
\text { League }\end{array}$ & $\begin{array}{l}\text { County, District or } \\
\text { County-Level City }\end{array}$ & $\begin{array}{l}\text { Latitude } \\
\text { (dec. deg.) }\end{array}$ & $\begin{array}{l}\text { Longitude } \\
\text { (dec. deg.) }\end{array}$ & Rank & $\begin{array}{l}\text { Relative } \\
\text { Mine Size }\end{array}$ & Mine Name \\
\hline 26 & Shaanxi Province & Yan'an City & Huangling County & 35.579 & 108.970 & Bituminous & Small & $\begin{array}{l}\text { Dian tou Mining Area, } \\
\text { An jiao } \\
\text { Coal Well Field }\end{array}$ \\
\hline 27 & Shaanxi Province & Yan'an City & Huangling County & 35.599 & 108.979 & Bituminous & Small & $\begin{array}{l}\text { Dian tou Mining Area, } \\
\text { Yu yuan } \\
\text { Coal Well Field }\end{array}$ \\
\hline 30 & Shaanxi Province & Yan'an City & Huangling County & 35.659 & 109.052 & Bituminous & Small & $\begin{array}{l}\text { Hong shi ya } \\
\text { Coal Well Field }\end{array}$ \\
\hline 31 & Shaanxi Province & Yan'an City & Huangling County & 35.719 & 109.017 & Bituminous & Large & $\begin{array}{l}\text { Dian tou Mining Area, } \\
\text { Li zhang he } \\
\text { Coal Well Field }\end{array}$ \\
\hline 32 & Shaanxi Province & Yan'an City & Fu County & 35.738 & 109.107 & Bituminous & Large & $\begin{array}{l}\text { Zhang cun yi Si jia } \\
\text { pen Coal Well Field }\end{array}$ \\
\hline 33 & Shaanxi Province & Tongchuan City & NA & 35.091 & 109.129 & Bituminous & Medium & $\begin{array}{l}\text { Tong chuan Mining } \\
\text { Area, San li dong } \\
\text { Coal Well Field }\end{array}$ \\
\hline 34 & Shaanxi Province & Tongchuan City & NA & 35.071 & 109.130 & Bituminous & Small & $\begin{array}{l}\text { Tong chuan Mining } \\
\text { Area, Huang bao } \\
\text { Yue jin } \\
\text { Coal Well Field }\end{array}$ \\
\hline 38 & Shaanxi Province & Tongchuan City & NA & 35.056 & 109.196 & Bituminous & Medium & $\begin{array}{l}\text { Tong chuan Mining } \\
\text { Area, Wang shi an } \\
\text { Coal Well Field }\end{array}$ \\
\hline 39 & Shaanxi Province & Yan'an City & Huangling County & 35.661 & 109.249 & Bituminous & Medium & $\begin{array}{l}\text { Dian tou Mining Area, } \\
\text { Lun cun } \\
\text { Coal Well Field }\end{array}$ \\
\hline 40 & Shaanxi Province & Tongchuan City & NA & 35.127 & 109.287 & Bituminous & Medium & $\begin{array}{l}\text { Tong chuan Mining } \\
\text { Area, Jin hua shan } \\
\text { Coal Well Field }\end{array}$ \\
\hline 43 & Shaanxi Province & Tongchuan City & NA & 35.121 & 109.336 & Bituminous & Medium & $\begin{array}{l}\text { Tong chuan Mining } \\
\text { Area, Ya kou } \\
\text { Coal Well Field }\end{array}$ \\
\hline 44 & Shaanxi Province & Tongchuan City & NA & 35.140 & 109.405 & Bituminous & Medium & $\begin{array}{l}\text { Tong chuan Mining } \\
\text { Area, Gao lou he } \\
\text { Coal Well Field }\end{array}$ \\
\hline 45 & Shaanxi Province & Tongchuan City & NA & 35.109 & 109.379 & Bituminous & Small & $\begin{array}{l}\text { Tong chuan Mining } \\
\text { Area, Xu jia gou } \\
\text { Coal Well Field }\end{array}$ \\
\hline
\end{tabular}


Table 1. List of coal mines in China.-Continued

[Abbreviations used: dec. deg. - decimal degrees; NA - not available or not applicable]

\begin{tabular}{|c|c|c|c|c|c|c|c|c|}
\hline $\begin{array}{c}\text { ID } \\
\text { Number }\end{array}$ & $\begin{array}{l}\text { Province, National } \\
\text { Municipality, or } \\
\text { Autonomous Region }\end{array}$ & $\begin{array}{c}\text { Prefecture, Municipality, } \\
\text { Prefecture-Level City, or } \\
\text { League }\end{array}$ & $\begin{array}{l}\text { County, District or } \\
\text { County-Level City }\end{array}$ & $\begin{array}{l}\text { Latitude } \\
\text { (dec. deg.) }\end{array}$ & $\begin{array}{l}\text { Longitude } \\
\text { (dec. deg.) }\end{array}$ & Rank & $\begin{array}{l}\text { Relative } \\
\text { Mine Size }\end{array}$ & Mine Name \\
\hline 47 & Shaanxi Province & Tongchuan City & NA & 35.147 & 109.430 & Bituminous & Medium & $\begin{array}{c}\text { Tong chuan Mining } \\
\text { Area, Dong po } \\
\text { Coal Well Field }\end{array}$ \\
\hline 48 & Shaanxi Province & Weinan City & Pucheng County & 35.112 & 109.498 & Bituminous & Medium & $\begin{array}{l}\text { Chu jia he } \\
\text { Coal Well Field }\end{array}$ \\
\hline 49 & Shaanxi Province & Weinan City & Baishui County & 35.189 & 109.539 & Bituminous & Small & $\begin{array}{l}\text { Liang shui quan } \\
\text { Coal Well Field }\end{array}$ \\
\hline 50 & Shaanxi Province & Weinan City & Baishui County & 35.233 & 109.568 & Bituminous & Small & $\begin{array}{l}\text { Nan jing tou } \\
\text { Coal Well Field }\end{array}$ \\
\hline 51 & Shaanxi Province & Weinan City & Pucheng County & 35.151 & 109.542 & Bituminous & Medium & $\begin{array}{l}\text { Ma cun } \\
\text { Coal Well Field }\end{array}$ \\
\hline 52 & Shaanxi Province & Weinan City & Pucheng County & 35.108 & 109.598 & Bituminous & Medium & $\begin{array}{l}\text { Nan qiao } \\
\text { Coal Well Field }\end{array}$ \\
\hline 53 & Shaanxi Province & Weinan City & Baishui County & 35.153 & 109.654 & Bituminous & Medium & $\begin{array}{l}\text { Bai shui } \\
\text { Coal Well Field }\end{array}$ \\
\hline 55 & Shaanxi Province & Weinan City & Baishui County & 35.174 & 109.718 & Bituminous & Medium & $\mathrm{Xi} \mathrm{gu}$ \\
\hline 57 & Shaanxi Province & Weinan City & Chengcheng County & 35.153 & 109.809 & Bituminous & Small & $\begin{array}{l}\text { Yao tou } \\
\text { Coal Well Field }\end{array}$ \\
\hline 58 & Shaanxi Province & Weinan City & Chengcheng County & 35.148 & 109.869 & Bituminous & Medium & $\begin{array}{l}\text { Quan jia he } \\
\text { Coal Well Field }\end{array}$ \\
\hline 59 & Shaanxi Province & Weinan City & Chengcheng County & 35.175 & 109.884 & Bituminous & Medium & $\begin{array}{l}\text { No. } 2 \text { Mine, } \\
\text { Coal Well Field }\end{array}$ \\
\hline 60 & Shaanxi Province & Weinan City & Chengcheng County & 35.235 & 109.914 & Bituminous & Medium & $\begin{array}{l}\text { Dong jia he } \\
\text { Coal Well Field }\end{array}$ \\
\hline 61 & Shaanxi Province & Weinan City & Chengcheng County & 35.195 & 109.966 & Bituminous & Medium & $\begin{array}{l}\text { Wang cun } \\
\text { Coal Well Field }\end{array}$ \\
\hline 62 & Shaanxi Province & Weinan City & Heyang County & 35.140 & 110.152 & Bituminous & Medium & $\begin{array}{l}\text { Wen ge } \\
\text { Coal Well Field }\end{array}$ \\
\hline 63 & Shaanxi Province & Weinan City & Heyang County & 35.200 & 110.188 & Bituminous & Large & $\begin{array}{l}\text { An yang } \\
\text { Coal Well Field }\end{array}$ \\
\hline 64 & Shaanxi Province & Weinan City & Hancheng City & 35.485 & 110.371 & Bituminous & Medium & $\begin{array}{l}\text { Southern } \\
\text { Coal Well Field }\end{array}$ \\
\hline 65 & Shaanxi Province & Weinan City & Hancheng City & 35.523 & 110.397 & Bituminous & Medium & $\begin{array}{l}\text { Ma jia qu } \\
\text { Coal Well Field }\end{array}$ \\
\hline 66 & Shaanxi Province & Weinan City & Hancheng City & 35.713 & 110.467 & Bituminous & Large & $\begin{array}{l}\text { Sang shu ping } \\
\text { Coal Well Field }\end{array}$ \\
\hline 67 & Shaanxi Province & Weinan City & Hancheng City & 35.652 & 110.499 & Anthracite & Large & $\begin{array}{l}\text { Xi gao qu } \\
\text { Coal Well Field }\end{array}$ \\
\hline
\end{tabular}


Table 1. List of coal mines in China.-Continued

[Abbreviations used: dec. deg. - decimal degrees; NA - not available or not applicable]

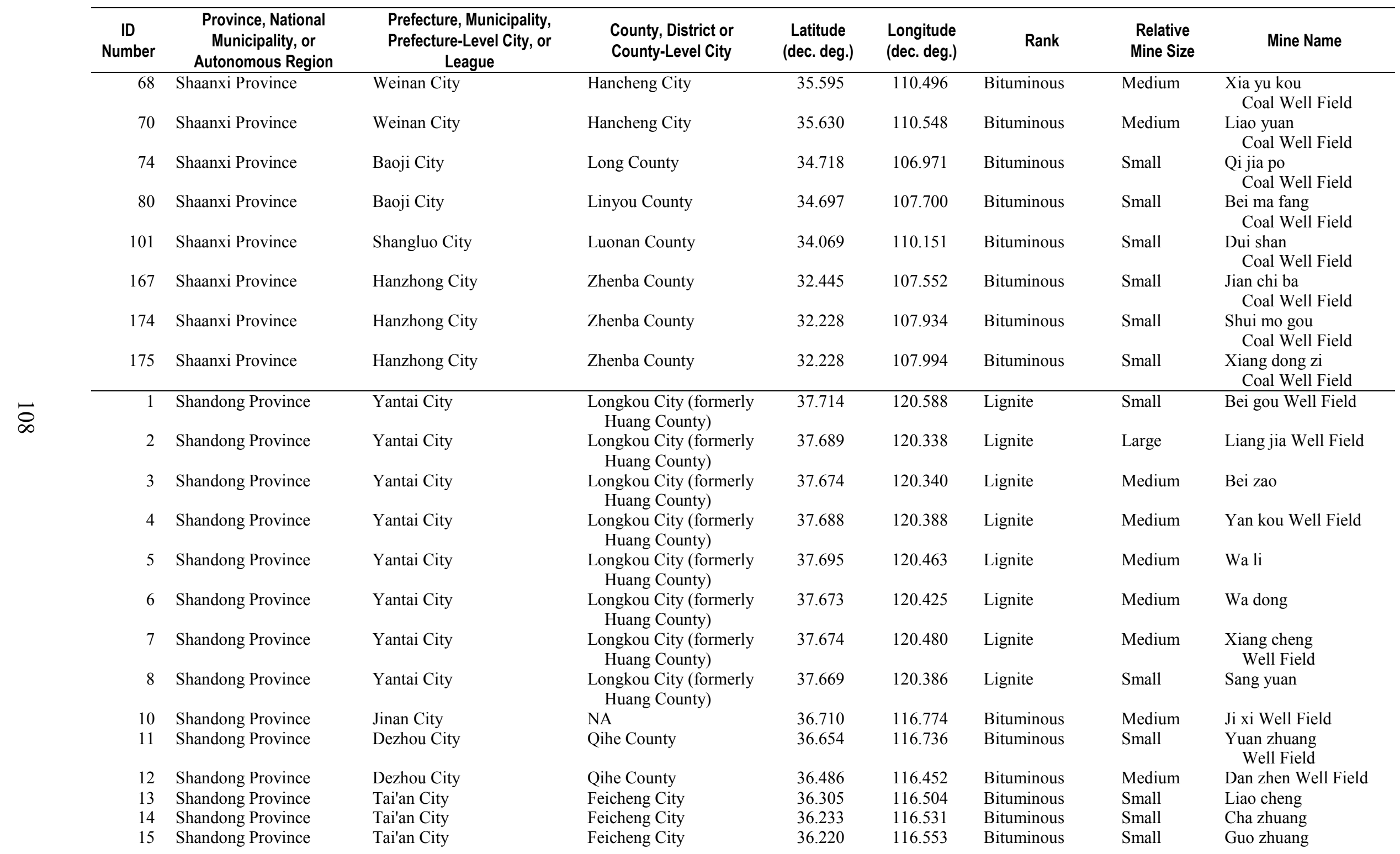


Table 1. List of coal mines in China. - Continued

[Abbreviations used: dec. deg. - decimal degrees; NA - not available or not applicable]

\begin{tabular}{|c|c|c|c|c|c|c|c|c|}
\hline $\begin{array}{c}\text { ID } \\
\text { Number }\end{array}$ & $\begin{array}{l}\text { Province, National } \\
\text { Municipality, or } \\
\text { Autonomous Region }\end{array}$ & $\begin{array}{c}\text { Prefecture, Municipality, } \\
\text { Prefecture-Level City, or } \\
\text { League }\end{array}$ & $\begin{array}{l}\text { County, District or } \\
\text { County-Level City }\end{array}$ & $\begin{array}{l}\text { Latitude } \\
\text { (dec. deg.) }\end{array}$ & $\begin{array}{l}\text { Longitude } \\
\text { (dec. deg.) }\end{array}$ & Rank & $\begin{array}{l}\text { Relative } \\
\text { Mine Size }\end{array}$ & Mine Name \\
\hline 16 & Shandong Province & Tai'an City & Feicheng City & 36.208 & 116.583 & Bituminous & Small & Nan gao yu \\
\hline 17 & Shandong Province & Tai'an City & Feicheng City & 36.200 & 116.528 & Bituminous & Small & Ping yin \\
\hline 18 & Shandong Province & Tai'an City & Feicheng City & 36.244 & 116.612 & Bituminous & Small & Bai zhuang \\
\hline 19 & Shandong Province & Tai'an City & Feicheng City & 36.227 & 116.643 & Bituminous & Small & Tao yang \\
\hline 20 & Shandong Province & Tai'an City & Feicheng City & 36.247 & 116.672 & Bituminous & Small & Da feng \\
\hline 21 & Shandong Province & Tai'an City & Feicheng City & 36.244 & 116.709 & Bituminous & Small & Yang zhuang \\
\hline 22 & Shandong Province & Tai'an City & Feicheng City & 36.268 & 116.736 & Bituminous & Small & Cao zhuang \\
\hline 23 & Shandong Province & Tai'an City & Feicheng City & 36.247 & 116.765 & Bituminous & Small & Wu li gou \\
\hline 25 & Shandong Province & Jinan City & Zhangqiu City & 36.697 & 117.351 & Anthracite & Small & $\mathrm{Xi} \mathrm{lu}$ \\
\hline 26 & Shandong Province & Jinan City & Zhangqiu City & 36.757 & 117.491 & Bituminous & Small & Lang gou \\
\hline 27 & Shandong Province & Jinan City & Zhangqiu City & 36.726 & 117.479 & Bituminous & Small & Bao zhuang \\
\hline 28 & Shandong Province & Jinan City & Zhangqiu City & 36.719 & 117.562 & Anthracite & Small & Dong feng \\
\hline 29 & Shandong Province & Jinan City & Zhangqiu City & 36.615 & 117.480 & Bituminous & Medium & Bu cun \\
\hline 30 & Shandong Province & Zibo City & NA & 36.634 & 117.720 & Bituminous & Small & Zou ping \\
\hline 31 & Shandong Province & Zibo City & NA & 36.642 & 117.743 & Bituminous & Small & Ling zi \\
\hline 32 & Shandong Province & Zibo City & NA & 36.633 & 117.804 & Bituminous & Small & Chong shan \\
\hline 33 & Shandong Province & Zibo City & NA & 36.633 & 117.804 & Bituminous & Small & Ling zi xiang \\
\hline 34 & Shandong Province & Zibo City & NA & 36.609 & 117.873 & Bituminous & Medium & Xia zhuang \\
\hline 35 & Shandong Province & Zibo City & NA & 36.601 & 117.943 & Bituminous & Small & Shi gu \\
\hline 36 & Shandong Province & Zibo City & NA & 36.583 & 117.961 & Bituminous & Small & Long quan \\
\hline 37 & Shandong Province & Zibo City & NA & 36.550 & 117.934 & Bituminous & Medium & $\mathrm{Xi}$ he \\
\hline 38 & Shandong Province & Zibo City & NA & 36.511 & 117.835 & Bituminous & Small & Zhai li \\
\hline 39 & Shandong Province & Zibo City & NA & 36.465 & 117.918 & Bituminous & Small & Hei shan \\
\hline 40 & Shandong Province & Laiwu City & NA & 36.194 & 117.576 & Anthracite & Small & $\begin{array}{r}\text { Yang zhuang } \\
\text { Well Field }\end{array}$ \\
\hline 41 & Shandong Province & Laiwu City & NA & 36.191 & 117.665 & Bituminous & Medium & E zhuang \\
\hline 42 & Shandong Province & Laiwu City & NA & 36.191 & 117.665 & Bituminous & Small & Nan ye \\
\hline 43 & Shandong Province & Laiwu City & NA & 36.191 & 117.665 & Anthracite & Small & Bei bu \\
\hline 44 & Shandong Province & Laiwu City & NA & 36.156 & 117.694 & Bituminous & Small & Zhai li \\
\hline 45 & Shandong Province & Laiwu City & NA & 36.138 & 117.712 & Bituminous & Small & $\mathrm{Xi}$ gang \\
\hline 46 & Shandong Province & Laiwu City & NA & 36.125 & 117.736 & Bituminous & Medium & Pan xi \\
\hline 47 & Shandong Province & Laiwu City & NA & 36.125 & 117.736 & Bituminous & Medium & Xin zhuang \\
\hline 48 & Shandong Province & Zibo City & Yiyuan County & 36.066 & 117.851 & Anthracite & Small & Huang zhuang \\
\hline 49 & Shandong Province & Zibo City & Yiyuan County & 36.171 & 117.975 & Bituminous & Small & Cao bu \\
\hline 50 & Shandong Province & Zibo City & NA & 36.824 & 118.255 & Bituminous & Small & Wang zhuang \\
\hline 51 & Shandong Province & Zibo City & NA & 36.784 & 118.111 & Anthracite & Small & Hu tian Sheng jian \\
\hline 52 & Shandong Province & Zibo City & NA & 36.755 & 118.037 & Anthracite & Small & Nan ding \\
\hline 53 & Shandong Province & Zibo City & NA & 36.747 & 118.078 & Bituminous & Small & Feng shui \\
\hline 54 & Shandong Province & Zibo City & NA & 36.722 & 118.008 & Bituminous & Small & Shuang gou \\
\hline
\end{tabular}


Table 1. List of coal mines in China. - Continued

[Abbreviations used: dec. deg. - decimal degrees; NA - not available or not applicable]

\begin{tabular}{|c|c|c|c|c|c|c|c|c|}
\hline $\begin{array}{c}\text { ID } \\
\text { Number }\end{array}$ & $\begin{array}{c}\text { Province, National } \\
\text { Municipality, or } \\
\text { Autonomous Region }\end{array}$ & $\begin{array}{c}\text { Prefecture, Municipality, } \\
\text { Prefecture-Level City, or } \\
\text { League }\end{array}$ & $\begin{array}{l}\text { County, District or } \\
\text { County-Level City }\end{array}$ & $\begin{array}{l}\text { Latitude } \\
\text { (dec. deg.) }\end{array}$ & $\begin{array}{l}\text { Longitude } \\
\text { (dec. deg.) }\end{array}$ & Rank & $\begin{array}{l}\text { Relative } \\
\text { Mine Size }\end{array}$ & Mine Name \\
\hline 55 & Shandong Province & Zibo City & NA & 36.687 & 118.045 & Bituminous & Small & Hong shan \\
\hline 56 & Shandong Province & Weifang City & Changle County & 36.746 & 118.924 & Bituminous & Small & Zhu liu dian \\
\hline 57 & Shandong Province & Weifang City & Changle County & 36.631 & 118.913 & Lignite & Small & Wu tu \\
\hline 58 & Shandong Province & Weifang City & Linqu County & 36.494 & 118.435 & Bituminous & Small & Yu jing \\
\hline 59 & Shandong Province & Zibo City & Yiyuan County & 36.188 & 118.035 & Bituminous & Small & Lu cun \\
\hline 60 & Shandong Province & Weifang City & NA & 36.590 & 119.143 & Anthracite & Medium & Fang zi \\
\hline 62 & Shandong Province & Heze City & Yuncheng County & 35.474 & 115.943 & Bituminous & Large & \\
\hline 63 & Shandong Province & Jining City & Liangshan County & 35.988 & 116.081 & Anthracite & Large & Dai miao \\
\hline 64 & Shandong Province & Tai'an City & Ningyang County & 35.807 & 116.837 & Bituminous & Medium & \\
\hline 65 & Shandong Province & Jining City & Qufu City & 35.597 & 116.834 & Bituminous & Large & Jing tian \\
\hline 66 & Shandong Province & Jining City & NA & 35.448 & 116.562 & Bituminous & Large & Dai zhuang Well Field \\
\hline 67 & Shandong Province & Jining City & NA & 35.449 & 116.659 & Bituminous & Large & $\mathrm{Xu}$ guang Well Field \\
\hline 68 & Shandong Province & Jining City & Yanzhou City & 35.490 & 116.857 & Bituminous & Large & Xing long zhuang \\
\hline 69 & Shandong Province & Jining City & Yanzhou City & 35.453 & 116.793 & Bituminous & Small & $\begin{array}{l}\text { Yang zhuang } \\
\text { Well Field }\end{array}$ \\
\hline 70 & Shandong Province & Jining City & Yanzhou City & 35.439 & 116.764 & Bituminous & Small & $\begin{array}{l}\text { Tian zhuang } \\
\text { Well Field }\end{array}$ \\
\hline 71 & Shandong Province & Jining City & Yanzhou City & 35.416 & 116.737 & Bituminous & Small & Xin ji Well Field \\
\hline 72 & Shandong Province & Jining City & Zoucheng City & 35.435 & 116.883 & Bituminous & Large & Dong tan Well Field \\
\hline 73 & Shandong Province & Jining City & Zoucheng City & 35.381 & 116.870 & Bituminous & Large & Nan tun \\
\hline 74 & Shandong Province & Jining City & Zoucheng City & 35.362 & 116.781 & Bituminous & Medium & Li yan Well Field \\
\hline 75 & Shandong Province & Jining City & Zoucheng City & 35.351 & 116.889 & Bituminous & Small & Bei su \\
\hline 76 & Shandong Province & Jining City & Zoucheng City & 35.328 & 116.838 & Bituminous & Small & Luo ling \\
\hline 77 & Shandong Province & Jining City & Zoucheng City & 35.340 & 116.936 & Bituminous & Small & Tang cun \\
\hline 78 & Shandong Province & Jining City & NA & 35.361 & 116.596 & Bituminous & Large & No. 2 Well Field \\
\hline 79 & Shandong Province & Jining City & NA & 35.268 & 116.623 & Bituminous & Large & No. 3 Well Field \\
\hline 80 & Shandong Province & Jining City & Weishan County & 35.242 & 116.688 & Bituminous & Medium & Si he ya Well Field \\
\hline 81 & Shandong Province & Jining City & Zoucheng City & 35.284 & 116.843 & Bituminous & Large & Bao dian \\
\hline 82 & Shandong Province & Zaozhuang City & Tengzhou City & 35.102 & 116.897 & Bituminous & Medium & Bei xu lou Well Field \\
\hline 83 & Shandong Province & Zaozhuang City & Tengzhou City & 35.118 & 116.945 & Bituminous & Small & Liu zhuang Well Field \\
\hline 84 & Shandong Province & Zaozhuang City & Tengzhou City & 35.094 & 116.991 & Bituminous & Medium & Xiu cheng Well Field \\
\hline 85 & Shandong Province & Zaozhuang City & Tengzhou City & 35.060 & 116.927 & Bituminous & Small & Ji suo Well Field \\
\hline 86 & Shandong Province & Zaozhuang City & Tengzhou City & 35.049 & 116.946 & Bituminous & Medium & Wang chao Well Field \\
\hline 87 & Shandong Province & Tai'an City & Ningyang County & 35.865 & 117.149 & Bituminous & Medium & Hua feng \\
\hline 88 & Shandong Province & Tai'an City & Xintai City & 35.900 & 117.302 & Bituminous & Small & Yu cun \\
\hline 89 & Shandong Province & Tai'an City & Xintai City & 35.967 & 117.617 & Bituminous & Medium & Quan gou \\
\hline 90 & Shandong Province & Tai'an City & Xintai City & 35.896 & 117.642 & Bituminous & Small & Wang jia zhai \\
\hline 91 & Shandong Province & Tai'an City & Xintai City & 35.893 & 117.607 & Bituminous & Medium & Xie zhuang \\
\hline 92 & Shandong Province & Tai'an City & Xintai City & 35.883 & 117.644 & Bituminous & Medium & Sun cun \\
\hline
\end{tabular}


Table 1. List of coal mines in China. - Continued

[Abbreviations used: dec. deg. - decimal degrees; NA - not available or not applicable]

\begin{tabular}{|c|c|c|c|c|c|c|c|c|}
\hline $\begin{array}{c}\text { ID } \\
\text { Number }\end{array}$ & $\begin{array}{c}\text { Province, National } \\
\text { Municipality, or } \\
\text { Autonomous Region }\end{array}$ & $\begin{array}{c}\text { Prefecture, Municipality, } \\
\text { Prefecture-Level City, or } \\
\text { League }\end{array}$ & $\begin{array}{l}\text { County, District or } \\
\text { County-Level City }\end{array}$ & $\begin{array}{l}\text { Latitude } \\
\text { (dec. deg.) }\end{array}$ & $\begin{array}{l}\text { Longitude } \\
\text { (dec. deg.) }\end{array}$ & Rank & $\begin{array}{l}\text { Relative } \\
\text { Mine Size }\end{array}$ & Mine Name \\
\hline 93 & Shandong Province & Tai'an City & Xintai City & 35.884 & 117.645 & Bituminous & Medium & Di zhen \\
\hline 94 & Shandong Province & Tai'an City & Xintai City & 35.884 & 117.645 & Bituminous & Medium & $\begin{array}{l}\text { Quan gou No. } 2 \\
\text { Well Field }\end{array}$ \\
\hline 95 & Shandong Province & Tai'an City & Xintai City & 35.884 & 117.645 & Bituminous & Medium & Liang zhuang \\
\hline 96 & Shandong Province & Tai'an City & Xintai City & 35.884 & 117.645 & Bituminous & Medium & Wen he \\
\hline 97 & Shandong Province & Tai'an City & Xintai City & 35.866 & 117.700 & Bituminous & Medium & Wen nan \\
\hline 98 & Shandong Province & Tai'an City & Xintai City & 35.824 & 117.752 & Bituminous & Medium & Zhang zhuang \\
\hline 99 & Shandong Province & Tai'an City & Xintai City & 35.798 & 117.781 & Bituminous & Small & Cao zhuang \\
\hline 100 & Shandong Province & Tai'an City & Xintai City & 35.798 & 117.781 & Bituminous & Small & $\begin{array}{l}\text { Wen nan No. } 57 \\
\text { Coal Mine }\end{array}$ \\
\hline 101 & Shandong Province & Tai'an City & Xintai City & 35.798 & 117.781 & Bituminous & Small & Shang zhuang \\
\hline 102 & Shandong Province & Linyi City & Mengyin County & 35.765 & 117.884 & Bituminous & Small & Hong gou \\
\hline 103 & Shandong Province & Linyi City & Pingyi County & 35.648 & 117.602 & Bituminous & Small & Qi shan \\
\hline 104 & Shandong Province & Zaozhuang City & Tengzhou City & 35.612 & 117.606 & Bituminous & Small & Zhong cun \\
\hline 105 & Shandong Province & Zaozhuang City & Tengzhou City & 35.113 & 117.034 & Bituminous & Medium & Xu zhuang Well Field \\
\hline 106 & Shandong Province & Zaozhuang City & Tengzhou City & 35.089 & 117.039 & Bituminous & Small & Wu suo tun Well Field \\
\hline 107 & Shandong Province & Zaozhuang City & Tengzhou City & 35.072 & 117.002 & Bituminous & Small & Zhao po \\
\hline 108 & Shandong Province & Zaozhuang City & Tengzhou City & 35.037 & 117.080 & Bituminous & Small & Quan shang Well Field \\
\hline 109 & Shandong Province & Rizhao City & Ju County & 35.467 & 118.682 & Bituminous & Small & \\
\hline 110 & Shandong Province & Linyi City & NA & 35.145 & 118.205 & Bituminous & Small & Zhu li \\
\hline 111 & Shandong Province & Jining City & Weishan County & 34.927 & 116.974 & Bituminous & Small & Cai yuan \\
\hline 112 & Shandong Province & Zaozhuang City & Tengzhou City & 34.956 & 117.025 & Bituminous & Large & Chai li \\
\hline 113 & Shandong Province & Zaozhuang City & Tengzhou City & 34.976 & 117.068 & Bituminous & Small & Guo zhuang \\
\hline 114 & Shandong Province & Jining City & Weishan County & 34.975 & 117.136 & Bituminous & Medium & Sheng jian \\
\hline 115 & Shandong Province & Zaozhuang City & Tengzhou City & 34.945 & 117.099 & Bituminous & Medium & Tian chen Well Field \\
\hline 116 & Shandong Province & Zaozhuang City & Tengzhou City & 34.960 & 117.265 & Bituminous & Small & Wei zhuang \\
\hline 117 & Shandong Province & Zaozhuang City & Tengzhou City & 34.923 & 117.248 & Bituminous & Medium & No. 81 Coal Mine \\
\hline 118 & Shandong Province & Jining City & Weishan County & 34.853 & 117.042 & Bituminous & Large & Jiang zhuang \\
\hline 119 & Shandong Province & Jining City & Weishan County & 34.860 & 117.064 & Bituminous & Large & Fu cun Well Field \\
\hline 120 & Shandong Province & Jining City & Weishan County & 34.840 & 117.122 & Bituminous & Large & Xu lou Well Field \\
\hline 121 & Shandong Province & Zaozhuang City & Tengzhou City & 34.882 & 117.232 & Bituminous & Small & Hou huang zhuang \\
\hline 122 & Shandong Province & Zaozhuang City & NA & 34.857 & 117.254 & Bituminous & Small & Hou wan Well Field \\
\hline 123 & Shandong Province & Zaozhuang City & Tengzhou City & 34.848 & 117.222 & Bituminous & Small & Guan qiao Well Field \\
\hline 124 & Shandong Province & Zaozhuang City & $\mathrm{NA}$ & 34.897 & 117.365 & Bituminous & Small & Shan jia lin \\
\hline 125 & Shandong Province & Zaozhuang City & NA & 34.899 & 117.497 & Bituminous & Small & Zhu zi bu \\
\hline 126 & Shandong Province & Zaozhuang City & NA & 34.864 & 117.414 & Bituminous & Small & Gan lin \\
\hline 127 & Shandong Province & Zaozhuang City & NA & 34.846 & 117.336 & Bituminous & Medium & Tao zhuang \\
\hline 128 & Shandong Province & Zaozhuang City & NA & 34.858 & 117.628 & Bituminous & Medium & Zao zhuang \\
\hline 129 & Shandong Province & Zaozhuang City & NA & 34.816 & 117.477 & Bituminous & Small & Zhai zi \\
\hline
\end{tabular}


Table 1. List of coal mines in China. - Continued

[Abbreviations used: dec. deg. - decimal degrees; NA - not available or not applicable]

\begin{tabular}{|c|c|c|c|c|c|c|c|c|c|}
\hline & $\begin{array}{c}\text { ID } \\
\text { Number }\end{array}$ & $\begin{array}{c}\text { Province, National } \\
\text { Municipality, or } \\
\text { Autonomous Region }\end{array}$ & $\begin{array}{c}\text { Prefecture, Municipality, } \\
\text { Prefecture-Level City, or } \\
\text { League }\end{array}$ & $\begin{array}{l}\text { County, District or } \\
\text { County-Level City }\end{array}$ & $\begin{array}{l}\text { Latitude } \\
\text { (dec. deg.) }\end{array}$ & $\begin{array}{l}\text { Longitude } \\
\text { (dec. deg.) }\end{array}$ & Rank & $\begin{array}{l}\text { Relative } \\
\text { Mine Size }\end{array}$ & Mine Name \\
\hline & 130 & Shandong Province & Zaozhuang City & NA & 34.794 & 117.534 & Bituminous & Small & Huang zhuang \\
\hline & 131 & Shandong Province & Zaozhuang City & NA & 34.812 & 117.583 & Bituminous & Small & Guan zhuang \\
\hline & 132 & Shandong Province & Zaozhuang City & NA & 34.562 & 117.397 & Bituminous & Small & Zhang shan zi \\
\hline & 133 & Shandong Province & Linyi City & NA & 34.926 & 118.006 & Bituminous & Small & Zhu chen \\
\hline & 134 & Shandong Province & Linyi City & NA & 35.001 & 118.374 & Anthracite & Small & Ge zhuang \\
\hline & 135 & Shandong Province & Linyi City & NA & 34.986 & 118.291 & Bituminous & Small & Luo zhuang \\
\hline & 136 & Shandong Province & Linyi City & NA & 34.971 & 118.371 & Bituminous & Small & Dong gao du \\
\hline & 137 & Shandong Province & Linyi City & NA & 34.954 & 118.232 & Bituminous & Small & $\mathrm{Xi}$ shan \\
\hline & 138 & Shandong Province & Linyi City & NA & 34.954 & 118.232 & Bituminous & Small & Huang tu nian \\
\hline & 139 & Shandong Province & Linyi City & NA & 34.951 & 118.285 & Anthracite & Small & Quan zhuang \\
\hline & 140 & Shandong Province & Linyi City & NA & 34.949 & 118.374 & Bituminous & Small & Xi gao du \\
\hline & 141 & Shandong Province & Linyi City & NA & 34.949 & 118.374 & Bituminous & Small & Xiao zhuang zi \\
\hline & 142 & Shandong Province & Linyi City & NA & 34.949 & 118.374 & Bituminous & Small & Tang ya \\
\hline & 143 & Shandong Province & Linyi City & NA & 34.933 & 118.221 & Anthracite & Small & Ce shan \\
\hline & 144 & Shandong Province & Linyi City & NA & 34.910 & 118.252 & Bituminous & Small & Tang zhuang \\
\hline & 145 & Shandong Province & Linyi City & NA & 34.912 & 118.250 & Bituminous & Small & Lao tun \\
\hline - & 146 & Shandong Province & Linyi City & NA & 34.926 & 118.293 & Bituminous & Small & Da lu hu \\
\hline 戸 & 147 & Shandong Province & Linyi City & NA & 34.917 & 118.319 & Anthracite & Small & Wu si zhuang \\
\hline & 148 & Shandong Province & Linyi City & NA & 34.869 & 118.261 & Anthracite & Small & Ju zhuang \\
\hline & 149 & Shandong Province & Linyi City & Tancheng County & 34.874 & 118.352 & Bituminous & Small & Hei hu dun \\
\hline & 150 & Shandong Province & Linyi City & Tancheng County & 34.822 & 118.187 & Bituminous & Small & Chu dun \\
\hline & 151 & Shandong Province & Linyi City & Tancheng County & 34.835 & 118.367 & Bituminous & Small & Wu he \\
\hline & 152 & Shandong Province & Linyi City & Tancheng County & 34.820 & 118.363 & Bituminous & Small & Zhu bai \\
\hline & 1 & Shanxi Province & Datong City & Zuoyun County & 40.092 & 112.823 & Bituminous & Large & Dong zhou yao qu \\
\hline & 2 & Shanxi Province & Datong City & Zuoyun County & 40.041 & 112.920 & Bituminous & Large & Ma kou mu dai \\
\hline & 3 & Shanxi Province & Datong City & $\mathrm{NA}$ & 40.041 & 112.920 & Bituminous & Large & Ma ji liang Well Field \\
\hline & 4 & Shanxi Province & Datong City & NA & 40.041 & 112.920 & Bituminous & Large & Yan zi shan Well Field \\
\hline & 5 & Shanxi Province & Datong City & NA & 40.041 & 112.920 & Bituminous & Large & $\begin{array}{l}\text { North Carboniferous } \\
\text { Period Detailed } \\
\text { Survey Area }\end{array}$ \\
\hline & 6 & Shanxi Province & Datong City & NA & 40.115 & 112.981 & Bituminous & Large & Si tai gou Well Field \\
\hline & 7 & Shanxi Province & Datong City & NA & 40.140 & 113.126 & Bituminous & Large & Jiang jia wan \\
\hline & 8 & Shanxi Province & Datong City & NA & 40.140 & 113.126 & Bituminous & Large & Yun gang Well Field \\
\hline & 9 & Shanxi Province & Datong City & NA & 40.140 & 113.126 & Bituminous & Large & Wu guan tun \\
\hline & 10 & Shanxi Province & Datong City & NA & 40.140 & 113.126 & Bituminous & Large & Qing ci yao \\
\hline & 11 & Shanxi Province & Datong City & NA & 40.140 & 113.126 & Bituminous & Large & $\begin{array}{l}\text { Jin hua gong } \\
\text { Well Field }\end{array}$ \\
\hline & 12 & Shanxi Province & Datong City & NA & 40.140 & 113.126 & Bituminous & Large & Nan shan Well \\
\hline & 13 & Shanxi Province & Datong City & NA & 40.040 & 113.089 & Bituminous & Large & Da dou gou Well Field \\
\hline
\end{tabular}


Table 1. List of coal mines in China.-Continued

[Abbreviations used: dec. deg. - decimal degrees; NA - not available or not applicable]

\begin{tabular}{|c|c|c|c|c|c|c|c|c|}
\hline $\begin{array}{c}\text { ID } \\
\text { Number }\end{array}$ & $\begin{array}{l}\text { Province, National } \\
\text { Municipality, or } \\
\text { Autonomous Region }\end{array}$ & $\begin{array}{c}\text { Prefecture, Municipality, } \\
\text { Prefecture-Level City, or } \\
\text { League }\end{array}$ & $\begin{array}{l}\text { County, District or } \\
\text { County-Level City }\end{array}$ & $\begin{array}{l}\text { Latitude } \\
\text { (dec. deg.) }\end{array}$ & $\begin{array}{l}\text { Longitude } \\
\text { (dec. deg.) }\end{array}$ & Rank & $\begin{array}{l}\text { Relative } \\
\text { Mine Size }\end{array}$ & Mine Name \\
\hline 14 & Shanxi Province & Datong City & NA & 40.040 & 113.089 & Bituminous & Large & Bai dong Well Field \\
\hline 15 & Shanxi Province & Datong City & NA & 40.040 & 113.089 & Bituminous & Large & $\begin{array}{l}\text { Yong ding zhuang } \\
\text { Well Field }\end{array}$ \\
\hline 16 & Shanxi Province & Datong City & NA & 40.040 & 113.089 & Bituminous & Large & Mei yu kou Well Field \\
\hline 17 & Shanxi Province & Datong City & NA & 40.040 & 113.089 & Bituminous & Large & $\begin{array}{l}\text { Tong jia liang } \\
\text { Well Field }\end{array}$ \\
\hline 18 & Shanxi Province & Datong City & NA & 40.106 & 113.304 & Bituminous & Large & Xin zhou yao Mine \\
\hline 19 & Shanxi Province & Datong City & Yanggao County & 40.322 & 113.595 & Bituminous & Small & Guo jia Well Field \\
\hline 20 & Shanxi Province & Xinzhou City & Baode County & 39.031 & 111.162 & Bituminous & Large & Jia jia Well Field \\
\hline 21 & Shanxi Province & Xinzhou City & Baode County & 39.031 & 111.162 & Bituminous & Large & $\begin{array}{l}\text { Dong guan Lu zi gou } \\
\text { Accurately } \\
\text { Surveyed Area }\end{array}$ \\
\hline 22 & Shanxi Province & Xinzhou City & Hequ County & 39.176 & 111.261 & Bituminous & Large & $\begin{array}{l}\text { Sha ping No. } 1 \\
\text { Open Pit } \\
\text { Exploration Area }\end{array}$ \\
\hline 23 & Shanxi Province & Xinzhou City & Hequ County & 39.176 & 111.261 & Bituminous & Large & $\begin{array}{l}\text { Sha ping } \\
\text { Exploration Area }\end{array}$ \\
\hline 24 & Shanxi Province & Xinzhou City & Pianguan County & 39.418 & 111.434 & Bituminous & Large & $\begin{array}{l}\text { Ci yao gou } \\
\text { Exploration Area }\end{array}$ \\
\hline 25 & Shanxi Province & Shuozhou City & NA & 39.510 & 112.277 & Bituminous & Large & $\begin{array}{l}\text { Ping lu Jing ping } \\
\text { Exploraration Area }\end{array}$ \\
\hline 26 & Shanxi Province & Shuozhou City & NA & 39.575 & 112.318 & Bituminous & Large & $\begin{array}{l}\text { Ping lu Ping fan cheng } \\
\text { Exploraration Area }\end{array}$ \\
\hline 27 & Shanxi Province & Shuozhou City & NA & 39.499 & 112.370 & Bituminous & Large & $\begin{array}{l}\text { Ping lu Cao zhuang } \\
\text { Exploraration Area }\end{array}$ \\
\hline 28 & Shanxi Province & Shuozhou City & NA & 39.499 & 112.370 & Bituminous & Large & $\begin{array}{l}\text { Ping lu An tai bao } \\
\text { Open Area }\end{array}$ \\
\hline 29 & Shanxi Province & Shuozhou City & NA & 39.538 & 112.522 & Bituminous & Large & $\begin{array}{l}\text { Ping lu Ma guan he } \\
\text { dong } \\
\text { Reconnaissance } \\
\text { Exploration Area }\end{array}$ \\
\hline 30 & Shanxi Province & Shuozhou City & NA & 39.441 & 112.427 & Bituminous & Large & $\begin{array}{l}\text { Ping lu An jia ling } \\
\text { Exploration Area }\end{array}$ \\
\hline 31 & Shanxi Province & Shuozhou City & NA & 39.441 & 112.427 & Bituminous & Large & $\begin{array}{l}\text { Liu jia kou Coal Mine, } \\
\text { East Well }\end{array}$ \\
\hline 32 & Shanxi Province & Shuozhou City & NA & 39.441 & 112.427 & Bituminous & Large & $\begin{array}{l}\text { Zhi yu } \\
\text { Open Mining Area }\end{array}$ \\
\hline 33 & Shanxi Province & Shuozhou City & NA & 39.441 & 112.427 & Bituminous & Large & Yang dong Coal Mine \\
\hline
\end{tabular}


Table 1. List of coal mines in China.-Continued

[Abbreviations used: dec. deg. - decimal degrees; NA - not available or not applicable]

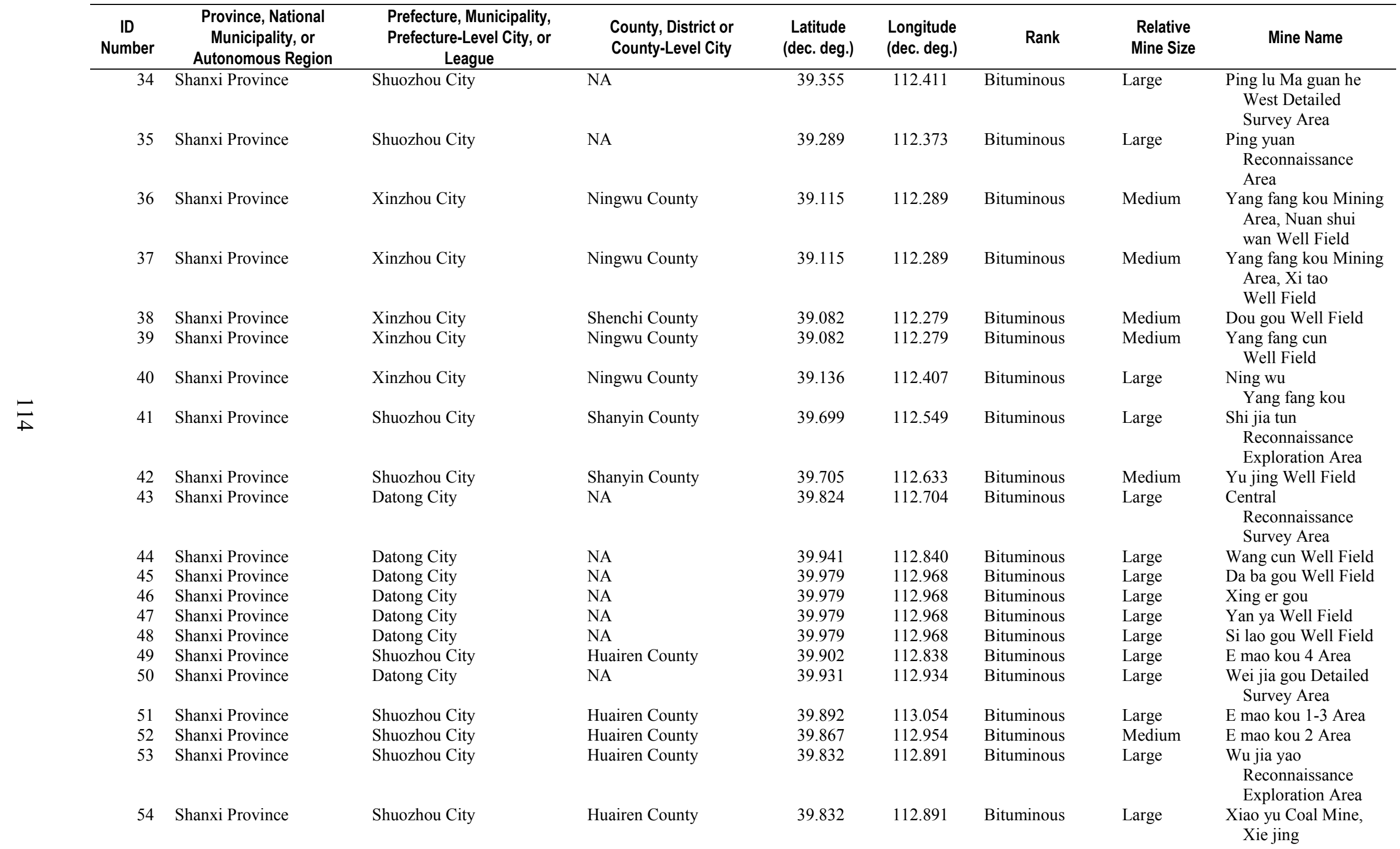


Table 1. List of coal mines in China. - Continued

[Abbreviations used: dec. deg. - decimal degrees; NA - not available or not applicable]

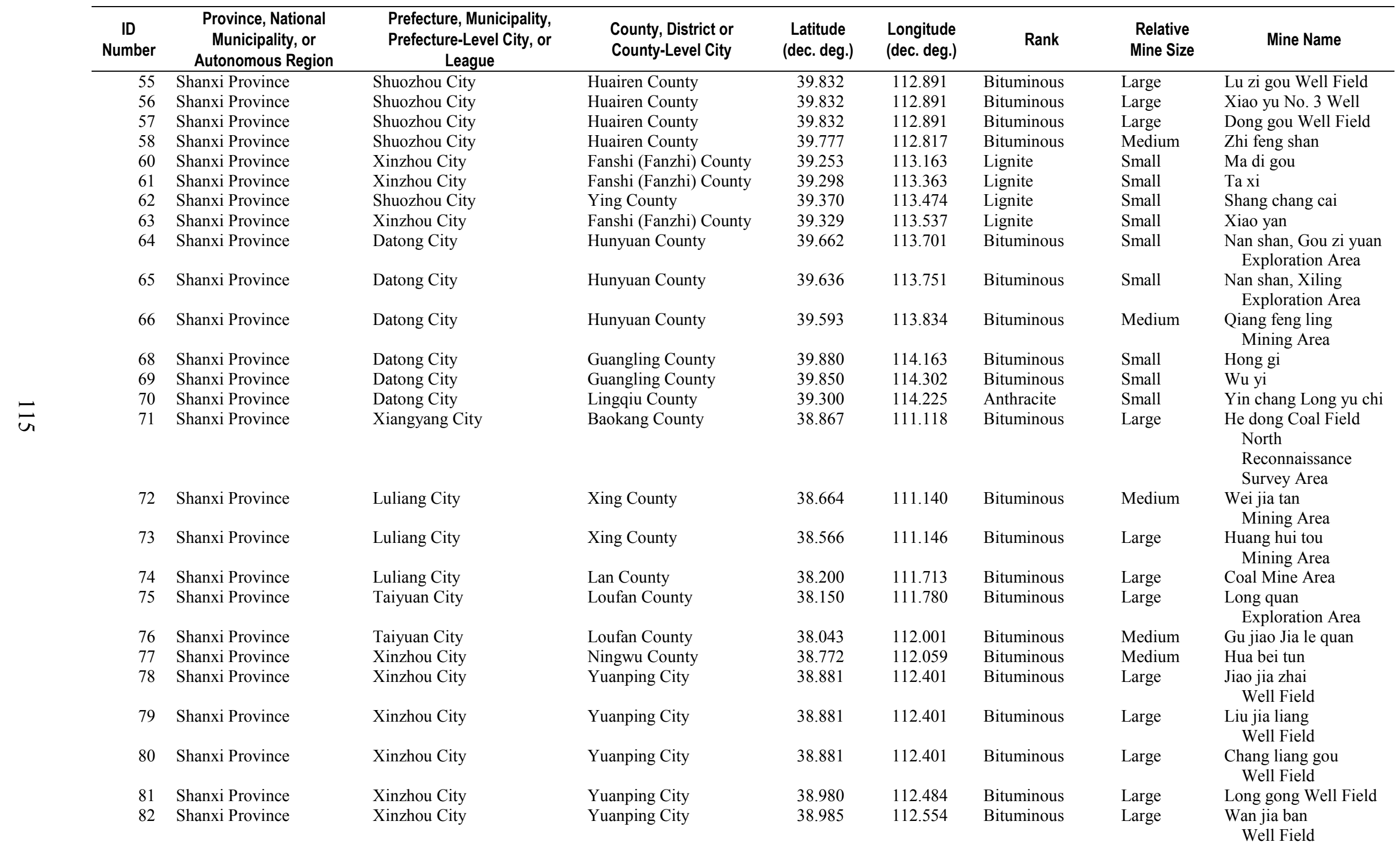


Table 1. List of coal mines in China. - Continued

[Abbreviations used: dec. deg. - decimal degrees; NA - not available or not applicable]

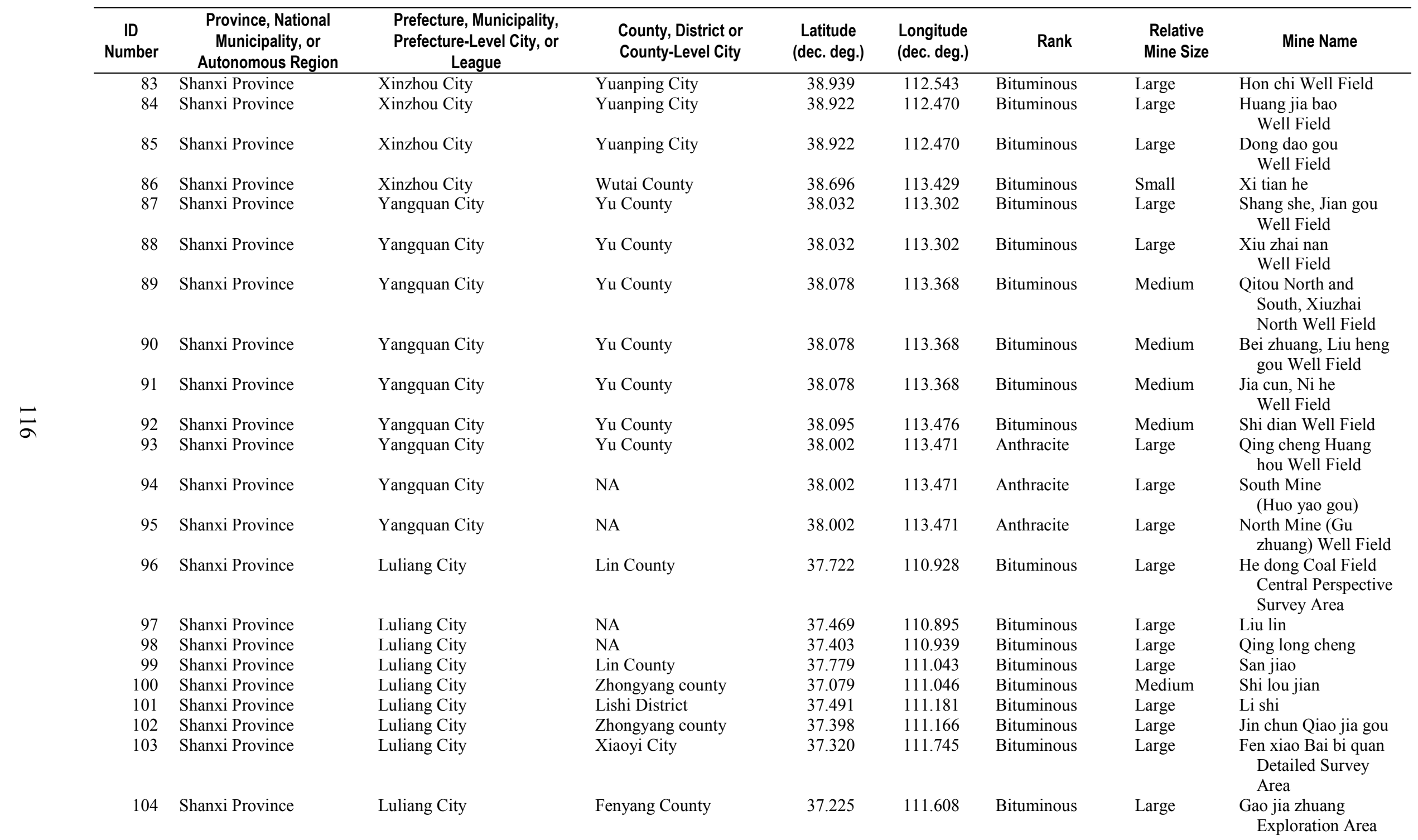


Table 1. List of coal mines in China. - Continued

[Abbreviations used: dec. deg. - decimal degrees; NA - not available or not applicable]

\begin{tabular}{|c|c|c|c|c|c|c|c|c|}
\hline $\begin{array}{c}\text { ID } \\
\text { Number }\end{array}$ & $\begin{array}{l}\text { Province, National } \\
\text { Municipality, or } \\
\text { Autonomous Region }\end{array}$ & $\begin{array}{l}\text { Prefecture, Municipality, } \\
\text { Prefecture-Level City, or } \\
\text { League }\end{array}$ & $\begin{array}{l}\text { County, District or } \\
\text { County-Level City }\end{array}$ & $\begin{array}{l}\text { Latitude } \\
\text { (dec. deg.) }\end{array}$ & $\begin{array}{l}\text { Longitude } \\
\text { (dec. deg.) }\end{array}$ & Rank & $\begin{array}{l}\text { Relative } \\
\text { Mine Size }\end{array}$ & Mine Name \\
\hline 105 & Shanxi Province & Luliang City & Fenyang County & 37.225 & 111.608 & Bituminous & Large & $\begin{array}{l}\text { Yang zhai } \\
\text { Exploration Area }\end{array}$ \\
\hline 106 & Shanxi Province & Luliang City & Xiaoyi City & 37.157 & 111.656 & Bituminous & Large & Gao yang Well Field \\
\hline 107 & Shanxi Province & Luliang City & Xiaoyi City & 37.090 & 111.582 & Bituminous & Large & Liu wan Well Field \\
\hline 108 & Shanxi Province & Luliang City & Xiaoyi City & 37.090 & 111.582 & Bituminous & Large & $\begin{array}{l}\text { Yang quan qu } \\
\text { Well Field }\end{array}$ \\
\hline 109 & Shanxi Province & Luliang City & Xiaoyi City & 37.090 & 111.582 & Bituminous & Large & Gua gou Well Field \\
\hline 110 & Shanxi Province & Luliang City & Xiaoyi City & 37.090 & 111.582 & Bituminous & Large & $\begin{array}{l}\text { Wang jia yuan } \\
\text { Well Field }\end{array}$ \\
\hline 111 & Shanxi Province & Luliang City & Xiaoyi City & 37.090 & 111.582 & Bituminous & Large & Shui yu Well Field \\
\hline 112 & Shanxi Province & Luliang City & Xiaoyi City & 37.084 & 111.744 & Bituminous & Large & Xin yao Well Field \\
\hline 113 & Shanxi Province & Luliang City & Xiaoyi City & 37.002 & 111.696 & Bituminous & Large & $\begin{array}{l}\text { Pian dian Yi tan } \\
\text { Reconnaissance } \\
\text { Exploration Area }\end{array}$ \\
\hline 114 & Shanxi Province & Luliang City & Xiaoyi City & 37.860 & 111.888 & Bituminous & Large & $\begin{array}{l}\text { Fen xiao Bai bi quan } \\
\text { East Detailed } \\
\text { Survey Area }\end{array}$ \\
\hline 115 & Shanxi Province & Jinzhong City & Jiexiu City & 37.188 & 111.868 & Bituminous & Large & Yi tang \\
\hline 116 & Shanxi Province & Taiyuan City & Gujiao City & 37.103 & 111.937 & Bituminous & Large & Ma lan Well Field \\
\hline 117 & Shanxi Province & Taiyuan City & Gujiao City & 37.958 & 112.024 & Bituminous & Large & $\begin{array}{l}\text { Zhen cheng di } \\
\text { Well Field }\end{array}$ \\
\hline 118 & Shanxi Province & Taiyuan City & Gujiao City & 37.958 & 112.024 & Bituminous & Large & Shou yu Area \\
\hline 119 & Shanxi Province & Taiyuan City & Gujiao City & 37.958 & 112.024 & Bituminous & Large & Lu yu kuo Area \\
\hline 120 & Shanxi Province & Taiyuan City & Gujiao City & 37.880 & 112.148 & Bituminous & Large & Tun lan Well Field \\
\hline 121 & Shanxi Province & Taiyuan City & Gujiao City & 37.880 & 112.148 & Bituminous & Large & Dong qu Well Field \\
\hline 122 & Shanxi Province & Taiyuan City & Gujiao City & 37.947 & 112.168 & Bituminous & Large & Xi qu Well Field \\
\hline 123 & Shanxi Province & Taiyuan City & $\mathrm{NA}$ & 37.932 & 112.366 & Bituminous & Large & Sui lao mu qu \\
\hline 124 & Shanxi Province & Taiyuan City & NA & 37.855 & 112.379 & Bituminous & Large & Xi ming Well Field \\
\hline 125 & Shanxi Province & Taiyuan City & NA & 37.855 & 112.379 & Bituminous & Large & Du er ping Well Field \\
\hline 126 & Shanxi Province & Taiyuan City & NA & 37.855 & 112.379 & Bituminous & Large & $\begin{array}{l}\text { Song shu keng } \\
\text { Well Field }\end{array}$ \\
\hline 127 & Shanxi Province & Taiyuan City & NA & 37.855 & 112.379 & Bituminous & Large & No. 2 Well Well Field \\
\hline 128 & Shanxi Province & Taiyuan City & NA & 37.855 & 112.379 & Bituminous & Large & $\begin{array}{l}\text { Xiao nan Pit } \\
\text { Well Field }\end{array}$ \\
\hline 129 & Shanxi Province & Taiyuan City & Gujiao City & 37.794 & 112.183 & Bituminous & Large & $\begin{array}{l}\text { Xing jia du } \\
\text { Reconnaissance } \\
\text { Exploration Area }\end{array}$ \\
\hline 130 & Shanxi Province & Taiyuan City & NA & 37.793 & 112.396 & Bituminous & Large & Gong di Well Field \\
\hline 131 & Shanxi Province & Taiyuan City & NA & 37.801 & 112.510 & Bituminous & Medium & $\mathrm{Xi} \mathrm{yu}$ \\
\hline
\end{tabular}


Table 1. List of coal mines in China.-Continued

[Abbreviations used: dec. deg. - decimal degrees; NA - not available or not applicable]

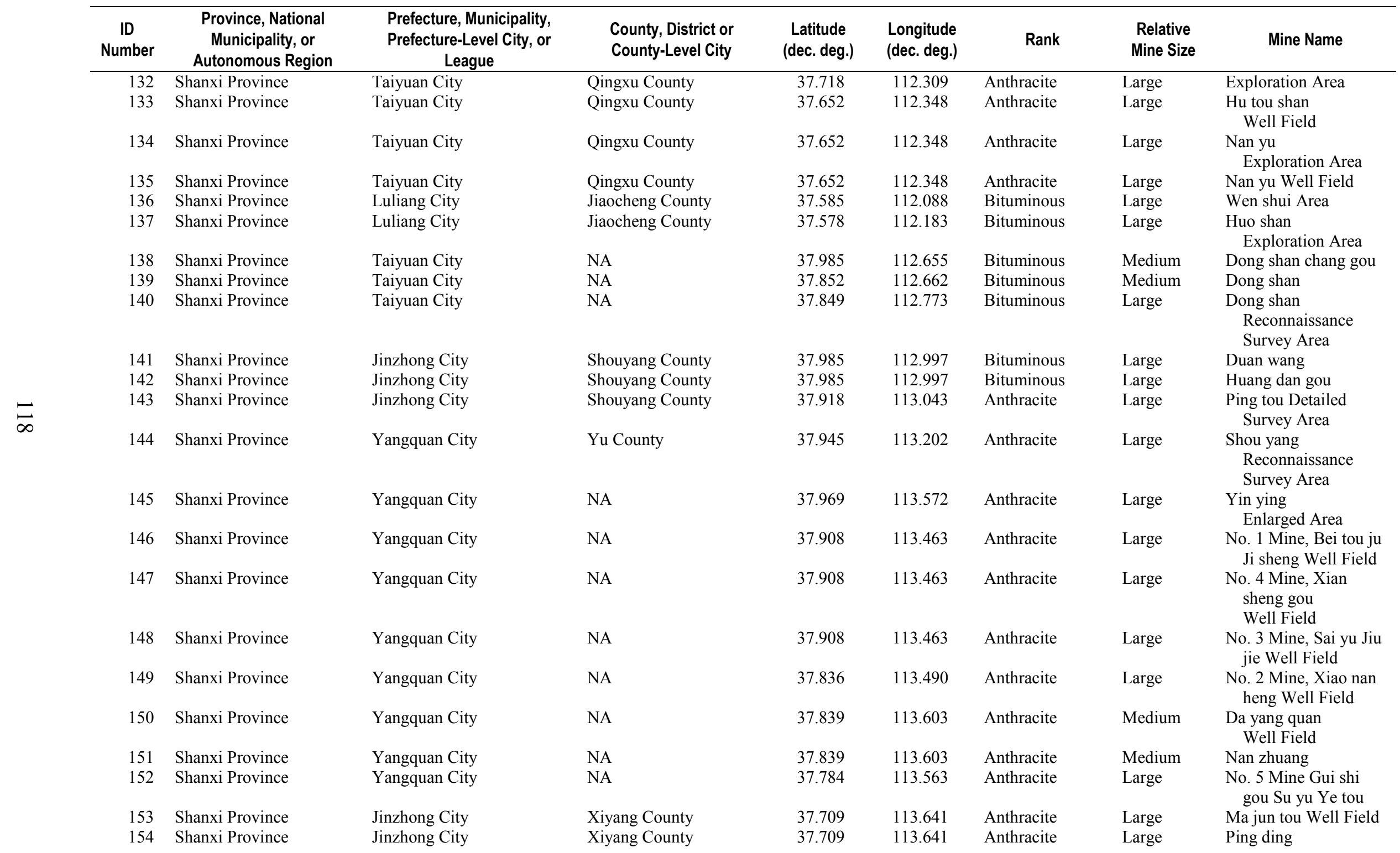


Table 1. List of coal mines in China.-Continued

[Abbreviations used: dec. deg. - decimal degrees; NA - not available or not applicable]

\begin{tabular}{|c|c|c|c|c|c|c|c|c|}
\hline $\begin{array}{l}\text { ID } \\
\text { Number }\end{array}$ & $\begin{array}{l}\text { Province, National } \\
\text { Municipality, or } \\
\text { Autonomous Region }\end{array}$ & $\begin{array}{l}\text { Prefecture, Municipality, } \\
\text { Prefecture-Level City, or } \\
\text { League }\end{array}$ & $\begin{array}{l}\text { County, District or } \\
\text { County-Level City }\end{array}$ & $\begin{array}{l}\text { Latitude } \\
\text { (dec. deg.) }\end{array}$ & $\begin{array}{l}\text { Longitude } \\
\text { (dec. deg.) }\end{array}$ & Rank & $\begin{array}{l}\text { Relative } \\
\text { Mine Size }\end{array}$ & Mine Name \\
\hline 155 & Shanxi Province & Jinzhong City & Xiyang County & 37.586 & 113.663 & Anthracite & Medium & $\begin{array}{l}\text { Zhuang zhuang } \\
\text { Well Field }\end{array}$ \\
\hline 156 & Shanxi Province & Jinzhong City & Xiyang County & 37.545 & 113.578 & Anthracite & Large & Li jia gou Well Field \\
\hline 157 & Shanxi Province & Jinzhong City & Xiyang County & 37.409 & 113.620 & Anthracite & Large & $\begin{array}{l}\text { Zhuo quan } \\
\text { Reconnaissance } \\
\text { Survey Area }\end{array}$ \\
\hline 158 & Shanxi Province & Jinzhong City & Zuoquan County & 37.049 & 113.311 & Bituminous & Large & $\begin{array}{l}\text { Wu xiang } 5 \\
\text { Exploration Area }\end{array}$ \\
\hline 159 & Shanxi Province & Jinzhong City & Zuoquan County & 36.362 & 110.953 & Bituminous & Large & $\begin{array}{l}\text { He dong Coal Field } \\
\text { South Pu xian } \\
\text { Ming zhu }\end{array}$ \\
\hline 160 & Shanxi Province & Linfen City & Xiangning County & 36.056 & 111.021 & Bituminous & Large & Zhao jia wan Area \\
\hline 161 & Shanxi Province & Luliang City & Xiaoyi City & 36.994 & 111.508 & Bituminous & Large & $\begin{array}{l}\text { Xi gou } \\
\text { Reconnaissance } \\
\text { Survey Area }\end{array}$ \\
\hline 162 & Shanxi Province & Luliang City & Xiaoyi City & 36.994 & 111.508 & Bituminous & Large & $\begin{array}{l}\text { Jiao zi li } \\
\quad \text { Exploration Area }\end{array}$ \\
\hline 163 & Shanxi Province & Jinzhong City & Jiexiu City & 36.948 & 111.697 & Bituminous & Medium & Da fo si Well Field \\
\hline 164 & Shanxi Province & Jinzhong City & Jiexiu City & 36.973 & 111.908 & Bituminous & Large & $\begin{array}{l}\text { Jie nan } \\
\quad \text { Reconnaissance } \\
\text { Exploration Area }\end{array}$ \\
\hline 165 & Shanxi Province & Luliang City & Jiaokou County & 36.834 & 111.482 & Bituminous & Large & $\begin{array}{l}\text { Shuang chi } \\
\text { Reconnaissance } \\
\text { Exploration Area }\end{array}$ \\
\hline 166 & Shanxi Province & Jinzhong City & Lingshi County & 36.879 & 111.681 & Bituminous & Large & Cui jia gou Well Field \\
\hline 167 & Shanxi Province & Jinzhong City & Lingshi County & 36.866 & 111.794 & Bituminous & Medium & Ling shi Mining Area \\
\hline 168 & Shanxi Province & Jinzhong City & Lingshi County & 36.903 & 111.856 & Bituminous & Medium & $\begin{array}{l}\text { You jia shan } \\
\text { Exploration Area }\end{array}$ \\
\hline 169 & Shanxi Province & Jinzhong City & Lingshi County & 36.832 & 111.834 & Bituminous & Large & $\begin{array}{l}\text { Cao qiao gou } \\
\text { Exploration Area }\end{array}$ \\
\hline 170 & Shanxi Province & Jinzhong City & Lingshi County & 36.716 & 111.518 & Bituminous & Large & Dao mei Mining Area \\
\hline 171 & Shanxi Province & Linfen City & Huozhou City & 36.678 & 111.806 & Bituminous & Large & $\begin{array}{l}\text { Li ya zhuang } \\
\text { Well Field }\end{array}$ \\
\hline 172 & Shanxi Province & Linfen City & Fenxi County & 36.626 & 111.694 & Bituminous & Large & $\begin{array}{l}\text { Wa yao qi ta } \\
\text { Well Field }\end{array}$ \\
\hline 173 & Shanxi Province & Linfen City & Huozhou City & 36.626 & 111.694 & Bituminous & Large & Shi lin Well Field \\
\hline 174 & Shanxi Province & Linfen City & Huozhou City & 36.626 & 111.694 & Bituminous & Large & Tui sha Area \\
\hline 175 & Shanxi Province & Linfen City & $\mathrm{Pu}$ County & 36.509 & 111.283 & Bituminous & Medium & $\begin{array}{l}\text { Dong he Detailed } \\
\text { suvey Area }\end{array}$ \\
\hline
\end{tabular}


Table 1. List of coal mines in China. - Continued

[Abbreviations used: dec. deg. - decimal degrees; NA - not available or not applicable]

\begin{tabular}{|c|c|c|c|c|c|c|c|c|}
\hline $\begin{array}{c}\text { ID } \\
\text { Number }\end{array}$ & $\begin{array}{l}\text { Province, National } \\
\text { Municipality, or } \\
\text { Autonomous Region }\end{array}$ & $\begin{array}{c}\text { Prefecture, Municipality, } \\
\text { Prefecture-Level City, or } \\
\text { League }\end{array}$ & $\begin{array}{l}\text { County, District or } \\
\text { County-Level City }\end{array}$ & $\begin{array}{l}\text { Latitude } \\
\text { (dec. deg.) }\end{array}$ & $\begin{array}{l}\text { Longitude } \\
\text { (dec. deg.) }\end{array}$ & Rank & $\begin{array}{l}\text { Relative } \\
\text { Mine Size }\end{array}$ & Mine Name \\
\hline 178 & Shanxi Province & Linfen City & Fenxi County & 36.496 & 111.605 & Bituminous & Large & Tuan pai Well Field \\
\hline 179 & Shanxi Province & Linfen City & Huozhou City & 36.540 & 111.647 & Bituminous & Large & Bai long Well Field \\
\hline 180 & Shanxi Province & Linfen City & Huozhou City & 36.507 & 111.753 & Bituminous & Large & Bei yi chang Area \\
\hline 181 & Shanxi Province & Linfen City & Huozhou City & 36.507 & 111.753 & Bituminous & Large & Sheng fo \\
\hline 182 & Shanxi Province & Linfen City & Huozhou City & 36.507 & 111.753 & Bituminous & Large & $\begin{array}{l}\text { Nan xia zhuang, } \\
\text { Dong xi Mine }\end{array}$ \\
\hline 183 & Shanxi Province & Linfen City & Huozhou City & 36.507 & 111.753 & Bituminous & Large & Cao cun Well Field \\
\hline 184 & Shanxi Province & Linfen City & Huozhou City & 36.507 & 111.753 & Bituminous & Large & $\begin{array}{l}\text { Hou zhou, outskirts of } \\
\text { Mining Area }\end{array}$ \\
\hline 185 & Shanxi Province & Linfen City & Huozhou City & 36.507 & 111.753 & Bituminous & Large & Xia le ping \\
\hline 186 & Shanxi Province & Linfen City & Huozhou City & 36.507 & 111.753 & Bituminous & Large & $\begin{array}{l}\text { Xin zhi } \\
\text { Well Field North }\end{array}$ \\
\hline 187 & Shanxi Province & Linfen City & $\mathrm{Pu}$ County & 36.446 & 111.307 & Bituminous & Large & Ke cheng \\
\hline 189 & Shanxi Province & Linfen City & Hongdong County & 36.443 & 111.629 & Bituminous & Large & $\begin{array}{l}\text { Wa nan } \\
\text { Reconnaissance } \\
\text { Exploration Area }\end{array}$ \\
\hline 190 & Shanxi Province & Linfen City & Huozhou City & 36.463 & 111.769 & Bituminous & Medium & $\begin{array}{l}\text { Xin zhi } \\
\text { Well Field South }\end{array}$ \\
\hline 191 & Shanxi Province & Linfen City & NA & 36.221 & 111.314 & Bituminous & Large & $\begin{array}{l}\text { Nan wan Ii } \\
\text { Reconnaissnce } \\
\text { Survey Area }\end{array}$ \\
\hline 192 & Shanxi Province & Linfen City & NA & 36.241 & 111.514 & Bituminous & Small & Tu men West \\
\hline 193 & Shanxi Province & Linfen City & Hongdong County & 36.294 & 111.802 & Bituminous & Small & $\begin{array}{l}\text { Guang sheng si } \\
\text { Exploration Area }\end{array}$ \\
\hline 194 & Shanxi Province & Changzhi City & Qinyuan County & 36.659 & 112.185 & Bituminous & Large & $\begin{array}{l}\text { Huo dong, } \\
\text { Zhao mei qu }\end{array}$ \\
\hline 195 & Shanxi Province & Changzhi City & Qinyuan County & 36.817 & 112.273 & Bituminous & Large & $\begin{array}{l}\text { Reconnaissance } \\
\text { Exploration Area }\end{array}$ \\
\hline 196 & Shanxi Province & Changzhi City & Tunliu County & 36.347 & 112.706 & Anthracite & Large & $\begin{array}{l}\text { Tun liu Detailed } \\
\text { Survey Area }\end{array}$ \\
\hline 197 & Shanxi Province & Changzhi City & Xiangyuan County & 36.559 & 112.965 & Bituminous & Large & $\begin{array}{l}\text { Xia dian Detailed } \\
\text { Survey Area }\end{array}$ \\
\hline 198 & Shanxi Province & Changzhi City & Xiangyuan County & 36.559 & 112.965 & Bituminous & Large & Xiang yuan \\
\hline 199 & Shanxi Province & Changzhi City & Xiangyuan County & 36.488 & 112.996 & Bituminous & Large & $\begin{array}{l}\text { Nan feng Detailed } \\
\text { Survey Area }\end{array}$ \\
\hline 200 & Shanxi Province & Changzhi City & Xiangyuan County & 36.488 & 112.996 & Bituminous & Large & Wu yang \\
\hline 201 & Shanxi Province & Changzhi City & Tunliu County & 36.376 & 112.975 & Anthracite & Large & $\begin{array}{l}\text { Guang zhuang } \\
\text { Well Field }\end{array}$ \\
\hline 202 & Shanxi Province & Changzhi City & Tunliu County & 36.376 & 112.975 & Bituminous & Large & Chang cun Well Field \\
\hline
\end{tabular}


Table 1. List of coal mines in China.-Continued

[Abbreviations used: dec. deg. - decimal degrees; NA - not available or not applicable]

\begin{tabular}{|c|c|c|c|c|c|c|c|c|}
\hline $\begin{array}{c}\text { ID } \\
\text { Number }\end{array}$ & $\begin{array}{l}\text { Province, National } \\
\text { Municipality, or } \\
\text { Autonomous Region }\end{array}$ & $\begin{array}{c}\text { Prefecture, Municipality, } \\
\text { Prefecture-Level City, or } \\
\text { League }\end{array}$ & $\begin{array}{l}\text { County, District or } \\
\text { County-Level City }\end{array}$ & $\begin{array}{l}\text { Latitude } \\
\text { (dec. deg.) }\end{array}$ & $\begin{array}{l}\text { Longitude } \\
\text { (dec. deg.) }\end{array}$ & Rank & $\begin{array}{l}\text { Relative } \\
\text { Mine Size }\end{array}$ & Mine Name \\
\hline 203 & Shanxi Province & Changzhi City & NA & 36.376 & 112.975 & Bituminous & Large & Wang zhuang \\
\hline 204 & Shanxi Province & Changzhi City & NA & 36.154 & 112.977 & Bituminous & Large & $\begin{array}{l}\text { Chang zi } \\
\text { Reconnaissance } \\
\text { Survey Area }\end{array}$ \\
\hline 205 & Shanxi Province & Changzhi City & NA & 36.154 & 112.977 & Anthracite & Large & Detailed Survey Area \\
\hline 206 & Shanxi Province & Changzhi City & NA & 36.154 & 112.977 & Bituminous & Large & Nan zhai Well Field \\
\hline 207 & Shanxi Province & Changzhi City & Wuxiang County & 36.968 & 113.262 & Bituminous & Large & 3-4 Exploration Area \\
\hline 208 & Shanxi Province & Changzhi City & Wuxiang County & 36.857 & 113.197 & Bituminous & Large & 1-2 Exploration Area \\
\hline 209 & Shanxi Province & Changzhi City & Xiangyuan County & 36.697 & 113.037 & Bituminous & Large & $\begin{array}{l}\text { Xiang yuan } \\
\text { Reconnaissance } \\
\text { Survey Area }\end{array}$ \\
\hline 210 & Shanxi Province & Changzhi City & NA & 36.446 & 113.074 & Bituminous & Medium & Zhang cun \\
\hline 211 & Shanxi Province & Changzhi City & NA & 36.402 & 113.085 & Bituminous & Large & Shi qi jie \\
\hline 212 & Shanxi Province & Yuncheng City & Hejin City & 35.703 & 110.616 & Bituminous & Small & Du jia gou \\
\hline 213 & Shanxi Province & Linfen City & Xiangning County & 35.837 & 110.723 & Bituminous & Large & $\begin{array}{l}\text { Wang jia ling } \\
\text { Well Field }\end{array}$ \\
\hline 214 & Shanxi Province & Linfen City & Xiangning County & 35.966 & 110.917 & Bituminous & Large & $\begin{array}{l}\text { Zhao jia wan } \\
\text { Reconnaissance } \\
\text { Survev Area }\end{array}$ \\
\hline 215 & Shanxi Province & Linfen City & Xiangfen County & 35.939 & 111.381 & Bituminous & Large & $\begin{array}{l}\text { Xiang fen } \\
\text { Exploration Area }\end{array}$ \\
\hline 216 & Shanxi Province & Linfen City & Yicheng County & 35.765 & 111.867 & Bituminous & Medium & Lao zhai \\
\hline 217 & Shanxi Province & Linfen City & Yicheng County & 35.571 & 111.958 & Anthracite & Large & Xi yan \\
\hline 218 & Shanxi Province & Linfen City & Yicheng County & 35.811 & 112.050 & Anthracite & Large & Long hua \\
\hline 219 & Shanxi Province & Jinzhong City & Qinshui County & 35.677 & 112.173 & Anthracite & Large & $\begin{array}{l}\text { Qin shui } \\
\text { Reconnaissance } \\
\text { Survey Area }\end{array}$ \\
\hline 220 & Shanxi Province & Changzhi City & Zhangzi County & 35.945 & 112.794 & Anthracite & Large & Bu cun - Zhang dian \\
\hline 221 & Shanxi Province & Changzhi City & Zhangzi County & 35.978 & 112.905 & Anthracite & Medium & Ci lin shan \\
\hline 222 & Shanxi Province & Changzhi City & Zhangzi County & 35.932 & 112.898 & Anthracite & Medium & Se tou \\
\hline 223 & Shanxi Province & Jincheng City & Gaoping County & 35.896 & 112.878 & Anthracite & Medium & Wang yun Well Field \\
\hline 224 & Shanxi Province & Jincheng City & Gaoping County & 35.885 & 112.781 & Anthracite & Large & Wang bao \\
\hline 225 & Shanxi Province & Jincheng City & Gaoping County & 35.834 & 112.834 & Anthracite & Large & $\begin{array}{c}\text { Reconnaissance } \\
\text { Survey Area }\end{array}$ \\
\hline 226 & Shanxi Province & Jincheng City & Gaoping County & 35.802 & 112.967 & Anthracite & Medium & Yue zhuang \\
\hline 227 & Shanxi Province & Jincheng City & Yangcheng County & 35.742 & 112.624 & Anthracite & Large & $\begin{array}{l}\text { Reconnaissance } \\
\text { Survey Area }\end{array}$ \\
\hline 228 & Shanxi Province & Jincheng City & Gaoping County & 35.755 & 112.815 & Anthracite & Large & Ye chuan Well Field \\
\hline
\end{tabular}


Table 1. List of coal mines in China. - Continued

[Abbreviations used: dec. deg. - decimal degrees; NA - not available or not applicable]

\begin{tabular}{|c|c|c|c|c|c|c|c|c|}
\hline $\begin{array}{c}\text { ID } \\
\text { Number }\end{array}$ & $\begin{array}{l}\text { Province, National } \\
\text { Municipality, or } \\
\text { Autonomous Region }\end{array}$ & $\begin{array}{l}\text { Prefecture, Municipality, } \\
\text { Prefecture-Level City, or } \\
\text { League }\end{array}$ & $\begin{array}{l}\text { County, District or } \\
\text { County-Level City }\end{array}$ & $\begin{array}{l}\text { Latitude } \\
\text { (dec. deg.) }\end{array}$ & $\begin{array}{l}\text { Longitude } \\
\text { (dec. deg.) }\end{array}$ & Rank & $\begin{array}{l}\text { Relative } \\
\text { Mine Size }\end{array}$ & Mine Name \\
\hline 229 & Shanxi Province & Jincheng City & Gaoping County & 35.756 & 112.911 & Anthracite & Large & $\begin{array}{l}\text { Niu shan } \\
\quad \text { Exploration Area }\end{array}$ \\
\hline 230 & Shanxi Province & Jincheng City & NA & 35.696 & 112.760 & Anthracite & Large & Da yan Well Field \\
\hline 231 & Shanxi Province & Jincheng City & NA & 35.680 & 112.931 & Anthracite & Medium & Ju shan Mining Well \\
\hline 232 & Shanxi Province & Jincheng City & NA & 35.680 & 112.931 & Anthracite & Medium & No. 38 Coal Mine \\
\hline 233 & Shanxi Province & Jincheng City & Gaoping County & 35.683 & 112.929 & Anthracite & Medium & $\begin{array}{l}\text { Niu cun Well Field, } \\
\text { Xin zhuang }\end{array}$ \\
\hline 234 & Shanxi Province & Jincheng City & Yangcheng County & 35.584 & 112.457 & Anthracite & Large & $\begin{array}{l}\text { Shang huang ya } \\
\text { Well Field }\end{array}$ \\
\hline 235 & Shanxi Province & Jincheng City & Yangcheng County & 35.584 & 112.457 & Anthracite & Large & $\begin{array}{l}\text { Tian ding dian } \\
\text { Exploration Area }\end{array}$ \\
\hline 236 & Shanxi Province & Jincheng City & Qinshui County & 35.629 & 112.583 & Anthracite & Large & Yang cheng, Chang he \\
\hline 237 & Shanxi Province & Jincheng City & Qinshui County & 35.629 & 112.583 & Anthracite & Large & $\begin{array}{l}\text { Pan zhang No. } 1 \\
\text { Well Field }\end{array}$ \\
\hline 238 & Shanxi Province & Jincheng City & NA & 35.632 & 112.716 & Anthracite & Large & $\begin{array}{l}\text { Cheng zhuang } \\
\text { Well Field }\end{array}$ \\
\hline 239 & Shanxi Province & Jincheng City & NA & 35.616 & 112.932 & Anthracite & Large & Feng huang shan \\
\hline 240 & Shanxi Province & Jincheng City & NA & 35.532 & 112.608 & Anthracite & Large & Si he Well Field \\
\hline 241 & Shanxi Province & Jincheng City & NA & 35.551 & 112.867 & Anthracite & Large & Bei yan \\
\hline 242 & Shanxi Province & Jincheng City & NA & 35.551 & 112.867 & Anthracite & Large & $\begin{array}{l}\text { Si xin Mine, } \\
\text { Gu shu yuan }\end{array}$ \\
\hline 243 & Shanxi Province & Jincheng City & NA & 35.551 & 112.867 & Anthracite & Large & Wang tai pu \\
\hline 244 & Shanxi Province & Jincheng City & NA & 35.454 & 112.748 & Anthracite & Large & Jin pu shan Well Field \\
\hline 245 & Shanxi Province & Jincheng City & NA & 35.406 & 112.748 & Anthracite & Medium & Jin pu Southern Area \\
\hline 246 & Shanxi Province & Changzhi City & NA & 35.932 & 113.073 & Bituminous & Large & Wang zhang \\
\hline 247 & Shanxi Province & Jincheng City & Lingchuan County & 35.810 & 113.300 & Anthracite & Large & $\begin{array}{l}\text { Ling chuan } \\
\text { Reconnaissance } \\
\text { Survey Area } \\
\end{array}$ \\
\hline 30 & Sichuan Province & Guangyuan City & NA & 32.374 & 106.025 & Bituminous & Small & Yu jia bian \\
\hline 31 & Sichuan Province & Guangyuan City & Wancang County & 32.286 & 106.360 & Bituminous & Small & Tan jia he \\
\hline 32 & Sichuan Province & Guangyuan City & Wancang County & 32.278 & 106.510 & Bituminous & Small & Dai chi ba \\
\hline 33 & Sichuan Province & Guangyuan City & Wancang County & 32.276 & 106.638 & Bituminous & Small & Shi dong gou \\
\hline 34 & Sichuan Province & Bazhong City & Nanjiang County & 32.338 & 106.685 & Bituminous & Small & Shui dong \\
\hline 38 & Sichuan Province & $\begin{array}{l}\text { Garze Autonomous } \\
\text { Prefecture }\end{array}$ & Baiyu County & 31.029 & 99.525 & Lignite & Small & Chang tai \\
\hline 43 & Sichuan Province & Chengdu City & Dayi County & 30.638 & 103.330 & Bituminous & Small & Shen xian qiao \\
\hline 44 & Sichuan Province & Chengdu City & Chongzhou County & 30.815 & 103.418 & Bituminous & Small & Fang dian zi \\
\hline 50 & Sichuan Province & Chengdu City & Pengzhou City & 31.156 & 103.896 & Bituminous & Small & Bai ma miao \\
\hline 51 & Sichuan Province & Deyang City & Mianzhu City & 31.461 & 104.139 & Bituminous & Small & He jia shan \\
\hline
\end{tabular}


Table 1. List of coal mines in China. - Continued

[Abbreviations used: dec. deg. - decimal degrees; NA - not available or not applicable]

\begin{tabular}{|c|c|c|c|c|c|c|c|c|}
\hline $\begin{array}{c}\text { ID } \\
\text { Number }\end{array}$ & $\begin{array}{c}\text { Province, National } \\
\text { Municipality, or } \\
\text { Autonomous Region }\end{array}$ & $\begin{array}{c}\text { Prefecture, Municipality, } \\
\text { Prefecture-Level City, or } \\
\text { League }\end{array}$ & $\begin{array}{l}\text { County, District or } \\
\text { County-Level City }\end{array}$ & $\begin{array}{l}\text { Latitude } \\
\text { (dec. deg.) }\end{array}$ & $\begin{array}{l}\text { Longitude } \\
\text { (dec. deg.) }\end{array}$ & Rank & $\begin{array}{l}\text { Relative } \\
\text { Mine Size }\end{array}$ & Mine Name \\
\hline 52 & Sichuan Province & Dazhou City & Xuanhan County & 31.262 & 107.808 & Bituminous & Small & Lu guang ping \\
\hline 53 & Sichuan Province & Dazhou City & Xuanhan County & 31.201 & 107.758 & Bituminous & Small & Da lu gou \\
\hline 54 & Sichuan Province & Dazhou City & Kaijiang County & 31.144 & 107.697 & Bituminous & Small & Kai feng \\
\hline 55 & Sichuan Province & Dazhou City & Da County & 31.083 & 107.388 & Bituminous & Small & Jin wo \\
\hline 56 & Sichuan Province & Dazhou City & Da County & 31.099 & 107.482 & Bituminous & Small & Yan tan wan \\
\hline 57 & Sichuan Province & Dazhou City & Da County & 31.013 & 107.331 & Bituminous & Small & Tie shan nan \\
\hline 58 & Sichuan Province & Qingshui City & Dazhu County & 30.965 & 107.174 & Bituminous & Small & Bo lin \\
\hline 59 & Sichuan Province & Dazhou City & Da County & 30.950 & 107.478 & Bituminous & Small & Well Field \\
\hline 60 & Sichuan Province & Dazhou City & Da County & 30.896 & 107.413 & Bituminous & Small & Bin lang \\
\hline 61 & Sichuan Province & Dazhou City & Da County & 30.830 & 107.398 & Bituminous & Medium & Jin gang \\
\hline 62 & Sichuan Province & Qingshui City & Dazhu County & 30.805 & 107.119 & Bituminous & Small & Li zhu si \\
\hline 63 & Sichuan Province & Qingshui City & Dazhu County & 30.767 & 107.329 & Bituminous & Small & Wei jia shan \\
\hline 64 & Sichuan Province & Qingshui City & Dazhu County & 30.704 & 107.314 & Bituminous & Small & Bao zi \\
\hline 65 & Sichuan Province & Qingshui City & Dazhu County & 30.693 & 107.075 & Bituminous & Small & Long men xia \\
\hline 66 & Sichuan Province & Qingshui City & Dazhu County & 30.639 & 107.276 & Bituminous & Small & Tuan ba \\
\hline 67 & Sichuan Province & Guang'an City & Huaying City & 30.442 & 106.843 & Bituminous & Small & Lu shui dong \\
\hline 68 & Sichuan Province & Guang'an City & Huaying City & 30.462 & 106.948 & Bituminous & Medium & Long tan \\
\hline 69 & Sichuan Province & Guang'an City & Linshui County & 30.408 & 106.847 & Bituminous & Small & $\mathrm{Xi}$ tian si \\
\hline 70 & Sichuan Province & Guang'an City & Linshui County & 30.408 & 106.872 & Bituminous & Small & Shi yan kou \\
\hline 71 & Sichuan Province & Guang'an City & Huaying City & 30.340 & 106.861 & Bituminous & Small & Li jia gou \\
\hline 72 & Sichuan Province & Guang'an City & Huaying City & 30.316 & 106.777 & Bituminous & Small & Gao ding shan \\
\hline 73 & Sichuan Province & Guang'an City & NA & 30.355 & 107.012 & Bituminous & Small & Da tian wan \\
\hline 74 & Sichuan Province & Guang'an City & Huaying City & 30.303 & 106.962 & Bituminous & Small & Gong qiao wan \\
\hline 75 & Sichuan Province & Guang'an City & Huaying City & 30.243 & 106.911 & Bituminous & Small & Gui xing \\
\hline 76 & Sichuan Province & Guang'an City & Huaying City & 30.217 & 106.759 & Bituminous & Medium & Li zie \\
\hline 77 & Sichuan Province & Guang'an City & Huaying City & 30.129 & 106.681 & Bituminous & Small & $\mathrm{Xi} \mathrm{kou}$ \\
\hline 78 & Sichuan Province & Guang'an City & Huaying City & 30.160 & 106.737 & Bituminous & Small & Hua ying shan \\
\hline 88 & Sichuan Province & Leshan City & NA & 29.376 & 103.565 & Bituminous & Small & Hong yuan \\
\hline 89 & Sichuan Province & Leshan City & $\begin{array}{l}\text { Qianwei (Jianwei) } \\
\text { County }\end{array}$ & 29.472 & 103.963 & Bituminous & Small & Wei gan ba \\
\hline 90 & Sichuan Province & Leshan City & $\begin{array}{l}\text { Qianwei (Jianwei) } \\
\text { County }\end{array}$ & 29.424 & 103.976 & Bituminous & Small & Liang he kou \\
\hline 91 & Sichuan Province & Leshan City & NA & 29.365 & 103.886 & Bituminous & Small & San cha he \\
\hline 92 & Sichuan Province & Leshan City & $\begin{array}{l}\text { Qianwei (Jianwei) } \\
\text { County }\end{array}$ & 29.310 & 103.835 & Bituminous & Small & Tian xi \\
\hline 93 & Sichuan Province & Leshan City & Emeishan City & 29.107 & 103.612 & Bituminous & Small & Yang cun pu \\
\hline 94 & Sichuan Province & Neijiang City & Weiyuan County & 29.704 & 104.381 & Bituminous & Small & Xiang jia zhai \\
\hline 95 & Sichuan Province & Neijiang City & Zizhong County & 29.751 & 104.744 & Bituminous & Small & Lao ying yan \\
\hline 96 & Sichuan Province & Neijiang City & Zizhong County & 29.737 & 104.812 & Bituminous & Small & Nan si \\
\hline
\end{tabular}


Table 1. List of coal mines in China.-Continued

[Abbreviations used: dec. deg. - decimal degrees; NA - not available or not applicable]

\begin{tabular}{|c|c|c|c|c|c|c|c|c|}
\hline $\begin{array}{c}\text { ID } \\
\text { Number }\end{array}$ & $\begin{array}{c}\text { Province, National } \\
\text { Municipality, or } \\
\text { Autonomous Region }\end{array}$ & $\begin{array}{c}\text { Prefecture, Municipality, } \\
\text { Prefecture-Level City, or } \\
\text { League }\end{array}$ & $\begin{array}{l}\text { County, District or } \\
\text { County-Level City }\end{array}$ & $\begin{array}{l}\text { Latitude } \\
\text { (dec. deg.) }\end{array}$ & $\begin{array}{l}\text { Longitude } \\
\text { (dec. deg.) }\end{array}$ & Rank & $\begin{array}{l}\text { Relative } \\
\text { Mine Size }\end{array}$ & Mine Name \\
\hline 97 & Sichuan Province & Neijiang City & Weiyuan County & 29.658 & 104.691 & Bituminous & Small & Huang jing gou \\
\hline 102 & Sichuan Province & Chongqing City & Rongchang County & 29.277 & 105.468 & Bituminous & Small & $\begin{array}{l}\text { Shuang he } \\
\text { No. } 9 \text { \& } 10 \text { Well }\end{array}$ \\
\hline 103 & Sichuan Province & Luzhou City & Lu County & 29.048 & 105.449 & Bituminous & Small & Hu li po \\
\hline 104 & Sichuan Province & Yibin City & Gao County & 28.454 & 104.671 & Bituminous & Medium & Teng long Gui hua \\
\hline 105 & Sichuan Province & Yibin City & Gong County & 28.409 & 104.909 & Anthracite & Medium & $\begin{array}{l}\text { Fu rong shan Coal } \\
\text { Mining Area }\end{array}$ \\
\hline 106 & Sichuan Province & Yibin City & Julian (Yunlian) County & 28.011 & 104.680 & Anthracite & Large & Coal Mining Area \\
\hline 107 & Sichuan Province & Luzhou City & Gulin County & 28.017 & 105.963 & Anthracite & Large & $\begin{array}{l}\text { Gu xu Coal } \\
\text { Mining Area }\end{array}$ \\
\hline 130 & Sichuan Province & $\begin{array}{l}\text { Liangshan Autonomous } \\
\text { Prefecture }\end{array}$ & Yanyuan County & 27.478 & 101.147 & Lignite & Small & Qing shui he \\
\hline 131 & Sichuan Province & $\begin{array}{l}\text { Liangshan Autonomous } \\
\text { Prefecture }\end{array}$ & Yanyuan County & 27.481 & 101.264 & Lignite & Medium & Mei yu \\
\hline 132 & Sichuan Province & Panzhihua City & Yanbian County & 27.399 & 101.281 & Lignite & Large & He shao \\
\hline 133 & Sichuan Province & Panzhihua City & Yanbian County & 26.786 & 101.655 & Bituminous & Small & $\begin{array}{l}\text { Mo shi qing } \\
\text { No. } 12 \& 3 \text { Well }\end{array}$ \\
\hline 134 & Sichuan Province & Panzhihua City & Yanbian County & 26.777 & 101.645 & Bituminous & Small & Hong guo \\
\hline 135 & Sichuan Province & $\begin{array}{l}\text { Liangshan Autonomous } \\
\text { Prefecture }\end{array}$ & Huili County & 26.781 & 102.204 & Bituminous & Small & Wang jia ping \\
\hline 136 & Sichuan Province & $\begin{array}{l}\text { Liangshan Autonomous } \\
\text { Prefecture }\end{array}$ & Huili County & 26.736 & 102.178 & Bituminous & Small & Lao shan ping \\
\hline 137 & Sichuan Province & Panzhihua City & NA & 26.560 & 101.543 & Bituminous & Medium & Hua shan \\
\hline 138 & Sichuan Province & Panzhihua City & NA & 26.564 & 101.565 & Bituminous & Small & Yan jiang \\
\hline 139 & Sichuan Province & Panzhihua City & NA & 26.556 & 101.594 & Bituminous & Medium & Xiao bao ding \\
\hline 140 & Sichuan Province & Panzhihua City & NA & 26.537 & 101.530 & Bituminous & Medium & Tai ping \\
\hline 141 & Sichuan Province & Panzhihua City & NA & 26.533 & 101.559 & Bituminous & Medium & Da bao ding \\
\hline 142 & Sichuan Province & Panzhihua City & NA & 26.535 & 101.592 & Bituminous & Small & Da qiao di \\
\hline 12 & $\begin{array}{l}\text { Taiwan } \\
\text { (Republic of China) }\end{array}$ & Keelung (Jilong) City & NA & 25.146 & 121.783 & Bituminous & Small & Tian liao gang \\
\hline 13 & $\begin{array}{l}\text { Taiwan } \\
\quad \text { (Republic of China) }\end{array}$ & Keelung (Jilong) City & NA & 25.127 & 121.797 & Bituminous & Small & Si jiao ting \\
\hline 14 & $\begin{array}{l}\text { Taiwan } \\
\text { (Republic of China) }\end{array}$ & Taipei City & NA & 25.100 & 121.657 & Bituminous & Medium & $\mathrm{Xi}$ zhi \\
\hline 15 & $\begin{array}{l}\text { Taiwan } \\
\quad \text { (Republic of China) }\end{array}$ & Taipei City & NA & 25.077 & 121.792 & Bituminous & Small & Hou dong \\
\hline 16 & $\begin{array}{l}\text { Taiwan } \\
\text { (Republic of China) }\end{array}$ & Taipei City & NA & 25.070 & 121.709 & Bituminous & Small & Ba fen liao \\
\hline
\end{tabular}


Table 1. List of coal mines in China.-Continued

[Abbreviations used: dec. deg. - decimal degrees; NA - not available or not applicable]

\begin{tabular}{|c|c|c|c|c|c|c|c|c|}
\hline $\begin{array}{c}\text { ID } \\
\text { Number }\end{array}$ & $\begin{array}{c}\text { Province, National } \\
\text { Municipality, or } \\
\text { Autonomous Region }\end{array}$ & $\begin{array}{c}\text { Prefecture, Municipality, } \\
\text { Prefecture-Level City, or } \\
\text { League }\end{array}$ & $\begin{array}{l}\text { County, District or } \\
\text { County-Level City }\end{array}$ & $\begin{array}{l}\text { Latitude } \\
\text { (dec. deg.) }\end{array}$ & $\begin{array}{l}\text { Longitude } \\
\text { (dec. deg.) }\end{array}$ & Rank & $\begin{array}{l}\text { Relative } \\
\text { Mine Size }\end{array}$ & Mine Name \\
\hline 17 & $\begin{array}{l}\text { Taiwan } \\
\quad \text { (Republic of China) }\end{array}$ & Taipei City & NA & 25.049 & 121.815 & Bituminous & Small & Ding shuang xi \\
\hline 18 & $\begin{array}{l}\text { Taiwan } \\
\text { (Republic of China) }\end{array}$ & Taipei City & NA & 25.040 & 121.629 & Bituminous & Small & Nan gang \\
\hline 19 & $\begin{array}{l}\text { Taiwan } \\
\quad \text { (Republic of China) }\end{array}$ & Taipei City & NA & 25.033 & 121.730 & Bituminous & Small & $\begin{array}{l}\text { Qing tong keng } \\
\text { (Qing tong Pit) }\end{array}$ \\
\hline 20 & $\begin{array}{l}\text { Taiwan } \\
\quad \text { (Republic of China) }\end{array}$ & Taipei City & NA & 25.032 & 121.766 & Bituminous & Small & $\begin{array}{l}\text { Wu dan keng } \\
\text { (Wu dan Pit) }\end{array}$ \\
\hline 21 & $\begin{array}{l}\text { Taiwan } \\
\quad \text { (Republic of China) }\end{array}$ & Taipei City & NA & 25.012 & 121.387 & Bituminous & Small & Shan zi jiao \\
\hline 24 & $\begin{array}{l}\text { Taiwan } \\
\quad \text { (Republic of China) }\end{array}$ & Miaoli City & NA & 24.588 & 120.974 & Bituminous & Small & Ba gua li \\
\hline 29 & $\begin{array}{l}\text { Taiwan } \\
\quad \text { (Republic of China) }\end{array}$ & Miaoli City & NA & 24.533 & 120.998 & Bituminous & Small & Nan shuang \\
\hline 39 & $\begin{array}{l}\text { Taiwan } \\
\quad \text { (Republic of China) }\end{array}$ & Taipei City & NA & 24.956 & 121.418 & Bituminous & Small & $\begin{array}{l}\text { Qing shui keng } \\
\text { (Qing shui Pit) }\end{array}$ \\
\hline 41 & $\begin{array}{l}\text { Taiwan } \\
\quad \text { (Republic of China) }\end{array}$ & Taipei City & NA & 24.978 & 121.551 & Bituminous & Small & Jing mei \\
\hline 43 & $\begin{array}{l}\text { Taiwan } \\
\quad \text { (Republic of China) }\end{array}$ & Taipei City & NA & 24.851 & 121.325 & Bituminous & Medium & San xia Da xi \\
\hline 44 & $\begin{array}{l}\text { Taiwan } \\
\quad \text { (Republic of China) }\end{array}$ & Hsinchu (Xinzhu) City & NA & 24.767 & 121.199 & Bituminous & Small & Guan xi \\
\hline 48 & $\begin{array}{l}\text { Taiwan } \\
\text { (Republic of China) }\end{array}$ & Hsinchu (Xinzhu) City & NA & 24.724 & 121.241 & Bituminous & Small & Jia le \\
\hline 54 & $\begin{array}{l}\text { Taiwan } \\
\text { (Republic of China) }\end{array}$ & Hsinchu (Xinzhu) City & NA & 24.622 & 121.015 & Bituminous & Small & Shi tou shan, Bei pu \\
\hline 20 & Tianjin Municipality & NA & Ji County & 39.752 & 117.496 & Coal & Large & Xia cang \\
\hline 1 & $\begin{array}{l}\text { Xinjiang Uyghur } \\
\text { Autonomous Region }\end{array}$ & Ili Autonomous Prefecture & Yining (Ghulja) County & 44.199 & 80.664 & Bituminous & Large & Lao ai yi tu Well Field \\
\hline 2 & $\begin{array}{l}\text { Xinjiang Uyghur } \\
\text { Autonomous Region }\end{array}$ & Ili Autonomous Prefecture & Yining (Ghulja) County & 44.167 & 81.350 & Bituminous & Medium & Gan gou Survey Area \\
\hline 3 & $\begin{array}{l}\text { Xinjiang Uyghur } \\
\text { Autonomous Region }\end{array}$ & Ili Autonomous Prefecture & $\begin{array}{l}\text { Huocheng (Korgas) } \\
\text { County }\end{array}$ & 43.968 & 80.787 & Bituminous & Small & $\begin{array}{l}\text { Yi ju ga shan } \\
\text { Well Field }\end{array}$ \\
\hline 4 & $\begin{array}{l}\text { Xinjiang Uyghur } \\
\text { Autonomous Region }\end{array}$ & Ili Autonomous Prefecture & $\begin{array}{l}\text { Huocheng (Qorghas) } \\
\text { County }\end{array}$ & 43.930 & 81.053 & Bituminous & Small & No. 1 Well Field \\
\hline 5 & $\begin{array}{l}\text { Xinjiang Uyghur } \\
\text { Autonomous Region }\end{array}$ & Ili Autonomous Prefecture & Yining (Ghulja) County & 43.962 & 81.282 & Bituminous & Small & Survey Area \\
\hline 6 & $\begin{array}{l}\text { Xinjiang Uyghur } \\
\text { Autonomous Region }\end{array}$ & Ili Autonomous Prefecture & Yining (Ghulja) County & 44.040 & 81.563 & Bituminous & Medium & $\begin{array}{l}\text { He dong Area No. } 1 \\
\text { Well Field }\end{array}$ \\
\hline
\end{tabular}


Table 1. List of coal mines in China.-Continued

[Abbreviations used: dec. deg. - decimal degrees; NA - not available or not applicable]

\begin{tabular}{|c|c|c|c|c|c|c|c|c|}
\hline $\begin{array}{c}\text { ID } \\
\text { Number }\end{array}$ & $\begin{array}{c}\text { Province, National } \\
\text { Municipality, or } \\
\text { Autonomous Region }\end{array}$ & $\begin{array}{c}\text { Prefecture, Municipality, } \\
\text { Prefecture-Level City, or } \\
\text { League }\end{array}$ & $\begin{array}{l}\text { County, District or } \\
\text { County-Level City }\end{array}$ & $\begin{array}{l}\text { Latitude } \\
\text { (dec. deg.) }\end{array}$ & $\begin{array}{l}\text { Longitude } \\
\text { (dec. deg.) }\end{array}$ & Rank & $\begin{array}{l}\text { Relative } \\
\text { Mine Size }\end{array}$ & Mine Name \\
\hline 7 & $\begin{array}{l}\text { Xinjiang Uyghur } \\
\text { Autonomous Region }\end{array}$ & Ili Autonomous Prefecture & Yining (Ghulja) County & 44.023 & 81.641 & Bituminous & Small & Ka zan qi Well Field \\
\hline 8 & $\begin{array}{l}\text { Xinjiang Uyghur } \\
\text { Autonomous Region }\end{array}$ & Aletai (Altay) Prefecture & $\begin{array}{l}\text { Jimunai (JeMiney) } \\
\text { County }\end{array}$ & 47.181 & 86.401 & Bituminous & Small & $\begin{array}{l}\text { Ha er jia wu } \\
\text { Well Field }\end{array}$ \\
\hline 9 & $\begin{array}{l}\text { Xinjiang Uyghur } \\
\text { Autonomous Region }\end{array}$ & Tacheng Prefecture & Tuoli (Toli) County & 46.149 & 84.424 & Bituminous & Large & $\begin{array}{l}\text { Tie chang gou } \\
\text { Well Field }\end{array}$ \\
\hline 10 & $\begin{array}{l}\text { Xinjiang Uyghur } \\
\text { Autonomous Region }\end{array}$ & Tacheng Prefecture & $\begin{array}{c}\text { Hebukesa'er (Qobuqsar) } \\
\text { Autonomous County }\end{array}$ & 46.227 & 85.342 & Bituminous & Large & $\begin{array}{l}\text { Tie chang gou - } \\
\text { Bai li shan } \\
\text { Exploration Area }\end{array}$ \\
\hline 11 & $\begin{array}{l}\text { Xinjiang Uyghur } \\
\text { Autonomous Region }\end{array}$ & Tacheng Prefecture & $\begin{array}{c}\text { Hebukesa'er (Qobuqsar) } \\
\text { Autonomous County }\end{array}$ & 46.482 & 85.736 & Bituminous & Small & No. 2 Well Field \\
\hline 12 & $\begin{array}{l}\text { Xinjiang Uyghur } \\
\text { Autonomous Region }\end{array}$ & Tacheng Prefecture & $\begin{array}{c}\text { Hebukesa'er (Qobuqsar) } \\
\text { Autonomous County }\end{array}$ & 46.602 & 85.951 & Bituminous & Medium & No. 1 Well Field \\
\hline 13 & $\begin{array}{l}\text { Xinjiang Uyghur } \\
\text { Autonomous Region }\end{array}$ & Tacheng Prefecture & $\begin{array}{c}\text { Hebukesa'er (Qobuqsar) } \\
\text { Autonomous County }\end{array}$ & 46.396 & 85.960 & Bituminous & Small & No. 3 Well Field \\
\hline 14 & $\begin{array}{l}\text { Xinjiang Uyghur } \\
\text { Autonomous Region }\end{array}$ & Tacheng Prefecture & $\begin{array}{c}\text { Hebukesa'er (Qobuqsar) } \\
\text { Autonomous County }\end{array}$ & 46.391 & 86.371 & Bituminous & Large & $\begin{array}{l}\text { Ku lun tie bu ke } \\
\text { Bai yang he } \\
\text { Survey Area }\end{array}$ \\
\hline 15 & $\begin{array}{l}\text { Xinjiang Uyghur } \\
\text { Autonomous Region }\end{array}$ & Tacheng Prefecture & $\begin{array}{c}\text { Hebukesa'er (Qobuqsar) } \\
\text { Autonomous County }\end{array}$ & 46.583 & 86.432 & Bituminous & Small & No. 4 Well Field \\
\hline 16.1 & $\begin{array}{l}\text { Xinjiang Uyghur } \\
\text { Autonomous Region }\end{array}$ & Tacheng Prefecture & Wusu (Usu) City & 44.169 & 84.211 & Bituminous & Large & Si ke shu Well Field \\
\hline 16.2 & $\begin{array}{l}\text { Xinjiang Uyghur } \\
\text { Autonomous Region }\end{array}$ & Tacheng Prefecture & Wusu (Usu) City & 44.155 & 84.548 & Lignite & Medium & Si ke shu Well Field \\
\hline 17 & $\begin{array}{l}\text { Xinjiang Uyghur } \\
\text { Autonomous Region }\end{array}$ & Tacheng Prefecture & Wusu (Usu) City & 44.024 & 84.960 & Bituminous & Small & \\
\hline 18 & $\begin{array}{l}\text { Xinjiang Uyghur } \\
\text { Autonomous Region }\end{array}$ & Aletai (Altay) Prefecture & $\begin{array}{l}\text { Fuyun (Koktokay) } \\
\text { County }\end{array}$ & 46.547 & 89.278 & Bituminous & Small & Zha he ba Well Field \\
\hline 19 & $\begin{array}{l}\text { Xinjiang Uyghur } \\
\text { Autonomous Region }\end{array}$ & Aletai (Altay) Prefecture & Qinhe (Qinggil) County & 46.346 & 90.378 & Bituminous & Small & Ka la ta si \\
\hline 20 & $\begin{array}{l}\text { Xinjiang Uyghur } \\
\text { Autonomous Region }\end{array}$ & $\begin{array}{l}\text { Changji Autonomous } \\
\text { Prefecture }\end{array}$ & Fukang City & 44.093 & 87.744 & Bituminous & Small & Survey Area 2 \\
\hline 21 & $\begin{array}{l}\text { Xinjiang Uyghur } \\
\text { Autonomous Region }\end{array}$ & $\begin{array}{l}\text { Changji Autonomous } \\
\text { Prefecture }\end{array}$ & Fukang City & 43.910 & 88.289 & Bituminous & Small & $\begin{array}{l}\text { San gong he } \\
\text { Survey Area }\end{array}$ \\
\hline 22 & $\begin{array}{l}\text { Xinjiang Uyghur } \\
\text { Autonomous Region }\end{array}$ & $\begin{array}{l}\text { Changji Autonomous } \\
\text { Prefecture }\end{array}$ & Fukang City & 44.098 & 88.069 & Bituminous & Small & $\begin{array}{l}\text { Xiao huang shan } \\
\text { Survey Area }\end{array}$ \\
\hline 23 & $\begin{array}{l}\text { Xinjiang Uyghur } \\
\text { Autonomous Region }\end{array}$ & $\begin{array}{l}\text { Changji Autonomous } \\
\text { Prefecture }\end{array}$ & Fukang City & 44.080 & 88.201 & Bituminous & Small & $\begin{array}{l}\text { Wu gong he } \\
\text { Well Field }\end{array}$ \\
\hline
\end{tabular}


Table 1. List of coal mines in China.-Continued

[Abbreviations used: dec. deg. - decimal degrees; NA - not available or not applicable]

\begin{tabular}{|c|c|c|c|c|c|c|c|c|}
\hline $\begin{array}{c}\text { ID } \\
\text { Number }\end{array}$ & $\begin{array}{l}\text { Province, National } \\
\text { Municipality, or } \\
\text { Autonomous Region }\end{array}$ & $\begin{array}{c}\text { Prefecture, Municipality, } \\
\text { Prefecture-Level City, or } \\
\text { League }\end{array}$ & $\begin{array}{l}\text { County, District or } \\
\text { County-Level City }\end{array}$ & $\begin{array}{l}\text { Latitude } \\
\text { (dec. deg.) }\end{array}$ & $\begin{array}{l}\text { Longitude } \\
\text { (dec. deg.) }\end{array}$ & Rank & $\begin{array}{l}\text { Relative } \\
\text { Mine Size }\end{array}$ & Mine Name \\
\hline 25 & $\begin{array}{l}\text { Xinjiang Uyghur } \\
\text { Autonomous Region }\end{array}$ & $\begin{array}{l}\text { Changji Autonomous } \\
\text { Prefecture }\end{array}$ & Fukang City & 44.068 & 88.379 & Bituminous & Small & Sha gou Well Field \\
\hline 26 & $\begin{array}{l}\text { Xinjiang Uyghur } \\
\text { Autonomous Region }\end{array}$ & $\begin{array}{l}\text { Changji Autonomous } \\
\text { Prefecture }\end{array}$ & Fukang City & 43.934 & 88.847 & Bituminous & Small & Survey Area \\
\hline 27 & $\begin{array}{l}\text { Xinjiang Uyghur } \\
\text { Autonomous Region }\end{array}$ & $\begin{array}{l}\text { Changji Autonomous } \\
\text { Prefecture }\end{array}$ & Fukang City & 44.037 & 88.607 & Bituminous & Small & $\begin{array}{l}\text { Bai yang he } \\
\text { Well Field }\end{array}$ \\
\hline 28 & $\begin{array}{l}\text { Xinjiang Uyghur } \\
\text { Autonomous Region }\end{array}$ & $\begin{array}{l}\text { Changji Autonomous } \\
\text { Prefecture }\end{array}$ & Qitai (Guchung) County & 44.636 & 89.830 & Bituminous & Small & $\begin{array}{l}\text { Zhun dong Coal } \\
\text { Producing Area }\end{array}$ \\
\hline 29 & $\begin{array}{l}\text { Xinjiang Uyghur } \\
\text { Autonomous Region }\end{array}$ & $\begin{array}{l}\text { Changji Autonomous } \\
\text { Prefecture }\end{array}$ & Qitai (Guchung) County & 44.515 & 90.338 & Bituminous & Small & $\begin{array}{l}\text { Bei shan mei yao } \\
\text { Survey Area }\end{array}$ \\
\hline 30 & $\begin{array}{l}\text { Xinjiang Uyghur } \\
\text { Autonomous Region }\end{array}$ & $\begin{array}{l}\text { Changji Autonomous } \\
\text { Prefecture }\end{array}$ & $\begin{array}{l}\text { Mulei (Mori) } \\
\text { Autonomous County }\end{array}$ & 44.071 & 90.488 & Bituminous & Small & Lao jun miao \\
\hline 31 & $\begin{array}{l}\text { Xinjiang Uyghur } \\
\text { Autonomous Region }\end{array}$ & $\begin{array}{l}\text { Changji Autonomous } \\
\text { Prefecture }\end{array}$ & Qitai County & 44.849 & 91.047 & Bituminous & Small & $\begin{array}{l}\text { Wo tou quan } \\
\text { Survey Area }\end{array}$ \\
\hline 32 & $\begin{array}{l}\text { Xinjiang Uyghur } \\
\text { Autonomous Region }\end{array}$ & Hami Prefecture & $\begin{array}{l}\text { Balikun (Barkol) } \\
\text { Autonomous County }\end{array}$ & 44.456 & 91.848 & Bituminous & Small & Ji lang \\
\hline 33 & $\begin{array}{l}\text { Xinjiang Uyghur } \\
\text { Autonomous Region }\end{array}$ & Hami Prefecture & $\begin{array}{l}\text { Balikun (Barkol) } \\
\text { Autonomous County }\end{array}$ & 44.174 & 92.264 & Bituminous & Small & Shi tan yao Coal Mine \\
\hline 34 & $\begin{array}{l}\text { Xinjiang Uyghur } \\
\text { Autonomous Region }\end{array}$ & Hami Prefecture & $\begin{array}{l}\text { Balikun (Barkol) } \\
\text { Autonomous County }\end{array}$ & 44.291 & 92.345 & Bituminous & Small & Shi tan yao \\
\hline 35 & $\begin{array}{l}\text { Xinjiang Uyghur } \\
\text { Autonomous Region }\end{array}$ & Hami Prefecture & $\begin{array}{l}\text { Balikun (Barkol) } \\
\text { Autonomous County }\end{array}$ & 44.046 & 92.255 & Bituminous & Small & Shi tan yao Coal Mine \\
\hline 36 & $\begin{array}{l}\text { Xinjiang Uyghur } \\
\text { Autonomous Region }\end{array}$ & Hami Prefecture & $\begin{array}{l}\text { Balikun (Barkol) } \\
\text { Autonomous County }\end{array}$ & 44.102 & 92.782 & Bituminous & Small & Shi tan yao Coal Mine \\
\hline 37 & $\begin{array}{l}\text { Xinjiang Uyghur } \\
\text { Autonomous Region }\end{array}$ & Hami Prefecture & $\begin{array}{l}\text { Balikun (Barkol) } \\
\text { Autonomous County }\end{array}$ & 44.393 & 93.249 & Bituminous & Small & San tang hu \\
\hline 38 & $\begin{array}{l}\text { Xinjiang Uyghur } \\
\text { Autonomous Region }\end{array}$ & Ili Autonomous Prefecture & $\begin{array}{l}\text { Zhaosu } \\
\text { (Mongghulkure) } \\
\text { County }\end{array}$ & 43.285 & 81.127 & Bituminous & Small & Hong la hai Well Field \\
\hline 39 & $\begin{array}{l}\text { Xinjiang Uyghur } \\
\text { Autonomous Region }\end{array}$ & Ili Autonomous Prefecture & $\begin{array}{l}\text { Chabucha'er (Qapqal) } \\
\text { Autonomous County }\end{array}$ & 43.547 & 81.650 & Bituminous & Large & Da la di Well Field \\
\hline 40 & $\begin{array}{l}\text { Xinjiang Uyghur } \\
\text { Autonomous Region }\end{array}$ & Ili Autonomous Prefecture & Nileke (Nilka) County & 43.815 & 82.824 & Bituminous & Medium & Ji lun tai Survey Area \\
\hline 41 & $\begin{array}{l}\text { Xinjiang Uyghur } \\
\text { Autonomous Region }\end{array}$ & Ili Autonomous Prefecture & Nileke (Nilka) County & 43.816 & 83.175 & Bituminous & Small & $\begin{array}{l}\text { Sha te bu la ke } \\
\text { Survey Area }\end{array}$ \\
\hline 42 & $\begin{array}{l}\text { Xinjiang Uyghur } \\
\text { Autonomous Region }\end{array}$ & Ili Autonomous Prefecture & Nileke (Nilka) County & 43.773 & 83.235 & Bituminous & Small & Qu er ma Survey Area \\
\hline 43 & $\begin{array}{l}\text { Xinjiang Uyghur } \\
\text { Autonomous Region }\end{array}$ & Akesu (Aksu) Prefecture & Wensu (Onsu) County & 41.710 & 80.176 & Bituminous & Small & $\begin{array}{l}\text { A tuo yi na ke Survey } \\
\text { Area }\end{array}$ \\
\hline
\end{tabular}


Table 1. List of coal mines in China.-Continued

[Abbreviations used: dec. deg. - decimal degrees; NA - not available or not applicable]

\begin{tabular}{|c|c|c|c|c|c|c|c|c|}
\hline $\begin{array}{c}\text { ID } \\
\text { Number }\end{array}$ & $\begin{array}{l}\text { Province, National } \\
\text { Municipality, or } \\
\text { Autonomous Region }\end{array}$ & $\begin{array}{c}\text { Prefecture, Municipality, } \\
\text { Prefecture-Level City, or } \\
\text { League }\end{array}$ & $\begin{array}{l}\text { County, District or } \\
\text { County-Level City }\end{array}$ & $\begin{array}{l}\text { Latitude } \\
\text { (dec. deg.) }\end{array}$ & $\begin{array}{l}\text { Longitude } \\
\text { (dec. deg.) }\end{array}$ & Rank & $\begin{array}{l}\text { Relative } \\
\text { Mine Size }\end{array}$ & Mine Name \\
\hline 44 & $\begin{array}{l}\text { Xinjiang Uyghur } \\
\text { Autonomous Region }\end{array}$ & Akesu (Aksu) Prefecture & Wensu (Onsu) County & 41.780 & 80.693 & Bituminous & Small & $\begin{array}{l}\text { Qiong ku zi ba yi } \\
\text { Survey Area }\end{array}$ \\
\hline 45 & $\begin{array}{l}\text { Xinjiang Uyghur } \\
\text { Autonomous Region }\end{array}$ & Akesu (Aksu) Prefecture & Baicheng (Bay) County & 42.032 & 81.510 & Bituminous & Medium & Tie lie ke Well Field \\
\hline 46 & $\begin{array}{l}\text { Xinjiang Uyghur } \\
\text { Autonomous Region }\end{array}$ & Akesu (Aksu) Prefecture & Baicheng (Bay) County & 42.080 & 81.755 & Bituminous & Small & $\begin{array}{l}\text { Shu shan he Ka la su } \\
\text { Survey Area }\end{array}$ \\
\hline 47 & $\begin{array}{l}\text { Xinjiang Uyghur } \\
\text { Autonomous Region }\end{array}$ & Akesu (Aksu) Prefecture & Baicheng (Bay) County & 42.083 & 81.896 & Bituminous & Medium & Ku er a ken Well Field \\
\hline 48 & $\begin{array}{l}\text { Xinjiang Uyghur } \\
\text { Autonomous Region }\end{array}$ & Akesu (Aksu) Prefecture & Kuche (Kuchar) County & 42.275 & 83.019 & Bituminous & Small & $\begin{array}{l}\text { E huo bu la ke } \\
\text { Well Field }\end{array}$ \\
\hline 49 & $\begin{array}{l}\text { Xinjiang Uyghur } \\
\text { Autonomous Region }\end{array}$ & Akesu (Aksu) Prefecture & Kuche (Kuchar) County & 42.214 & 83.187 & Bituminous & Large & A ai Well Field \\
\hline 50 & $\begin{array}{l}\text { Xinjiang Uyghur } \\
\text { Autonomous Region }\end{array}$ & Akesu (Aksu) Prefecture & Kuche (Kuchar) County & 42.175 & 83.357 & Bituminous & Large & $\begin{array}{l}\text { Bi you le bao ge zi } \\
\text { Well Field }\end{array}$ \\
\hline 51 & $\begin{array}{l}\text { Xinjiang Uyghur } \\
\text { Autonomous Region }\end{array}$ & Shihezi (Shixenze) City & NA & 43.915 & 85.807 & Bituminous & Small & $\begin{array}{l}\text { Shi chang gou } \\
\text { Well Field }\end{array}$ \\
\hline 52 & $\begin{array}{l}\text { Xinjiang Uyghur } \\
\text { Autonomous Region }\end{array}$ & Shihezi (Shixenze) City & NA & 43.909 & 85.986 & Bituminous & Medium & Hong gou Survey Area \\
\hline 53 & $\begin{array}{l}\text { Xinjiang Uyghur } \\
\text { Autonomous Region }\end{array}$ & $\begin{array}{l}\text { Changji Autonomous } \\
\text { Prefecture }\end{array}$ & Manasi (Manas) County & 43.800 & 86.237 & Bituminous & Large & Survey Area \\
\hline 54 & $\begin{array}{l}\text { Xinjiang Uyghur } \\
\text { Autonomous Region }\end{array}$ & Wulumuqi (Urumqi) City & NA & 43.751 & 87.135 & Bituminous & Small & $\begin{array}{l}\text { Liu huang gou } \\
\text { Survey Area }\end{array}$ \\
\hline 55 & $\begin{array}{l}\text { Xinjiang Uyghur } \\
\text { Autonomous Region }\end{array}$ & Wulumuqi (Urumqi ) City & NA & 43.806 & 87.783 & Bituminous & Large & $\begin{array}{l}\text { Liu (6) dao wan } \\
\text { Well Field }\end{array}$ \\
\hline 56 & $\begin{array}{l}\text { Xinjiang Uyghur } \\
\text { Autonomous Region }\end{array}$ & Wulumuqi (Urumqi) City & NA & 43.862 & 87.790 & Bituminous & Medium & $\begin{array}{l}\text { Qi (7) dao wan } \\
\text { Well Field }\end{array}$ \\
\hline 57 & $\begin{array}{l}\text { Xinjiang Uyghur } \\
\text { Autonomous Region }\end{array}$ & Wulumuqi (Urumqi) City & NA & 43.836 & 87.830 & Bituminous & Large & $\begin{array}{l}\mathrm{Ba}(8) \text { dao wan } \\
\text { Well Field }\end{array}$ \\
\hline 58 & $\begin{array}{l}\text { Xinjiang Uyghur } \\
\text { Autonomous Region }\end{array}$ & Wulumuqi (Urumqi) City & NA & 43.886 & 87.835 & Bituminous & Medium & $\begin{array}{l}\text { Jiu (9) dao wan } \\
\text { Well Field }\end{array}$ \\
\hline 59 & $\begin{array}{l}\text { Xinjiang Uyghur } \\
\text { Autonomous Region }\end{array}$ & Wulumuqi (Urumqi) City & NA & 43.863 & 87.866 & Bituminous & Large & Lu cao gou Well Field \\
\hline 60 & $\begin{array}{l}\text { Xinjiang Uyghur } \\
\text { Autonomous Region }\end{array}$ & Wulumuqi (Urumqi ) City & NA & 43.906 & 87.905 & Bituminous & Large & Tie chang gou \\
\hline 61 & $\begin{array}{l}\text { Xinjiang Uyghur } \\
\text { Autonomous Region }\end{array}$ & Wulumuqi (Urumqi) City & NA & 43.900 & 87.935 & Bituminous & Large & $\begin{array}{l}\text { Tie chang gou } \\
\text { Well Field }\end{array}$ \\
\hline 62 & $\begin{array}{l}\text { Xinjiang Uyghur } \\
\text { Autonomous Region }\end{array}$ & Wulumuqi (Urumqi) City & NA & 43.885 & 87.954 & Bituminous & Large & $\begin{array}{l}\text { Wu he Tie chang gou } \\
\text { Survey Area }\end{array}$ \\
\hline
\end{tabular}


Table 1. List of coal mines in China.-Continued

[Abbreviations used: dec. deg. - decimal degrees; NA - not available or not applicable]

\begin{tabular}{|c|c|c|c|c|c|c|c|c|}
\hline $\begin{array}{l}\text { ID } \\
\text { Number }\end{array}$ & $\begin{array}{l}\text { Province, National } \\
\text { Municipality, or } \\
\text { Autonomous Region }\end{array}$ & $\begin{array}{c}\text { Prefecture, Municipality, } \\
\text { Prefecture-Level City, or } \\
\text { League }\end{array}$ & $\begin{array}{l}\text { County, District or } \\
\text { County-Level City }\end{array}$ & $\begin{array}{l}\text { Latitude } \\
\text { (dec. deg.) }\end{array}$ & $\begin{array}{l}\text { Longitude } \\
\text { (dec. deg.) }\end{array}$ & Rank & $\begin{array}{l}\text { Relative } \\
\text { Mine Size }\end{array}$ & Mine Name \\
\hline 63 & $\begin{array}{l}\text { Xinjiang Uyghur } \\
\text { Autonomous Region }\end{array}$ & Wulumuqi (Urumqi ) City & NA & 43.963 & 88.055 & Bituminous & Large & $\begin{array}{l}\text { Wu he Bai yan he } \\
\text { Survey Area }\end{array}$ \\
\hline 64 & $\begin{array}{l}\text { Xinjiang Uyghur } \\
\text { Autonomous Region }\end{array}$ & Wulumuqi (Urumqi ) City & NA & 43.725 & 87.516 & Bituminous & Small & Xi shan Survey Area \\
\hline 65 & $\begin{array}{l}\text { Xinjiang Uyghur } \\
\text { Autonomous Region }\end{array}$ & Wulumuqi (Urumqi ) City & NA & 43.739 & 87.598 & Bituminous & Small & $\begin{array}{l}\text { Si dao pen } \\
\text { Survey Area }\end{array}$ \\
\hline 66 & $\begin{array}{l}\text { Xinjiang Uyghur } \\
\text { Autonomous Region }\end{array}$ & Wulumuqi (Urumqi ) City & NA & 43.731 & 87.616 & Bituminous & Small & $\begin{array}{l}\text { Da fu gou No. } 2 \\
\text { Well Field }\end{array}$ \\
\hline 67 & $\begin{array}{l}\text { Xinjiang Uyghur } \\
\text { Autonomous Region }\end{array}$ & Wulumuqi (Urumqi ) City & NA & 43.711 & 87.615 & Bituminous & Small & $\begin{array}{l}\text { Zhuo zi shan } \\
\text { Survey Area }\end{array}$ \\
\hline 68 & $\begin{array}{l}\text { Xinjiang Uyghur } \\
\text { Autonomous Region }\end{array}$ & Wulumuqi (Urumqi ) City & NA & 43.735 & 87.669 & Bituminous & Small & Da fu gou Well Field \\
\hline 69 & $\begin{array}{l}\text { Xinjiang Uyghur } \\
\text { Autonomous Region }\end{array}$ & Wulumuqi (Urumqi ) City & NA & 43.756 & 87.684 & Bituminous & Small & $\begin{array}{l}\text { Lao jun miao } \\
\text { Survey Area }\end{array}$ \\
\hline 73 & $\begin{array}{l}\text { Xinjiang Uyghur } \\
\text { Autonomous Region }\end{array}$ & Ili Autonomous Prefecture & Nileke (Nilka) County & 43.581 & 84.158 & Bituminous & Small & Ke er ke Survey Area \\
\hline 74 & $\begin{array}{l}\text { Xinjiang Uyghur } \\
\text { Autonomous Region }\end{array}$ & $\begin{array}{l}\text { Bayinguoleng } \\
\text { (Bayin'gholin) } \\
\text { Autonomous Prefecture }\end{array}$ & Hejing County & 43.397 & 85.835 & Bituminous & Small & Gu lun gou \\
\hline 75 & $\begin{array}{l}\text { Xinjiang Uyghur } \\
\text { Autonomous Region }\end{array}$ & Wulumuqi (Urumqi ) City & NA & 43.402 & 87.067 & Bituminous & Large & No. 2 Well Field \\
\hline 76 & $\begin{array}{l}\text { Xinjiang Uyghur } \\
\text { Autonomous Region }\end{array}$ & Wulumuqi (Urumqi ) City & NA & 43.284 & 87.167 & Bituminous & Small & No. 1 Well Field \\
\hline 77 & $\begin{array}{l}\text { Xinjiang Uyghur } \\
\text { Autonomous Region }\end{array}$ & Wulumuqi (Urumqi ) City & NA & 43.266 & 87.226 & Bituminous & Small & No. 3 Well Field \\
\hline 78 & $\begin{array}{l}\text { Xinjiang Uyghur } \\
\text { Autonomous Region }\end{array}$ & Wulumuqi (Urumqi ) City & NA & 43.293 & 87.292 & Bituminous & Small & No. 5 Well Field \\
\hline 79 & $\begin{array}{l}\text { Xinjiang Uyghur } \\
\text { Autonomous Region }\end{array}$ & Wulumuqi (Urumqi ) City & NA & 43.265 & 87.285 & Bituminous & Small & No. 4 Well Field \\
\hline 80 & $\begin{array}{l}\text { Xinjiang Uyghur } \\
\text { Autonomous Region }\end{array}$ & Wulumuqi (Urumqi ) City & NA & 43.244 & 87.553 & Bituminous & Small & Survey Area \\
\hline 81 & $\begin{array}{l}\text { Xinjiang Uyghur } \\
\text { Autonomous Region }\end{array}$ & Wulumuqi (Urumqi ) City & NA & 43.034 & 87.508 & Bituminous & Large & $\begin{array}{l}\text { Ai wei er gou } \\
\text { Well Field }\end{array}$ \\
\hline 82 & $\begin{array}{l}\text { Xinjiang Uyghur } \\
\text { Autonomous Region }\end{array}$ & Wulumuqi (Urumqi ) City & NA & 42.855 & 87.892 & Bituminous & Small & Yu er gou \\
\hline 83 & $\begin{array}{l}\text { Xinjiang Uyghur } \\
\text { Autonomous Region }\end{array}$ & $\begin{array}{l}\text { Bayinguoleng } \\
\text { (Bayingholin) } \\
\text { Autonomous Prefecture }\end{array}$ & Luntai (Bugur) County & 42.124 & 84.529 & Bituminous & Small & Survey Area \\
\hline
\end{tabular}


Table 1. List of coal mines in China.-Continued

[Abbreviations used: dec. deg. - decimal degrees; NA - not available or not applicable]

\begin{tabular}{|c|c|c|c|c|c|c|c|c|}
\hline $\begin{array}{l}\text { ID } \\
\text { Number }\end{array}$ & $\begin{array}{l}\text { Province, National } \\
\text { Municipality, or } \\
\text { Autonomous Region }\end{array}$ & $\begin{array}{l}\text { Prefecture, Municipality, } \\
\text { Prefecture-Level City, or } \\
\text { League }\end{array}$ & $\begin{array}{l}\text { County, District or } \\
\text { County-Level City }\end{array}$ & $\begin{array}{l}\text { Latitude } \\
\text { (dec. deg.) }\end{array}$ & $\begin{array}{l}\text { Longitude } \\
\text { (dec. deg.) }\end{array}$ & Rank & $\begin{array}{l}\text { Relative } \\
\text { Mine Size }\end{array}$ & Mine Name \\
\hline 84 & $\begin{array}{l}\text { Xinjiang Uyghur } \\
\text { Autonomous Region }\end{array}$ & $\begin{array}{l}\text { Bayinguoleng } \\
\text { (Bayingholin) } \\
\text { Autonomous Prefecture }\end{array}$ & Ku'erle (Korla) City & 41.995 & 85.861 & Bituminous & Medium & No. 2 Well Field \\
\hline 85 & $\begin{array}{l}\text { Xinjiang Uyghur } \\
\text { Autonomous Region }\end{array}$ & $\begin{array}{l}\text { Bayinguoleng } \\
\text { (Bayingholin) } \\
\text { Autonomous Prefecture }\end{array}$ & Ku'erle (Korla) City & 41.839 & 85.991 & Bituminous & Medium & Survey Area \\
\hline 86 & $\begin{array}{l}\text { Xinjiang Uyghur } \\
\text { Autonomous Region }\end{array}$ & $\begin{array}{l}\text { Bayinguoleng } \\
\text { (Bayingholin) } \\
\text { Autonomous Prefecture }\end{array}$ & Ku'erle (Korla) City & 41.813 & 86.999 & Bituminous & Small & $\begin{array}{l}\text { Ha man gou } \\
\text { Well Field }\end{array}$ \\
\hline 87 & $\begin{array}{l}\text { Xinjiang Uyghur } \\
\text { Autonomous Region }\end{array}$ & $\begin{array}{l}\text { Bayinguoleng } \\
\text { (Bayingholin) } \\
\text { Autonomous Prefecture }\end{array}$ & Ku'erle (Korla) City & 41.802 & 86.480 & Bituminous & Medium & No. 1 Well Field \\
\hline 88 & $\begin{array}{l}\text { Xinjiang Uyghur } \\
\text { Autonomous Region }\end{array}$ & Wulumuqi (Urumqi) City & NA & 43.511 & 88.489 & Bituminous & Small & Dong gou \\
\hline 89 & $\begin{array}{l}\text { Xinjiang Uyghur } \\
\text { Autonomous Region }\end{array}$ & $\begin{array}{l}\text { Tulufan (Turpan) } \\
\text { Prefecture }\end{array}$ & $\begin{array}{l}\text { Tuokexun (Toqsun) } \\
\text { County }\end{array}$ & 42.970 & 87.884 & Bituminous & Large & $\begin{array}{l}\text { Tu lu bu la ke } \\
\text { Well Field }\end{array}$ \\
\hline 90 & $\begin{array}{l}\text { Xinjiang Uyghur } \\
\text { Autonomous Region }\end{array}$ & $\begin{array}{l}\text { Tulufan (Turpan) } \\
\text { Prefecture }\end{array}$ & $\begin{array}{l}\text { Tuokexun (Toqsun) } \\
\text { County }\end{array}$ & 43.237 & 88.120 & Bituminous & Large & Survey Area \\
\hline 91 & $\begin{array}{l}\text { Xinjiang Uyghur } \\
\text { Autonomous Region }\end{array}$ & $\begin{array}{l}\text { Tulufan (Turpan) } \\
\text { Prefecture }\end{array}$ & $\begin{array}{l}\text { Tuokexun (Toqsun) } \\
\text { County }\end{array}$ & 43.085 & 88.196 & Bituminous & Medium & No. 1 Well Field \\
\hline 92 & $\begin{array}{l}\text { Xinjiang Uyghur } \\
\text { Autonomous Region }\end{array}$ & $\begin{array}{l}\text { Tulufan (Turpan) } \\
\text { Prefecture }\end{array}$ & $\begin{array}{l}\text { Tuokexun (Toqsun) } \\
\text { County }\end{array}$ & 43.102 & 88.234 & Bituminous & Large & No. 4 Well Field \\
\hline 93 & $\begin{array}{l}\text { Xinjiang Uyghur } \\
\text { Autonomous Region }\end{array}$ & $\begin{array}{l}\text { Tulufan (Turpan) } \\
\text { Prefecture }\end{array}$ & NA & 43.269 & 89.310 & Bituminous & Small & Survey Area \\
\hline 94 & $\begin{array}{l}\text { Xinjiang Uyghur } \\
\text { Autonomous Region }\end{array}$ & $\begin{array}{l}\text { Tulufan (Turpan) } \\
\text { Prefecture }\end{array}$ & NA & 43.264 & 89.561 & Bituminous & Small & $\begin{array}{l}\text { Hong hui cao } \\
\text { Well Field }\end{array}$ \\
\hline 95 & $\begin{array}{l}\text { Xinjiang Uyghur } \\
\text { Autonomous Region }\end{array}$ & $\begin{array}{l}\text { Tulufan (Turpan) } \\
\text { Prefecture }\end{array}$ & $\begin{array}{l}\text { Shanshan (Piqan) } \\
\text { County }\end{array}$ & 43.208 & 90.136 & Bituminous & Small & $\begin{array}{c}\text { Ke ke ya (East) } \\
\text { Survey Area }\end{array}$ \\
\hline 96.1 & $\begin{array}{l}\text { Xinjiang Uyghur } \\
\text { Autonomous Region }\end{array}$ & $\begin{array}{l}\text { Tulufan (Turpan) } \\
\text { Prefecture }\end{array}$ & NA & 42.782 & 89.484 & Bituminous & Large & $\begin{array}{l}\text { Ai ding Lake } \\
\text { Survey Area }\end{array}$ \\
\hline 96.2 & $\begin{array}{l}\text { Xinjiang Uyghur } \\
\text { Autonomous Region }\end{array}$ & $\begin{array}{l}\text { Tulufan (Turpan) } \\
\text { Prefecture }\end{array}$ & NA & 42.683 & 90.079 & Lignite & Large & $\begin{array}{l}\text { Ai ding Lake } \\
\text { Survey Area }\end{array}$ \\
\hline 97 & $\begin{array}{l}\text { Xinjiang Uyghur } \\
\text { Autonomous Region }\end{array}$ & $\begin{array}{l}\text { Tulufan (Turpan) } \\
\text { Prefecture }\end{array}$ & $\begin{array}{l}\text { Tuokexun (Toqsun) } \\
\text { County }\end{array}$ & 41.805 & 88.783 & Bituminous & Small & $\begin{array}{l}\text { Suke Suke } \\
\text { Survey Area }\end{array}$ \\
\hline 98 & $\begin{array}{l}\text { Xinjiang Uyghur } \\
\text { Autonomous Region }\end{array}$ & Hami Prefecture & NA & 42.454 & 91.631 & Bituminous & Large & $\begin{array}{l}\text { Sha er Lake } \\
\text { Survey Area }\end{array}$ \\
\hline 99 & $\begin{array}{l}\text { Xinjiang Uyghur } \\
\text { Autonomous Region }\end{array}$ & Hami Prefecture & NA & 43.241 & 92.435 & Bituminous & Medium & $\begin{array}{l}\text { San dao ling Coal } \\
\text { Mine, Sha dun zi } \\
\text { Survey Area }\end{array}$ \\
\hline
\end{tabular}


Table 1. List of coal mines in China.-Continued

[Abbreviations used: dec. deg. - decimal degrees; NA - not available or not applicable]

\begin{tabular}{|c|c|c|c|c|c|c|c|c|}
\hline $\begin{array}{c}\text { ID } \\
\text { Number }\end{array}$ & $\begin{array}{c}\text { Province, National } \\
\text { Municipality, or } \\
\text { Autonomous Region }\end{array}$ & $\begin{array}{c}\text { Prefecture, Municipality, } \\
\text { Prefecture-Level City, or } \\
\text { League }\end{array}$ & $\begin{array}{l}\text { County, District or } \\
\text { County-Level City }\end{array}$ & $\begin{array}{l}\text { Latitude } \\
\text { (dec. deg.) }\end{array}$ & $\begin{array}{l}\text { Longitude } \\
\text { (dec. deg.) }\end{array}$ & Rank & $\begin{array}{l}\text { Relative } \\
\text { Mine Size }\end{array}$ & Mine Name \\
\hline 100 & $\begin{array}{l}\text { Xinjiang Uyghur } \\
\text { Autonomous Region }\end{array}$ & Hami Prefecture & NA & 43.134 & 92.385 & Bituminous & Small & $\begin{array}{l}\text { San dao ling Coal } \\
\text { Mine, Hou yao } \\
\text { Well Field }\end{array}$ \\
\hline 101 & $\begin{array}{l}\text { Xinjiang Uyghur } \\
\text { Autonomous Region }\end{array}$ & Hami Prefecture & NA & 43.036 & 92.420 & Bituminous & Small & $\begin{array}{l}\text { San dao ling } \\
\text { Coal Mine, } \\
\text { Xiao huang shan }\end{array}$ \\
\hline 102 & $\begin{array}{l}\text { Xinjiang Uyghur } \\
\text { Autonomous Region }\end{array}$ & Hami Prefecture & NA & 43.050 & 92.691 & Bituminous & Small & $\begin{array}{l}\text { San dao ling Coal } \\
\text { Mine, Xi shan } \\
\text { Survey Area }\end{array}$ \\
\hline 103 & $\begin{array}{l}\text { Xinjiang Uyghur } \\
\text { Autonomous Region }\end{array}$ & Hami Prefecture & NA & 43.050 & 93.013 & Bituminous & Large & $\begin{array}{l}\text { San dao ling Coal } \\
\text { Mine, Bei quan } \\
\text { Well Field }\end{array}$ \\
\hline 104 & $\begin{array}{l}\text { Xinjiang Uyghur } \\
\text { Autonomous Region }\end{array}$ & Hami Prefecture & NA & 43.282 & 92.727 & Bituminous & Small & $\begin{array}{l}\text { San dao ling Coal } \\
\text { Mine, No. 1 Xie } \\
\text { jing Well Field }\end{array}$ \\
\hline 105 & $\begin{array}{l}\text { Xinjiang Uyghur } \\
\text { Autonomous Region }\end{array}$ & Hami Prefecture & NA & 43.248 & 92.980 & Bituminous & Medium & $\begin{array}{l}\text { San dao ling Coal } \\
\text { Mine, Sha zao quan }\end{array}$ \\
\hline 106 & $\begin{array}{l}\text { Xinjiang Uyghur } \\
\text { Autonomous Region }\end{array}$ & Hami Prefecture & NA & 42.319 & 93.035 & Bituminous & Large & $\begin{array}{l}\text { Da nan hu Coal Mine, } \\
\text { Survey Area }\end{array}$ \\
\hline 107 & $\begin{array}{l}\text { Xinjiang Uyghur } \\
\text { Autonomous Region }\end{array}$ & Hami Prefecture & NA & 42.417 & 95.550 & Bituminous & Medium & $\begin{array}{l}\text { Ye ma quan Coal } \\
\text { Mine, No. } 1 \\
\text { Well Field }\end{array}$ \\
\hline 108 & $\begin{array}{l}\text { Xinjiang Uyghur } \\
\text { Autonomous Region }\end{array}$ & $\begin{array}{l}\text { Kezilesu (Krzilsu) } \\
\text { Autonomous Prefecture }\end{array}$ & $\begin{array}{l}\text { Wuqia (Ulughchat) } \\
\text { County }\end{array}$ & 39.993 & 74.553 & Bituminous & Small & Sha li bai Field \\
\hline 109 & $\begin{array}{l}\text { Xinjiang Uyghur } \\
\text { Autonomous Region }\end{array}$ & $\begin{array}{l}\text { Kezilesu (Krzilsu) } \\
\text { Autonomous Prefecture }\end{array}$ & $\begin{array}{l}\text { Wuqia (Ulughchat) } \\
\text { County }\end{array}$ & 39.785 & 75.038 & Bituminous & Small & Kang su Well Field \\
\hline 110 & $\begin{array}{l}\text { Xinjiang Uyghur } \\
\text { Autonomous Region }\end{array}$ & $\begin{array}{l}\text { Kezilesu (Krzilsu) } \\
\text { Autonomous Prefecture }\end{array}$ & $\begin{array}{l}\text { Wuqia (Ulughchat) } \\
\text { County }\end{array}$ & 39.746 & 75.093 & Bituminous & Small & \\
\hline 111.1 & $\begin{array}{l}\text { Xinjiang Uyghur } \\
\text { Autonomous Region }\end{array}$ & $\begin{array}{l}\text { Kezilesu (Krzilsu) } \\
\text { Autonomous Prefecture }\end{array}$ & Aketao (Akto) County & 38.420 & 76.045 & Bituminous & Small & Sha se di ke \\
\hline 111.2 & $\begin{array}{l}\text { Xinjiang Uyghur } \\
\text { Autonomous Region }\end{array}$ & $\begin{array}{l}\text { Kezilesu (Krzilsu) } \\
\text { Autonomous Prefecture }\end{array}$ & NA & 38.249 & 75.950 & Bituminous & Small & Sha se di ke \\
\hline 112 & $\begin{array}{l}\text { Xinjiang Uyghur } \\
\text { Autonomous Region }\end{array}$ & $\begin{array}{l}\text { Kezilesu (Krzilsu) } \\
\text { Autonomous Prefecture }\end{array}$ & Aketao (Akto) County & 38.046 & 76.331 & Bituminous & Small & $\mathrm{Ku}$ si la fu \\
\hline 113 & $\begin{array}{l}\text { Xinjiang Uyghur } \\
\text { Autonomous Region }\end{array}$ & Kashi (Kashgar) Prefecture & Shache (Yeken) County & 37.726 & 76.692 & Bituminous & Small & $\begin{array}{l}\text { Ge liu mu Coal Mine, } \\
\text { Ke la tu si } \\
\text { Well Field }\end{array}$ \\
\hline 114 & $\begin{array}{l}\text { Xinjiang Uyghur } \\
\text { Autonomous Region }\end{array}$ & Hetian (Hotan) Prefecture & Pishan (Guma) County & 37.061 & 78.886 & Bituminous & Small & Du wa Well Field \\
\hline
\end{tabular}


Table 1. List of coal mines in China.-Continued

[Abbreviations used: dec. deg. - decimal degrees; NA - not available or not applicable]

\begin{tabular}{|c|c|c|c|c|c|c|c|c|}
\hline $\begin{array}{c}\text { ID } \\
\text { Number }\end{array}$ & $\begin{array}{l}\text { Province, National } \\
\text { Municipality, or } \\
\text { Autonomous Region }\end{array}$ & $\begin{array}{c}\text { Prefecture, Municipality, } \\
\text { Prefecture-Level City, or } \\
\text { League }\end{array}$ & $\begin{array}{l}\text { County, District or } \\
\text { County-Level City }\end{array}$ & $\begin{array}{l}\text { Latitude } \\
\text { (dec. deg.) }\end{array}$ & $\begin{array}{l}\text { Longitude } \\
\text { (dec. deg.) }\end{array}$ & Rank & $\begin{array}{l}\text { Relative } \\
\text { Mine Size }\end{array}$ & Mine Name \\
\hline 115 & $\begin{array}{l}\text { Xinjiang Uyghur } \\
\text { Autonomous Region }\end{array}$ & Hetian (Hotan) Prefecture & NA & 36.365 & 79.990 & Bituminous & Small & Bu ya Well Field \\
\hline 116 & $\begin{array}{l}\text { Xinjiang Uyghur } \\
\text { Autonomous Region }\end{array}$ & $\begin{array}{l}\text { Bayinguoleng } \\
\text { (Bayingholin) } \\
\text { Autonomous Prefecture }\end{array}$ & $\begin{array}{l}\text { Ruoqian (Chaqiliq) } \\
\text { County }\end{array}$ & 38.166 & 88.888 & Bituminous & Small & Gai xi \\
\hline 32 & $\begin{array}{l}\text { Xizang (Tibet) } \\
\text { Autonomous Region }\end{array}$ & Nagchu Prefecture & Anduo (Amdo) County & 32.925 & 91.137 & Anthracite & Small & Ga er gen \\
\hline 33 & $\begin{array}{l}\text { Xizang (Tibet) } \\
\text { Autonomous Region }\end{array}$ & Nagchu Prefecture & Anduo (Amdo) County & 32.842 & 91.349 & Anthracite & Small & Tu men ge la \\
\hline 35 & $\begin{array}{l}\text { Xizang (Tibet) } \\
\text { Autonomous Region }\end{array}$ & Chamdo Prefecture & $\begin{array}{l}\text { Dingqing (Dengqen) } \\
\text { County }\end{array}$ & 32.293 & 94.897 & Anthracite & Small & $\mathrm{Zi} \mathrm{jia} \mathrm{pu}$ \\
\hline 36 & $\begin{array}{l}\text { Xizang (Tibet) } \\
\text { Autonomous Region }\end{array}$ & Ngari Prefecture & Ga'er (Gar) County & 31.370 & 80.889 & Bituminous & Small & \\
\hline 54 & $\begin{array}{l}\text { Xizang (Tibet) } \\
\text { Autonomous Region }\end{array}$ & Naqu Prefecture & Naqu County & 31.386 & 92.944 & Lignite & Small & Se xiong \\
\hline 59 & $\begin{array}{l}\text { Xizang (Tibet) } \\
\text { Autonomous Region }\end{array}$ & Chamdo Prefecture & $\begin{array}{l}\text { Leiwuqi (Riwoqe ) } \\
\text { County }\end{array}$ & 31.425 & 96.754 & Bituminous & Small & Ren qing \\
\hline 60 & $\begin{array}{l}\text { Xizang (Tibet) } \\
\text { Autonomous Region }\end{array}$ & Chamdo Prefecture & $\begin{array}{l}\text { Leiwuqi (Riwoqe) } \\
\text { County }\end{array}$ & 31.397 & 96.693 & Anthracite & Small & Ma cha la \\
\hline 61 & $\begin{array}{l}\text { Xizang (Tibet) } \\
\text { Autonomous Region }\end{array}$ & Chamdo Prefecture & $\begin{array}{l}\text { Gongjue (Gonjo) } \\
\text { County }\end{array}$ & 31.195 & 97.881 & Bituminous & Small & Duo gai la \\
\hline 1 & Yunnan Province & $\begin{array}{l}\text { Dali Autonomous } \\
\text { Prefecture }\end{array}$ & Jianchuan County & 26.589 & 99.833 & Lignite & Small & Shuang he \\
\hline 2 & Yunnan Province & Lijiang City & Huaping County & 26.733 & 101.107 & Bituminous & Small & A niu ping \\
\hline 4 & Yunnan Province & Lijiang City & NA & 26.693 & 100.061 & Lignite & Medium & Ru nan \\
\hline 5 & Yunnan Province & Lijiang City & Huaping County & 26.517 & 101.319 & Bituminous & Medium & La shi gou \\
\hline 6 & Yunnan Province & $\begin{array}{l}\text { Dali Autonomous } \\
\text { Prefecture }\end{array}$ & Heqing County & 26.470 & 100.061 & Anthracite & Medium & Hei ni shao ma chang \\
\hline 7 & Yunnan Province & $\begin{array}{l}\text { Dali Autonomous } \\
\text { Prefecture }\end{array}$ & Eryuan County & 26.289 & 100.037 & Lignite & Medium & San ying Tai ping cun \\
\hline 8 & Yunnan Province & Zhaotong City & NA & 27.336 & 103.752 & Lignite & Large & Dong nan jiao \\
\hline 9 & Yunnan Province & Zhaotong City & NA & 27.200 & 103.636 & Lignite & Medium & Hong wei \\
\hline 10 & Yunnan Province & Zhaotong City & Yanjin County & 27.901 & 104.392 & Anthracite & Medium & Miao ba \\
\hline 11 & Yunnan Province & Zhaotong City & Weixin County & 27.916 & 104.983 & Bituminous & Medium & Xin zhuang \\
\hline 12 & Yunnan Province & Zhaotong City & Zhenxiong County & 27.781 & 105.034 & Bituminous & Medium & Wei xin Shi kan \\
\hline 13 & Yunnan Province & Zhaotong City & Zhenxiong County & 27.746 & 104.840 & Anthracite & Medium & Wei xin Ma he \\
\hline 14 & Yunnan Province & Zhaotong City & Yiliang County & 27.756 & 104.333 & Anthracite & Medium & Luo wang \\
\hline 15 & Yunnan Province & Zhaotong City & Zhenxiong County & 27.640 & 105.238 & Anthracite & Medium & Ze di \\
\hline 16 & Yunnan Province & Zhaotong City & Zhenxiong County & 27.599 & 105.178 & Anthracite & Medium & Mu xiang \\
\hline
\end{tabular}


Table 1. List of coal mines in China. - Continued

[Abbreviations used: dec. deg. - decimal degrees; NA - not available or not applicable]

\begin{tabular}{|c|c|c|c|c|c|c|c|c|}
\hline $\begin{array}{c}\text { ID } \\
\text { Number }\end{array}$ & $\begin{array}{c}\text { Province, National } \\
\text { Municipality, or } \\
\text { Autonomous Region }\end{array}$ & $\begin{array}{c}\text { Prefecture, Municipality, } \\
\text { Prefecture-Level City, or } \\
\text { League }\end{array}$ & $\begin{array}{l}\text { County, District or } \\
\text { County-Level City }\end{array}$ & $\begin{array}{c}\text { Latitude } \\
\text { (dec. deg.) }\end{array}$ & $\begin{array}{l}\text { Longitude } \\
\text { (dec. deg.) }\end{array}$ & Rank & $\begin{array}{l}\text { Relative } \\
\text { Mine Size }\end{array}$ & Mine Name \\
\hline 17 & Yunnan Province & Zhaotong City & Zhenxiong County & 27.555 & 105.005 & Anthracite & Medium & \\
\hline 18 & Yunnan Province & Zhaotong City & Zhenxiong County & 27.491 & 104.695 & Anthracite & Medium & Niu chang Bo ji wan \\
\hline 19 & Yunnan Province & Zhaotong City & Zhenxiong County & 27.391 & 104.869 & Anthracite & Medium & Qing shan \\
\hline 20 & Yunnan Province & Qujing City & Xuanwei City & 26.591 & 104.250 & Bituminous & Medium & Tang tang \\
\hline 21 & Yunnan Province & Qujing City & Xuanwei City & 26.541 & 104.507 & Bituminous & Medium & Gu xin \\
\hline 22 & Yunnan Province & Qujing City & Xuanwei City & 26.374 & 104.328 & Bituminous & Large & A zhi \\
\hline 23 & Yunnan Province & Qujing City & Xuanwei City & 26.370 & 104.491 & Bituminous & Large & Bao shan \\
\hline 24 & Yunnan Province & Qujing City & Xuanwei City & 26.315 & 104.252 & Bituminous & Medium & Lai bin pu Chang po \\
\hline 25 & Yunnan Province & Qujing City & Xuanwei City & 26.009 & 104.243 & Bituminous & Medium & Yang chang \\
\hline 26 & Yunnan Province & Qujing City & Xuanwei City & 26.018 & 104.379 & Bituminous & Large & De lai \\
\hline 27 & Yunnan Province & Baoshan City & Xuanwei City & 25.197 & 99.252 & Lignite & Small & Luo zhai \\
\hline 28 & Yunnan Province & Baoshan City & Xuanwei City & 25.109 & 99.185 & Lignite & Medium & Cheng jiao \\
\hline 30 & Yunnan Province & Baoshan City & Xuanwei City & 24.950 & 99.256 & Lignite & Small & Yang yi \\
\hline 31 & Yunnan Province & Baoshan City & Changning County & 24.816 & 99.630 & Lignite & Medium & Hong xing \\
\hline 32 & Yunnan Province & Baoshan City & Longling County & 24.682 & 98.841 & Lignite & Medium & Zhe nan \\
\hline 34 & Yunnan Province & Baoshan City & Changning County & 24.533 & 99.316 & Lignite & Small & Wan dian \\
\hline 35 & Yunnan Province & Lincang City & Yongde County & 24.016 & 99.254 & Lignite & Small & $\mathrm{Hu}$ nai \\
\hline 36 & Yunnan Province & $\begin{array}{l}\text { Chuxiong Autonomous } \\
\text { Prefecture }\end{array}$ & Yao'an County & 25.536 & 101.230 & Lignite & Large & \\
\hline 38 & Yunnan Province & $\begin{array}{l}\text { Dali Autonomous } \\
\text { Prefecture }\end{array}$ & Xiangyun County & 25.466 & 100.732 & Lignite & Small & Yun nan yi \\
\hline 39 & Yunnan Province & $\begin{array}{l}\text { Dali Autonomous } \\
\text { Prefecture }\end{array}$ & Midu County & 25.191 & 100.508 & Lignite & Small & Mi zhi \\
\hline 40 & Yunnan Province & $\begin{array}{l}\text { Chuxiong Autonomous } \\
\text { Prefecture }\end{array}$ & NA & 25.160 & 101.304 & Lignite & Medium & Lu he \\
\hline 41 & Yunnan Province & $\begin{array}{l}\text { Chuxiong Autonomous } \\
\text { Prefecture }\end{array}$ & Lufeng County & 25.075 & 101.916 & Bituminous & Small & Ping lang \\
\hline 42 & Yunnan Province & Pu'er City & $\begin{array}{l}\text { Zhenyuan Autonomous } \\
\text { County }\end{array}$ & 24.073 & 101.258 & Lignite & Medium & San zhang tian \\
\hline 43 & Yunnan Province & Kunming City & $\begin{array}{l}\text { Xundian Autonomous } \\
\text { County }\end{array}$ & 25.554 & 103.197 & Lignite & Small & Jin suo \\
\hline 44 & Yunnan Province & Qujing City & NA & 25.524 & 103.745 & Lignite & Medium & Ma huang chong \\
\hline 45 & Yunnan Province & Kunming City & $\begin{array}{l}\text { Xundian Autonomous } \\
\text { County }\end{array}$ & 25.504 & 102.866 & Lignite & Small & Ke lang \\
\hline 46 & Yunnan Province & Kunming City & $\begin{array}{l}\text { Xundian Autonomous } \\
\text { County }\end{array}$ & 25.443 & 103.117 & Lignite & Small & Xian feng \\
\hline 47 & Yunnan Province & $\begin{array}{l}\text { Chuxiong Autonomous } \\
\text { Prefecture }\end{array}$ & Lufeng County & 25.402 & 102.286 & Lignite & Large & Luo ci Da zhu jie \\
\hline 48 & Yunnan Province & Qujing City & NA & 25.321 & 103.879 & Lignite & Large & Yue zhou \\
\hline
\end{tabular}


Table 1. List of coal mines in China. - Continued

[Abbreviations used: dec. deg. - decimal degrees; NA - not available or not applicable]

\begin{tabular}{|c|c|c|c|c|c|c|c|c|}
\hline $\begin{array}{c}\text { ID } \\
\text { Number }\end{array}$ & $\begin{array}{l}\text { Province, National } \\
\text { Municipality, or } \\
\text { Autonomous Region }\end{array}$ & $\begin{array}{c}\text { Prefecture, Municipality, } \\
\text { Prefecture-Level City, or } \\
\text { League }\end{array}$ & $\begin{array}{l}\text { County, District or } \\
\text { County-Level City }\end{array}$ & $\begin{array}{l}\text { Latitude } \\
\text { (dec. deg.) }\end{array}$ & $\begin{array}{l}\text { Longitude } \\
\text { (dec. deg.) }\end{array}$ & Rank & $\begin{array}{l}\text { Relative } \\
\text { Mine Size }\end{array}$ & Mine Name \\
\hline 49 & Yunnan Province & $\begin{array}{l}\text { Chuxiong Autonomous } \\
\text { Prefecture }\end{array}$ & Lufeng County & 25.259 & 102.302 & Lignite & Small & Luo ci hot spring \\
\hline 50 & Yunnan Province & Kunming City & Songming County & 25.224 & 103.080 & Lignite & Small & Yang lin \\
\hline 51 & Yunnan Province & Kunming City & Yiliang County & 25.195 & 103.214 & Lignite & Medium & Ma jie \\
\hline 52 & Yunnan Province & Kunming City & NA & 25.103 & 102.749 & Lignite & Small & Song hua \\
\hline 53 & Yunnan Province & Kunming City & Yiliang County & 25.002 & 103.042 & Lignite & Large & Ke bao Feng ming cun \\
\hline 56 & Yunnan Province & Qujing City & Shizong County & 24.871 & 103.819 & Bituminous & Small & Ya zi tang \\
\hline 57 & Yunnan Province & Qujing City & Shizong County & 24.740 & 103.688 & Bituminous & Small & Bai zi gou \\
\hline 58 & Yunnan Province & Yuxi City & $\begin{array}{l}\text { Xinping Autonomous } \\
\text { County }\end{array}$ & 24.639 & 103.606 & Bituminous & Small & Gui shan \\
\hline 59 & Yunnan Province & $\begin{array}{l}\text { Honghe Autonomous } \\
\text { Prefecture }\end{array}$ & Mile City & 24.535 & 103.498 & Bituminous & Small & Jiao luo zhao \\
\hline 60 & Yunnan Province & Yuxi City & $\begin{array}{l}\text { Hongta District, } \\
\text { Chunhe Township }\end{array}$ & 24.376 & 102.492 & Lignite & Small & Guo jing \\
\hline 61 & Yunnan Province & Yuxi City & NA & 24.368 & 102.557 & Lignite & Small & You sou \\
\hline 62 & Yunnan Province & Yuxi City & Jianchuan County & 24.319 & 102.730 & Lignite & Small & Shang tou ying \\
\hline 63 & Yunnan Province & Yuxi City & $\begin{array}{l}\text { Eshan Autonomous } \\
\text { County }\end{array}$ & 24.271 & 102.123 & Anthracite & Small & Ta dian \\
\hline 64 & Yunnan Province & Yuxi City & Huaning County & 24.225 & 102.953 & Lignite & Medium & Cheng jiao \\
\hline 65 & Yunnan Province & $\begin{array}{l}\text { Honghe Autonomous } \\
\text { Prefecture }\end{array}$ & Mile City & 24.249 & 103.458 & Lignite & Medium & Kua zhu \\
\hline 66 & Yunnan Province & Qujing City & NA & 25.938 & 104.218 & Bituminous & Medium & $\mathrm{Ka} \mathrm{ju}$ \\
\hline 67 & Yunnan Province & Qujing City & Fuyuan County & 25.885 & 104.342 & Bituminous & Small & Qing yun \\
\hline 68 & Yunnan Province & Qujing City & Fuyuan County & 25.836 & 104.304 & Bituminous & Small & Hou suo \\
\hline 69 & Yunnan Province & Qujing City & NA & 25.821 & 104.123 & Lignite & Medium & Zhan yi luo shui \\
\hline 70 & Yunnan Province & Qujing City & Fuyuan County & 25.592 & 104.234 & Bituminous & Small & Long hai gou \\
\hline 71 & Yunnan Province & Qujing City & Fuyuan County & 25.440 & 104.339 & Bituminous & Small & Yun shan \\
\hline 72 & Yunnan Province & Qujing City & Fuyuan County & 25.365 & 104.407 & Bituminous & Medium & Fu cun \\
\hline 73 & Yunnan Province & Qujing City & Fuyuan County & 25.328 & 104.552 & Bituminous & Medium & $\mathrm{Gu} \mathrm{mu}$ \\
\hline 74 & Yunnan Province & Qujing City & Fuyuan County & 25.317 & 104.226 & Bituminous & Large & Qu jing En hong \\
\hline 75 & Yunnan Province & Qujing City & Fuyuan County & 25.213 & 104.534 & Anthracite & Large & Lao chang \\
\hline 76 & Yunnan Province & Qujing City & Luoping County & 25.034 & 104.101 & Anthracite & Small & A gang Qing pin shao \\
\hline 78 & Yunnan Province & Pu'er City & $\begin{array}{l}\text { Lancang Autonomous } \\
\text { County }\end{array}$ & 23.037 & 99.795 & Lignite & Small & $\begin{array}{l}\text { Shang yun Mang jiao } \\
\text { Man yang }\end{array}$ \\
\hline 79 & Yunnan Province & Pu'er City & $\begin{array}{l}\text { Lancang Autonomous } \\
\text { County }\end{array}$ & 22.504 & 99.902 & Lignite & Medium & ??? bin \\
\hline 80 & Yunnan Province & Lincang City & NA & 23.917 & 100.030 & Lignite & Small & ??? wang \\
\hline 81 & Yunnan Province & $\begin{array}{l}\text { Xishuangbann Autonomous } \\
\text { Prefecture }\end{array}$ & Jinghong City & 22.087 & 100.852 & Lignite & Small & Xiao ??? Yang \\
\hline
\end{tabular}


Table 1. List of coal mines in China.-Continued

[Abbreviations used: dec. deg. - decimal degrees; NA - not available or not applicable]

\begin{tabular}{|c|c|c|c|c|c|c|c|c|}
\hline $\begin{array}{c}\text { ID } \\
\text { Number }\end{array}$ & $\begin{array}{c}\text { Province, National } \\
\text { Municipality, or } \\
\text { Autonomous Region }\end{array}$ & $\begin{array}{c}\text { Prefecture, Municipality, } \\
\text { Prefecture-Level City, or } \\
\text { League }\end{array}$ & $\begin{array}{l}\text { County, District or } \\
\text { County-Level City }\end{array}$ & $\begin{array}{l}\text { Latitude } \\
\text { (dec. deg.) }\end{array}$ & $\begin{array}{l}\text { Longitude } \\
\text { (dec. deg.) }\end{array}$ & Rank & $\begin{array}{l}\text { Relative } \\
\text { Mine Size }\end{array}$ & Mine Name \\
\hline 82 & Yunnan Province & $\begin{array}{l}\text { Honghe Autonomous } \\
\text { Prefecture }\end{array}$ & Kaiyuan City & 23.811 & 103.213 & Lignite & Medium & Xiao long tan \\
\hline 83 & Yunnan Province & $\begin{array}{l}\text { Honghe Autonomous } \\
\text { Prefecture }\end{array}$ & Shiping County & 23.763 & 102.403 & Lignite & Small & Bao xiu \\
\hline 84 & Yunnan Province & $\begin{array}{l}\text { Honghe Autonomous } \\
\text { Prefecture }\end{array}$ & Jianshui City & 23.631 & 102.805 & Lignite & Small & Cheng jiao \\
\hline 85 & Yunnan Province & $\begin{array}{l}\text { Honghe Autonomous } \\
\text { Prefecture }\end{array}$ & Jianshui City & 23.653 & 102.990 & Lignite & Small & Yang tian \\
\hline 86 & Yunnan Province & $\begin{array}{l}\text { Honghe Autonomous } \\
\text { Prefecture }\end{array}$ & Shiping County & 23.452 & 102.445 & Anthracite & Small & Niu jie \\
\hline 87 & Yunnan Province & $\begin{array}{l}\text { Honghe Autonomous } \\
\text { Prefecture }\end{array}$ & Mengzi City & 23.473 & 103.354 & Lignite & Medium & Yu guo pu \\
\hline 88 & Yunnan Province & $\begin{array}{l}\text { Wenshan Autonomous } \\
\text { Prefecture }\end{array}$ & Funing County & 23.498 & 105.231 & Lignite & Medium & Pu yang \\
\hline 1 & Zhejiang Province & Huzhou City & Changxing County & 31.132 & 119.751 & Bituminous & Medium & Mei shan \\
\hline 2 & Zhejiang Province & Huzhou City & Changxing County & 30.969 & 119.943 & Bituminous & Small & Li jia xiang \\
\hline 3 & Zhejiang Province & Huzhou City & NA & 30.944 & 119.966 & Anthracite & Small & Huang zhi shan \\
\hline 8 & Zhejiang Province & Huzhou City & NA & 30.987 & 120.011 & Bituminous & Small & Heng shan qiao \\
\hline 9 & Zhejiang Province & Huzhou City & NA & 30.955 & 120.077 & Anthracite & Small & Nan gao qiao \\
\hline 11 & Zhejiang Province & Huzhou City & NA & 30.914 & 120.013 & Anthracite & Small & Gan shan \\
\hline 12 & Zhejiang Province & Huzhou City & NA & 30.890 & 120.055 & Anthracite & Small & Yang jia bu \\
\hline 20 & Zhejiang Province & Hangzhou City & Tonglu County & 29.920 & 119.540 & Anthracite & Small & Zhi nan \\
\hline 24 & Zhejiang Province & Hangzhou City & Jiande City & 29.341 & 119.067 & Anthracite & Small & Dong shan \\
\hline 30 & Zhejiang Province & Jinhua City & Yiwu City & 29.377 & 120.023 & Anthracite & Small & Wu zao \\
\hline 31 & Zhejiang Province & Taizhou City & Tiantai City & 29.114 & 120.847 & Lignite & Small & Zi ning \\
\hline 33 & Zhejiang Province & Quzhou City & Kaihua County & 28.998 & 118.413 & Bituminous & Small & Ye jia tang \\
\hline 34 & Zhejiang Province & Quzhou City & Changshan County & 28.876 & 118.320 & Bituminous & Small & Qiu chuan \\
\hline 36 & Zhejiang Province & Quzhou City & Jiangshan City & 28.623 & 118.547 & Bituminous & Small & Dao tang shan \\
\hline 37 & Zhejiang Province & Quzhou City & Jiangshan City & 28.586 & 118.476 & Bituminous & Small & Zheng tang \\
\hline 38 & Zhejiang Province & Lishui City & Songyang County & 28.376 & 119.637 & Anthracite & Small & Mao nong \\
\hline
\end{tabular}

